\title{
Science-Driven Candidate Search for New Scintillator Materials FY 2014 Annual Report
}

SN Kerisit

F Gao

YL Xie
LW Campbell

D Wu

MP Prange

October 2014

Pacific Northwest

NATIONAL LABORATORY

Proudly Operated by Battelle Since 1965 


\title{
DISCLAIMER
}

This report was prepared as an account of work sponsored by an agency of the United States Government. Neither the United States Government nor any agency thereof, nor Battelle Memorial Institute, nor any of their employees, makes any warranty, express or implied, or assumes any legal liability or responsibility for the accuracy, completeness, or usefulness of any information, apparatus, product, or process disclosed, or represents that its use would not infringe privately owned rights. Reference herein to any specific commercial product, process, or service by trade name, trademark, manufacturer, or otherwise does not necessarily constitute or imply its endorsement, recommendation, or favoring by the United States Government or any agency thereof, or Battelle Memorial Institute. The views and opinions of authors expressed herein do not necessarily state or reflect those of the United States Government or any agency thereof.

\author{
PACIFIC NORTHWEST NATIONAL LABORATORY \\ operated by \\ BATTELLE \\ for the \\ UNITED STATES DEPARTMENT OF ENERGY \\ under Contract DE-AC05-76RL01830
}

Printed in the United States of America

Available to DOE and DOE contractors from the

Office of Scientific and Technical Information,

P.O. Box 62, Oak Ridge, TN 37831-0062;

ph: (865) 576-8401

fax: $(865) 576-5728$

email: reports@adonis.osti.gov

Available to the public from the National Technical Information Service

5301 Shawnee Rd., Alexandria, VA 22312

ph: (800) 553-NTIS (6847)

email: orders $a$ ntis.gov <http://www.ntis.gov/about/form.aspx>

Online ordering: http://www.ntis.gov

This document was printed on recycled paper. 


\section{Science-Driven Candidate Search for New Scintillator Materials FY 2014 Annual Report}

$\begin{array}{ll}\text { SN Kerisit } & \text { LW Campbell } \\ \text { F Gao } & \text { D Wu } \\ \text { YL Xie } & \text { MP Prange }\end{array}$

October 2014

Prepared for

the U.S. Department of Energy

under Contract DE-AC05-76RL01830

Pacific Northwest National Laboratory

Richland, Washington 99352 


\begin{abstract}
This annual reports presents work carried out during Fiscal Year (FY) 2014 at Pacific Northwest National Laboratory (PNNL) under the project entitled "Science-Driven Candidate Search for New Scintillator Materials” (Project number: PL13-SciDriScintMat-PD05) and led by Drs. Fei Gao and Sebastien N. Kerisit.
\end{abstract}

This project is divided into three tasks:

1) Ab initio calculations of electronic properties, electronic response functions and secondary particle spectra;

2) Intrinsic response properties, theoretical light yield, and microscopic description of ionization tracks; and

3) Kinetics and efficiency of scintillation: nonproportionality, intrinsic energy resolution, and pulse shape discrimination.

Detailed information on the results obtained in each of the three tasks is provided in this Annual Report. Furthermore, peer-reviewed articles published this FY or currently under review and presentations given this FY are included in Appendix.

This work was supported by the National Nuclear Security Administration, Office of Nuclear Nonproliferation Research and Development (DNN R\&D/NA-22), of the U.S. Department of Energy (DOE). 


\section{Acronyms and Abbreviations}

$\begin{array}{ll}\text { ABINIT } & \text { Electronic structure computer package } \\ \text { CBM } & \text { Conduction Band Minimum } \\ \text { DOE } & \text { Department of Energy } \\ \text { DOS } & \text { Density of States } \\ \text { ERSP } & \text { Electronic ReSPonse } \\ \text { FY } & \text { Fiscal Year } \\ \text { GGA } & \text { Generalized Gradient Approximation } \\ \text { GGA+U } & \text { GGA with Hubbard correction } \\ \text { KMC } & \text { Kinetic Monte Carlo } \\ \text { LCPM } & \text { Local Principal Curve Method } \\ \text { LDA } & \text { Local-Density Approximation } \\ \text { LLNL } & \text { Lawrence Livermore National Laboratory } \\ \text { LO } & \text { Longitudinal Optical } \\ \text { NWEGRIM } & \text { NorthWest Electron and Gamma-Ray Interaction with Matter } \\ \text { PNNL } & \text { Pacific Northwest National Laboratory } \\ \text { STE } & \text { Self-Trapped Exciton } \\ \text { STEL } & \text { Self-Trapped Electron } \\ \text { STH } & \text { Self-Trapped Hole } \\ \text { VBM } & \text { Valence Band Maximum } \\ \text { WFU } & \text { Wake Forest University } \\ \text { YAG } & \left.\text { Yttrium Aluminum Garnet (Y }{ }_{3} \mathrm{Al}_{5} \mathrm{O}_{12}\right) \\ \text { YAP } & \text { Yttrium Aluminum Perovskite }\left(\mathrm{YAlO}_{3}\right) \\ & \end{array}$




\section{Contents}

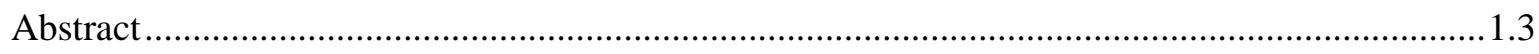

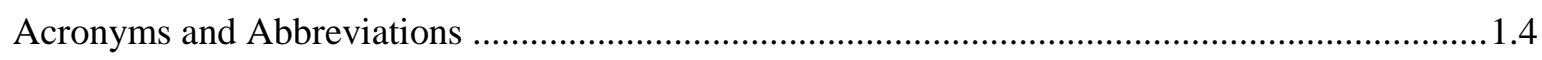

1.0 Objective, Strategy and Findings Summary .................................................................. 1.9

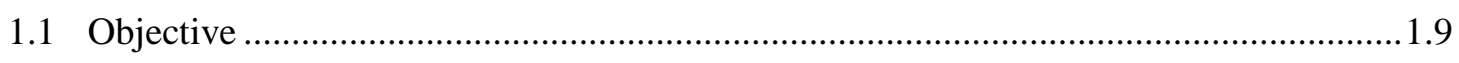

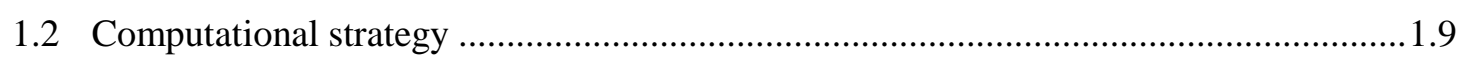

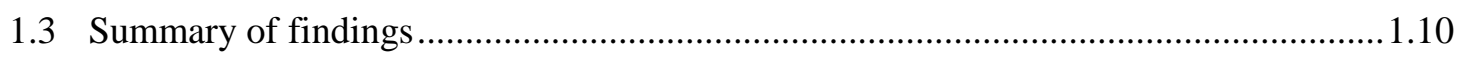

2.0 Task 1: Ab initio calculations of electronic properties, electronic response functions and

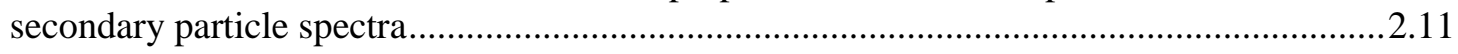

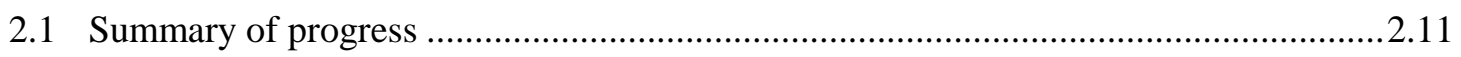

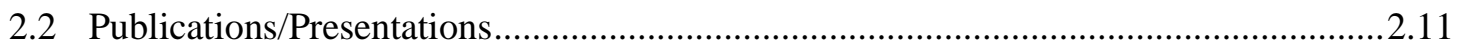

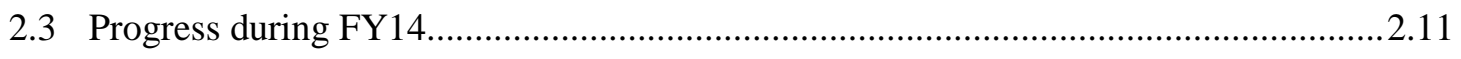

2.3.1 New theoretical approaches for more realistic thermalization models...............2.11

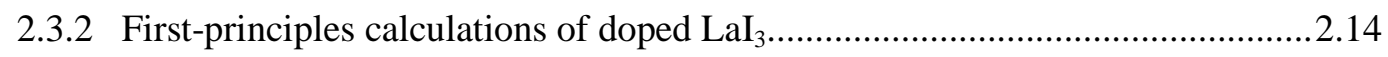

2.3.3 Electronic response to heavy particles ...........................................................2.15

3.0 Task 2: Intrinsic response properties, theoretical light yield, and microscopic description of

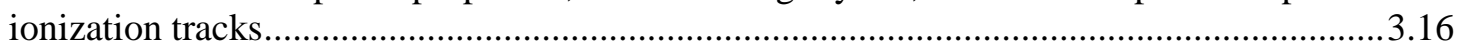

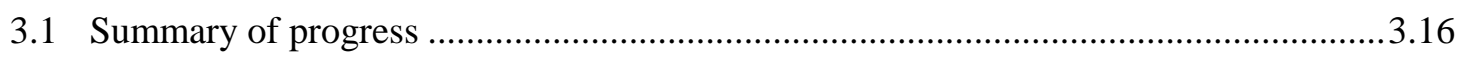

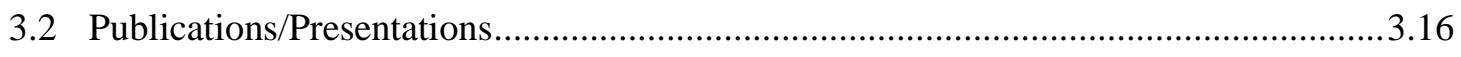

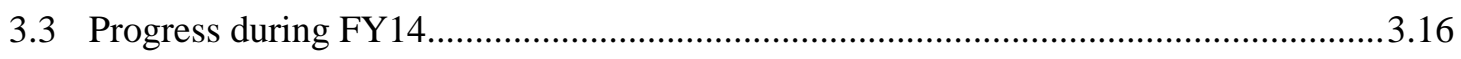

3.3.1 Lanthanum halide series ................................................................................ 3.17

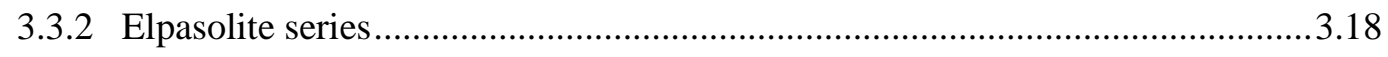

3.3.3 Local principal curve analysis of ionization tracks ............................................19

4.0 Task 3: Kinetics and efficiency of scintillation: nonproportionality, intrinsic energy resolution, and pulse shape discrimination ..........................................................................2.20

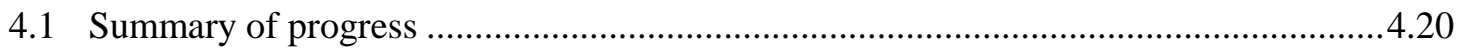

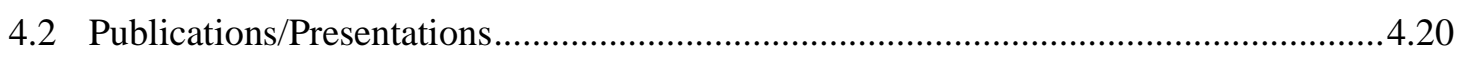

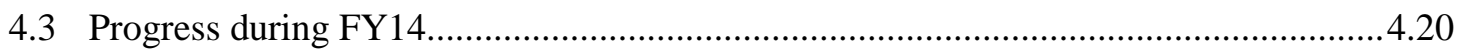

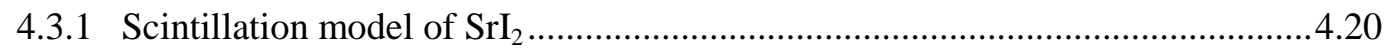

4.3.2 Electron and hole thermalization in alkali halides ............................................4.23 


\section{Figures}

Fig. 1. Flow chart of computational modeling process (with names of the codes developed in this project shown in the upper left-hand corners of each box).

Fig. 2. Computed phonon band structure of CsI........................................................................12

Fig. 3. Ab initio calculations of the rate of energy transferred to the CsI lattice by emission and absorption of phonons at $0 \mathrm{~K}$ and at room temperature. Also shown is the room temperature

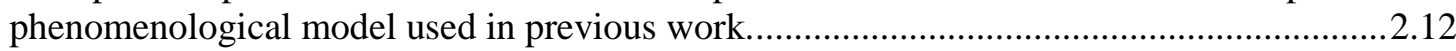

Fig. 4. Computed band structure and density of states in CsI compared to the effective mass approximation.

Fig. 5. Average of the magnitude of the group velocity calculated from the band structure using Equation 2. Also shown are the velocities computed from the effective mass approximation and a model applicable to the KMC program.

Fig. 6. Density of states of pure $\mathrm{LaI}_{3}$ crystal from GGA, GGA+U, and GW calculations.

Fig. 7. Density of states of $\mathrm{LaI}_{3}$ pure and doped with $\mathrm{Ce}^{3+}$ and $\mathrm{Bi}^{3+}$ in the ground state, as calculated with GGA+U.

Fig. 8. Variation of the mean energy per electron-hole as a function of incident photon energy..3.17

Fig. 9. Theoretical maximum light yields of a range of scintillators together with experimental values reported in the literature.

Fig. 10. Calculated spatial distributions of electron-hole pairs for a $10-\mathrm{keV}$ photon event in CLLB and CLLC. Holes are shown in yellow and electrons generated via interband transition, plasmon, ionization event, and relaxation are shown in green, red, blue, and purple, respectively.

Fig. 11. Simulated spatial distributions of electron-hole pairs with local principal curves for two ionization tracks produced by $10-\mathrm{keV}$ photon events in CsI.

Fig. 12. Comparison of calculated and experimental percentages of the impurity emission in Eudoped $\mathrm{SrI}_{2}$. Also shown is the impurity concentration required to optimize agreement with experimental data.

Fig. 13. Temperature dependence of the total light yield, light yield from $\left(\mathrm{Eu}^{2+}\right)^{*}$ emission, and light yield from defect emission, from experiment and KMC simulations.....

Fig. 14. Experimental and simulated scintillation decay curves of a $6-\mathrm{mm}$ thick $\mathrm{SrI}_{2}: 2 \% \mathrm{Eu}$ crystals at 295, 450 and $600 \mathrm{~K}$.

Fig. 15. Experimental and simulated decay curves of 2-mm, 6-mm, and $15-\mathrm{mm}$ thick $\mathrm{SrI}_{2}: 2 \% \mathrm{Eu}^{2+}$ crystals at $295 \mathrm{~K}$.

Fig. 16. Thermalization time distributions of electrons with kinetic energies of 0.1 or $3.0 \mathrm{~V}$ as obtained with the effective mass approximation (EMA) and group velocity (GV) models. .4.24

Fig. 17. Distance distributions of recombined electrons following irradiation of CsI with 2-keV $\gamma$ rays for three different initial kinetic energies of the holes $\left(E_{h}\right)$.

Fig. 18. Distance distributions of stopped electrons (left) and holes (right) following irradiation of CsI with 2-keV $\gamma$-rays for three different initial kinetic energies of the holes $\left(E_{h}\right)$. Also shown is the distance distribution obtained in previous work using an electron band mass of $1 m_{0}$ with polaron effects and $\mathrm{E}_{\mathrm{h}}=0 \mathrm{eV}$.

Fig. 19. Time distributions of stopped electrons (left) and holes (right) following irradiation of CsI with 2-keV $\gamma$-rays for three different initial kinetic energies of the holes $\left(E_{h}\right)$. Also 
shown is the distance distribution obtained in previous work using an electron band mass of $1 m_{0}$ with polaron effects and $\mathrm{E}_{\mathrm{h}}=0 \mathrm{eV}$.

Fig. 20. Distance distributions of stopped electrons and holes following irradiation of NaI with 2-

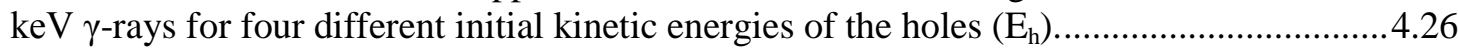

Fig. 21. Time distributions of stopped electrons and holes following irradiation of $\mathrm{NaI}$ with 2-keV $\gamma$-rays for four different initial kinetic energies of the holes $\left(E_{h}\right)$. .4 .26 


\section{Tables}

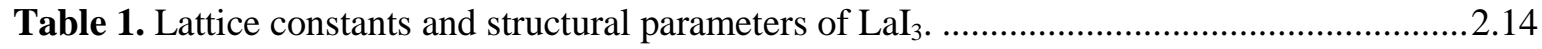

Table 2. Intrinsic properties of scintillators in the lanthanum halide series, as calculated by

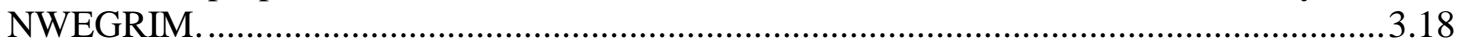

Table 3. Intrinsic properties of scintillators in the elpasolite series, as calculated by NWEGRIM.3.18 


\subsection{Objective, Strategy and Findings Summary}

\subsection{Objective}

This project aims to develop, apply, and validate a suite of computational modeling tools to explore the relationship between materials properties and scintillator performance by determining the key elementary processes that give rise to a material's light yield, decay times, nonproportionality, energy resolution, and potential particle discrimination ability. These state-of-the-art capabilities will yield a predictive simulation framework for evaluating candidate materials and improve the performance of existing scintillators.

This objective will be met, in part, by on-going collaborations with researchers at Lawrence Livermore National Laboratory, who are generating first-principles inputs for our kinetic model of scintillation, and researchers at Wake Forest University, who are conducting experiments to validate model predictions.

\subsection{Computational strategy}

We have developed and applied a suite of physics models at multiple scales to simulate the three main stages of the scintillation process in $\gamma$-ray detectors: initial energy cascade to produce energetic particles; thermalization of particles into low-energy excitations, and relaxation of excitations to produce scintillation light. Simulations of these individual stages are combined to produce a unified approach that can predict scintillator performance from knowledge of a few material parameters (Fig. 1).

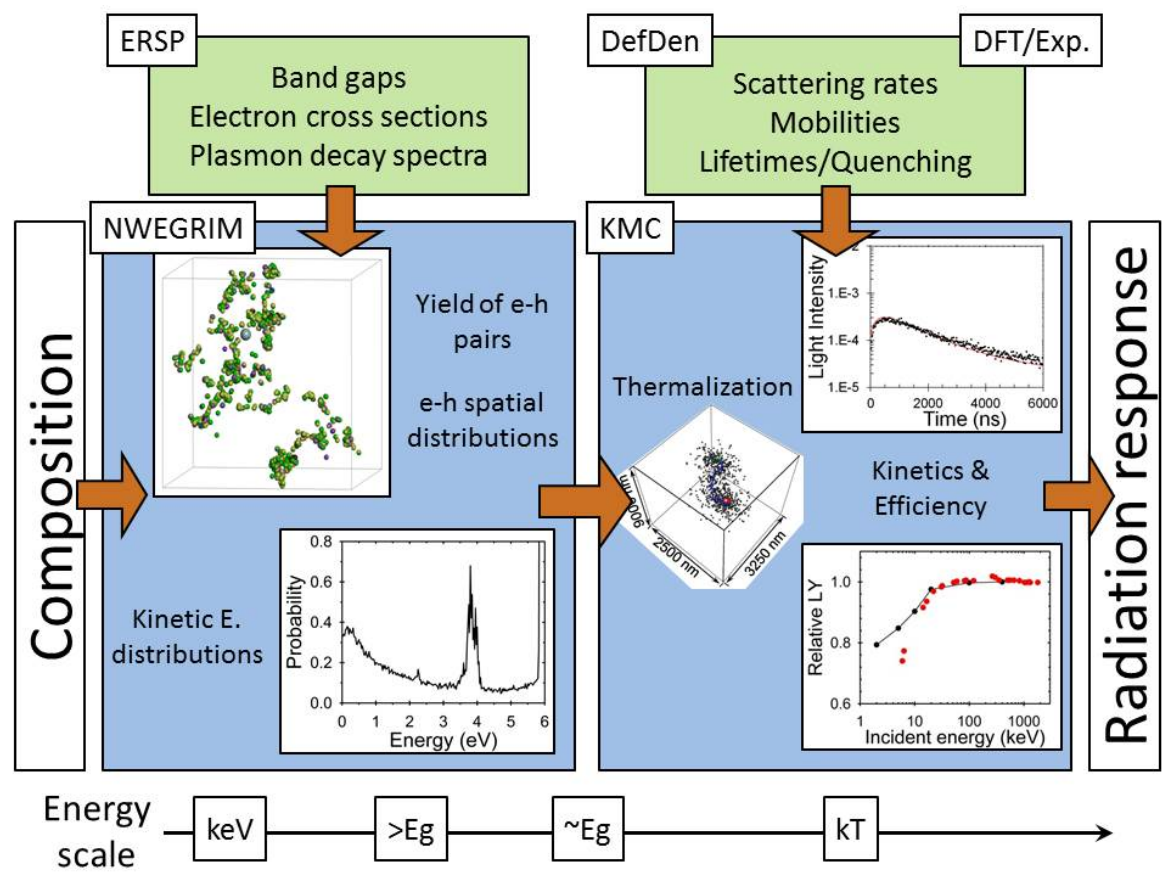

Fig. 1. Flow chart of computational modeling process (with names of the codes developed in this project shown in the upper lefthand corners of each box). 


\subsection{Summary of findings}

This FY, we continued to make progress in developing and applying a suite of simulation tools for modeling all three stages of scintillation. In addition, these simulation tools were used to begin to make predictions of potential bright scintillators and identify current scintillators with the greatest potential for improvement. Collaborations with NA-22-funded researchers at LLNL and WFU also continued this FY to generate first-principles inputs for our kinetic model of scintillation (LLNL) and to parameterize our scintillation models and validate our model predictions (WFU).

In Task 1, new theoretical capabilities were developed to provide first-principles based input to the simulations of the three scintillation stages. In particular, we pursued major improvements of the current models of thermalization. Previous theoretical predictions of the distances traveled by energetic electrons during thermalization have highlighted the importance of the extent of charge separation in scintillators. Therefore, an accurate, quantitative determination of the spatial and temporal scales of thermalization is critical to the development of predictive models of nonproportionality. Consequently, we developed a quantum-mechanical model for calculating the energy relaxation rates of energetic particles (DefDen) that will allow for more realistic and reliable predictions. In addition, we developed an improved treatment of particle dispersion to overcome the limitations of the effective mass approximation. Application to the calculation of electron and hole thermalization in CsI and NaI was completed in Task 3.

In Task 2, simulations of the yield and spatial distribution of electron-hole pairs resulting from $\gamma$-ray irradiation were performed for two material series: the lanthanum halide series and the elpasolite series. The simulations predicted $\mathrm{LaI}_{3}$ to be a potential very bright scintillator with a theoretical maximum light yield significantly higher than that of bright and widely-studied $\mathrm{LaBr}_{3}$. The only measured light yield reported in the literature for (Ce-doped) $\mathrm{LaI}_{3}$ is disappointedly small ( 1/10 of theoretical maximum), most likely due to the close proximity of the Ce energy levels to the host conduction band, which allows for extensive electron thermal release. Therefore, $a b$ initio calculations were initiated this FY in Task 1 to identify suitable dopants for this promising material, whereby both lanthanide $\left(e . g . \mathrm{Pr}^{3+}, \mathrm{Nd}^{3+}\right.$, and $\mathrm{Gd}^{3+}$ ) and $6 s^{2}\left(\mathrm{Tl}^{+}, \mathrm{Pb}^{2+}\right.$, and $\left.\mathrm{Bi}^{3+}\right)$ ions are being considered. The calculations suggest that the presence of $\mathrm{Bi} 6 p$ levels in the band gap of $\mathrm{LaI}_{3}$ could enable scintillation via relaxation of $\mathrm{V}_{\mathrm{k}}-\mathrm{Bi}^{2+}$ pairs, pointing to $\mathrm{Bi}$ as a potential suitable dopant.

In Task 3, we continued our developments of kinetic Monte Carlo (KMC) models of scintillation, completing the work on $\mathrm{NaI}$ and extending our efforts to $\mathrm{SrI}_{2}$. In particular, an important step in building a full predictive model of nonproportionality is to compare outputs of some the component simulations to intermediate experimental data measured under simpler experimental circumstances than a high-energy $\gamma$ ray ionization track. Consequently, in collaboration with WFU, our KMC model of scintillation in NaI was compared against several types of experimental data including the luminescence yield as a function of excitation density following ultraviolet excitation. This activity allowed for the parameterization of processes that describe the spatial extent of exciton annihilation. Incorporation of parameterized interactions between excitations will enable more realistic simulations of nonproportionality. A similar approach is being applied to study $\mathrm{SrI}_{2}$.

A task-by-task description of this FY's findings follows. All publications and invited presentations are attached in Appendix A in order of presentation. Also attached is a briefing given to HQ Program Manager Dr. David Beach on August 262014 in Washington, DC. 


\subsection{Task 1: $A b$ initio calculations of electronic properties, electronic response functions and secondary particle spectra}

\subsection{Summary of progress}

Two significant improvements of the current models of hot particle thermalization were achieved this FY: 1) a quantum-mechanical model of energy relaxation rates of hot particles was completed and applied to CsI; and 2) a more realistic model of hot particle dispersion was developed and applied to alkali halide scintillators. Additionally, the electronic structures of pure, Ce-doped, and Bi-doped $\mathrm{LaI}_{3}$ were determined based on several first-principles methods. Finally, our electronic response code (ERSP) was modified to treat the electronic response to heavy radiation particles.

\subsection{Publications/Presentations}

R.M. Van Ginhoven and P.A. Schultz Journal of Physics: Condensed Matter 25 (2013) 495504

M.P. Prange, L.W. Campbell, and F. Gao Physical Review B to be submitted in October 2014.

\subsection{Progress during FY14}

Three activities were pursued under this task in FY14, namely the development of new theoretical approaches for more realistic thermalization models, the application of first-principles calculations for identifying suitable dopants for $\mathrm{LaI}_{3}$, and the implementation of the response to heavy particles in ERSP.

\subsubsection{New theoretical approaches for more realistic thermalization models}

Previous theoretical predictions of the distances traveled by hot electrons during thermalization have highlighted the importance of the extent of charge separation in scintillators. Electrons and holes persist as free particles during thermalization in materials such as CsI and $\mathrm{NaI}$ due to their low-energy phonons and thus slow cooling. In turn, this contributes to nonproportionality through third-order nonlinear quenching in regions with high electron-hole pair densities and extensive charge separation in low-density regions. Therefore, an accurate, quantitative determination of the thermalization distances and time scale of thermalization is critical to the development of predictive models of nonproportionality. Consequently, this FY, we pursued two major improvements of the current models of thermalization that are allowing for more realistic and reliable predictions: (1) we developed a quantum-mechanical model for calculating the energy relaxation rates of hot particles; and (2) we developed an improved treatment of particle dispersion to model thermalization and applied it to alkali halide scintillators.

Quantum-mechanical model of energy relaxation rates of fast particles. Our treatment of hot particle scattering is based on ABINIT calculations of the response of electron density to various displacements of the atoms in the unit cell, from which scattering rates can be calculated using our newly developed code. In our model, fast particles are represented as classical particles traveling with a specified velocity. Both absorption and emission of optical and acoustic phonons can be calculated using this approach. 

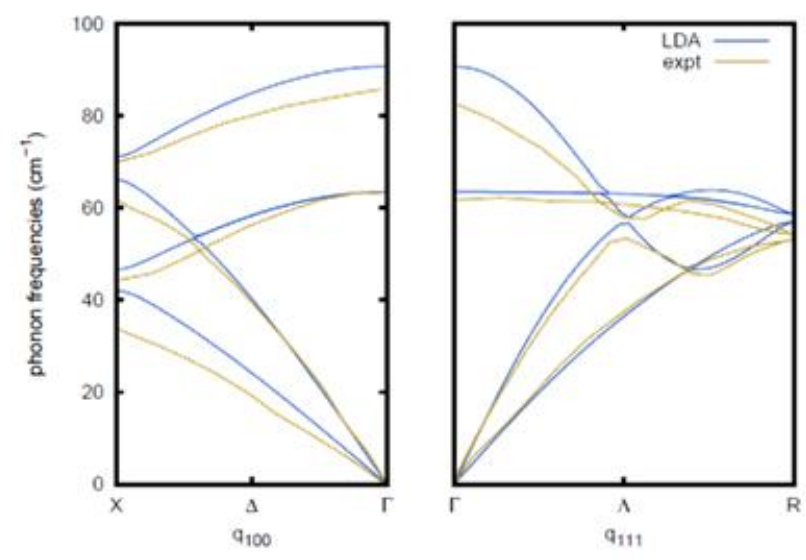

Fig. 2. Computed phonon band structure of CsI.

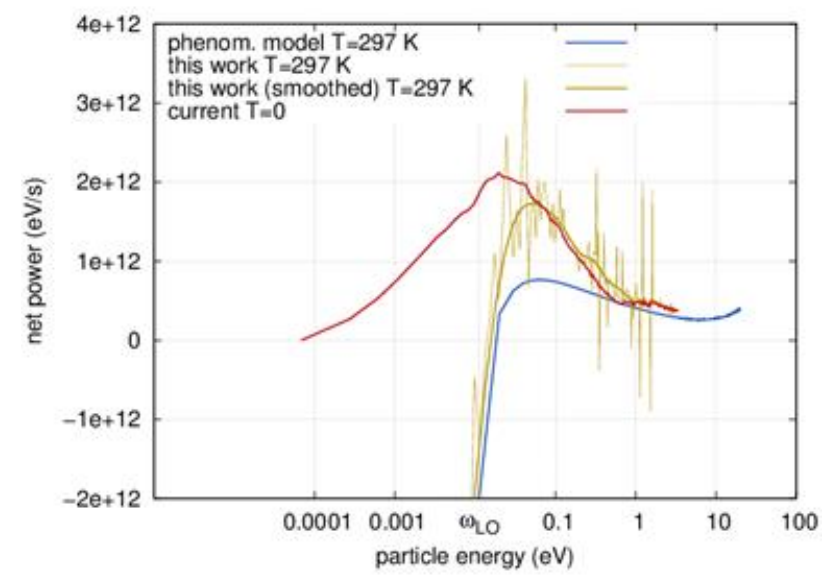

Fig. 3. Ab initio calculations of the rate of energy transferred to the CsI lattice by emission and absorption of phonons at $0 \mathrm{~K}$ and at room temperature. Also shown is the room temperature phenomenological model used in previous work.

CsI has been used throughout this code development stage as a model system. We first verified that our approach yielded satisfactory agreement with the experimental phonon band structure of CsI (Fig. 1). The $a b$ initio calculated rates of energy relaxation in CsI are shown in Fig. 2. Good agreement between our $a b$ initio calculations and the phenomenological model for the Frölich-type scattering of polar phonon modes is observed. In contrast, the phenomenological model for acoustic phonon modes fails to reproduce the results of our $a b$ initio calculations. The treatment of acoustic modes in the phenomenological model is based on the deformation potential approximation. The derivation of the deformation potential approximation uses the assumption that the scattering occurs only near the origin of momentum-transfer space and that the electrons that are scattered are confined to a small region of the Brillouin zone. These assumptions are not satisfied during thermalization following the energy cascade of scintillation. The deformation potential approximation predicts that electrons will not be slowed significantly by acoustic modes if they have kinetic energies below $\sim 1 \mathrm{eV}$. In contrast, we find that the acoustic modes in CsI do contribute to slowing of charged particles at low energy. This can be seen in Fig. 2, which shows significantly more transferred power at particle energies between the LO phonon frequency and $0.5 \mathrm{eV}$.

The calculated net transferred power shows fluctuations around a smooth background. These fluctuations arise from two sources: 1) cancelation between stimulated phonon emission and absorption terms, which become large when the phonon modes have significant thermal occupation; and 2) dependence on the direction of the particle velocity. The first source is numerical error and, in principle, could be eliminated with fine enough grids and small enough tolerances. The second source is inherently due to the anisotropy of the material.

A significant advantage of this approach is that materials with complicated unit cells, and hence complicated phonon excitations, to which it is difficult to apply phenomenological models, can be treated.

Improved model of particle dispersion. In previous thermalization calculations, electrons have been assumed to move according to the effective mass dispersion relation 


$$
E=\frac{1}{2} m_{b} v^{2}
$$

which relates the kinetic energy, $E$, of an electron to its speed, $v$, through its effective band mass, $m_{b}$. The effective mass is related to the curvature of the band structure and gives a good description of electrons near the CBM and holes near the VBM, i.e. for particles with kinetic energy on the order of a fraction of one electronvolt. However, a majority of the hot electrons generated during the energy cascade have higher energies and are thus found in a variety of energy bands.

Therefore, to better model the dynamics of highly energetics particles, we have developed a model based on band structure calculations that can be used for both electrons and holes and is applicable at arbitrary energies. The model relates the speed with which a particle moves to its energy via the equation:

$$
v(E)=\frac{\sum_{n} \int_{B Z} d^{3} k\left|\frac{d}{d \vec{k}} E_{n}(\vec{k})\right| \delta\left(E-E_{n}(\vec{k})\right)}{\sum_{n} \int_{B Z} d^{3} k \delta\left(E-E_{n}(\vec{k})\right)}
$$

which averages the wave-mechanical group velocity over surfaces of constant energy. This expression reduces to the effective mass approximation near the band extrema for systems with isotropic effective mass and includes contributions from all possible quasi-particle states of energy $E$.

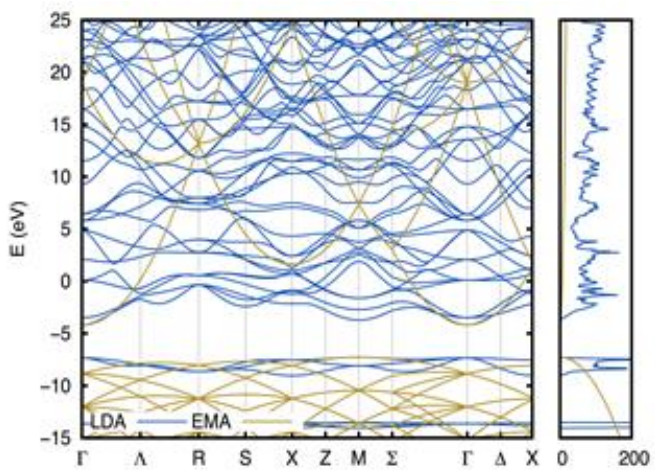

Fig. 4. Computed band structure and density of states in CsI compared to the effective mass approximation.

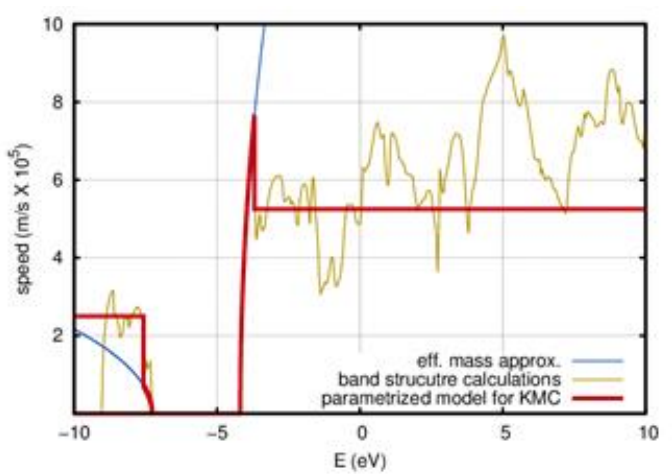

Fig. 5. Average of the magnitude of the group velocity calculated from the band structure using Equation 2. Also shown are the velocities computed from the effective mass approximation and a model applicable to the KMC program.

Fig. 3 shows the computed energy bands in CsI together with the results of the effective mass approximation based on the curvature of the band structure near minima for electrons and maxima for holes. Fig. 3 demonstrates that the agreement between the effective mass approximation and the band structure is good only near the CBM for electrons, which is the $\Gamma$-point in the plotted case, and the VBM for holes, which occurs at $\mathrm{M}$ in CsI. The band masses thus obtained were $0.31 \mathrm{~m}_{0}$ and $2.27 \mathrm{~m}_{0}$ for holes and electrons, respectively. The calculated band masses are in good agreement with those calculated by Setyawan et al. [IEEE Trans. Nucl. Sci. 56 (2009) 2989].

Fig. 4 shows the average velocities of holes and electrons calculated from the band structure using Equation 2 together with those obtained based on the effective mass approximation and Equation 1. The 
effective mass approximation gives a good description of quasi-particle velocities near the edges of the band gap; however, it considerably overestimates the velocities of quasi-particles at higher energies with respect to the computed group velocities given by Equation 2. The results of the average of the group velocities were used to parameterize the KMC model of thermalization and the results of these simulations are presented in Task 3.

\subsubsection{First-principles calculations of doped $\mathrm{Lal}_{3}$}

First-principles calculations of doping effects on the electronic structure of $\mathrm{LaI}_{3}$ were performed this FY to identify suitable dopants for this scintillator material. $\mathrm{LaI}_{3}$ has been identified in our previous modeling work has a potentially very bright scintillator; however, current experimental light yields of Cedoped $\mathrm{LaI}_{3}$ are much lower than expected due to the close proximity of the Ce energy levels to the CBM. Therefore, identifying a doping scheme that minimizes electron thermal release is critical to fully exploit $\mathrm{LaI}_{3}$ predicted performance.

First, we calculated the structural and electronic properties of pure $\mathrm{LaI}_{3}$ crystal using different electronic structure methods (GGA, GGA+U, GW). $\mathrm{LaI}_{3}$ adopts an orthorhombic lattice with the $\mathrm{PuBr}_{3}$ structure (space group $\mathrm{cmcm}$ ). There are 16 atoms in the unit cell, with four La atoms in positions 4(c), $\pm(0, u, 1 / 4)$, four I atoms in position 4 (c), $\pm(0, v, 1 / 4)$, and eight I atoms in positions $8(f), \pm(0, m, n ; 0, m, 1 / 2-$ $n)$. The lattice constants $(a, b, c)$ and the structural parameters $(u, v, m, n)$ obtained from our GGA and $\mathrm{GGA}+\mathrm{U}\left(\mathrm{U}_{\text {eff }}(\mathrm{La})=10.32 \mathrm{eV}\right)$ calculations are listed with available experimental data in Table 1 . As the GGA method usually underestimates the binding energy, the lattice constants from our calculations are slightly larger than the experimental results. Nevertheless, the calculated crystal structure is in good agreement with experimental data.

Table 1. Lattice constants and structural parameters of $\mathrm{LaI}_{3}$.

\begin{tabular}{cccc}
\hline Property & GGA & GGA+U & Experiment $^{1,2}$ \\
\hline$a(\AA)$ & 4.446 & 4.469 & 4.37 \\
$b(\AA)$ & 15.735 & 15.684 & 14.01 \\
$c(\AA)$ & 10.158 & 10.195 & 10.04 \\
$u$ & 0.2591 & 0.2594 & 0.2562 \\
$v$ & -0.0952 & -0.0925 & -0.0789 \\
$m$ & 0.3440 & 0.3452 & 0.3557 \\
$n$ & -0.0670 & -0.0666 & -0.0661 \\
\hline
\end{tabular}

${ }^{1}$ Asprey et al. Inorg. Chem. 3 (1964) 1137

${ }^{2}$ Levy et al. Acta Cryst. B 31 (1975) 880

Fig. 5 shows DOS for $\mathrm{LaI}_{3}$ using GGA, GGA+U, and GW methods. As expected, the GGA calculations underestimate the band gap significantly (2.1 eV vs. $3.3 \mathrm{eV}$ (Bessiere et al. Nucl. Instr. Meth. A 537 (2005) 22) and $3.9 \mathrm{eV}$ (Jungmann et al. Z. Phys. B 97 (1995) 25) from experiments). In contrast, $\mathrm{GW}$ predicts a band gap of $3.8 \mathrm{eV}$ in good agreement with the range of published experimental values. Although the use of the GGA+U method does not improve the band gap prediction over that predicted with GGA, it produces the correct ordering of La $5 d$ and $4 f$ states determined by GW, while GGA does not. 
For the dopant calculations, we used a supercell containing 128 atoms constructed from the optimized unit cell with one La atom replaced by the dopant element. The dopants under consideration are lanthanide ions $\mathrm{Ce}^{3+}, \mathrm{Pr}^{3+}, \mathrm{Nd}^{3+}, \mathrm{Eu}^{3+}$, and $\mathrm{Gd}^{3+}$ and $6 s^{2}$ ions $\mathrm{Tl}^{+}, \mathrm{Pb}^{2+}$, and $\mathrm{Bi}^{3+}$. Ce-doped $\mathrm{LaI}_{3}$ was first calculated as a reference as $\mathrm{Ce}^{3+}$ was previously evaluated as a potential dopant experimentally and in theoretical work by Canning et al. [Phys. Rev. B 83 (2011) 125115]. Fig. 6 shows the DOS of Ce-doped $\mathrm{LaI}_{3}$ from our ground-state GGA+U calculation $\left(\mathrm{U}_{\text {eff }}(\mathrm{Ce})=2.2 \mathrm{eV}\right)$. We can see a localized $\mathrm{Ce}^{3+} 4 f$ state at the VBM. This level is positioned approximately $0.2 \mathrm{eV}$ below that obtained in the calculations of Canning et al. and expected based on the experiments of Bessiere et al. This could be resolved by slightly modifying the value of $U_{\text {eff }}(\mathrm{Ce})$, which we are currently investigating. Once the optimal value of $U_{\text {eff }}$ on the lanthanide dopant is identified, it will be applied to the other candidate lanthanide dopants.

Fig. 6 also shows the DOS of Bi-doped $\mathrm{LaI}_{3}$ as obtained using GGA+U. The $\mathrm{Bi} 6 p$ level is located slightly below the CBM. Unlike for the lanthanide ions, which exhibit topmost occupied states slightly above the VBM, $6 s^{2}$ ions, such as $\mathrm{Bi}^{3+}$, show topmost occupied states below the valence band. However, their $6 p$ states can be positioned in the host band gap and, therefore, can be attractive to electrons. As a result, $\mathrm{Bi}^{2+}$ could attract holes in the valence band $\left(\mathrm{V}_{\mathrm{k}}\right.$ centers) and potentially form Bi-bound excitons. This potential alternative scintillation mechanism, recently put forward by Du [J. Mater. Chem. C 2 (2014) 4784], could eliminate the need to have two activator states in the host band gap and, therefore, provide a way to enhance the scintillation yield of small gap scintillators such as $\mathrm{LaI}_{3}$.

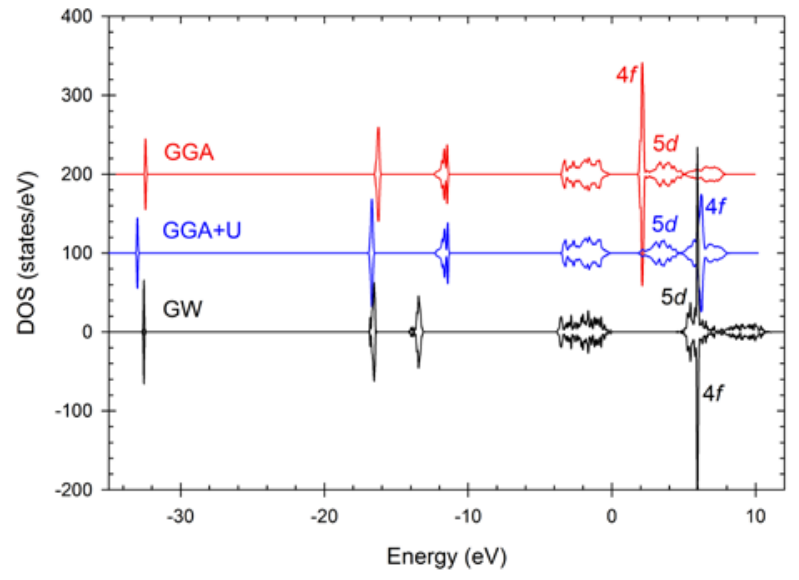

Fig. 6. Density of states of pure $\mathrm{LaI}_{3}$ crystal from GGA, $\mathrm{GGA}+\mathrm{U}$, and GW calculations.

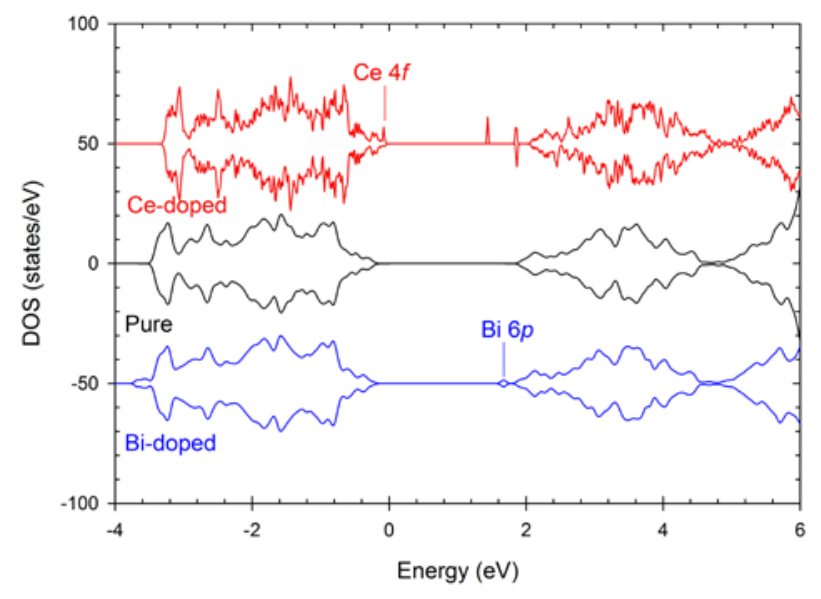

Fig. 7. Density of states of $\mathrm{LaI}_{3}$ pure and doped with $\mathrm{Ce}^{3+}$ and $\mathrm{Bi}^{3+}$ in the ground state, as calculated with GGA+U.

\subsubsection{Electronic response to heavy particles}

The electronic response code ERSP has been modified to calculate the low momentum electronic scattering and energy transfer for heavy radiation particles, such as alpha particles, protons, and ions. While this momentum grid is adequate for electrons and contains the dominant part of losses of high energy heavy particles, at low energies the kinematically allowed excitations of heavy particles are outside of the scattering momenta calculated. Modifications of ERSP to include higher momentum transfers are currently being pursued. When finished, it is expected that this will allow ab initio calculations of electronic cross sections and stopping powers of alpha particles and ions from neutron recoils and neutron nuclear interactions. 


\title{
3.0 Task 2: Intrinsic response properties, theoretical light yield, and microscopic description of ionization tracks
}

\subsection{Summary of progress}

Cross sections were derived and NWEGRIM simulations were performed for two series of scintillator materials, namely the lanthanum halide series and the elpasolite series. $\mathrm{LaI}_{3}$ was identified as a potentially very bright scintillator. Theoretical maximum light yields of all scintillators simulated to date were compared to experimental data to determine materials with the greatest potential for improvement. A local principal curve analysis of ionization tracks was employed to begin to relate track structures to material performance.

\subsection{Publications/Presentations}

\author{
R.T. Williams, J.Q. Grim, Q. Li, K.B. Ucer, G.A. Bizarri, S. Kerisit, F. Gao, P. Bhattacharya, E. \\ Tupitsyn, E. Rowe, V.M. Buliga, and A. Burger Proceedings of SPIE 8852 (2013) 88520J \\ F. Gao, Y.L. Xie, Z.G. Wang, S. Kerisit, D.X. Wu, L.W. Campbell, R.M. Van Ginhoven, and M. \\ Prange Journal of Applied Physics 114 (2013) 173512 \\ (Invited) F. Gao Nuclear Weapons and Material Security Team Program Review, May 21 2014, \\ Argonne National Laboratory, Argonne, Illinois
}

\subsection{Progress during FY14}

Building upon our experience simulating common scintillators such as CsI, $\mathrm{NaI}, \mathrm{SrI}_{2}$, and YAG, we simulated materials in series this FY in an effort to search for new scintillator materials with improved scintillation performance. Our purpose is twofold: firstly, we aim to identify trends and find the best candidates within a given scintillator series; and, secondly, by studying materials with common constituent elements, we are looking to find possible correlations between scintillation performance and intrinsic properties, which, in turn, can be used as a guide for the search for new materials. This FY, we simulated two scintillator series, namely the lanthanum halide series $\left(\mathrm{LaF}_{3}, \mathrm{LaCl}_{3}, \mathrm{LaBr}_{3}\right.$, and $\left.\mathrm{LaI}_{3}\right)$ and the elpasolite series $\left(\mathrm{Cs}_{2} \mathrm{LiLaBr}_{6}\right.$ (CLLB), $\mathrm{Cs}_{2} \mathrm{LiLaCl}_{6}$ (CLLC), $\mathrm{Cs}_{2} \mathrm{LiYBr}_{6}(\mathrm{CLYB})$, and $\mathrm{Cs}_{2} \mathrm{LiYCl}_{6}$ (CLYC)).

For both series, we first determined various cross sections for electrons, including interband transition, plasmon excitation, and inner-shell ionization. Using these cross sections in NWEGRIM, energy cascade simulations were carried out for incident photon energies ranging from $50 \mathrm{eV}$ to $662 \mathrm{keV}$. To obtain good statistics, we simulated $10^{5}$ photon events for each material. The primary and secondary electrons were followed until their energies were less than the cut-off energy, which was set at twice the band gap energy of the material. The distributions of the number of produced electrons were used to determine the mean energy per electron-hole pair, $W$, the Fano factor (variance of the number of electronhole pairs produced), $F$, and the theoretical maximum light yield. Other intrinsic properties also obtained from these simulations were the electron-hole pair generation mechanisms and the spatial structure of ionization tracks. 


\subsubsection{Lanthanum halide series}

Similarly to what was observed for other scintillator materials, $W$ fluctuates at low energies but approaches a constant value at higher energies for the lanthanum halide series, as shown in Fig. 7 for $\mathrm{LaI}_{3}$ for example. For $\mathrm{LaI}_{3}, W$ reaches an approximate constant value at $1 \mathrm{keV}$, which is very similar to the value determined for CsI and $\mathrm{NaI}$ but is smaller than that obtained for $\mathrm{LaBr}_{3}$. The large discontinuities at $150 \mathrm{eV}$ and $700 \mathrm{eV}$ correspond to the photoelectric absorption of the $\mathrm{L}_{3}$ shell of $\mathrm{I}$ and $\mathrm{L}_{3}$ shell of $\mathrm{La}$.

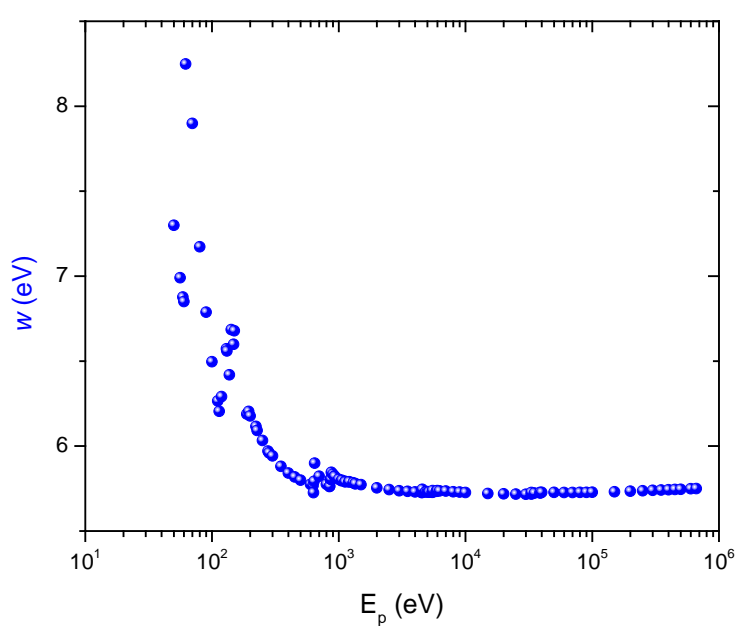

Fig. 8. Variation of the mean energy per electron-hole as a function of incident photon energy.

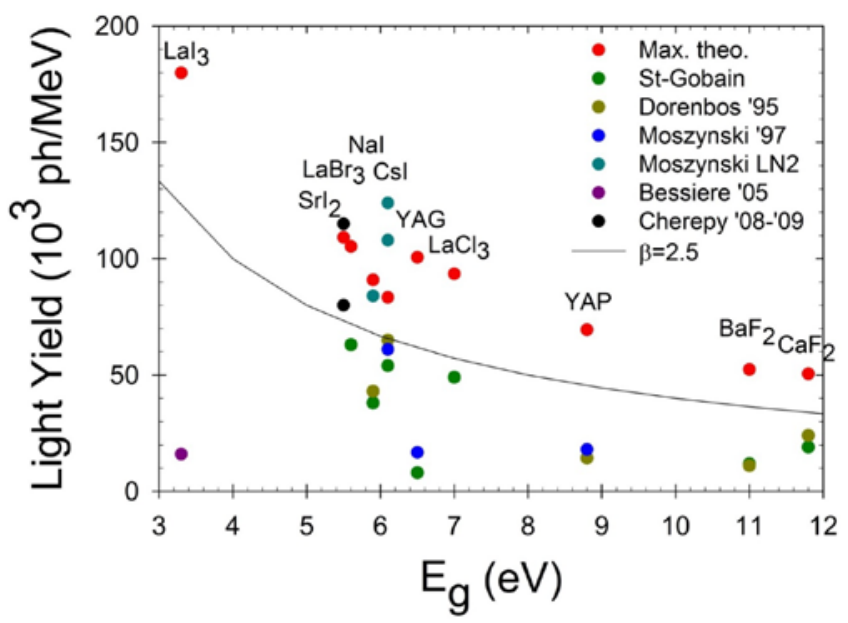

Fig. 9. Theoretical maximum light yields of a range of scintillators together with experimental values reported in the literature.

The calculated intrinsic properties of the lanthanum halide series are summarized in Table 2. $W$ is found to increase almost linearly with the band gap energy. $\mathrm{LaI}_{3}$ has the highest theoretical maximum light yield ( 174,000 ph/MeV), significantly higher than that of the widely-studied $\mathrm{LaBr}_{3}(\sim 105,000$ $\mathrm{ph} / \mathrm{MeV}$ ). To the best of our knowledge, the only measured light yield reported in the literature for (Cedoped) $\mathrm{LaI}_{3}$ is $\sim 16,000 \mathrm{ph} / \mathrm{MeV}$ at $100 \mathrm{~K}$ [Bessiere et al. Nucl. Instr. Meth. A 537 (2005) 22], i.e. only one tenth of the predicted theoretical maximum. The drastic quenching of the scintillation light in Cedoped $\mathrm{LaI}_{3}$ is thought to be due to the proximity of the $\mathrm{Ce}^{3+} 4 f^{0} 5 d^{1}$ excited state to the host CBM. In other words, autoionization of $\mathrm{Ce}^{3+}$ prevents any significant scintillation light from being emitted at room temperature. This finding led us to initiate a search for suitable dopants for $\mathrm{LaI}_{3}$, as presented in Task 1 . Fig. 8 compares all theoretical maximum light yields calculated to date to published experimental values. This plot shows that, in addition to $\mathrm{LaI}_{3}$, oxide scintillators such as YAG and YAP also have large potentials for improvement. Absolute light yields obtained for CsI at liquid nitrogen temperature appear to be overestimated with respect to our calculations. Absolute light yields are notoriously difficult to derive from experimental measurements. Therefore, our theoretical maximum light yields can be used as a guide for deriving experimental absolute light yields. 
Table 2. Intrinsic properties of scintillators in the lanthanum halide series, as calculated by NWEGRIM.

\begin{tabular}{cccccc}
\hline Material & $\begin{array}{c}\text { Band gap } \\
\text { energy (eV) }\end{array}$ & W (eV) & Fano factor & $\begin{array}{c}\text { Theo. max. } \\
\text { LY } \\
(\mathrm{ph} / \mathrm{MeV})\end{array}$ & $\begin{array}{c}\text { Main e-h creation } \\
\text { mechanisms (\%) }\end{array}$ \\
\hline $\mathrm{LaF}_{3}$ & 6.6 & 10.3 & 0.37 & 96,899 & Interband:75.1, plasmon:20.3 \\
$\mathrm{LaCl}_{3}$ & 7.0 & 10.7 & 0.25 & 93,458 & Interband:71.1, plasmon:24.0 \\
$\mathrm{LaBr}_{3}$ & 5.6 & 9.5 & 0.22 & 104,932 & Interband:72.1, plasmon:21.9 \\
$\mathrm{LaI}_{3}$ & 3.3 & 5.8 & 0.52 & 173,913 & Interband:78.8, plasmon:15.2 \\
\hline
\end{tabular}

\subsubsection{Elpasolite series}

Elpasolites are promising dual gamma and neutron detectors due to the halide elements and the light Li element they contain. The calculated intrinsic properties of the elpasolite series are shown in Table 3. The simulations show that elpasolite scintillators that contain $\mathrm{Br}$ (CLLB and CLYB) have higher theoretical light yields and larger Fano factors than those containing Cl (CLLC and CLYC).

Table 3. Intrinsic properties of scintillators in the elpasolite series, as calculated by NWEGRIM.

\begin{tabular}{cccccc}
\hline Material & $\begin{array}{c}\text { Band gap } \\
\text { energy }(\mathrm{eV})\end{array}$ & $\mathrm{W}(\mathrm{eV})$ & Fano factor & $\begin{array}{c}\text { Theo. max. } \\
\text { LY } \\
(\mathrm{ph} / \mathrm{MeV})\end{array}$ & $\begin{array}{c}\text { Main e-h creation } \\
\text { mechanisms (\%) }\end{array}$ \\
\hline CLLB & 6.0 & 9.7 & 0.35 & 103,093 & Interband:71.7, plasmon:19.8 \\
CLLC & 8.0 & 14.8 & 0.24 & 67,431 & Interband:65.6, plasmon:23.7 \\
CLYB & 5.7 & 9.5 & 0.32 & 105,374 & Interband:71.3, plasmon:20.1 \\
CLYC & 7.5 & 14.3 & 0.27 & 69,686 & Interband:71.6, plasmon:19.5 \\
\hline
\end{tabular}
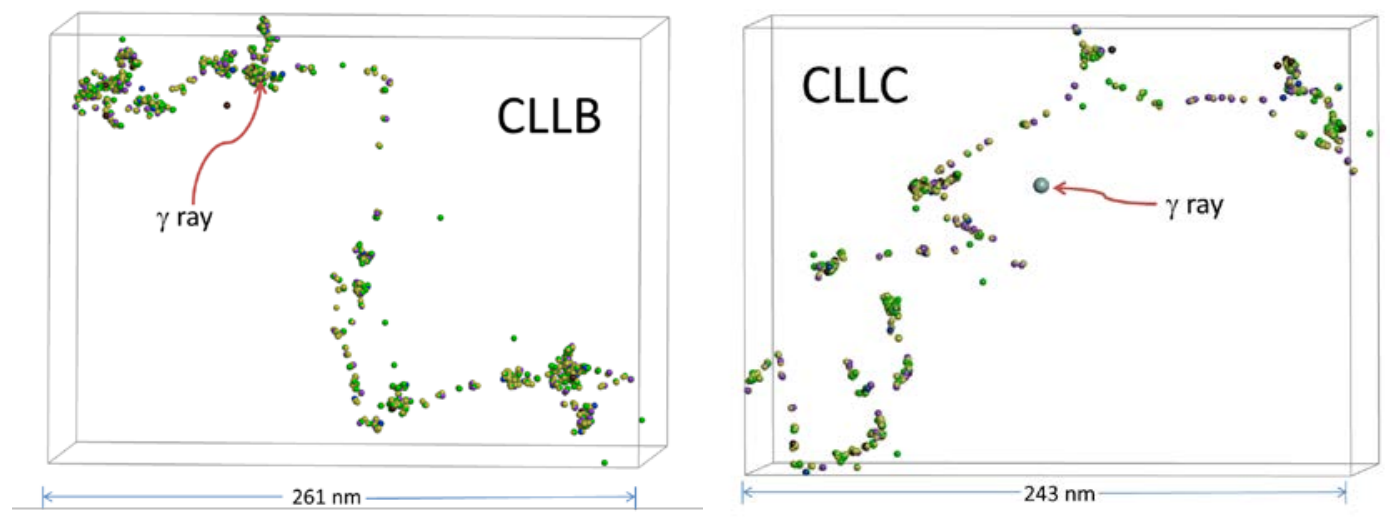

Fig. 10. Calculated spatial distributions of electron-hole pairs for a $10-k e V$ photon event in CLLB and CLLC. Holes are shown in yellow and electrons generated via interband transition, plasmon, ionization event, and relaxation are shown in green, red, blue, and purple, respectively.

Scintillation yield and energy resolution both strongly depend on the density of electronic excitation initially created in the track region. Fig. 9 shows typical distributions of electron-hole pairs created by a 10-keV incident photon in CLLB and CLLC. The electron-hole tracks show linear or clustered track sections, which could explain the differences among the Fano factors values of these materials. 


\subsubsection{Local principal curve analysis of ionization tracks}

A detailed understanding of ionization tracks is expected to enable quantitative predictions of the extent of nonproportionality in a range of scintillator materials. To date, a clear correlation between spatial structures of tracks and the intrinsic scintillation properties of these materials is still elusive. Therefore, a new activity initiated this FY consisted in using the Local Principal Curve Method to analyze the spatial structure of the electron-hole pair tracks produced in the energy cascade simulations. LPCM is an extension of the Principal Component Analysis approach, a well-known multivariate statistical technique in dimension reduction. LCPM generates smooth curves that pass through the centers (local means) of multidimensional data cloud. If the data clouds of the electron-hole pairs generated follow linear tracks and the tracks are captured by the LPCM algorithm, the distance of the data to the fitted curves will be small and the goodness-of-fit criterion $\left(R_{c}\right.$, in the range between 0 and 1$)$ based on such distance will be closer to 1 . On the other hand, if the distribution of electron-hole pairs form clusters without apparent track features, the fitted curves will not be a good representation of the data cloud and the large distances between the data and the fitted curve will lead to a goodness-of-fit value closer to 0 .

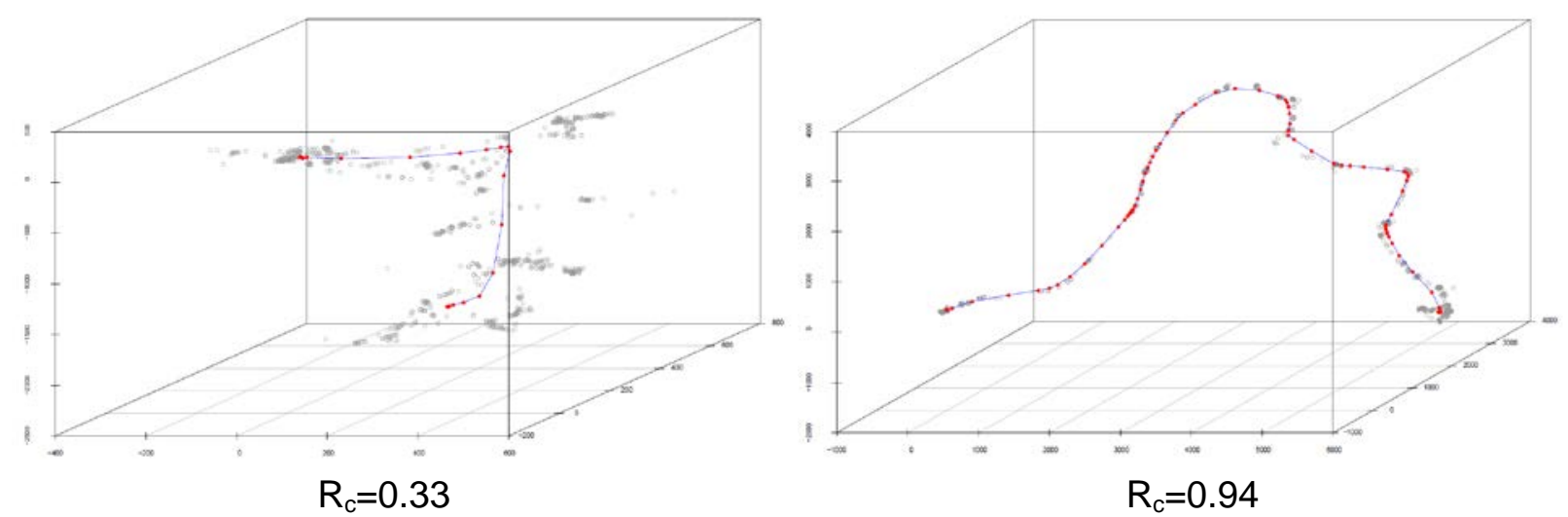

Fig. 11. Simulated spatial distributions of electron-hole pairs with local principal curves for two ionization tracks produced by $10-\mathrm{keV}$ photon events in CsI.

Fig. 10 shows an example of this analysis. The local principal curves (red dots linked with blue line) are superimposed onto the positions of electron-hole pairs (gray circles) produced by a 10-keV incident photon in two typical ionization tracks in CsI calculated by NWEGRIM. These plots show that LPCM traces the positions of electron-hole pairs very well $\left(\mathrm{R}_{\mathrm{c}}=0.94\right)$ in the case of the linear track while it does not capture the clustered nature of the other track $\left(\mathrm{R}_{\mathrm{c}}=0.33\right)$. Therefore, LCPM is a promising analysis approach for evaluating the spatial distribution of electron-hole pairs generated during the energy cascade. Further work to improve the reliability and efficiency of this analysis is on-going. 


\subsection{Task 3: Kinetics and efficiency of scintillation: nonproportionality, intrinsic energy resolution, and pulse shape discrimination}

\subsection{Summary of progress}

A paper describing our KMC model of scintillation in Tl-doped NaI was published in IEEE Transactions on Nuclear Science. A scintillation model of $\mathrm{SrI}_{2}$ that reproduces experimental data on the kinetics and efficiency of scintillation as a function of temperature, Eu concentration, and sample thickness was developed. The spatial and temporal scales of hot electron and hole thermalization in CsI and NaI were calculated based on group velocities obtained from band structure calculations carried out in Task 1.

\subsection{Publications/Presentations}

S. Kerisit, Z. Wang, R.T. Williams, J.Q. Grim, and F. Gao IEEE Transactions on Nuclear Science 61 (2014) 860

M. Prange, D. Wu, Y. Xie, L.W. Campbell, F. Gao, and S. Kerisit Proceedings of SPIE 9213 (2014) 92130L

(Invited) S. Kerisit Scintillation Mechanisms in Gamma Detectors, April 17 2014, Washington, DC

(Invited) S. Kerisit 2014 SPIE Meeting, August 19 2014, San Diego, CA

\subsection{Progress during FY14}

This FY, the work on scintillation mechanisms in Tl-doped NaI presented in the FY 2013 Annual Report was completed and summarized in a paper published in IEEE Transactions on Nuclear Science. Two additional activities were pursued under this task in FY14, namely the development of a KMC model of scintillation of $\mathrm{SrI}_{2}$ and the calculations of electron and hole thermalization in CsI and NaI.

\subsubsection{Scintillation model of $\mathrm{Srl}_{2}$}

Derivation of model parameters. Ab initio calculations carried out by our collaborators at LLNL suggest that STHs in $\mathrm{SrI}_{2}$ adopt a $\mathrm{V}_{\mathrm{k}}$-center structure through iodine dimerization, as is observed in alkali halides. Unlike alkali halides however, the same $a b$ initio calculations also suggest that electrons can selftrap through dimerization of two Sr atoms. In addition, STEs consist of neighboring STHs and STELs rather than an electron bound at a $\mathrm{V}_{\mathrm{k}}$-center as in alkali halides. The $a b$ initio calculations determined the delocalization energy for both STHs and STELs to be $0.1 \mathrm{eV}$, indicating that this energy constitutes the upper bound for the activation energy of STE hopping whereby the STE diffusion mechanism involves STH (STEL) delocalization and re-localization at a neighboring site followed by STEL (STH) delocalization and re-localization at a neighboring site of the STH (STEL). To the best of our knowledge, this value constitutes the sole estimation of the activation energy of STE hopping and is therefore used in our KMC model. 
Emission spectra of undoped $\mathrm{SrI}_{2}$ show a broad peak centered at approximately 560-570 nm [Alekhin et al. IEEE Trans. Nucl. Sci. 58 (2011) 2519; Sturm et al. IEEE Nucl. Sci. Symp. Conf. Rec. (2011) 7]. This peak is still visible at low levels of Eu doping but disappears as the $\mathrm{Eu}^{2+}$ concentration is raised [Alekhin et al. IEEE Trans. Nucl. Sci. 58 (2011) 2519]. Early interpretations of this peak assigned it to STE luminescence [Cherepy et al. IEEE Trans. Nucl. Sci. 56 (2009) 873]. However, Pustovarov et al. [Opt. Mater. 34 (2012) 926] showed that the intensity of this broad emission increased with time when $\mathrm{SrI}_{2}$ sample crystals were left under low vacuum at both 90 and $290 \mathrm{~K}$. As $\mathrm{SrI}_{2}$ is known to be highly hygroscopic and to easily form hydrates, Pustovarov et al. concluded that the broad emission peak was due to crystal hydrates that can form by reaction with residual water vapor. Therefore, we assign the broad peak to emission associated with oxygen impurities that result from the highly hygroscopic nature of $\mathrm{SrI}_{2}$ crystals. The X-ray excited emission spectra and decay times of pure $\mathrm{SrI}_{2}$ as function of temperature published by Alekhin et al. [Alekhin et al. IEEE Trans. Nucl. Sci. 58 (2011) 2519] were used to derive the rate parameters for radiative and non-radiative decay of the oxygen impurities, following the approach used previously for CsI [Kerisit et al. IEEE Trans. Nucl. Sci. 55 (2008) 1251].

In emission spectra of Eu-doped $\mathrm{SrI}_{2}$, Eu emission is typically observed as a single peak positioned at approximately $430 \mathrm{~nm}$ [Glodo et al. IEEE Trans. Nucl. Sci. 57 (2010) 1228]. Self-absorption of the Eu emission is a known characteristic of Eu-doped $\mathrm{SrI}_{2}$ and occurs because of the overlap between its excitation and emission spectra. The probability for self-absorption increases with increasing temperature, Eu concentration, and sample size, which makes the determination of the intrinsic radiative decay rate of Eu difficult. Lam et al. [IEEE Trans. Nucl. Sci. 59 (2012) 2052] measured the scintillation decay time of a $\mathrm{SrI}_{2}$ sample doped with $5.3 \pm 0.3 \% \mathrm{Eu}^{2+}$ and excited by $662 \mathrm{keV} \gamma$-rays from $295 \mathrm{~K}$ down to $5 \mathrm{~K}$ and found it to converge to $370 \pm 70 \mathrm{~ns}$ below approximately $50 \mathrm{~K}$. This result is consistent with the extrapolation of Alekhin et al. from a small $\mathrm{SrI}_{2}$ sampled doped with $0.5 \%$ Eu and excited with 370 -nm photons from 600 $\mathrm{K}$ to $80 \mathrm{~K}$, which yielded a decay time of $400 \mathrm{~ns}$. Therefore, the $\left(\mathrm{Eu}^{2+}\right)^{*}$ radiative decay rate was taken to be $2.5 \times 10^{6} \mathrm{~s}^{-1}$ in the KMC model.

Alekhin et al. applied a self-absorption model to the temperature dependence of their measured photoelectron yields and decay time constants for several $\mathrm{SrI}_{2}$ samples doped of $5 \% \mathrm{Eu}^{2+}$ and of varying thicknesses. In this model, an excited Eu ion, $\left(\mathrm{Eu}^{2+}\right)^{*}$, has a probability $\eta$ to emit a photon (=quantum efficiency) and emitted photon has a probability $a$ to be re-absorbed by a $\mathrm{Eu}^{2+}$ ion. Alekhin et al. obtained optimum agreement with their experimental data with $\eta=0.94$ and, in a subsequent publication on selfabsorption of $\mathrm{SrI}_{2}$ samples doped with 2\% Eu ${ }^{2+}$ [Alekhin et al. Nucl. Instrum. Meth. A 714 (2013) 13], they found a similar quantum efficiency $(\eta=0.95 \pm 0.05)$ to be successful in fitting the experimental data. Therefore, the $\left(\mathrm{Eu}^{2+}\right)^{*}$ non-radiative decay rate was taken to be $1.5 \times 10^{5} \mathrm{~s}^{-1}$ in the KMC model to reproduce the quantum efficiency derived by Alekhin et al.

Impurity concentration in Eu-doped $\mathbf{S r I}_{2}$. We first us the KMC model to investigate the relative intensity of the impurity and Eu emissions derived experimentally by Alehkin et al. for $\mathrm{SrI}_{2}$ samples doped with $\mathrm{Eu}^{2+}$ concentrations varying from 0.5 to $5 \%$ under X-ray excitation at $80 \mathrm{~K}$ and $300 \mathrm{~K}$. Because the impurity concentration in the samples is unknown, we used it as a variable to optimize the agreement between the experimental and calculated relative contributions of the impurity emission as a function of temperature and Eu concentration, while keeping all other KMC parameters fixed. Fig. 11 compares the results of the KMC simulations with experimental data. With increasing Eu concentration, the contribution of impurities to the light output decreases as the probability for STEs to trap at impurities decreases. Increasing the temperature also decreases the impurity contribution as it quenches more strongly than the Eu emission. There are two main sources of uncertainty in the experimental data: firstly, 
the Eu concentration was not directly measured but instead was assumed to be equal to the $\mathrm{EuI}_{2} / \mathrm{SrI}_{2}$ ratio in the starting material; and, secondly, the Eu and impurity contributions were obtained from fitting a Gaussian to each of the two peaks in the X-ray excited emission spectra but the goodness-of-fit of this fit was not reported.

Effect of temperature on light yield of $\mathrm{SrI}_{2}$ : Low $\mathrm{Eu}^{2+}$ concentration. To investigate the competition between Eu and impurity emissions, we simulate the temperature dependence of the light yield of $\mathrm{SrI}_{2}$ doped with a low Eu concentration. The KMC simulations were carried out at 80, 200, 300, 400, 500, and $600 \mathrm{~K}$, with a Eu concentration of $0.5 \%$, and with the impurity concentration determined in the previous section. Fig. 12 shows the total light yield, contribution from Eu emissions and contribution from impurity emission as a function of temperature. For comparison, the observations of Alekhin et al. on the temperature dependence of the integrated X-ray excited emission spectra of a $\mathrm{SrI}_{2}$ sample doped with $0.5 \% \mathrm{Eu}$ is also shown in Fig. 12. Good agreement between experiment and the simulations is obtained. The light yield from Eu emission does not show very strong temperature dependence in both simulations and experiments. As temperature increases, the difference between simulation and experimental results becomes more pronounced. The contribution from impurity emission, however, was quenched rapidly above $200 \mathrm{~K}$.

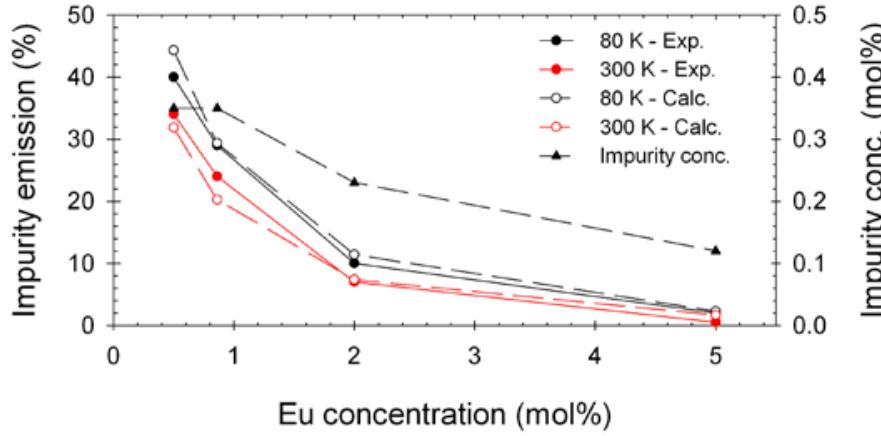

Fig. 12. Comparison of calculated and experimental percentages of the impurity emission in Eu-doped $\mathrm{SrI}_{2}$. Also shown is the impurity concentration required to optimize agreement with experimental data.

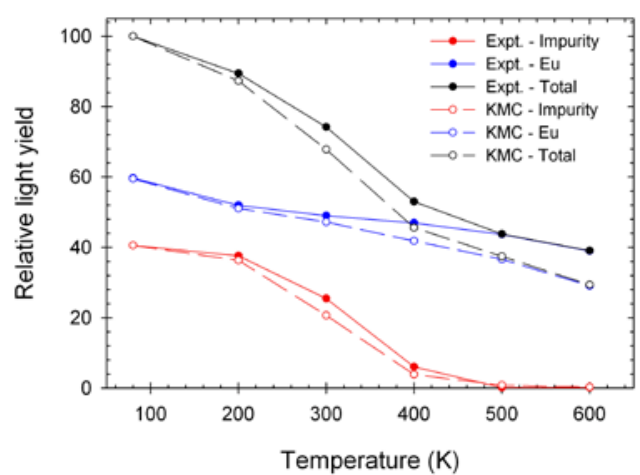

Fig. 13. Temperature dependence of the total light yield, light yield from $\left(\mathrm{Eu}^{2+}\right)^{*}$ emission, and light yield from defect emission, from experiment and KMC simulations.

\section{Effect of temperature and crystal thickness on kinetics of scintillation in $\mathrm{SrI}_{2}$ : $\mathrm{High} \mathrm{Eu}^{2+}$}

concentration. As the concentration of $\mathrm{Eu}^{2+}$ activators increases the contribution of impurities to the light output diminishes. We investigated the effect of temperature and crystal thickness on the kinetics of the light output of $\mathrm{SrI}_{2}$ at higher $\mathrm{Eu}^{2+}$ concentrations with the same set of parameters. Fig. 13 and Fig. 14 show the results obtained for $\mathrm{SrI}_{2}$ doped with $2 \%$ Eu. Overall, the simulated decay curves are in good agreement with experimental measurements. The decay time constant is lengthened with increasing temperature as well as sample thickness. The good agreement between the experimental data and our simulated results also indicates that our selected set of elementary processes and their corresponding parameters are adequate for modeling $\mathrm{SrI}_{2}$ scintillation for a broad range of conditions (i.e. Eu concentration, temperature, and sample thickness) 


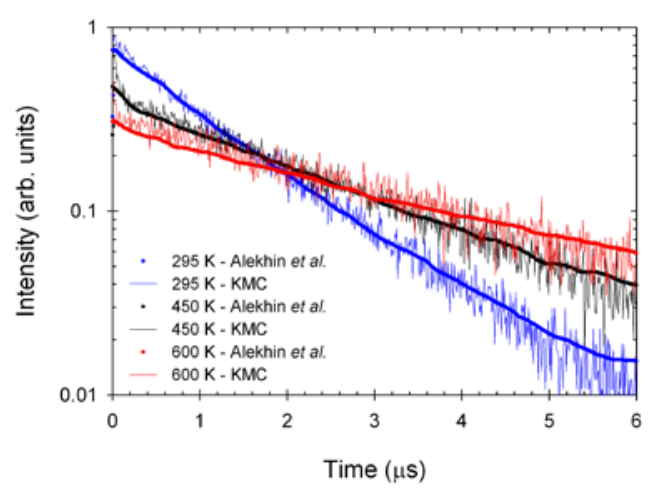

Fig. 14. Experimental and simulated scintillation decay curves of a $6-\mathrm{mm}$ thick $\mathrm{SrI}_{2}: 2 \%$ Eu crystals at 295,450 and $600 \mathrm{~K}$.

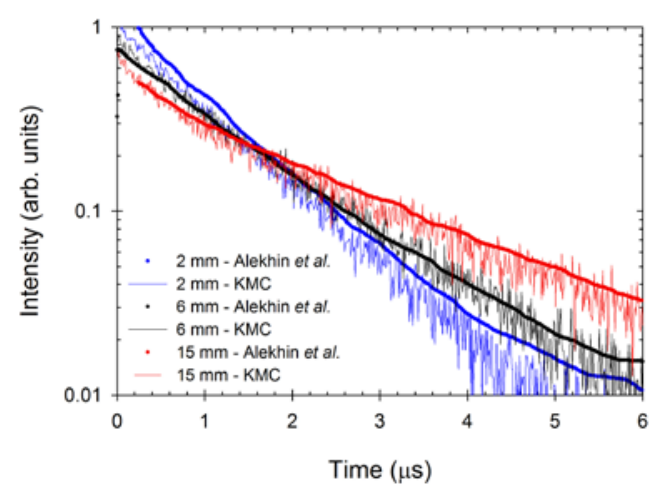

Fig. 15. Experimental and simulated decay curves of 2-mm, 6$\mathrm{mm}$, and $15-\mathrm{mm}$ thick $\mathrm{SrI}_{2}: 2 \% \mathrm{Eu}^{2+}$ crystals at $295 \mathrm{~K}$.

\subsubsection{Electron and hole thermalization in alkali halides}

In this section, we employed the group velocity model parameterized in Task 1 to calculate the spatial and temporal scales of electron and hole thermalization in CsI and NaI. For CsI, a comparison with the effective mass approximation and experimental data for the onset of STE formation is also presented. The calculations employed a semi-classical particle-phonon interaction model based on Fröhlich scattering for interaction with optical phonons and the formulation of Sparks et al. [Phys. Rev. B 24 (1981) 3519] for interactions with acoustic phonons.

Model validation. Experimental data on the length and time scales for electron thermalization in alkali halides are extremely rate. One recent study that offers a possible link between this work and experiment is the picosecond optical absorption study published by Williams and co-workers [Ucer et al. Phys. Rev. B 89 (2014) 165112]. In that work, a pump-probe technique was used whereby CsI was excited by two-photon absorption with excitation energies of 5.9 and $8.86 \mathrm{eV}$. Given a room-temperature band gap of CsI of approximately 5.8-5.9 eV, this results in the formation of free electrons with kinetic energies of approximately 0.1 and $3.0 \mathrm{eV}$ (assuming all the excess kinetic energy is assigned to the electrons). Time-resolved picosecond spectroscopy of the optical absorption induced by the two-photon absorption revealed a delay of $4 \mathrm{ps}$ in the onset of self-trapped exciton formation at $3.0 \mathrm{eV}$ relative to 0.1 $\mathrm{eV}$. Therefore, two simulations were performed for each model with electrons with initial kinetic energies of $0.1 \mathrm{eV}$ or $3.0 \mathrm{eV}$ and the delay in thermalization time between the two initial energies was compared with the value of 4 ps obtained experimentally. Because the experimental results are for ultraviolet excitation and initial random distribution of electron-hole pairs was used together with periodic boundaries conditions. A density of $10^{18}$ electron-hole pairs per $\mathrm{cm}^{3}$ was used as recommended in the work of Williams and co-workers. Ten simulations with 200 electron-hole pairs each were run in each case and electron-hole pair recombination was turned off to maximize statistics and concentrate on the intrinsic thermalization times. The thermalization time distributions thus obtained are shown in Fig. 15. At $0.1 \mathrm{eV}$, both models predict the large majorities of the electrons to reach thermal energy within $0.1 \mathrm{ps}$. At $3.0 \mathrm{eV}$, the time distribution peaks at approximately $3.4 \mathrm{ps}$ for the group velocity model whereas a broader distribution with a maximum at approximately 9 ps is obtained with the effective mass approximation model. These results indicate that the group velocity model yields good agreement with the time scale obtained experimentally whereas the effective mass approximation model overestimates the 
electron thermalization time. Therefore, the remainder of this report will focus on the group velocity model.

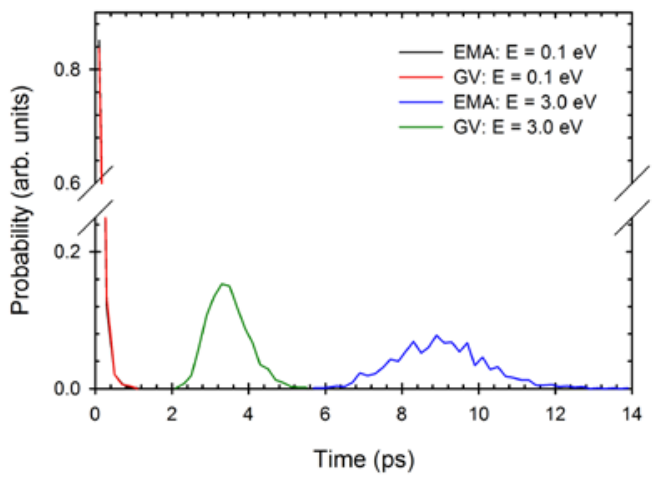

Fig. 16. Thermalization time distributions of electrons with kinetic energies of 0.1 or $3.0 \mathrm{~V}$ as obtained with the effective mass approximation (EMA) and group velocity (GV) models.

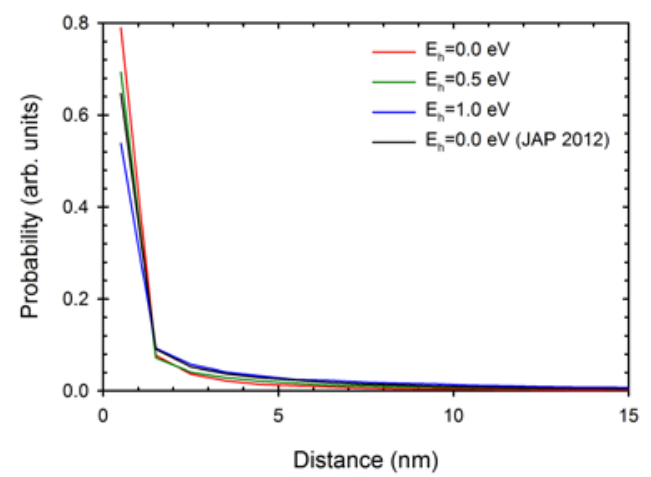

Fig. 17. Distance distributions of recombined electrons following irradiation of CsI with $2-\mathrm{keV} \gamma$-rays for three different initial kinetic energies of the holes $\left(E_{h}\right)$.

Electron and hole thermalization in CsI. A series of simulations was carried out using the electronhole pair energy and spatial distributions at the end of the energy cascade resulting from a 2-keV incident $\gamma$-ray in CsI, as calculated by NWEGRIM. These electron-hole pair distributions are the same as those used in a previous publication [Wang et al. J. Appl. Phys. 112 (2012) 014906]. 400 simulations with approximately 170 electron-hole pairs in each distribution were run, for a total of approximately 68000 electron-hole pairs. Because any excess energy is assigned to the electron following the creation of an electron-hole pair in the NWEGRIM calculations, the valence holes have no kinetic energy in the electron-hole pair distributions used as input for the thermalization calculations. However, as illustrated in Fig. 3, the valence band in CsI has a finite width of approximately $1.25 \mathrm{eV}$. Therefore, the initial kinetic energies of the holes in the thermalization calculations were varied from 0 to $1.25 \mathrm{eV}$. The kinetic energies of the electrons were scaled accordingly to conserve a constant overall initial kinetic energy.

In the simulations, particles with low initial kinetic energies or which are located in high-density regions of the tracks are able to recombine whereas those particles that do not recombine eventually reach thermal energy. The former are referred to as recombined particles and the latter as stopped particles. Recombination is very rapid and occurs within a time period not exceeding a few tenths of a picosecond. Distances traveled by electrons and holes before recombination are shown in Fig. 16 for three different initial kinetic energies of the holes $\left(\mathrm{E}_{\mathrm{h}}\right)$. The distributions are similar for electrons and holes and do not vary significantly with $E_{h}$. Fig. 16 shows that almost all the particles that recombine do so within approximately $5 \mathrm{~nm}$.

Thermalization distances of stopped electrons and holes are shown in Fig. 17. The thermalization distance is defined as the distance between the final (that is, at the point where the particle reaches thermal energy) and initial position of a given particle. As expected, electrons travel longer distances than holes because of their much lower effective mass. The distance distributions peak between 50 and $150 \mathrm{~nm}$ and extend to 400 to $500 \mathrm{~nm}$. In contrast, the holes thermalize over shorter distances with distance distributions that show a maximum at approximately $20 \mathrm{~nm}$. For both electrons and holes, the effect of $E_{h}$ on the distance distributions is small. 

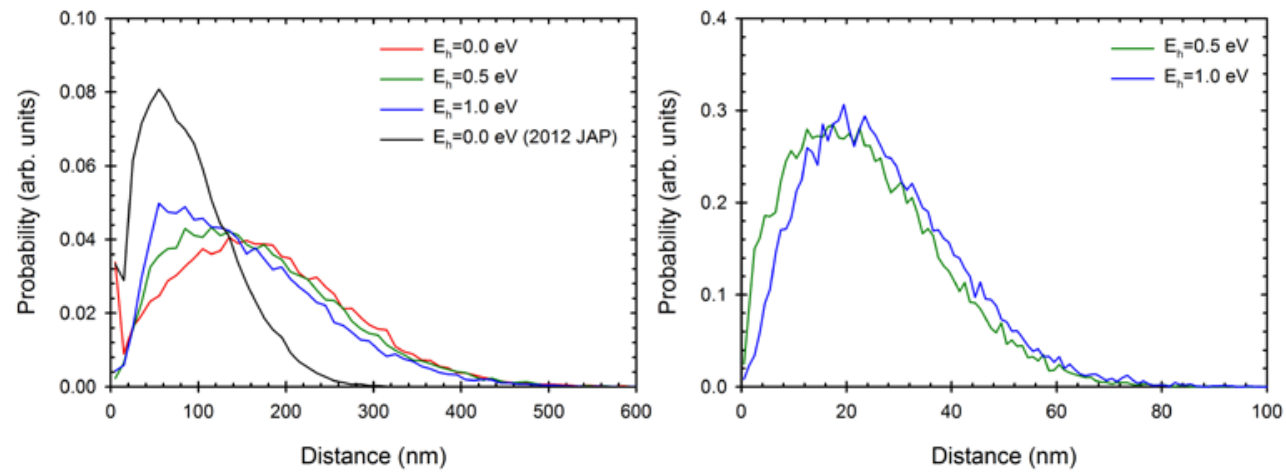

Fig. 18. Distance distributions of stopped electrons (left) and holes (right) following irradiation of CsI with 2-keV $\gamma$-rays for three different initial kinetic energies of the holes $\left(\mathrm{E}_{\mathrm{h}}\right)$. Also shown is the distance distribution obtained in previous work using an electron band mass of $1 \mathrm{~m}_{0}$ with polaron effects and $\mathrm{E}_{\mathrm{h}}=0 \mathrm{eV}$.

The thermalization time distributions of stopped electrons and holes are shown in Fig. 18. The thermalization time is defined as the time required for a given particle to reach thermal energy. As discussed above, the time during which electrons and holes persist as free particles directly affects the extent of third-order nonlinear quenching, which, in turn, plays a critical role in determining the magnitude of the deviation from proportional response of a given scintillator. This work indicates that hole thermalization is faster than electron thermalization, thus suggesting that the time scale for hole thermalization might constitute a controlling factor for the rate and magnitude of third-order nonlinear quenching.
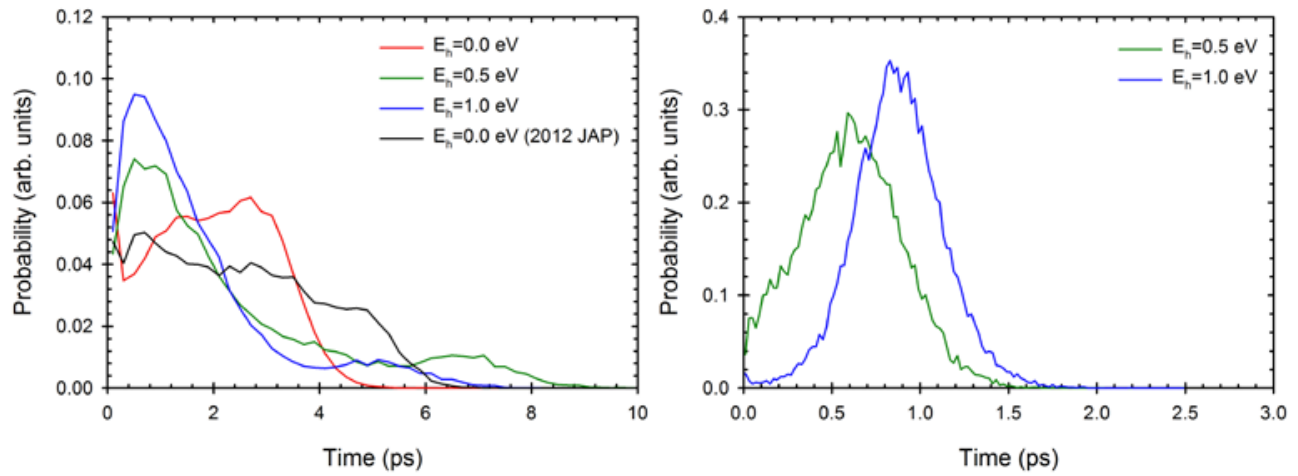

Fig. 19. Time distributions of stopped electrons (left) and holes (right) following irradiation of CsI with 2-keV $\gamma$-rays for three different initial kinetic energies of the holes $\left(E_{h}\right)$. Also shown is the distance distribution obtained in previous work using an electron band mass of $1 m_{0}$ with polaron effects and $E_{h}=0 \mathrm{eV}$.

Electron and hole thermalization in NaI. As was done for CsI, the band structure of NaI was calculated and used to derive the hole and electron group velocities and parameterize the KMC model. The same procedure as described above for CsI was employed to simulate electron and hole thermalization in NaI following irradiation with 2-keV $\gamma$-rays. The dependence of the thermalization distance and thermalization time of stopped electrons and holes on the kinetic energy of the holes is shown in Fig. 19 and Fig. 20. As for CsI, the thermalization times and distances of the electrons are greater than those of the holes. By and large, the thermalization times and distances in NaI are shorter than those calculated for CsI. This difference is due, for the most part, to the fact that the LO phonon 
energy is a key factor that affects the thermalization process and the fact that the LO phonon energy of $\mathrm{NaI}(0.023 \mathrm{eV})$ is greater than that of CsI $(0.01 \mathrm{eV})$.

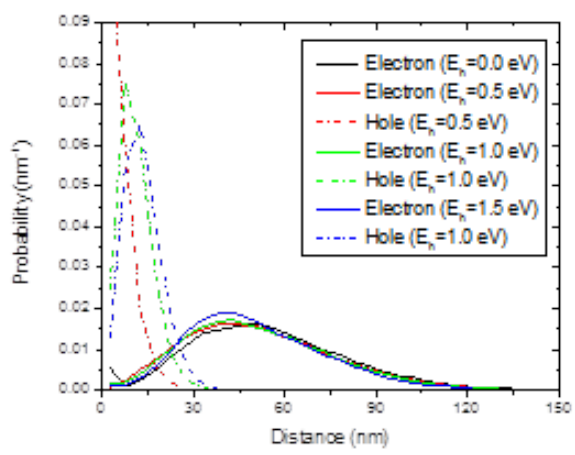

Fig. 20. Distance distributions of stopped electrons and holes following irradiation of $\mathrm{NaI}$ with $2-\mathrm{keV} \gamma$-rays for four different initial kinetic energies of the holes $\left(E_{h}\right)$.

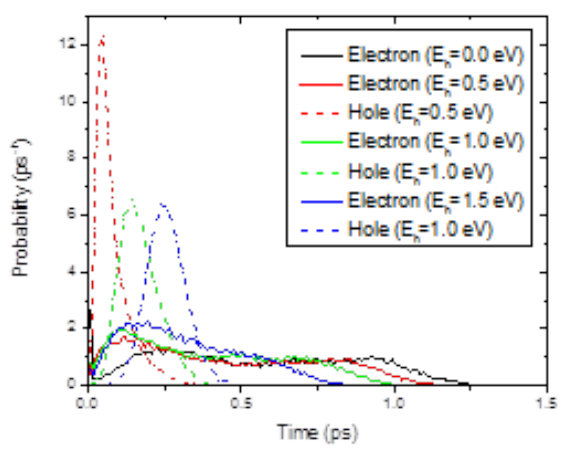

Fig. 21. Time distributions of stopped electrons and holes following irradiation of $\mathrm{NaI}$ with $2-\mathrm{keV} \gamma$-rays for four different initial kinetic energies of the holes $\left(E_{h}\right)$. 
Appendix A

\section{Publications and Invited Presentations}




\title{
Off-center Tl and Na dopant centers in CsI
}

\author{
R M Van Ginhoven ${ }^{1}$ and P A Schultz ${ }^{2}$ \\ ${ }^{1}$ Pacific Northwest National Laboratory, Richland, WA 99352, USA \\ ${ }^{2}$ Sandia National Laboratories, Albuquerque, NM, USA \\ E-mail: Renee.VanGinhoven@pnnl.gov and paschul@sandia.gov
}

Received 19 June 2013, in final form 28 August 2013

Published 6 November 2013

Online at stacks.iop.org/JPhysCM/25/495504

\begin{abstract}
We use density functional theory calculations to characterize the electronic and structural properties of the $\mathrm{Tl}$ and $\mathrm{Na}$ dopant centers in CsI. We find that the $\mathrm{Tl}$ and $\mathrm{Na}$ centers can accept one or two electrons and couple to long-range relaxations in the surrounding crystal lattice to distort strongly off-center to multiple distinct minima, even without a triplet excitation. The long-range distortions are a mechanism to couple to phonon modes in the crystal, and are expected to play an important role in the phonon-assisted transport of polarons in activated CsI and subsequent light emission in this scintillator.
\end{abstract}

(Some figures may appear in colour only in the online journal)

\section{Introduction}

There has been considerable interest in recent years in the development of new materials and optimization of current materials for use as scintillating radiation detectors. Signal production in scintillating detectors involves conversion of cascade energy into optical emission. This is a relatively slow process involving transport of carriers to luminescent centers, in competition with other processes that dissipate a portion of the initial energy. Prediction of the performance of new or improved materials requires an understanding of these transport processes. Cesium iodide doped with thallium or sodium (CsI:Tl, CsI:Na), is widely used as an effective scintillating material. Despite many careful studies over several decades [1], the mechanisms for energy transport and light emission, and the role of the dopant atoms in this material are not well understood. Previous cluster-based $a b$ initio calculations of $\mathrm{Tl}$ in $\mathrm{CsI}$ and $\mathrm{NaI}$ clusters have demonstrated the need for relatively large systems to adequately describe the surrounding lattice response, but only examined symmetric lattice distortions [2].

We use density functional theory (DFT) to investigate the electronic and structural properties of $\mathrm{Na}$ and $\mathrm{Tl}$ dopants in CsI, along with related intrinsic defects, to elucidate the behavior of these dopants. It is well established that standard DFT using semilocal exchange-correlation potentials does not correctly describe the expected energy carriers in the pure alkali halides, either self-trapped holes, also known as $\mathrm{V}_{k}$ centers, or self-trapped excitons (STE) [3, 4]. However, we are not attempting to model the STE or $\mathrm{V}_{k}$ center. For defects with well-localized electronic states, such as the vacancy and dopant impurity point defects we investigate here, we expect a semilocal description to provide a fully adequate and decisive description of the defect structure and behavior.

In addition to having a stable neutral state and a localized triplet excitation, a defect-trapped exciton (DTE), we find that each dopant center can accept one or two electrons. All of the charge states of substitutional $\mathrm{Tl}$ and $\mathrm{Na}$ distort off-center, and couple to surprisingly long-range distortions in the surrounding crystal lattice. Three symmetry-distinct distortions are found, into the 001-face, into the 110-edge, and along the 111-bond directions, and are stable even without having trapped a local triplet excitation (exciton). These distortions involve long-range lattice deformations that would naturally couple to phonon modes, and include local structural deformations that would facilitate capture and transport of excitons. This provides a novel mechanism to explain the emission behavior activated by the dopants.

\section{Computational methods}

The density functional supercell calculations for CsI:Tl were performed with the periodic pseudopotential SEQQUEST code [5]. The spin-polarized local-density approximation 
(LDA) calculations used the form parameterized by Perdew and Zunger [6] and the generalized gradient functional used was formulated by Perdew et al [7]. Carefully optimized [8] norm-conserving pseudopotentials [9] (PP) were used for all atoms: the cesium PP included its semicore $5 \mathrm{p}^{6}$ electrons among the valence electrons along with a non-linear core correction [10] $\left(R_{\mathrm{nlc}}=2.5 \mathrm{bohr}\right)$, and the [core] $5 \mathrm{p}^{6} 6 \mathrm{~s}^{0.1}$ atom used a hardened d-potential $\left(R_{l}=1.4\right)$ for its local potential; an otherwise standard $\mathrm{s}^{2} \mathrm{p}^{5}$ iodine PP used a hardened $l=3$ $\left(R_{l}=1.2\right)$ potential for the local potential as the optimal $l=2$ potential $\left(R_{l}=1.57\right)$ proved too soft for good transferability; and the $5 \mathrm{~d}^{10}$ shell of thallium was placed in the valence and used as the local potential, while its p-potential was tuned $\left(R_{l}=1.57\right)$ for better transferability. The double-zeta plus polarization basis sets were constructed of contracted Gaussian functions.

We used the local moment countercharge method to solve the Poisson equation for charged supercells [11] and extrapolated to bulk asymptotic limits using the finite defect supercell model [12]. Bulk screening energy outside the supercell [12] was evaluated using the experimental static dielectric constant [13], 5.65, and an internal screening depth of 1.8 bohr $(\sim 0.9 \AA)$. The numerical results are not highly sensitive to these values, and the convergence of results extrapolated from different sized supercells confirms the accuracy of this approach.

The defect calculations were performed with the supercell fixed at the theoretical equilibrium lattice parameter: $4.417 \AA$ for LDA and $4.680 \AA$ for PBE, the experimental lattice constant is $4.567 \AA$ [13]. The measured bulk modulus for B2 structure CsI is $12.6 \mathrm{GPa}$ [14]. Our computed LDA bulk modulus is $16.6 \mathrm{GPa}$, slightly stiffer than experiment, and the PBE value is $9.7 \mathrm{GPa}$, slightly softer than experiment, like the lattice constant, bracketing the experimental value as LDA and PBE usually do. Supercells ranged from $3 \times 3 \times 3$ (54-site) to $6 \times 6 \times 6(432$-site $)$ scaled versions of the primitive 2 -atom cell of the CsI B2 structure, and used $3^{3}$ grids for sampling the Brillouin zone in the 54-site cell and $2^{3} k$-grids for the larger cells. The atomic configurations were energy-relaxed to within $1 \mathrm{meV}$ of a local minimum, with forces on atoms

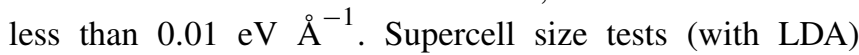
indicated that 54-site and 128-site failed to adequately contain the extensive strain fields around the defects, while 250-site results were well-converged compared to 432-site results. Results reported in this paper are extrapolated from 250-site supercell calculations. Formation energies are quoted in the Cs-rich limit, i.e., the bulk bcc Cs and CsI define the atomic reference chemical potentials. In this context, the computed formation energy of the neutral Cs vacancy is $3.74(4.22) \mathrm{eV}$ and of the I vacancy, 0.50 (0.54) eV using PBE (LDA). The heat of formation of B2 structure CsI, from bulk bcc Cs and the $\mathrm{I}_{2}$ molecule elemental references are computed to be $3.71 \mathrm{eV}$ in LDA and $3.42 \mathrm{eV}$ in PBE. These heats of formation, using the conventions outlined by Zhang and Northrup [15] to compute defect formation energies, can be used to convert the defect formation energies from one limit (Cs-rich) to another (I-rich).

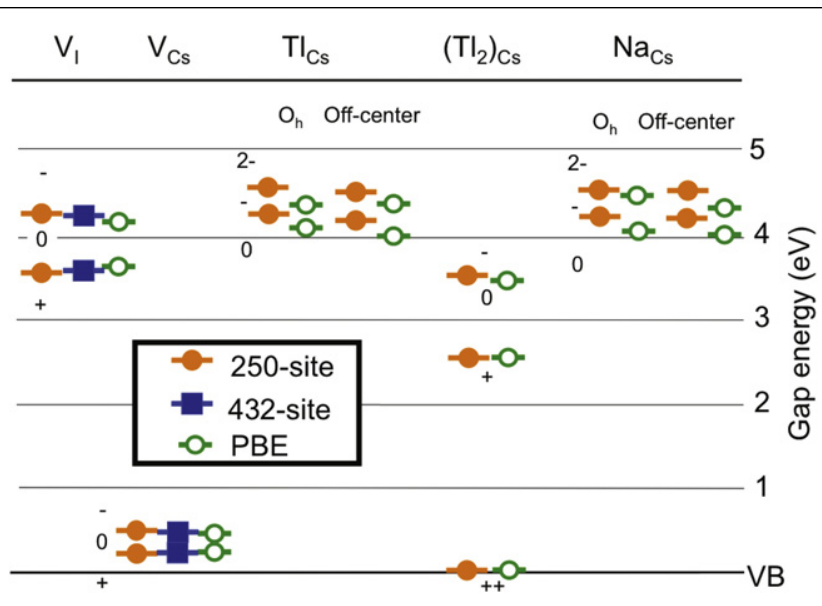

Figure 1. Defect levels in CsI. LDA and GGA (PBE) are both shown, results are the same within error of the techniques. Results are converged at 250 atoms.

\section{Results}

Calculations were performed for a purposefully selected set of defects, two intrinsic defects, the Cs vacancy $V_{C s}$ and the I vacancy $\mathrm{V}_{\mathrm{I}}$, and then for the dopant atoms $\mathrm{Tl}$ and $\mathrm{Na}$, both known to substitute on the $\mathrm{Cs}$ site: $\mathrm{Tl}_{\mathrm{Cs}}$ and $\mathrm{Na}_{\mathrm{Cs}}$. In Tl-doped CsI, the $\mathrm{Tl}$ dimer substitutional, $\left(\mathrm{Tl}_{2}\right)_{\mathrm{Cs}}$, is a common defect, and was also included in this series of calculations. Furthermore, this dimer proves especially important to set a useful limit on the position of the valence band edge. An extensive search was undertaken to find all the stable charge states and determine their optimum structure. The resulting defect level diagram, summarizing the positions of the ground state charge transitions within the CsI band gap, is presented in figure 1 .

The doubly ionized $\mathrm{Tl}$ dimer center state is only barely in the band gap in the DFT calculations, both LDA and PBE, and, in the absence of a more definitive marker to locate the band edge on this diagram, its $(+/++)$ transition is adopted as the valence band edge (VBE). The VBE cannot be directly computed in the same total energy calculations used to obtain the defect level energies, but we can use this defect, cleanly identified as a local defect state, and therefore in the band gap, as the upper bound of the position of the VBE. As we shall see later, this choice is likely overly conservative.

The $\mathrm{V}_{\mathrm{Cs}}$ has charge transitions only slightly above the $\left(\mathrm{Tl}_{2}\right)_{\mathrm{Cs}}(+/++)$ transition, near the VBE, and the $\mathrm{V}_{\mathrm{I}}$ has levels high in the gap. The near-perfect agreement between the levels extrapolated from the 250-site cells and from the 432-site cells, depicted in figure 1 for the vacancies, confirms the convergence of these defect level calculations to the infinitely dilute bulk limit. The LDA and PBE calculations agree closely with each other, finding the same charge states and similar ground state structures for all the defects. The computed levels for all defects are, reassuringly, not sensitive to the choice of density functional, lending greater confidence to the analysis of the results [8].

The measured band gap is reported to be $6.1-6.4 \mathrm{eV}$ [16-18]. Our computed defect levels span a range of almost 
$5 \mathrm{eV}$, exceeding the Kohn-Sham (eigenvalue) gap for CsI for LDA and PBE in these calculations, which is 3.80 and $3.58 \mathrm{eV}$, respectively.

The calculation of localized defect levels can be used to identify possible dopants, and defects that may compete with desired emitters for hole or electron trapping. For example, from figure 1 we can deduce that the iodine vacancy or the negatively charged $\left(\mathrm{Tl}_{2}\right)_{\mathrm{Cs}}$ may compete with the luminescent $\mathrm{Tl}$ or Na dopants, while the cesium vacancy certainly will not.

\subsection{Intrinsic defects}

The $\mathrm{V}_{\mathrm{I}}$ is a commonly occurring defect that plays an important role in energy losses [19], and interferes with light output. We find that this defect can trap either a hole or an electron, with 0,1 or 2 electrons in the vacancy for the $\mathrm{V}_{\mathrm{I}}(+), \mathrm{V}_{\mathrm{I}}(0)$ and $\mathrm{V}_{\mathrm{I}}(-)$ charge states of the defect (see figure 1). The neutral $\mathrm{V}_{\mathrm{I}}$, commonly known as the F-center, takes a symmetric $\mathrm{O}_{\mathrm{h}}$ structure in spin-polarized calculations, as do both the ionized $\mathrm{V}_{\mathrm{I}}(+)$ and the $\mathrm{V}_{\mathrm{I}}(-)$, the latter commonly known as the $\mathrm{F}^{\prime}$-center. Our calculations predict the ionization level for the F-center, the $(0 /+)$ transition in figure 1 , to be $3.5 \mathrm{eV}$ above the $\mathrm{Tl}$ dimer $(+/++)$ transition, our assumed VBE. The ionization level of the $\mathrm{F}^{\prime}$-center, the $(-/ 0)$ transition, is predicted to be $0.8 \mathrm{eV}$ above the F-center ionization, in excellent agreement with the experimental separation of $0.8 \mathrm{eV}$ [20]. This close agreement lends confidence to the calculations, but should be considered in light of large quoted uncertainties in the experimental analysis of $\sim 0.5 \mathrm{eV}$.

Locating the experimental levels relative to a band edge is less definitive, as this defect level position in the gap is not measured directly. The position of the F-center level is deduced through a combination of different experimental analyses, that starts with the photoexcitation of the F-center electron into vacuum, then derives the distance of the conduction band edge (CBE) from the vacuum via measurements of the electron affinity of bulk CsI, and then also includes the size of the band gap. The F-center electron and $\mathrm{F}^{\prime}$-center electron have vacuum ionization energies quoted as 2.2 and $1.4 \mathrm{eV}$, respectively, with large uncertainty in the latter [20]. The vacuum to CBE distance is quoted to be $0.3 \mathrm{eV}[20,18]$ to as large as $0.7 \mathrm{eV}$ [21]. The band gap also adds about $0.2 \mathrm{eV}$ uncertainty. The experimental analyses suggest an ionization level for the F-center of roughly or a little less than $4 \mathrm{eV}$ above the $\operatorname{VBE}[21,20,22]$. Our calculation predicts this level is $3.5 \mathrm{eV}$, or greater, above the VBE, in rather good agreement with this experimental analysis, considering the uncertainties in the experimental analysis. Given that the band gap is explicitly contained within the experimental assessment of this level, this implies that the effective band gap seen by the semilocal DFT calculations is within $\sim 0.5 \mathrm{eV}$ of experiment.

The low defect formation energy of the $\mathrm{V}_{\mathrm{I}}$ is consistent with the easy formation of F-centers [19, 23]. The formation energy of $\mathrm{V}_{\mathrm{I}}(+)$ is reduced linearly with the distance of the Fermi level below the F-center transition, where the $\mathrm{V}_{\mathrm{I}}(+)$ formation energy exactly matches the formation energy of $\mathrm{V}_{\mathrm{I}}(0)$. Taken together, the splitting of the $\mathrm{V}_{\mathrm{I}}$ donor states,

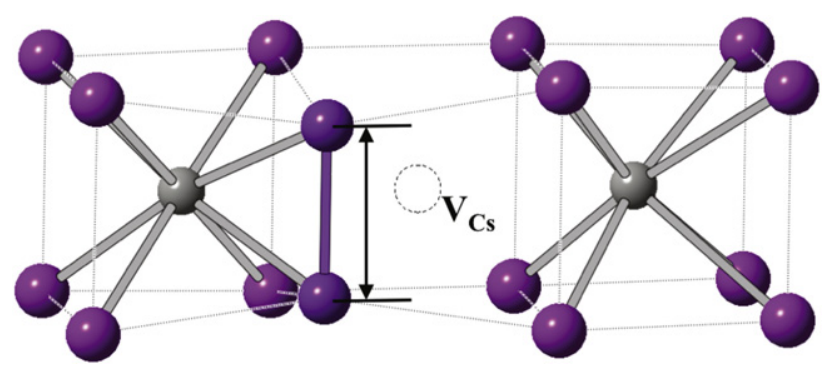

Figure 2. Local minimum with a $\mathrm{V}_{k}$-like distortion adjacent to a $\mathrm{Cs}$ vacancy $\left(\mathrm{V}_{\mathrm{Cs}}\right)$. Iodine is shown in dark gray (violet), Cs is light gray (silver). The iodine-iodine distance indicated by the arrow is decreased from the bulk crystal distance of 4.68-3.45 $\AA$.

the implicit agreement with experimental band gap, and the correct physical description of the formation of iodine vacancies, these results indicate that the semilocal LDA and PBE functionals are performing accurately for the ground state defects in CsI.

The $\mathrm{V}_{\mathrm{Cs}}$ may also trap either a hole or an electron. In LDA, the defect remains in a symmetric structure for each charge level. The relatively high formation energy, near $4 \mathrm{eV}$, is consistent with this not being a common defect in CsI. However, the $\mathrm{V}_{\mathrm{Cs}}$ exhibits another interesting feature in the calculations. Using spin-polarized PBE we found a second minimum for the neutral doublet state in which a pair of iodine atoms adjacent to the vacancy dimerize, resembling a $\mathrm{V}_{k}$ center geometry [3]. This minima is a shallow metastable state $0.18 \mathrm{eV}$ higher than the symmetric configuration. That a $\mathrm{V}_{k}$-like center is found at all without the use of hybrid functionals implies that this defect is a significant trap with the ability to strongly localize holes at sites in the iodine sublattice adjacent to the vacancy. This $\mathrm{V}_{k}+\mathrm{V}_{\mathrm{Cs}}$ configuration is shown in figure 2.

\subsection{Dopants}

Both the $\mathrm{Tl}$ and $\mathrm{Na}$ dopant centers, in addition to having a stable neutral state, can trap one or two electrons. In addition to these ground state structures, both the LDA and the PBE find a neutral triplet DTE to be stable for the $\mathrm{Tl}$ and $\mathrm{Na}$ dopants. These trap levels are high in the gap, roughly at or above the $V_{I}$ defect levels. While each charge state has a metastable symmetric on-site configuration, they all have lower-energy off-site distortions, with minima in three distinct directions: toward the 001-face, toward the 110-edge and a shallower minimum directly toward the nearest-neighbor iodine in the 111-direction. These distortions had been predicted for Tl-trapped excited states in CsCl:Tl, CsBr:Tl, and CsI:Tl [24-26]. Our calculations confirm the presence of these distortions in the triplet DTE, but we find that these distortions already exist in the ground state, including for the neutral defect.

The magnitudes of these displacements are summarized in table 1 . The distortion is small in the neutral defect (e.g. the $\mathrm{Tl}^{+}$), reminiscent of a soft-mode ferroelectric phase transition seen in dilated TII crystals [27]. This state has no localized 


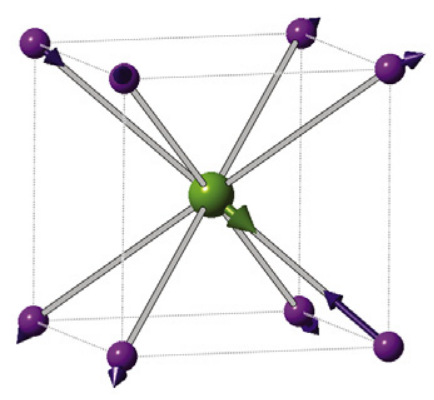

(a)

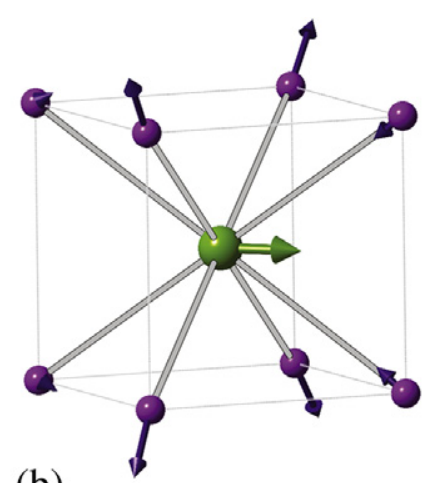

(b)

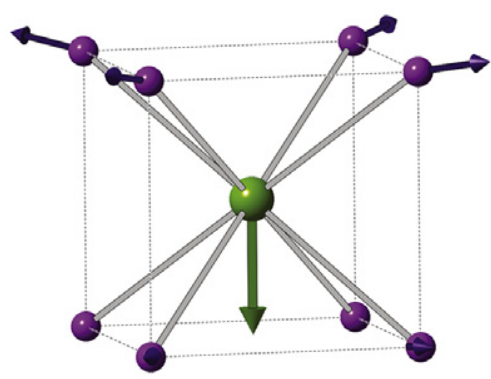

(c)

Figure 3. The three off-center structures for the $\mathrm{Tl}^{0}$ center and nearest-neighbor shell of iodine. $\mathrm{Tl}$ is shown as light gray (green), $\mathrm{I}$ is dark gray (violet). For visual clarity, the arrows are exaggerated by a factor of 2 for the Tl displacement, and by a factor of 10 for the I displacement. The $\mathrm{Tl}^{0}$ is displaced along (a) 100, (b) 110 or 111 directions. The displacements are with respect to the symmetric $\mathrm{Tl}^{0}$ structure.

Table 1. Displacement parameters in lattice units for $\mathrm{Tl}$ in $\mathrm{CsI}(\mathrm{Tl})$ for LDA and PBE in the neutral $\left(\mathrm{Tl}^{+}\right)$state, with $1\left(\mathrm{Tl}^{0}\right)$ and $2\left(\mathrm{Tl}^{-}\right)$ trapped electrons, and in the neutral triplet state.

\begin{tabular}{llllll}
\hline Direction & & $\mathrm{Tl}^{+}$ & $\mathrm{Tl}^{0}$ & $\mathrm{Tl}^{-}$ & Triplet \\
\hline 100 & LDA & 0.01 & 0.19 & 0.21 & 0.31 \\
& PBE & 0.11 & 0.23 & 0.23 & 0.36 \\
110 & LDA & 0.01 & 0.11 & 0.13 & 0.10 \\
& PBE & 0.10 & 0.15 & 0.14 & 0.16 \\
111 & LDA & 0.01 & 0.03 & 0.07 & 0.02 \\
& PBE & 0.09 & 0.08 & 0.07 & 0.08 \\
\hline
\end{tabular}

electrons in the gap, yet already exhibits a distinct distortion. The distortion is larger in PBE, perhaps because of larger lattice constant and softer bulk modulus in PBE, relative to the LDA, are more accommodating to an off-site distortion. The distortion becomes larger in magnitude as localized electrons are trapped at the center. The displacement for the $\mathrm{Tl}^{0}$ (single trapped electron) center is significant, about $1 \AA$, about halfway to the face of the cube formed by the nearest-neighbor iodine ions. The localization exhibited here demonstrates that the semilocal functionals, unlike for the STE and $V_{k}$ center, are not afflicted with a localization problem for these ground state defects.

The structures of the off-center dopants are only found if the surrounding lattice is allowed to relax; distorting the dopant off-site from a symmetric structure causes the dopant to return to the central site. The displacement is accompanied by significant long-range distortions in the lattice, involving the coordinated movement of over 100 atoms. The nature of the distortions for the dopant and nearby atoms is depicted in figure 3. The displacement field around the distorted dopant has pairing distortion reminiscent of incipient $\mathrm{V}_{k}$ centers. This dimerizing distortion is strongest and most apparent in the neighbors of the 110-distorted dopant center shown in figure $3(\mathrm{~b})$, but this tendency extends deep into the surrounding lattice.

In the neutral singlet ground state, the distorted configurations have energies within $0.02 \mathrm{eV}$ of each other. For dopants with trapped electrons or in the triplet state results for
Table 2. Calculated and measured triplet luminescence energy $\left(E_{\text {lum }}\right)$ for the $\mathrm{Tl}$ center in CsI, eV (nm).

\begin{tabular}{lllll}
\hline \multicolumn{2}{c}{ LDA (this work) } & & \multicolumn{2}{c}{ Experiment [30] } \\
\cline { 1 - 1 } \cline { 5 - 6 } Geometry & $E_{\text {lum }}$ & & Peak assignment & $E_{\text {lum }}$ \\
\hline 100 & $2.90(427)$ & & $A_{X}^{\prime}$ & $2.25(560)$ \\
110 & $3.38(367)$ & & $A_{T}^{\prime}$ & $2.55(490)$ \\
111 & $3.49(355)$ & & $A_{T}$ & $3.09(400)$ \\
$\mathrm{O}_{\mathrm{h}}$ & $3.55(349)$ & $A_{X}$ & $3.31(370)$ \\
\hline
\end{tabular}

LDA show that the off-center minima are $0.02-0.06 \mathrm{eV}$ lower than central position, all within $0.04 \mathrm{eV}$, in the order $111>$ $110>100$ (100 is lowest-energy position). For PBE, these energy differences are slightly enhanced. Despite requiring the concerted motion of over 100 atoms, barriers between these states are also low, $\sim 0.01 \mathrm{eV}$, close to the resolution of the current methods, for hops directly between off-center positions. The low barriers between the local minima are consistent with previous predictions from both theory end experimental work [24, 28] for energy transfer between the different excited state emissions assigned directly to the Tl. This result means that dopant atoms may vibrate nearly freely about the cage, except at very low temperatures, as was found for $\mathrm{Tl}$ in NaI:Tl [29]. It also implies that the position of the dopant is very strongly affected by local phonon modes or any local distortions.

3.2.1. Off-center $\mathrm{Tl}$ and calculated emission energies. The current results yield four possible Tl-related emission energies, based on the four possible positions of the $\mathrm{Tl}$ center in the triplet state. These energies, which we calculated as the difference between the triplet and singlet potential energy surfaces at the relaxed triplet (DTE) geometry, are listed in table 2, along with measured emission energies and suggested assignment based on polarization data [30]. The current results suggest emission from an interconnected potential energy surface of the several configurations of the $\mathrm{Tl}$ center. The calculated energies correspond roughly to the observed peaks, but this picture is not complete. 


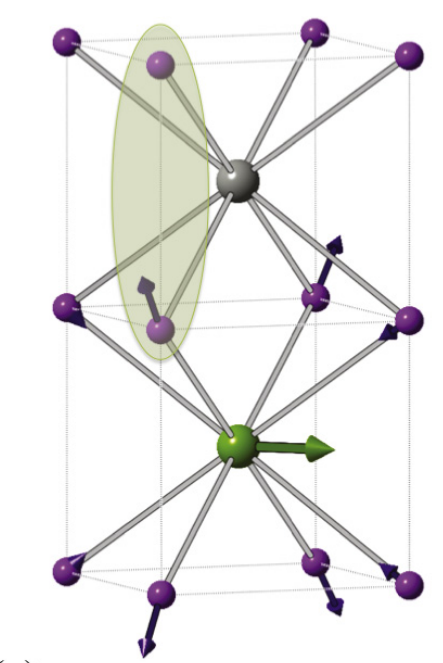

(a)

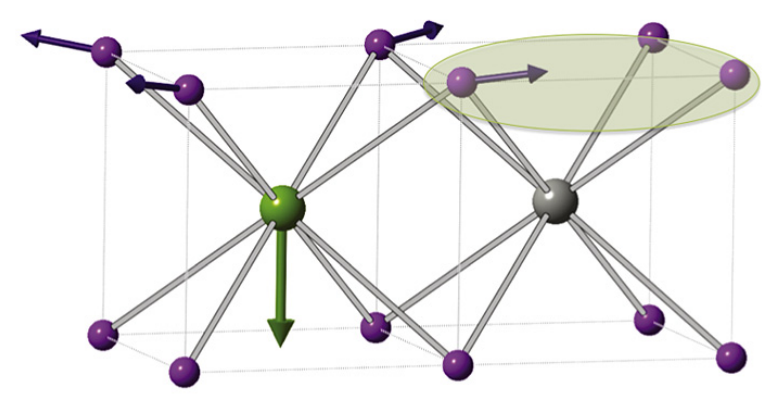

(b)

Figure 4. Possible $V_{k}$ center placements adjacent to 110 (a) and 100 (b) off-center Tl centers. Tl is shown as light gray (green), I is dark gray (violet), Cs is silver.

Interpretation of experimental measurements has led to the assignment of the UV peaks to relaxed excited states of the $\mathrm{Tl}$ center, and the visible emission peaks to tunneling recombination between the $\mathrm{Tl}$ atom and a nearby $V_{k}$ center [31, 32]. The two visible peaks, $\left(A_{X}^{\prime}\right.$ and $\left.A_{T}^{\prime}\right)$ are also postulated to be due to the interaction of a $\mathrm{Tl}$ and a perturbed STE either adjacent to the Tl, or offset by one unit cell [33].

Through examination of the distortions in the iodine sublattice in our results, we are able to identify several likely locations for a perturbed $V_{k}$ center. We note that especially for the 110 distorted $\mathrm{Tl}$, we see nearby distortions of the I lattice that resemble incipient $V_{k}$ centers even in the ground state. Proposed adjacent perturbed $\mathrm{V}_{k}$ centers for the 110 and $100 \mathrm{Tl}$ displacements are shown in figure 4 . There was no obvious candidate for the 111 distortion. Localization of an STE or $V_{k}$ on one of these iodine pairs should lower the energy of the 100 and 110 states, resulting in lower calculated emission energy, more in line with the experimental values. Quantitative investigation of the localized $\mathrm{V}_{k}$ center and STE, beyond the scope of this work, will require simulation methods with more explicit treatment of exchange, such as hybrid functionals $[29,3]$.

Finally, we note that the distortion field of the iodine sublattice resembles an array of incipient $\mathrm{V}_{k}$ centers, and therefore may be expected to interact with the transport of energy carriers near $\mathrm{Tl}$ or $\mathrm{Na}$ dopants. This indicates that transport and emission characteristics related to the off-center distortion of the dopant center will be strongly tied to phonon modes of the crystal.

\section{Acknowledgments}

This project is supported by Office of Nuclear Nonproliferation Research and Engineering (NA22), the US Department of Energy. The research was performed in part using EMSL, a national scientific user facility sponsored by the Department of Energy's Office of Biological and Environmental Research and located at Pacific Northwest National Laboratory. Sandia National Laboratories is a multi-program laboratory managed and operated by Sandia Corporation, a wholly owned subsidiary of Lockheed Martin Corporation, for the US Department of Energy's National Nuclear Security Administration under Contract DE-AC04-94AL85000.

\section{References}

[1] Nikl M 2000 Wide band gap scintillation materials: progress in the technology and material understanding Phys. Status Solidi 178595

[2] Aguado A, Ayuela A, Lopez J M and Alonso J A 1998 Lattice distortions around a $\mathrm{Tl}^{+}$impurity in $\mathrm{NaI}: \mathrm{Tl}^{+}$and CsI:Tl ${ }^{+}$scintillators: an $a b$ initio study involving large clusters Phys. Rev. B 5811964

[3] Van Ginhoven R M, Jaffe J E, Kerisit S and Rosso K M 2010 Trapping of polarons and excitons in scintillators: CsI, $\mathrm{LaCl}_{3}$, and $\mathrm{LaBr}_{3}$ IEEE Trans. Nucl. Sci. 57 2303-8

[4] Derenzo S E and Weber M J 1997 Ab initio cluster calculations of hole transport and activator excitation in CsI:Tl and CsI:Na IEEE CNF, Nuclear Science Symp. vol 1, p 164

[5] Schultz P A 1982 SeqQuest code unpublished (See http:/dft. sandia.gov/Quest)

[6] Perdew J P and Zunger A 1981 Self-interaction correction for density-functional approximations for many-electron systems Phys. Rev. B 235048

[7] Perdew J P, Burke K and Ernzerhof M 1996 Generalized gradient approximation made simple Phys. Rev. Lett. 773865

[8] Mattsson A E, Schultz P A, Mattsson T R, Leung K and Desjarlais M P 2005 Designing meaningful density functional theory calculations in material science-a primer Modelling Simul. Mater. Sci. Eng. 13 R1

[9] Hamann D R 1989 Generalized norm-conserving pseudopotentials Phys. Rev. B 402980

[10] Louie S G, Froyen S and Cohen M L 1982 Nonlinear ionic pseudopotentials in spin-density-functional calculations Phys. Rev. B 261738

[11] Schultz P A 2000 Charged local defects in extended systems Phys. Rev. Lett. 841942

[12] Schultz P A 2006 Theory of defect levels and the "band gap problem' in silicon Phys. Rev. Lett. 96246401 
[13] Bührer W and Hälg W 1971 Crystal dynamics of cesium iodide Phys. Status Solidi b 46679

[14] Chang Z P and Barsch G R 1967 Nonlinear pressure dependence of elastic constants and fourth-order elastic constants of cesium halides Phys. Rev. Lett. 191381

[15] Zhang S B and Northrup J E 1991 Chemical potential dependence of defect formation energies in GaAs: application to Ga self-diffusion Phys. Rev. Lett. 672339

[16] Eby J E, Teegarden K J and Dutton D B 1959 Ultraviolet absorption of alkali halides Phys. Rev. 116 1099-105

[17] Teegarden K and Baldini G 1967 Optical absorption spectra of the alkali halides at $10 \mathrm{~K}$ Phys. Rev. 155 896-907

[18] DiStephano T H and Spicer W E 1973 Photoemission from CsI: experiment Phys. Rev. B 71554

[19] Popov A I and Balanzat E $2000 \mathrm{~F}$ centre production in CsI and CsI-Tl crystals under $\mathrm{Kr}$ ion irradiation at $15 \mathrm{~K} \mathrm{Nucl}$. Instrum. Methods B 166/167 545-9

[20] Sarkas H W, Kidder L H and Bowen K H 1995 Photoelectron spectroscopy of color centers in negatively charged cesium iodide nanocrystals J. Chem. Phys. 102 57-66

[21] Chowdhury M A H, Watts S J, Imrie D C, McKemey A K and Holmes-Siedle A G 1999 Studies of radiation tolerance and optical absorption bands of $\mathrm{CsI}(\mathrm{Tl})$ crystals Nucl. Instrum. Methods A 432 147-56

[22] Galiy P V, Mel'nyk O Ya and Tsvetkova O V 2005 Excitonic ionizations of the electron centres in caesium iodide crystal and exoemission of electrons J. Lumin. 112 105-8

[23] Song K S and Williams R T 1996 Self-Trapped Excitons (Springer Series in Solid-State Sciences vol 105) (New York: Springer)

[24] Pazzi G P, Nikl M, Bacci M, Mihokova E, Hlinka J, Fabeni P and Salvini L 1994 Influence of $\mathrm{Ti}^{+}$concentration on emission and decay kinetics of CsI: $\mathrm{Ti}^{+}$single crystals J. Lumin. 60/61 527-30
[25] Zazubovich S G 1970 Jahn-Teller effect and partially covalent bond as causes of low symmetry of $\mathrm{Ga}^{+}, \mathrm{In}^{+}$, and $\mathrm{Tl}^{+}$ centers in CsCl-type alkali halides Phys. Status Solidi B 38119

[26] Bacci M, Mihóková E and Polák K 1997 Coexistence of Jahn-Teller distortions in an $\mathrm{O}_{\mathrm{h}}$ symmetry: a general view including the spin-orbit interaction Phys. Rev. B 5514257

[27] Du M-H and Singh D J 2010 Enhanced Born charge and proximity to ferroelectricity in thallium halides Phys. Rev. B 81144114

[28] Nikl M, Hlinka J, Mihokova E, Polak K, Fabeni P and Pazzi G P 1993 Decay kinetics of CsI:Tl luminescence excited in the A absorption band Phil. Mag. B $67627-49$

[29] Prange M P, Van Ginhoven R M, Govind N and Gao F 2013 Formation, stability and mobility of self-trapped excitations in $\mathrm{NaI}$ and $\mathrm{NaI}(\mathrm{Tl})$ Phys. Rev. B 87115101

[30] Nagirnyi V, Stolovich A, Zazubovich S, Zepelin V, Mihokova E, Nikl M, Pazzi G P and Salvini L 1995 Peculiarities of the triplet relaxed excited-state structure and luminescence of a CsI:Tl crystal J. Phys.: Condens. Matter $73637-53$

[31] Fu C-R, Chen L-Fu and Song K S 1999 Self-trapped excitons in pure and Na- and Tl-doped caesium halides and the recombination luminescence J. Phys.: Condens. Matter $115517-32$

[32] Mihokova E, Nagirnyi V, Nikl M, Stolovich A, Pazzi G P, Zazubovich S and Zepelin V 1996 Relaxed excited state structure and luminescence of thallium-doped caesium chloride and bromide J. Phys.: Condens. Matter 8 4301-14

[33] Nagirnyi V, Zazubovich S, Zepelin V, Nikl M and Pazzi G P 1994 A new model for the visible emission of the CsI:Tl crystal Chem. Phys. Lett. 227 533-8 


\title{
Calculation of energy relaxation rates of fast particles by phonons in crystals
}

\author{
M. P. Prange, ${ }^{1, *}$ L. Campbell, ${ }^{2}$ and F. Gao ${ }^{1}$ \\ ${ }^{1}$ Fundamental and Computational Sciences Directorate, \\ Pacific Northwest National Lab, Richland, WA 99354 \\ ${ }^{2}$ National Security Directorate, Pacific Northwest National Lab, Richland, WA 99354
}

(Dated: September 17, 2014)

\begin{abstract}
We present $a b$ initio calculations of the temperature-dependent exchange of energy and momentum between a classical charged point-particle of arbitrarily large velocity and the phonons of a crystalline material. The phonons, which are computed using standard density functional perturbation theory (DFPT) methods, interact with the moving charge via the Coulomb interaction between the density induced in the material by phonon excitation and the charge of the classical particle. Energy and momentum relaxation rates are computed using standard time-dependent perturbation theory. The method, which is applicable wherever DFPT is, is illustrated with results for several simple materials. We elucidate the relationship of the current theory with existing approaches to the electron-phonon interaction.
\end{abstract}

PACS numbers:

\section{INTRODUCTION}

When a material undergoes high energy excitation (e.g. by irradiation by high energy photons or ions), the subsequent relaxation produces a large number of electrons and holes which propagate. If the material has a gap in the spectrum of electronic excitations, particles with kinetic energy smaller than the gap can not lose energy by electronic excitation. In this regime, the particles still exchange energy with the vibrational degrees of freedom. Most treatments of the electron-phonon interaction focus on low energy carriers confined to a single band in small regions of $\mathbf{k}$-space. The calculations presented here are complementary to these methods and focus instead on the cooling of high-energy electrons by the lattice. We employ a simple semi-classical theory in which the field of a charged particle (moving with a specified velocity) is treated as a perturbation to the vibrational modes of the crystal. We present numerical calculations of the power transferred between the lattice and perturbing charge that are based on density functional perturbation theory calculations of the density response to lattice distortions.

\section{SEMICLASSICAL TREATMENT OF THE ELECTRON-PHONON INTERACTION}

In this paper we analyze a model of the interaction of a crystal with an external point particle of charge $Z$ at a time-dependent position $\mathbf{r}(t)$. The fundamental quantities in this analysis are the positions $\left\{\mathbf{R}_{s, \kappa}(t)\right\}$ of the nuclei of the atoms that comprise the crystal. The subscript $s$ refers to the unit cell and $\kappa$ refers to a particular sublattice. The crystal has $N$ repeated unit cells which occupy a volume $V=N \Omega$. These nuclear positions (and their conjugate momenta) are quantum mechanical operators; the Hamiltonian of our theory is the kinetic energy of the nuclear motion plus the ground state potential energy surface which depends only on the nuclear positions.
As the perturbing particle moves through the lattice, it exerts forces on the crystal, which responds by deforming. We assume the interaction energy is given by

$$
H_{\text {int }}(t)=Z \int d^{3} r^{\prime} \frac{\delta n_{\text {tot }}\left(\mathbf{r}^{\prime}\right)}{\left|\mathbf{r}(t)-\mathbf{r}^{\prime}\right|},
$$

where

$$
\begin{aligned}
& \delta n_{\text {tot }}(\mathbf{r})=\delta n_{\text {nuc }}(\mathbf{r})+\delta n_{\text {elec }}(\mathbf{r}) \\
&=\sum_{s, \kappa} Z_{\kappa}\left[\delta^{3}\left(\mathbf{r}-\mathbf{R}_{s, \kappa}\right)-\delta^{3}\left(\mathbf{r}-\mathbf{R}_{s, \kappa}^{(0)}\right)\right] \\
&-\left[n\left(\mathbf{r} ;\left\{\mathbf{R}_{s, \kappa}\right\}\right)-n\left(\mathbf{r} ;\left\{\mathbf{R}_{s, \kappa}^{(0)}\right\}\right)\right]
\end{aligned}
$$

is the change in the ground state charge density of the crystal (electrons and nuclei) induced by displacing the nuclei from their equilibrium positions $\left\{\mathbf{R}_{s, \kappa}^{(0)}\right\}$ to positions $\left\{\mathbf{R}_{s, \kappa}\right\}$.

We write the Hamiltonian for our model in terms of annihilation and creation operators for phonon modes:

$$
\begin{aligned}
H & =H_{0}+H_{1} \\
& =\sum_{\lambda, \mathbf{q}} \omega_{\lambda, \mathbf{q}}\left(a_{\lambda, \mathbf{q}}^{\dagger} a_{\lambda, \mathbf{q}}+\frac{1}{2}\right)+\sum_{\lambda, \mathbf{q}} H_{\lambda, \mathbf{q}}(t)\left(a_{\lambda,-\mathbf{q}}^{\dagger}+a_{\lambda, \mathbf{q}}\right) .
\end{aligned}
$$

In the sums here, $\mathbf{q}$ runs over the Brillouin zone of the crystal and $\lambda$ over the phonon branches of which there are thee times the number of sublattices. The time dependent coupling

$$
H_{\lambda, \mathbf{q}}(t)=\sum_{\mathbf{G}} \frac{(4 \pi Z)}{|\mathbf{q}+\mathbf{G}|^{2}} n_{\lambda}(\mathbf{q}+\mathbf{G}) e^{i(\mathbf{q}+\mathbf{G}) \cdot \mathbf{r}(t)}
$$

is the Coulomb interaction between the charge density $n_{\lambda}(\mathbf{q}+\mathbf{G})$ induced at wavevector $\mathbf{q}+\mathbf{G}$ by excitation of the phonon mode $(\mathbf{q}, \lambda)$ (which has annihilation and 


\title{
Experimental and computational results on exciton/free-carrier ratio, hot/thermalized carrier diffusion, and linear/nonlinear rate constants affecting scintillator proportionality
}

\author{
R. T. Williams*a, Joel Q. Grimª, Qi Li ${ }^{\mathrm{a}}$, K. B. Ucer ${ }^{\mathrm{a}}$, G. A. Bizarri ${ }^{\mathrm{b}}$, S. Kerisit ${ }^{\mathrm{c}}$, Fei Gao ${ }^{\mathrm{c}}$, P. \\ Bhattacharya $^{\mathrm{d}}$, E. Tupitsyn ${ }^{\mathrm{d}}$, E. Rowe ${ }^{\mathrm{d}}$, V. M. Buliga ${ }^{\mathrm{d}}$, and A. Burger ${ }^{\mathrm{d}}$ \\ ${ }^{a}$ Department of Physics, Wake Forest University, Winston-Salem, NC 27109 \\ ${ }^{\mathrm{b}}$ Lawrence Berkeley National Laboratory, Berkeley, CA 94720 \\ ${ }^{c}$ Pacific Northwest National Laboratory, Richland WA 99352 \\ ${ }^{\mathrm{d}}$ Department of Physics, Fisk University, Nashville, TN 37208
}

\begin{abstract}
Models of nonproportional response in scintillators have highlighted the importance of parameters such as branching ratios, carrier thermalization times, diffusion, kinetic order of quenching, associated rate constants, and radius of the electron track. For example, the fraction $\eta_{e h}$ of excitations that are free carriers versus excitons was shown by Payne and coworkers to have strong correlation with the shape of electron energy response curves from Compton-coincidence studies. Rate constants for nonlinear quenching are implicit in almost all models of nonproportionality, and some assumption about track radius must invariably be made if one is to relate linear energy deposition $\mathrm{dE} / \mathrm{dx}$ to volume-based excitation density $n\left(\mathrm{eh} / \mathrm{cm}^{3}\right)$ in terms of which the rates are defined. Diffusion, affecting time-dependent track radius and thus density of excitations, has been implicated as an important factor in nonlinear light yield. Several groups have recently highlighted diffusion of hot electrons in addition to thermalized carriers and excitons in scintillators. However, experimental determination of many of these parameters in the insulating crystals used as scintillators has seemed difficult. Subpicosecond laser techniques including interband z scan light yield, fluence-dependent decay time, and transient optical absorption are now yielding experimental values for some of the missing rates and ratios needed for modeling scintillator response. First principles calculations and Monte Carlo simulations can fill in additional parameters still unavailable from experiment. As a result, quantitative modeling of scintillator electron energy response from independently determined material parameters is becoming possible on an increasingly firmer data base. This paper describes recent laser experiments, calculations, and numerical modeling of scintillator response.
\end{abstract}

Keywords scintillator, nonproportionality, light yield, nonlinear quenching, diffusion, hot electron

\section{INTRODUCTION}

The paired alternatives for the scintillator material characteristics listed in the title of this paper anticipate the Conclusion, in which predictive trends in scintillator nonproportionality and light yield follow a succession of branch points according to values of a few material parameters. Payne et al $[1,2]$ concluded from empirically modeled electron energy response data from Compton-coincidence light yield that the free-carrier fraction denoted $\eta_{e h}$ is an important parameter affecting proportionality. One could alternatively talk in terms of the exciton fraction $\left(1-\eta_{e h}\right)$. We shall see that $\eta_{e h}$ is a function of time and electron temperature, but a single-valued $\eta_{e h}$ parameter can be associated with the average value of the evolving $\eta_{e h}\left(T_{e}, t\right)$ during nonlinear quenching. Hence, the first of the branch points according to exciton/free-carrier ratio can be tested for its correlation with scintillator proportionality if we have some way of measuring $\eta_{e h}$ independent of fitting a proportionality curve. We will describe such an experiment on laser interband Photon Density Response (PDR) in this paper, alongside results from the literature on the more traditional Electron Energy Response (EER) data.

PDR is a measurement of light yield versus absorbed photon density, where each ultraviolet photon produces one interband excitation. Thus PDR is a measure of light yield (or conversely, nonlinear quenching) as a function of excitation density. EER, on the other hand, is a measurement of light yield versus initial electron energy of a Compton electron [1,2] or K-shell photoelectron [3]. In EER, lower initial electron energy implies higher average excitation density, and one sometimes interprets the EER curves as qualitatively indicating how light yield changes with changing

Hard X-Ray, Gamma-Ray, and Neutron Detector Physics XV, edited by Michael Fiederle, Arnold Burger, Larry Franks, Ralph B. James, Proc. of SPIE Vol. 8852, 88520J · (c) 2013 SPIE CCC code: $0277-786 X / 13 / \$ 18 \cdot$ doi: $10.1117 / 12.2027716$ 
excitation density. But of course the correspondence is complicated due to the fluctuating distribution of excitations created by slowing of the high energy electron, the fact that scintillation light yield is integrated over the entire trajectory of a slowing primary electron and its secondaries, and the strong radial concentration gradient of excitations (mainly charge carriers) produced in the track. Extracting parameter values such as free-carrier fraction, the nonlinear rate constants, and diffusion coefficients (cool and hot) from the complex track structures contributing to EER data is thus very model dependent. In contrast, the horizontal axis in PDR plots is simply excitation density occurring on-axis just inside the sample surface. Furthermore, the energy imparted to each electron-hole pair by absorption of an ultraviolet interband photon is the same and can be tuned. Finally, the gradients of excitation density produced by interband laser absorption are at least an order of magnitude smaller than the gradient of excitation density around an electron track. When carrier mobilities are low or modest, as in most scintillators, we can neglect diffusion effects in the laser PDR geometry in order to deduce dependence of light yield directly on excitation density. From this dependence, one can extract nonlinear quenching rate constants as well as free-carrier fraction $\eta_{e h}$ by fitting a rate equation to the PDR curve. With measured values of the free-carrier fraction and/or exciton fraction as well as the nonlinear quenching rate constants in hand from PDR experiments, one can then include carrier and/or exciton diffusion in the full rate equation needed to describe the electron track environment and predict electron energy response for comparison to EER measurements. Because nonlinear quenching goes on during the time of hot carrier thermalization in some scintillators, it has been found [4-8] that one needs to account for diffusion coefficients that are functions of electron temperature and therefore indirectly functions of time. This requires calculations and modeling to take into account.

\section{EXPERIMENT}

The experiment for measuring photon density response is represented schematically in Fig. 1. The experiment and analysis has been described in detail in Ref. [9], where it was called "interband z scan" because of similarity of the experimental setup to the well-known z-scan method of measuring optical nonlinearities of a sample through effects on the transmitted beam as a function of focus.[10] In the interband $\mathrm{z}$ scan experiment, the laser beam is totally absorbed within $\sim 100 \mathrm{~nm}$ of the sample face and luminescence is detected as indicated in Fig. 1.

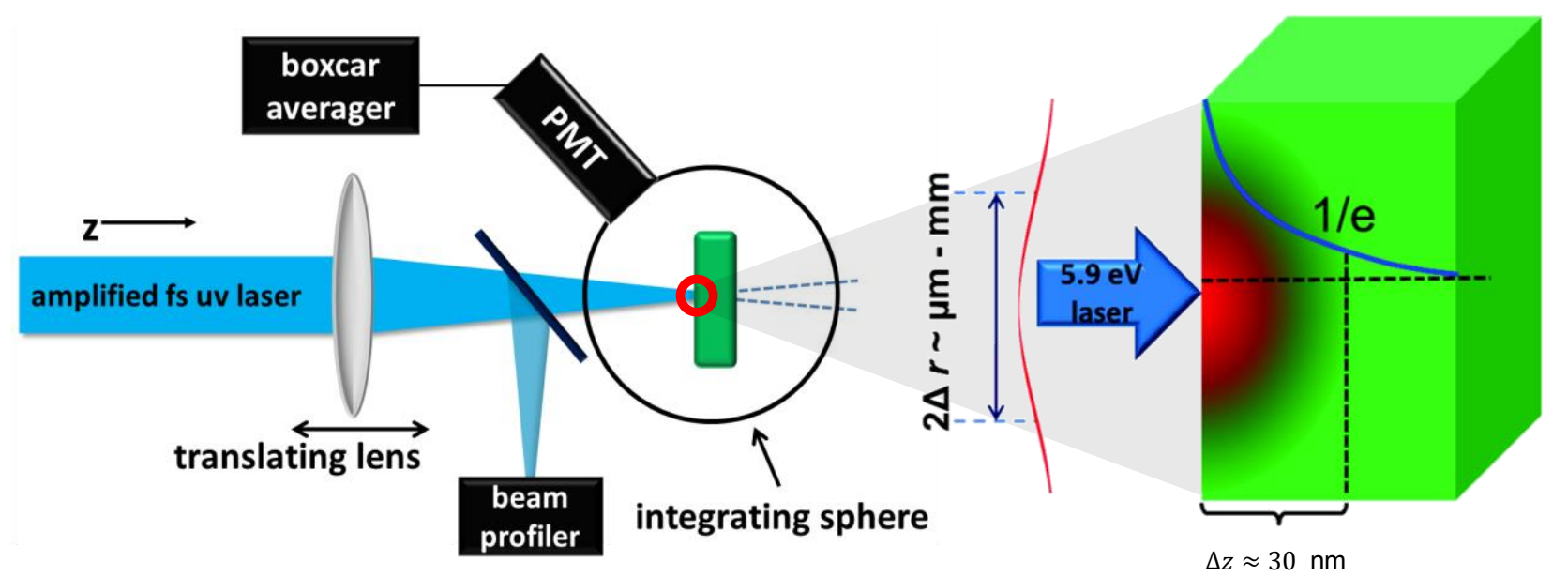

Figure 1. Experimental schematic for interband laser z scan or PDR experiment is shown in the left half. The right half is an enlargement of the sample showing the exponential attenuation profile in the longitudinal direction and the Gaussian beam profile in the transverse plane of the sample surface.

Having acknowledged the setup similarity to conventional z scan measurements, we will henceforth refer to the experiment as photon density response (PDR) since this places it more accurately with respect to other well-known scintillator measurements including electron energy response,[1,2] photon energy response,[11] and gamma energy response.[12][9] In PDR, light yield is measured as a function of excitation density under condition of constant excitation number. If there is no nonlinearity of light yield, the PDR curve should be flat. 

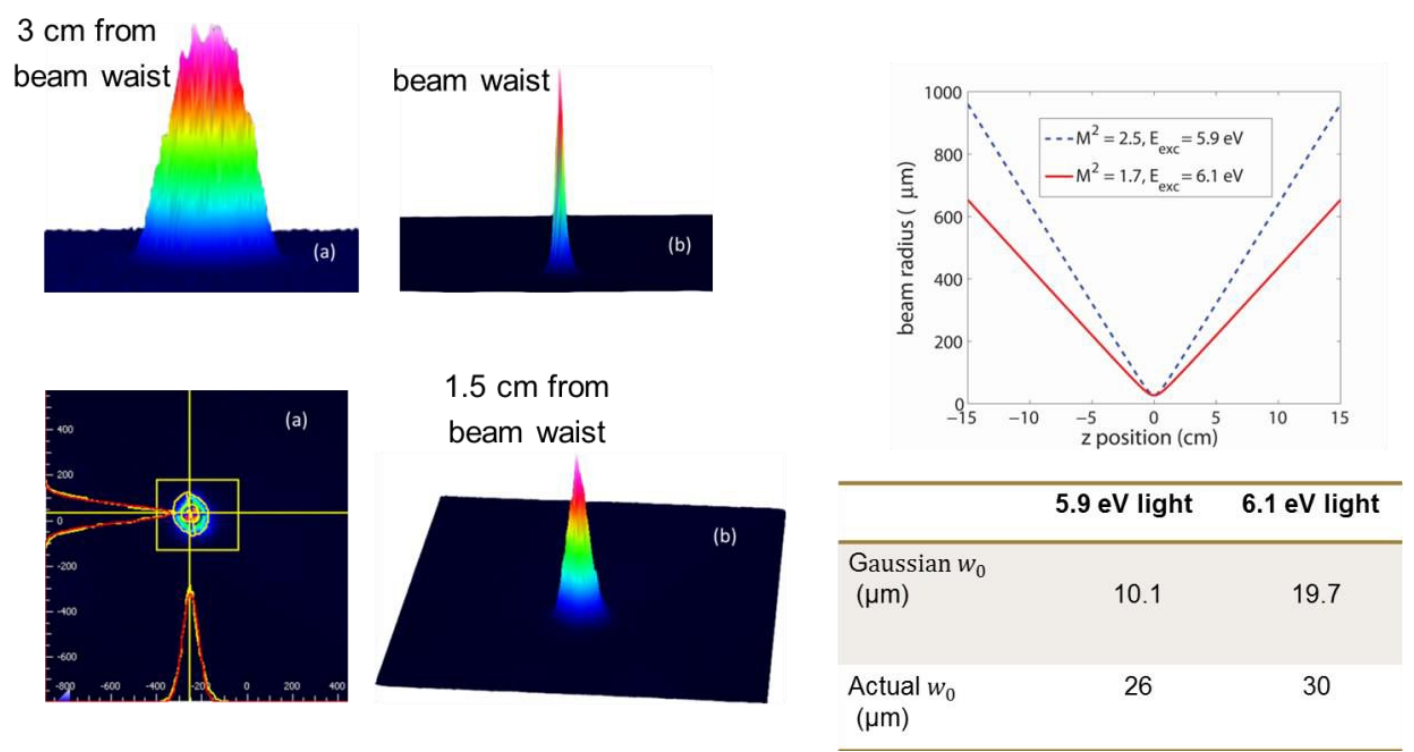

Figure 2. Measurements of the $4^{\text {th }}$ harmonic ultraviolet pulse transverse profiles are shown for several distances, $\mathrm{z}$, between the beam waist and the entrance face of the sample. The data versus $\mathrm{z}$ are fit to the $\mathrm{M}^{2}$ profile of a non-ideal Gaussian, which changes with alignment of system components for different wavelengths of the $4^{\text {th }}$ harmonic pulse.

\section{RESULTS AND DISCUSSION}

\subsection{BGO and $\mathrm{SrI}_{2} ; \eta_{e h}$}

The raw data of the PDR experiment are shown in the left panel of Fig. 3 for two materials, bismuth germanate $\left(\mathrm{Bi}_{4} \mathrm{Ge}_{3} \mathrm{O}_{12}, \mathrm{BGO}\right)$ and strontium iodide $\left(\mathrm{SrI}_{2}\right)$. The characteristic dip at the beam waist position (highest fluence versus lens position) suggests why we have called this method "interband z scan."[9] The middle panel shows that by taking into account the beam profile, sample reflectivity, and laser pulse energy, the fluence on the central axis just inside the sample surface can be associated with each z position. The fluence scale is placed across the top of the middle panel in Fig. 3. With further knowledge of the interband absorption coefficient $\alpha\left(\mathrm{cm}^{-1}\right)$, we can convert fluence to excitation density $n_{0}\left(\mathrm{eh} / \mathrm{cm}^{3}\right)$ on the central axis, just inside the sample surface. The conversion formula is shown in Fig. 3, where $F_{0}$ is the laser fluence $\left(\mathrm{eV} / \mathrm{cm}^{2}\right)$ and $h v$ is the laser photon energy $(\mathrm{eV})$. In the right panel, the light yield is plotted versus a logarithmic scale of excitation density $n_{0}$. The left and right sides $(\mathrm{z}<0$ and $\mathrm{z}>0$ ) of the $\mathrm{z}$ scan plot (middle panel) are averaged when replotting in the standard PDR format (right panel).

Inspection of the right-hand panel in Fig. 3 comparing PDR of BGO and $\mathrm{SrI}_{2}$ reveals that the roll-off versus excitation density appears quite different in the two materials. In $\mathrm{SrI}_{2}$, the light yield stays flat for a much longer expanse of excitation density $n_{0}$ before plummeting at the end when $n_{0}$ is very high. The plummet occurs at excitation densities above $10^{20} \mathrm{eh} / \mathrm{cm}^{3}$, which is typically found only at the ends of electron tracks. Thus in $\mathrm{SrI}_{2}$, the experimental light yield is almost flat and at its maximum value for all but track-end values of excitation density. Most of the energy of high energy gamma rays is deposited at lower $n_{0}$ than $10^{20} \mathrm{eh} / \mathrm{cm}^{3}$. Therefore, by direct comparison in Fig. 3, SrI 2 should have better proportionality and higher light yield than BGO, reasoning simply from the characteristic shapes of the photon density response (PDR) curves. This is indeed confirmed by their performance as scintillators. We shall see below that the shape of the $\mathrm{SrI}_{2}$ PDR fits $3^{\text {rd }}$ order quenching but not $2^{\text {nd }}$, and the shape of the BGO PDR fits $2^{\text {nd }}$ order quenching but not $3^{\text {rd }}$. So a prediction of relative proportionality and light yield can already be made just on the basis of whether the nonlinear quenching kinetics is $2^{\text {nd }}$ or $3^{\text {rd }}$ order. 
vs. position

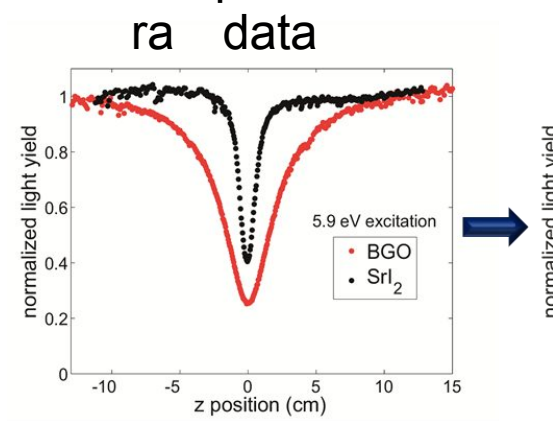

vs. Fluence

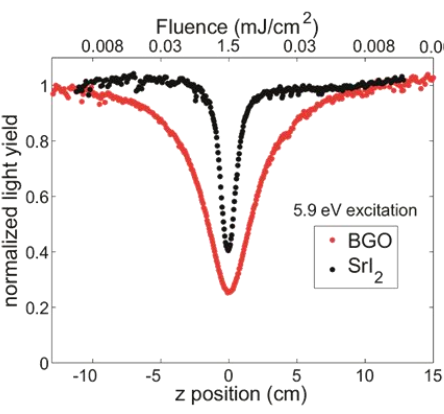

from measured

beam profile,

reflectivity, and vs. Density

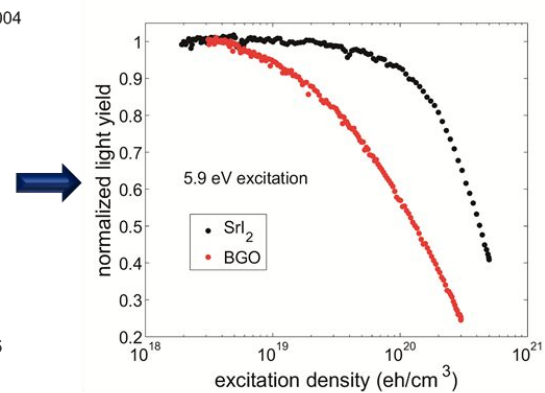

$n_{0}=\frac{F_{0} \alpha}{h v}$

laser power

Figure 3. Normalized light yield for BGO (red points, lower curve) and $\mathrm{SrI}_{2}$ (black points, upper curve) is plotted against three related variables: lens position z (left panel), laser pulse fluence $F$ (middle), and (log) excitation density $n_{0}$ (right).

Figure 4 displays the PDR data for $\mathrm{BGO}$ and $\mathrm{SrI}_{2}$ separately, with two different attempts at fitting to rate equation models of PDR also shown. The solid line in each panel is the best fit or attempted fit with a model of $2^{\text {nd }}$ order (dipole-dipole) quenching, and the dashed line is the best fit or attempted fit to a model of $3^{\text {rd }}$ order (free-carrier Auger) quenching. The conclusion is unambiguous in each case, and is summarized in terms of experimentally determined free carrier fraction $\eta_{e h}$ in the box below each plot. The excitations during nonlinear quenching in BGO are apparently electron-hole pairs (excitons) interacting as dipole radiators and receivers in the near field, whereas in $\mathrm{SrI}_{2}$ during nonlinear quenching the dominant population is free carriers $\left(\eta_{e h}=1\right)$ based on the finding of $3^{\text {rd }}$ order quenching in the PDR data.
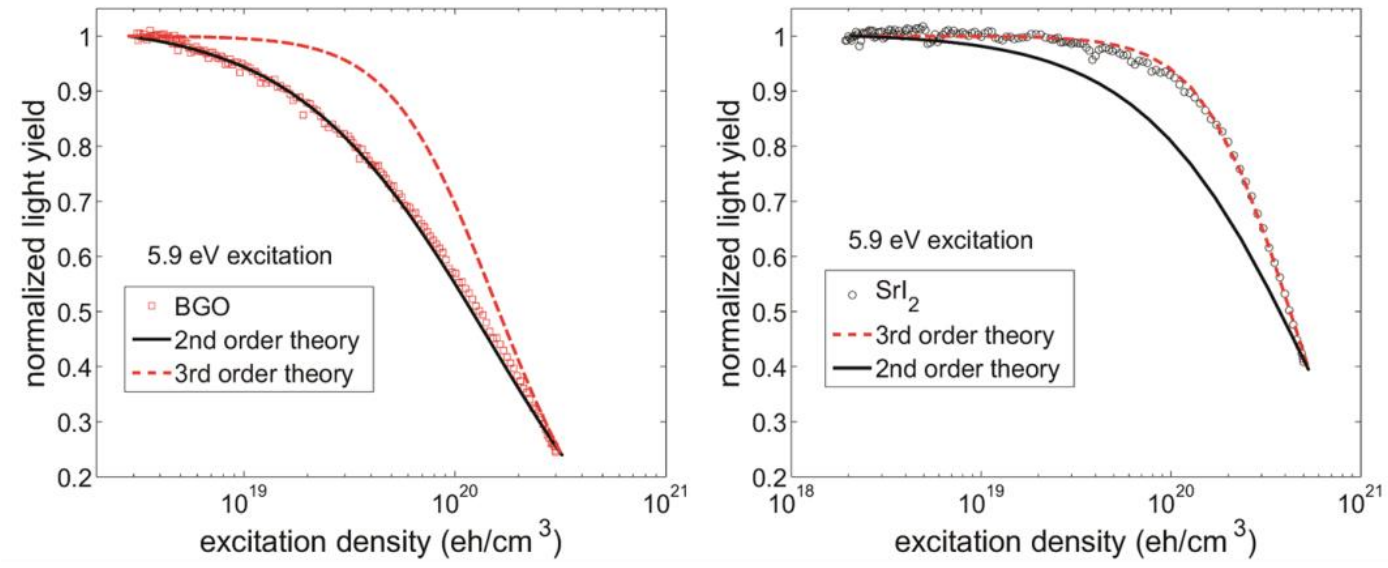

$$
\begin{gathered}
\eta_{e h} \approx 0 \\
\omega_{\mathrm{LO}}=15.3 \times 10^{13} \mathrm{~s}^{-1} \\
\tau_{\text {therm }} \leq 0.5 \mathrm{ps}
\end{gathered}
$$

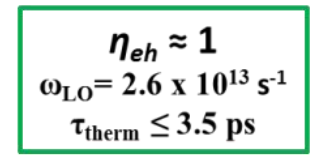

Figure 4. PDR data (open circles) for BGO on the left and $\mathrm{SrI}_{2}$ on the right. The solid line in each panel is the best fit or attempted fit with a model of $2^{\text {nd }}$ order (dipole-dipole) quenching, and the dashed line is the best fit or attempted fit to a model of $3^{\text {rd }}$ order (free-carrier Auger) quenching. The fits are unambiguous and from this, the values of $\eta_{e h}$ in each case have been deduced. 


\subsection{Rate equations and light yield models for PDR}

The $2^{\text {nd }}$ order model can be stated quite simply. It is based on the rate equation for exciton population, $N$ (excitons $/ \mathrm{cm}^{3}$ ), decaying by $1^{\text {st }}$ order radiative decay (rate constant $R_{l}$ ), linear quenching (rate constant $K_{l}$ ), and $2^{\text {nd }}$ order dipole-dipole quenching (rate constant $K_{2}$ ):

$$
\frac{d N}{d t}=-\left(R_{1}+K_{1}\right) N-K_{2}(t) N^{2}
$$

The solved population $\mathrm{N}(\mathrm{t})$ is substituted into the light yield equation:

$$
L Y=\frac{\int_{0}^{\infty} R_{1} N(t) d t d^{3} r}{\int_{0}^{\infty}\left[\left(R_{1}+K_{1}\right) N(t)+K_{2}(t) N^{2}(t)\right] d t d^{3} r}
$$

The result is the solid line fitted or attempted in Fig. 4.

The $3^{\text {rd }}$ order model takes some additional discussion as given below and in Ref. [9]. It is based on the following approximate $3^{\text {rd }}$ order rate equation

$$
\frac{d n}{d t}=-K_{1 e} n-B n^{2}-K_{3} n^{3}
$$

where $n$ is the free carrier density. In a pure material or at local excitation density much higher than that of dopants and defects, bandgap excitation leads to $n_{e}=n_{h}=n$. The term $K_{l e} n$ is the trapping rate on deep defects, $B n^{2}$ is the bimolecular pairing rate of electrons and holes to form excitons, and $K_{3} n^{3}$ is the Auger quenching rate. It is more correct to write separate coupled rate equations for electron and hole density and write the $2^{\text {nd }}$ and $3^{\text {rd }}$ order terms proportional to $n_{e} n_{h}$ and $\left(n_{e} n_{h} n_{e}+n_{e} n_{h} n_{h}\right)$ respectively. The more exact expressions of the bimolecular and Auger rates in coupled rate equations for electrons and holes are in fact used in our data fitting to account for effects of charge separation should it occur, but the form of the simplified Eq. (3) is useful for discussion of concepts in this document.

\section{3 $\eta_{e h}\left(T_{e}, t\right)$; born excitons; thermalization and capture of initially hot electrons}

We have just seen that $\eta_{e h}$, defined as the average or effective value of $\eta_{e h}\left(T_{e}, t\right)$ during nonlinear quenching, can be measured by PDR experiments. The free carrier fraction $\eta_{e h}\left(T_{e}, t\right)$ is an evolving quantity as electrons and holes cool from the nonequilibrium energies of their creation and begin to associate (while also diffusing) as excitons. This raises the question, what was the "born" free carrier fraction $\eta_{e h}(t=0)$ ? For insight, we look at calculations by Vasil'ev on the excitations created by scattering of high energy electrons in $\mathrm{BaF}_{2}$, including distributions at extremely short time after excitation, down to 0.04 fs.[13]

The calculations provide two ways of looking at the exciton fraction at the instant of excitation. In the left panel of Fig. 5 , the simulated dielectric function $\varepsilon_{2}$ in light grey line, partial electron-hole contribution $\varepsilon_{2}{ }^{e h}$ neglecting exciton contributions (grey shading), and energy loss function $\operatorname{Im}(-1 / \varepsilon)$ in dark line with exciton structure are plotted for $\mathrm{BaF}_{2}$ as functions of photon energy.[13] The ratio of exciton and free-carrier final states directly excited by inelastic scattering of the incident electron is given by the partial contributions of these two types of excitations in the energy loss function. Visual separation of those two contributions is best indicated by comparison of $\varepsilon_{2}$ and $\varepsilon_{2}{ }^{e h}$ as a function of energy. Integration over photon energy spanning the energy deposition of a stopping primary electron suggests that excitons comprise only about $1 \%$ of direct excitations by electrons with initial energy greater than $1 \mathrm{keV}$. The fraction should decrease with rising initial electron energy.

In the right panel, simulated energy distribution and particle number distribution among electrons, holes, and excitons excited in $\mathrm{BaF}_{2}$ by a $1 \mathrm{keV}$ electron, evaluated at $0.04 \mathrm{fs}$ after electron passage at the local position is shown.[13] By integrating the number of valence excitons and the number of electrons or holes in the bar chart representations, we conclude that about $2 \%$ of the excitations are bound valence excitons at $0.4 \mathrm{fs}$ after the electron passage at the local position. Core excitons are not counted because they will decay predominantly to energetic electrons and holes, and they do not represent much oscillator strength in any case. 

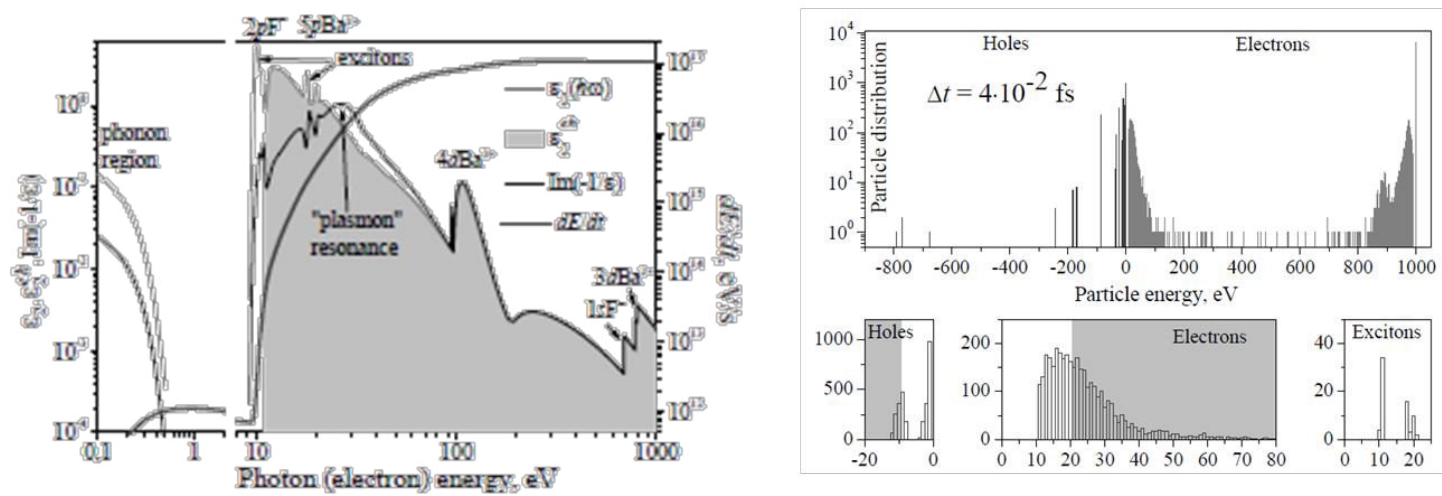

Figure 5. Imaginary part of the dielectric function $\varepsilon_{2}$ in light grey line, partial electron-hole contribution $\varepsilon_{2}^{e h}$ neglecting exciton contribution (grey shading), and energy loss function $\operatorname{Im}(-1 / \varepsilon)$ in dark line with exciton structure are plotted for $\mathrm{BaF}_{2}$ in the left panel as functions of photon energy by A. N. Vasil'ev.[13] The right panel shows simulated energy distribution and particle number distribution among electrons, holes, and excitons excited in $\mathrm{BaF}_{2}$ by a $1 \mathrm{keV}$ electron, evaluated at $0.04 \mathrm{fs}$ after electron passage at the local position. Figures used with permission of $\mathrm{A}$. N.

Vasil'ev.[13]

Based on the example of $\mathrm{BaF}_{2}$, about $2 \%$ of excitations by a high energy electron go directly into bound exciton states. The rest are free carriers with some excess kinetic energy to be dissipated before possibly pairing. The electron and hole will tend to move apart according to the initial relative velocities of their wave packets $\left(\boldsymbol{v}_{g e}-\boldsymbol{v}_{g h}\right)$ based on the local slope of the electron energy bands, while all the time losing energy. As Vasil'ev et al have calculated [7,14], geminate pairs are created if the electron thermalizes within the Onsager radius of the hole it left at creation. Other excitons can continue to form over time, between non-geminate carrier pairs. Exciton formation can be delayed if electron thermalization is slow, as in crystals with low optical phonon frequency. Our hypothesis is that the ratio of exciton fraction and free-carrier fraction during nonlinear quenching depends on the electron thermalization rate, which in turn depends on the optical phonon frequency $\omega_{L O}$.

The relation of electron thermalization time to capture on holes (making excitons) is illustrated in Fig. 6 showing calculations of electron energy relaxation and the fraction of electrons captured on holes as a function of time in CsI and YAP (yttrium aluminum perovskite, $\mathrm{YAlO}_{3}$ ) by $\mathrm{Li}$ et al [15]

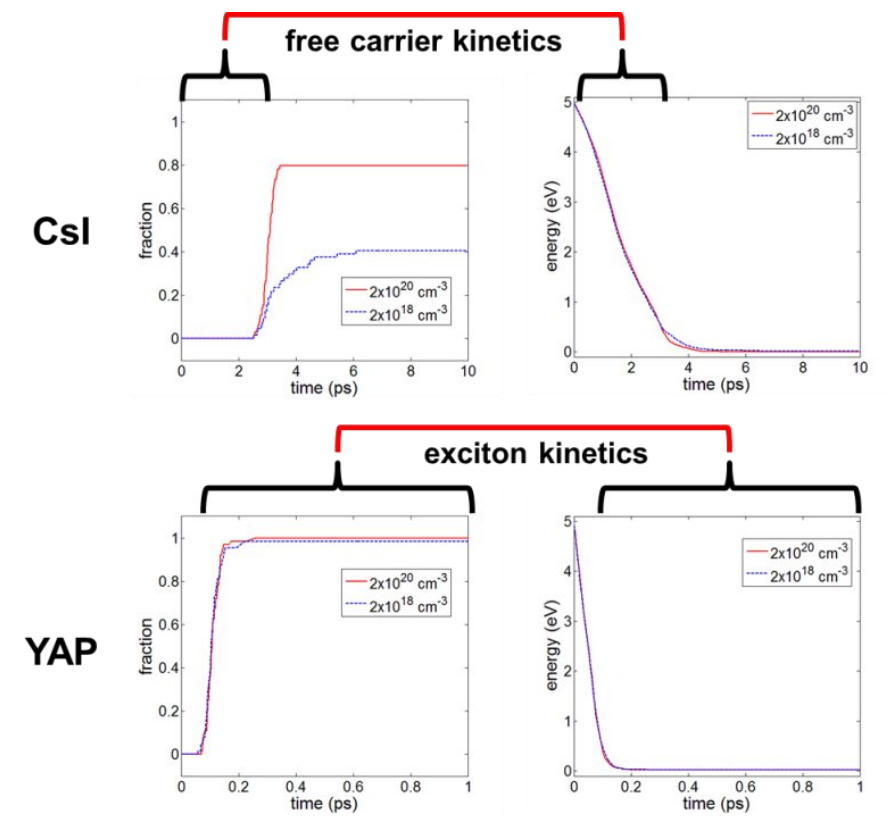

Figure 6. Monte Carlo simulations of the thermalization of hot electrons and capture on holes in CsI and YAP calculated by Li et al.[15] The method is similar to that employed by Wang et al [4,5] and Vasilev et al. [7,14] including calculation of phonon emission rate. The electron is captured when it scatters to a bound state in the electron hole potential that is at least $3 \mathrm{kT}$ below the ionization limit. Results are shown for initial excitation densities of $2 \quad 10^{20} \mathrm{~cm}^{-3}$ (red) and $2 \quad 10^{18} \mathrm{~cm}^{-3}$ (blue). 
The calculation method employs Monte Carlo simulation with phonon scattering rates calculated by methods similar to Wang et al[4,5] and Vasil'ev et al. [7,14] The electron is captured by a hole when it scatters by phonon emission to a bound state in the electron-hole potential that is at least $3 \mathrm{kT}$ below the ionization limit.[15] Notice that the time axes for the YAP and CsI plots are a factor of 10 different. It is seen that electron-hole pairing in CsI is prevented until electron thermalization is nearly complete on a scale of about $3 \mathrm{ps}$, and then capture proceeds rapidly after that delay. The same happens in the simulations for YAP, except about 30 times faster. Cooling and capture in YAP is substantially subpicosecond, i.e. around $100 \mathrm{fs}$. In order to match the experimental finding of pure $3^{\text {rd }}$ order quenching in CsI, nonlinear quenching should be mostly complete in 3 ps or less. We have labeled the time intervals in which free-carrier kinetics would apply and in which exciton kinetics would apply, for both materials in Fig. 6.

An experimental observation relevant to this topic comes from picosecond infrared absorption spectroscopy of pure and Tl-doped CsI at room temperature, shown in Fig. 7.[16] The upper and lower panels are for different kinetic energies shared by the electron and hole, $2 h v-E_{g a p}=0.1 \mathrm{eV}$ and $3.06 \mathrm{eV}$ respectively. The initial spike of absorption nearly coincident with the excitation pulse has a very broad spectrum and may be attributed to free carrier absorption. It can be seen that when the initial excitation is $3.06 \mathrm{eV}$ above the band gap, the free carrier spike is larger relative to the more slowly developing self-trapped exciton absorption. It is relatively smaller for excitation just slightly above the band gap. The rapid drop in free carrier absorption could be due to shallow capture of electrons in high Rydberg states around holes, transferring oscillator strength dominantly to the deep infrared spectrum out of our experimental spectral range, and to self trapping of holes which could transfer hole oscillator strength to the ultraviolet. The re-growth of absorption having a spectrum previously attributed to self-trapped excitons in CsI at room temperature [17] could represent relaxation of self-trapped excitons to their metastable radiative state. The time for that process is seen in Fig. 7 to be about 10 ps, roughly consistent with the calculated electron-hole capture rate for CsI in Fig. 6.

Free-carrier Auger quenching rather than exciton-exciton dipole quenching should apply in the initial time period when the (hot) carriers are free. When the carriers are thermalized and captured on spatially separated traps, their contribution to Auger quenching should effectively terminate. This would be the likely case in CsI:Tl. Holes tend to self-trap right where they are in the host lattice, while electrons should trap mainly on $\mathrm{Tl}^{+}$, present at $0.1 \%$ levels. The main point is that electrons and holes do not generally trap at the same location in scintillators like CsI:Tl, so trapping in a doped crystal can effectively terminate Auger recombination. When the electrons are captured on self-trapped holes to form STE, the dominant quenching term from that sub-population should be 2nd order dipole-dipole, but by then their concentration has been reduced by diffusion and Auger quenching, so they represent only a fraction of the initial electron population. Thus their contribution to quenching can be small compared to the free-carrier contribution in the earlier, denser conditions.
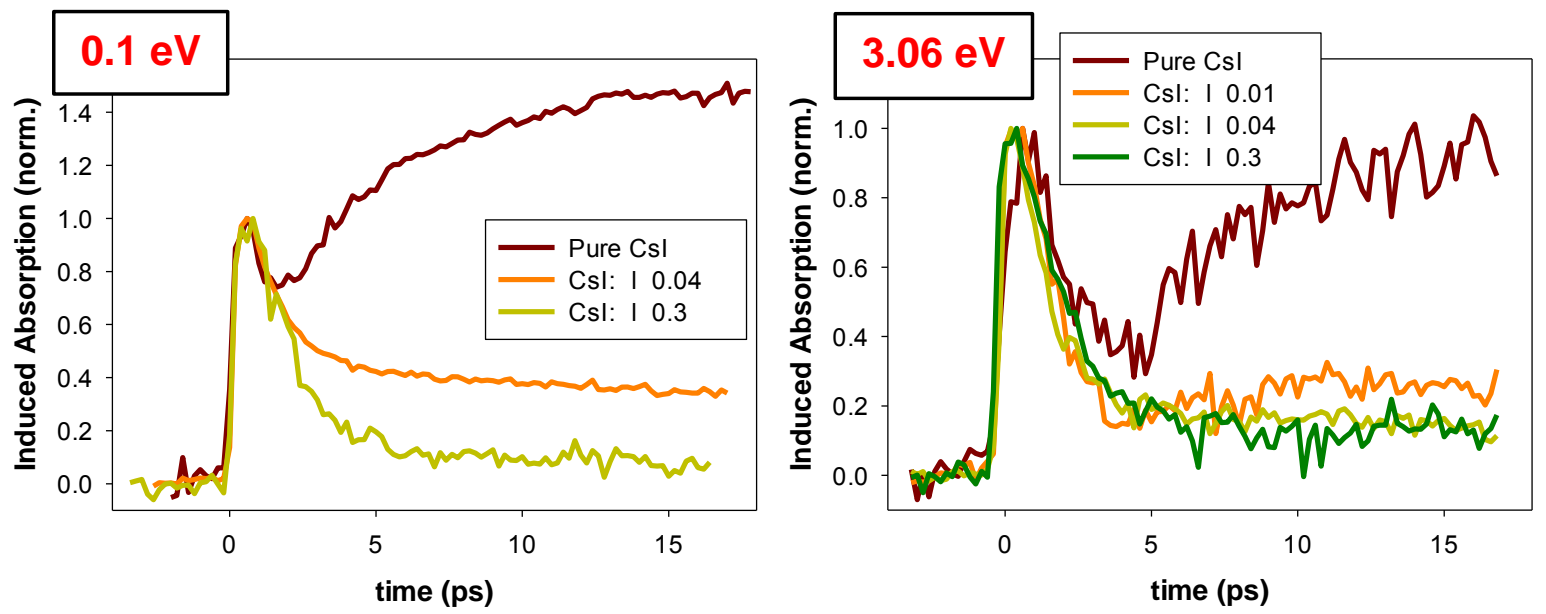

Figure 7. Picosecond infrared absorption spectroscopy of pure and Tl-doped CsI at room temperature. The left and right panels are for different kinetic energies shared by the electron and hole, $2 h v-E_{g a p}=0.1 \mathrm{eV}$ and $3.06 \mathrm{eV}$ respectively. 


\subsection{Mixed kinetic order in NaI and Kinetic Monte Carlo simulations of PDR}

$\mathrm{NaI}$ is the exception that proves the aforementioned rule, in view of its higher $\omega_{L O}$ relative to most other iodide scintillators. Whereas nonlinear quenching is pure $3^{\text {rd }}$ order in $\mathrm{SrI}_{2}$ and CsI, we observe in PDR of NaI that there is a mixture of $2^{\text {nd }}$ order and $3^{\text {rd }}$ order kinetics, depending on how far above the exciton bands the PDR laser excitation is tuned.

The mixed order NaI results in Fig. 8 (left) were excited with $6.1 \mathrm{eV}$ laser photons, which produce hot carriers with up to $0.3 \mathrm{eV}$ excess energy. When the experiments were done with $5.9 \mathrm{eV}$ laser photons only about $0.1 \mathrm{eV}$ above the exciton ionization limit, the PDR can be fit by pure $2^{\text {nd }}$ order quenching, as shown in Fig. 8(right) and Fig. 9. Superimposed in Fig. 9 is the Kinetic Monte Carlo (KMC) simulation of $2^{\text {nd }}$ order quenching[18,19], fitted to the laser PDR data[9]. As mentioned in the Introduction, the PDR experiment is measured without the complex track structure and associated gradients driving diffusion that occur in gamma-ray or electron excited luminescence. This allows KMC fitting of laser PDR to calibrate rate parameters used in the simulation, without having to deal with the track structure complications. This is a valuable tool in conjunction with anticipated KMC fitting of EER data including Compton coincidence (e.g.

SLYNCI) and K-dip.

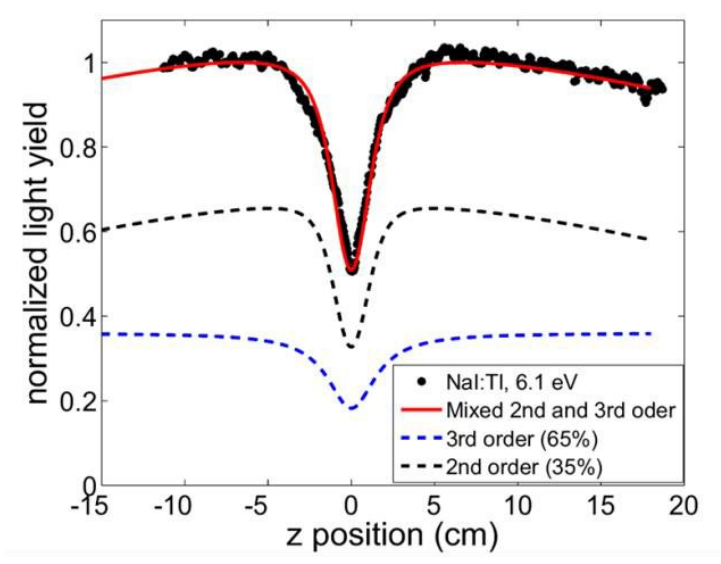

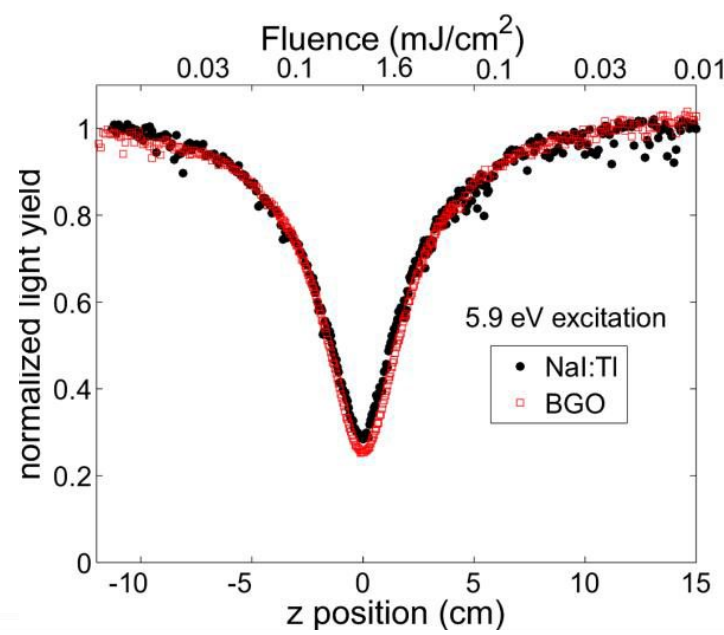

Figure 8 Photon density response of NaI:Tl excited at $6.1 \mathrm{eV}$ and $5.9 \mathrm{eV}$. For $6.1 \mathrm{eV}$ excitation, the fit corresponds to a mixed population of excited states during nonlinear quenching: $65 \%$ free carriers and $35 \%$ excitons. For $5.9 \mathrm{eV}$ excitation, the $2^{\text {nd }}$ order model provides a good fit, implying that the excitations are excitons. BGO data with known pure $2^{\text {nd }}$ order kinetics is superimposed for comparison.

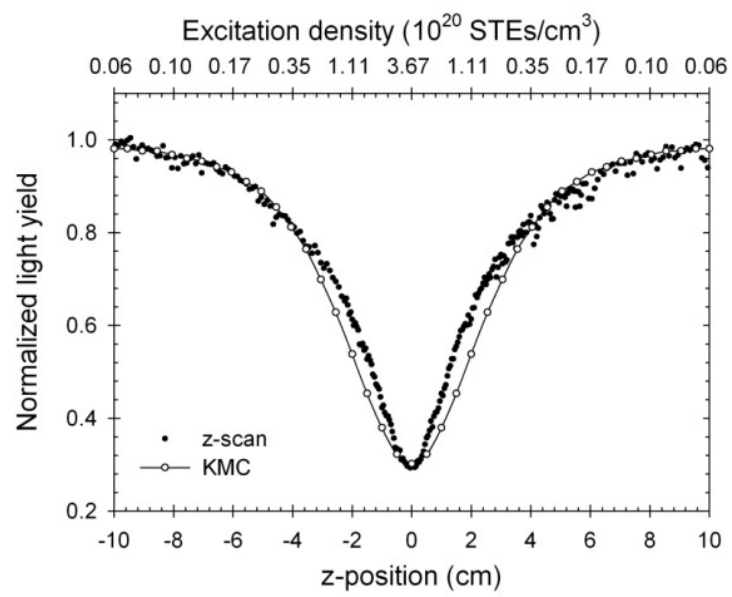

Figure 9. Comparison of the calculated light yield by Kinetic Monte Carlo method (open circles) $[18,19]$ with experimental PDR results (solid circles) [9] for $\mathrm{NaI}(0.1 \% \mathrm{Tl})$ excited by $5.9-\mathrm{eV}$ photons as a function of the position of the beam waist (z-position) and excitation density. 


\subsection{Hot free-carrier kinetics and associated light yield model}

We have been showing $3^{\text {rd }}$ order fits of the PDR data in Figs. 4 and 8 without yet stating exactly what the $3^{\text {rd }}$ order model is. The delay in discussing it is tied up with the phenomenon illustrated in Fig. 6, that slow electron thermalization can introduce a delay in exciton formation. We will build the model for $3^{\text {rd }}$ order quenching in view of finite electron thermalization time in stages, starting from the conventional model that neglects this factor. The basic rate equation that includes $3^{\text {rd }}$ order quenching was given previously as Eq. (3). The conventional assumption is that all of the rate terms compete on the same time scales. We will refer to this as the "conventional $B n^{2}$ model", so named because the $2^{\text {nd }}$ order exciton formation term is responsible ultimately for light emission.

Following an assumption made by Murray and Meyer [20], we consider for the moment that only the free carriers forming excitons (rate $\mathrm{Bn}^{2}$ ) will have a chance ultimately to contribute to scintillation light, and so write the following proportionality to the corresponding light yield

$$
L Y \propto \frac{\int_{0}^{\infty} B n^{2} d t d^{3} r}{\int_{0}^{\infty}\left(K_{1 e} n+B n^{2}+K_{3} n^{3}\right) d t d^{3} r}
$$

where the rate constants $K_{l e}, B$, and $K_{3}$ were defined in Eq. (3).

Putting aside the $3^{\text {rd }}$ order term for the moment, we note that Murray and Meyer considered only the $1^{\text {st }}$ and $2^{\text {nd }}$ order terms without the indicated time integrals in Eq. (4) and then divided out the common factor of $B n^{2}$, obtaining

$$
L Y \propto\left(\frac{K_{1 e}}{B n}+1\right)^{-1}
$$

for the trend of rising light yield with excitation density. They later remarked on the ultimate decreasing light yield expected at very high excitation density, which we include by restoring the Auger term and writing an expression in all 3 orders, similar to Eq. (5). This qualitatively predicts a hump in light yield versus excitation density $n$ :

$$
L Y \propto\left(\frac{K_{1 e}}{B n}+1+\frac{K_{3} n}{B}\right)^{-1}
$$

It is worth noting that in going from the conventional light yield expression in Eq. (4) to the simplified trend expressed in Eqs. (5) and (6), a step was taken that does not strictly apply to pulsed excitation such as in scintillator operation. Dividing out the $B n^{2}$ rate term can only be done mathematically in a "continuous excitation" experiment where the excited population is continuously resupplied. Then the light yield is proportional simply to the ratio of radiative and total rates of depleting the excited population, as represented in Eqs. (5) and (6). (We also note that J. B. Birks [21] made a similar continuous excitation assumption in deducing the "Birks term" as it has been called by various workers in the scintillator field.) But the real application of scintillators, the measurements of electron energy response, and the photon density response laser experiment all detect the integrated light yield after pulsed excitation. This requires doing the time integrations shown explicitly in Eqs (2) and (4), before forming the ratio. The "continuous excitation model" can be convenient for discussing qualitative trends, but for fitting quantitative data the integrals must be done first and then the ratio formed to predict light yield.

For large carrier density $n$, where both photon density response and electron energy response experiments generally show a roll-off of light yield due to nonlinear quenching, Eq. (6) predicts an inverse first power dependence $(1 / n)$ of Light Yield versus $n$. Figure 10 plots the prediction of Eq. (4) including proper integration, labeled as the "conventional $B n^{2}$ model", in comparison to measured photon density response of $\mathrm{SrI}_{2}$. The plot of the 2 nd order quenching model represented by Eq. (2) is also shown, and neither can fit the data because they fall off too slowly. In fact, the conventional $B n^{2}$ model falls off even more slowly than the $2^{\text {nd }}$ order exciton model, Eq. (2). 


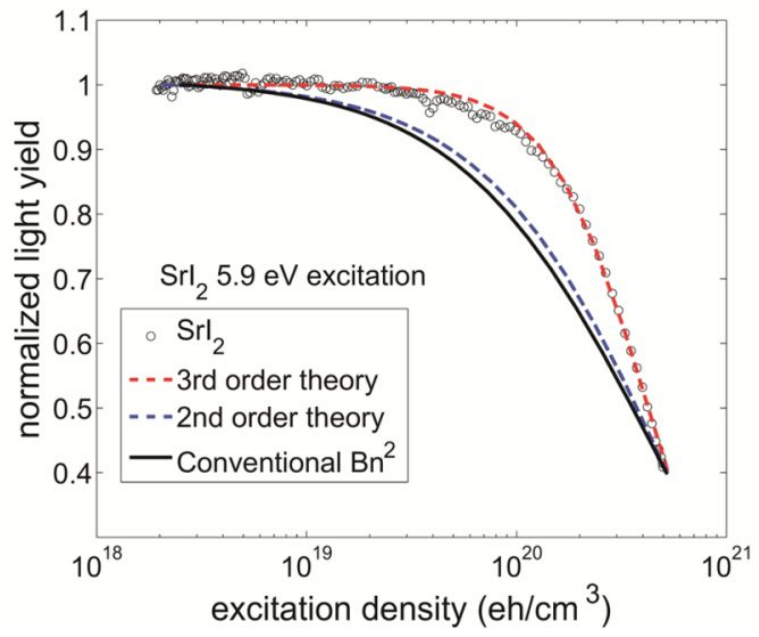

Figure 10. PDR data in $\mathrm{SrI}_{2}$ (open circles) is shown along with three model attempts to fit the data. The red dashed curve that fits the experimental data uses the $3^{\text {rd }}$ order model to be developed in the next few paragraphs. The blue dashed curve that cannot be made to fit is the $2^{\text {nd }}$ order model described by Eq. (2). The solid curve that also fails to fit the data is the "conventional $B n^{2}$ " light yield, Eq. (4), based on the conventional $3^{\text {rd }}$ order rate equation, Eq. (3), in which all terms compete on the same time scale.

The failure of Eq. (4) to fit the roll-off slope in a 3rd order quenching material is one of several experimental results leading us to conclude that the first, second, and third order kinetic terms do not all compete on the same time scale in materials like $\mathrm{SrI}_{2}$ and the alkali iodides, where hot carriers persist during a significant part of the time for nonlinear quenching. The very fact of finding pure 3rd order quenching in photon density response for $\mathrm{SrI}_{2}$ and CsI already led us to invoke hot electrons as the physical phenomenon holding off exciton formation and other electron capture channels for a finite time in iodides. The hot electron thermalization time is approximately inversely proportional to optical phonon period $[4,5,7,14]$ and can be as long as $7 \mathrm{ps}$ in CsI.[4,5] There seems almost no other way of explaining the occurrence of pure 3rd order quenching in the iodides having low $\omega_{L O}$ while the oxides with high $\omega_{L O}$ exhibit pure 2nd order quenching.

The logical extension of this explanation is that not all the rate terms in Eqs. (3) and (4) can be competing in the same time range. Electron capture on traps or dopants $(A n)$ or on holes $\left(B n^{2}\right)$ is strongly suppressed until the electrons have thermalized. This was illustrated by the calculations of electron temperature and of the paired fraction of electrons and holes versus time comparing CsI and YAP in Fig. 6. Therefore, we want to see if the slope of the roll-off of photon density response in $\mathrm{SrI}_{2}$ and other iodides can be properly fit when we take the thermalization time dependence of each kinetic order into account in the free-carrier rate equation (Eq. 3) and the expression for light yield (Eq. 4). Use of explicitly calculated rate "constants" dependent on time via electron temperature is being pursued in current Monte Carlo simulations of these processes.[15] However, for a simple analysis of the photon density response data that at least takes electron thermalization into account, we have assumed a step-wise time dependence in each kinetic order of the freecarrier rate equation [9] as follows:

$$
\frac{d n}{d t}=-A n \Theta\left(t-\tau_{t h}\right)-B n^{2} \Theta\left(t-\tau_{t h}\right)-K_{3} n^{3} \Theta\left(\tau_{t h}-t\right)
$$

where $\Theta\left(t-\tau_{t h}\right)$ is the Heaviside step function turning on after $\tau_{t h}$, and $\Theta\left(\tau_{t h}-t\right)$ turns off after $\tau_{t h}$.

In this way, we assert that the coupling rates into exciton formation $\left(B n^{2}\right)$ and carrier trapping $(A n)$ cannot turn on until it becomes possible to trap electrons on holes or on dopants, i.e. until the electron has thermalized to within the trapping potential depth. (See Fig. 6) Li et al are working to calculate the thermalization time as a function of electron energies excited in the photon density response experiments.[15] However for the time being, we assume that the trapping and bimolecular exciton formation channels turn on after a hot electron thermalization time approximated as $6 \mathrm{ps}$ in iodide crystals. This estimate of $6 \mathrm{ps}$ is chosen as representative of the 7 ps maximum thermalization time in CsI [4] and the 6 ps capture time of electrons on $\mathrm{Tl}^{+}$in $\mathrm{CsI}: \mathrm{Tl}(0.3 \%)$ [17]. The free-carrier Auger recombination is similarly modeled as 
turning off when the carriers thermalize and trap on spatially separated sites (in CsI:Tl and similar activated scintillators, especially halides).

Based on the time-step rate equation (7), we can proceed to write the expression for light yield taking hot electron thermalization into account. The integration of Eq. (7) has been done numerically for fitting, but is not directly expressible in analytic form. However, the following expression separating the terms of Eq. (7) acting on different time scales into a product of probabilities of survival from each successive stage yields fits practically indistinguishable from the numerical integration of Eq. (7). It has the advantage of being conceptually easy to dissect into three physical stages:

$$
L Y \approx\left(1-\frac{\int_{0}^{\tau_{\text {therm }}} \int_{V} K_{3} n(\vec{r}, t) d t d^{3} r}{\int_{V} n(\vec{r}, t=0) d^{3} r}\right) \frac{\int_{\tau_{\text {therm }}}^{\infty} \int_{V} B n(\vec{r}, t)^{2} d t d^{3} r}{\int_{\tau_{\text {therm }}}^{\infty} \int_{V}\left(K_{1} n+B n^{2}\right) d t d^{3} r}\left[\frac{\int_{\tau_{\text {therm }}}^{\infty} \int_{V} R_{1} N(\vec{r}, t) d t d^{3} r}{\int_{\tau_{\text {therm }}}^{\infty} \int_{V}\left(K_{1, \text { exc }} N+R_{1} N+K_{2} N^{2}\right) d t d^{3} r}\right]
$$

A crucial feature of Eq. (8) to note is that the $3^{\text {rd }}$ order quenching process is limited to the left-hand bracket governing hot carrier quenching and determining the survival fraction that passes on to the middle bracket of processes such as capture of thermalized carriers on deep traps $\left(K_{l} n\right)$ and on holes $\left(B n^{2}\right)$. The left-hand bracket as a multiplier imposes a steep slope on the roll-off of light yield at high $n$, in agreement with the PDR experiments. The time integrals in the middle term run formally from $\tau_{t h}$ out to $\infty$, but the rates within are all sufficiently fast that the trapping and exciton formation can be virtually complete in tens of picoseconds. The survivors of the middle bracket are excitons, passed on to the right-hand bracket as the exciton density $N$. The quadratic nature of electron-hole recombination is represented in the exciton formation (middle bracket), but the only radiative rate in the entire multiplied string of brackets is that of generalized (free or trapped) exciton radiative decay $R_{l} N$ in the right-hand bracket.

In a more complete treatment to be published [22], it is shown that the free-carrier rate equation Eq. (3) should account separately for the rates of deep trapping $K_{l} n$ and shallow trapping $S_{l} n$. If the shallow traps thermally release stored charge within the scintillation time window, the $S_{l} n$ rate can also contribute to eventual formation of trapped or free excitons and thus becomes another kinetic term in the light yield.

\subsection{Auger rate constants}

By fitting Eq. (8) to PDR data, we obtain the rate constants $K_{2}$ and/or $K_{3}$ appropriate to the populations of excitations, as well as $\eta_{e h}$ specifying the fractional populations. The values of $K_{3}$ measured from these PDR (Z scan) experiments in $\mathrm{SrI}_{2}$, CsI, NaI, and CdTe are compared in the following Table and graph to $K_{3}$ measured by other methods in materials that are primarily semiconductors of interest for light-emitting diodes and lasers. The empirical "band-gap rule" of Auger rate constants predicts that $K_{3}$ decreases as the

\begin{tabular}{lcccc}
\hline \hline Crystal & $\begin{array}{c}K_{3}\left(\mathrm{~cm}^{6} \mathrm{~s}^{-1}\right) \\
\times 10^{-29}\end{array}$ & Reference & $E_{g}(\mathrm{eV})$ & $\begin{array}{c}\omega_{\mathrm{LO}}\left(\mathrm{s}^{-1}\right) \\
\times 10^{13}\end{array}$ \\
\hline $\mathrm{InSb}$ & 1200 & {$[36]$} & 0.17 & 3.7 \\
$\mathrm{GaSb}$ & 7 & {$[36]$} & 0.7 & 4.3 \\
$\mathrm{GaAs}$ & 0.7 & {$[37]$} & 1.43 & 5 \\
$\mathrm{CdTe}$ & 0.5 & PDR & 1.44 & 3.1 \\
$\mathrm{In}_{0.15} \mathrm{Ga}_{0.85} \mathrm{~N}$ & 0.2 & {$[38]$} & 2.64 & 14 \\
$\mathrm{In}_{0.09} \mathrm{Ga}_{0.91} \mathrm{~N}$ & 0.14 & {$[38]$} & 2.83 & 14 \\
$\mathrm{SrI}{ }_{2}$ & 0.7 & PDR & 5.5 & 2.6 \\
$\mathrm{NaI}: \mathrm{Tl}$ & 3.2 & PDR & 5.7 & 3.47 \\
$\mathrm{CsI}: \mathrm{Tl}$ & 6.6 & PDR & 5.8 & 1.79 \\
$\mathrm{CsI}$ & 4.5 & PDR & 5.8 & 1.79 \\
\hline \hline
\end{tabular}

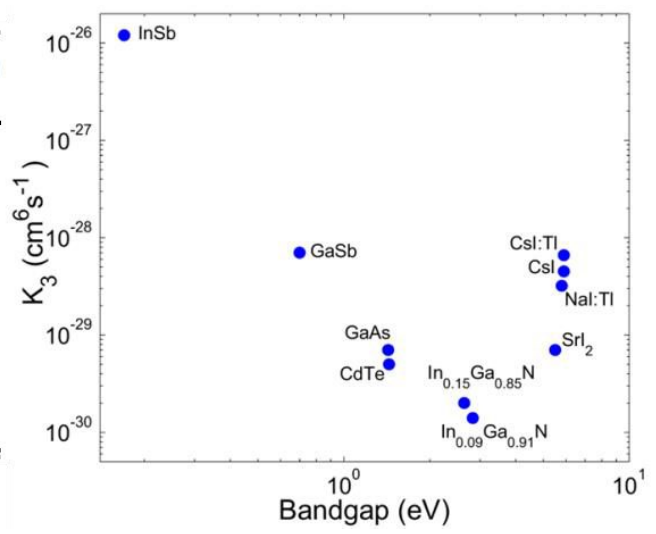

Figure 11. Auger recombination rate constant $K_{3}\left(\mathrm{~cm}^{6} \mathrm{~s}^{-1}\right)$ is tabulated and plotted versus bandgap for CdTe, $\mathrm{SrI}_{2}$, NaI, and CsI measured by laser PDR in this work, alongside values from the literature on several semiconductors.

band gap increases. The basic reason for this is that the energy given to the spectator electron or hole by e-h recombination increases with band gap. The corresponding final-state wavevector of the spectator particle increases accordingly, soon exceeding the range of wavevector $\boldsymbol{k}$ in the initial state and thus progressively closes down the 
possibility of satisfying $\boldsymbol{k}$ conservation. Our measured $K_{3}$ for CdTe falls in line with the other semiconductors, but $K_{3}$ for the iodides with larger band gap lies considerably above the trend extrapolated from the semiconductors.

There are at least two interpretations for the Auger rate constants found in alkali iodides from PDR measurements being higher than predicted by the band-gap rule extrapolated from semiconductors: (1) The hole in alkali halides[23] (and alkaline earth halides [23] including $\mathrm{SrI}_{2}$ [24] is self-trapped. This localization to one lattice site removes the wavevector conservation rule that otherwise severely limits possible final states of the Auger transition as band gap increases. If at least one of the interacting carriers is localized (e.g. self-trapped), the initial state already contains a spread of $\mathrm{k}$ components, so the Auger rate should not be so restricted. If we feel confident to press details of the data, we can point out that the Auger rate constant of $\mathrm{SrI}_{2}$ is about 6x smaller than the average of CsI and NaI. Since we have seen that $\mathrm{SrI}_{2}$ quenches only by Auger recombination, the fact that it has a smaller Auger rate constant than alkali iodides seems to be yet another reason why $\mathrm{SrI}_{2}$ has better proportionality and light yield. As a possible cause for the difference, one wonders if possibly the hole is not as completely localized in $\mathrm{SrI}_{2}$ as in the alkali iodides. (2) The electron is hot during the free carrier phase when nonlinear quenching is assumed to occur. The carriers excited by laser in the photon density experiments remain out of equilibrium with the lattice, possessing up to $300 \mathrm{meV}$ excess kinetic energy (up to $2300 \mathrm{~K}$ electron temperature) in alkali iodides and up to $1.6 \mathrm{eV}$ in $\mathrm{SrI}_{2}$ during the Auger recombination observed in the $6.1 \mathrm{eV}$ PDR experiments. [9] It is known that lattice temperature accelerates Auger recombination particularly in wide-gap materials, for the reason discussed just above. At high lattice temperature, phonons can provide the needed momentum in indirect Auger events, as is well known. Similarly, excess electron temperature should also increase the probability of conserving momentum for large energy transfers in Auger recombination. Although moderately hot electrons do not possess mean momenta as large as phonons, neither do hot carriers as sources of momentum imply a 4th participant (phonon) as required in indirect Auger processes. Both reasons support plausibility of the enhanced Auger rates observed in iodide crystals by the PDR experiment.

Yet another alternate interpretation could be that the premise of Eq. (7) and the surrounding discussion is wrong, i.e. that the free-carrier Auger (3rd order) quenching continues well beyond the time limit imposed by carrier cooling and trapping. Then a smaller $K_{3}$ rate constant would be deduced from the photon density response. But then we would have to look elsewhere than hot electron thermalization to resolve the observed $3^{\text {rd }}$ order/ $2^{\text {nd }}$ order dichotomy of iodides vs oxides and to account for changing kinetic order upon tuning photon energy about $200 \mathrm{meV}$ above the band gap in iodides. We have not found an alternative explanation for the latter observations.

\subsection{Adding electron tracks and predicting electron energy response}

We have used laser PDR data measured without the complicating spatial gradients of electron tracks to determine values of $\eta_{e h}, K_{2}$, and $K_{3}$. To compare the predictions based on these and other parameters to electron energy response of scintillators, one needs to put the track gradient, diffusion, and excitation density distribution back into the picture. We do this by measuring track radius near the track end and constructing a light yield model that includes carrier diffusion and the linear \& nonlinear rate constants to obtain a predicted local light yield as a function of excitation density, $Y_{L}\left(n_{0}\right)$. Then the distribution of excitation densities from slowing of a high-energy electron of initial energy $E_{i}$ is calculated, e.g. using Geant 4 , to yield the distribution of excitation densities, $F_{e h}\left(n_{0}, E_{i}\right)$. Convolution of these two functions of $n_{0}$ yields the electron energy response $Y_{e}\left(E_{i}\right)$ for comparison to Compton-coincidence and K-dip experiments. [25] The procedures from diffusion, quenching, thermalization, and local light yield up through the final convolution have been developed in Refs. [1,2,4-8,25-29]. A brief overview will be given here, beginning with effects of diffusion and nonlinear quenching in electron tracks.

Refs.[26-28] developed a local light yield model based on the premise that by diluting excitation density and in some cases separating charges in the track core, diffusion of electrons and holes can affect the nonlinear quenching. Refs. [11,26-28] and related works set out to evaluate the dominant trends in light yield resulting from a model corresponding to the rate Eqs. (1) and (2). It was found that the dominant diffusion effects manifest themselves differently in different classes of materials. We will illustrate in turn two competitions in which diffusion can preserve some excitations from the nonlinear quenching that would otherwise occur. The first competition is simple dilution of carrier pair density by ambipolar diffusion of electrons and holes to diminish the amount of dipole-dipole quenching that would otherwise occur in the dense track core if carriers and excitons were static. The essence of the track-dilution effect of ambipolar diffusion on nonlinear quenching is illustrated for the full exciton rate equation, i.e. Eq. (1) with diffusion included, 
applied to an assumed cylindrical electron track in Fig. 12. It is simply a competition in whether excitons will more quickly reduce their concentration $N$ by the quenching itself (a loss of the exciton number) or by diffusion to larger radius causing reduction of exciton density and thus of quenching rate, but not necessarily a reduction of exciton number.

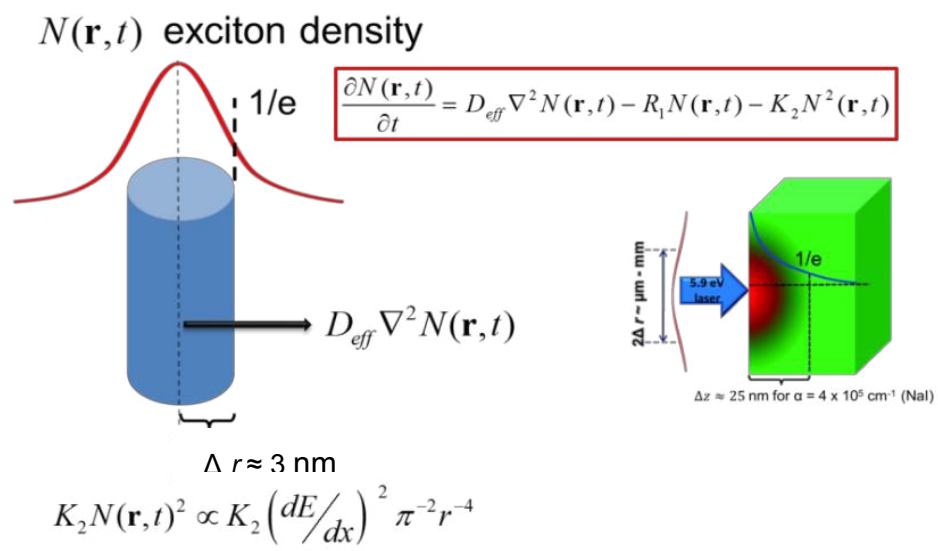

Figure 12. The illustration on the left represents the competition between loss of luminescence due to $2^{\text {nd }}$ order quenching in an electron track, and rescue by diffusion out of the densely populated track core. The illustration on the right compares the weaker gradients in laser interband excitation for PDR.

A physical phenomenon affecting halides more than oxides and semiconductors is spatial charge separation when the electron and hole mobilities are widely unequal. This is especially pronounced when there is self-trapping of holes. The effect is to protect the electron-hole pair from early nonlinear quenching, which requires that electron and hole distributions are spatially overlapped. If, as in alkali halides, the electron mobility is much greater than the hole mobility, the electrons will outrun the holes in the radial concentration gradient of the modeled cylindrical track, trapping separately as independent carriers in preference to pairing as excitons, up to the time of eventual de-trapping and recombination on activators or defects. $[26,29]$ The excitations that are captured as excitons on an activator will emit promptly. In contrast, the excitations that are captured as separated electrons and holes on different activators or defects must recombine more slowly by de-trapping mechanisms. During that process, the fraction of independently trapped carriers $(I F)$ will be exposed to deep trapping, particularly by charged defects having a large cross section for independent charge carriers. $I F$ in general changes along the track length due to changing electric field effects that accompany the changing excitation density. The trapping hazard is represented by the multiplier of fraction $I F$, called the linear quenched free-carrier fraction $k_{1} \cdot[26,27]$ The survival fraction is thus $\left(1-k_{1} I F\right)$. Plotting $\left(1-k_{1} I F\right)$ versus onaxis excitation density $n_{0}$ for several different hole mobilities $\mu_{h}$ relative to fixed $\mu_{e}$ shows a rising trend versus $n_{0}$.

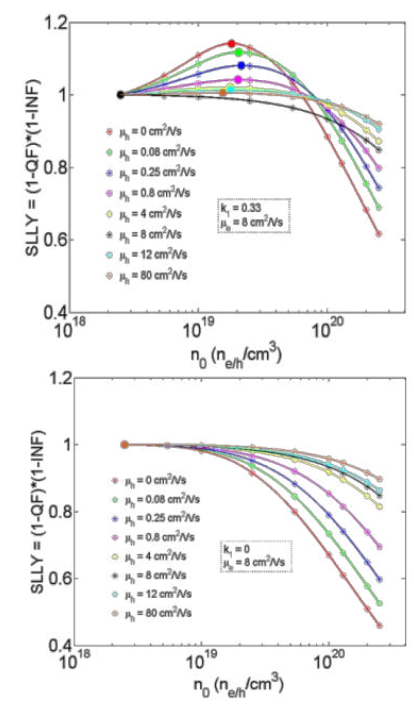

$$
k_{1}=\frac{\int_{0}^{\infty} K_{1 e} n d t}{\int_{0}^{\infty}\left(K_{1 e} n+B n^{2}+K_{3} n^{3}\right) d t}
$$

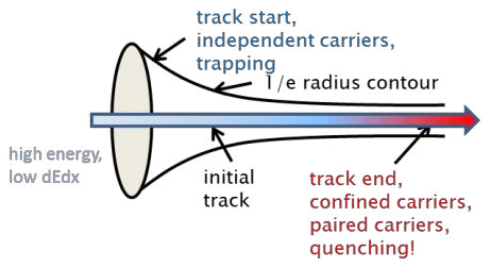

Figure 13. Simulated local light yield $Y_{L}\left(n_{0}\right)=(1-Q F)\left(1-\mathrm{k}_{1} I F\right)$ versus on-axis excitation density $n_{0}$ is plotted for different hole mobilities as listed. The electron mobility is fixed at $8 \mathrm{~cm}^{2} / \mathrm{Vs}$. The linear quenched fraction $k_{l}$ is 0.33 in the upper frame and 0 in the lower frame. 
In some ways analogous to the product of "Birks and Onsager" terms in the model described by Payne et al [1,2], the product $(1-Q F)\left(1-k_{1} I F\right)$ approximates the overall probability of an electron-hole pair surviving nonlinear quenching $Q F$ in the track core, followed by the probability of being driven to independent status by unequal diffusion and yet surviving deep trapping to recombine radiatively. Figure 13 plots the simulated local light yield in the model of Refs. [26-28] for a series of hole mobilities $\mu_{h}$ while holding $\mu_{e}=8 \mathrm{~cm}^{2} / \mathrm{Vs}$. Without a significant mismatch of $\mu_{e}$ and $\mu_{h}$, there is no hump. But also without some linear quenching $k_{l}$, there is no hump either.

\subsection{Track radius}

The track radius determines $n_{0}$ corresponding to a given linear energy deposition rate $d E / d x$ and also determines the gradient that promotes radial carrier diffusion. Track radius is thus a crucial parameter of the model, both its initial value at the time of energy deposition, and its evolving value under diffusion of hot and thermalized electrons. For that matter, there is a radius of the hole distribution and a radius of the electron distribution. Depending on whether holes self-trap and how much electrons or holes diffuse, charge separation can build up. This has consequences for both radiative and nonradiative recombination on different time scales. Recent experimental [9] and computational [30] work has deduced probable values for the radius of the nonlinear quenching zone [9] and the radius of the self-trapped hole distribution[30,31] near the track end in $\mathrm{NaI}$ and more recently in $\mathrm{SrI}_{2}$ [32]. The values are all in agreement on approximately $3 \mathrm{~nm}$ radius. The data and method for the experimental determination are illustrated in Fig. 14 and have been discussed in more detail in Ref. [9].

The laser fluence in a PDR (z-scan) experiment can be adjusted to produce the same nonlinear quenching at the bottom of the dip as is seen in K-dip spectroscopy at a track-end value of electron energy (e.g. $80 \mathrm{eV}$ ) in NaI:Tl. Briefly, K-dip spectroscopy analyzes the light yield attributable to excitation by K-shell photoelectrons of specific energy selected by tuning synchrotron radiation relative to the $\mathrm{K}$ edge. [3] It is assumed that the same excitation density $n_{0}$ will produce the same amount of nonlinear quenching in each of the two experiments compared in Fig. 14. We equate the peak densities $n_{0}$ expressed in Eqs. (9) and (10) and solve for $r_{N L Q}$, the effective radius in which nonlinear quenching occurs:

$$
\begin{aligned}
& n_{0}=\frac{d E / d x}{\beta E_{g a p} \pi r_{N L Q}^{2}} \\
& n_{0}=\frac{F_{0} \alpha}{h v} \\
& r_{N L Q}^{2}=\frac{h v d E / d x}{F_{0} \alpha \beta E_{g a p} \pi}
\end{aligned}
$$

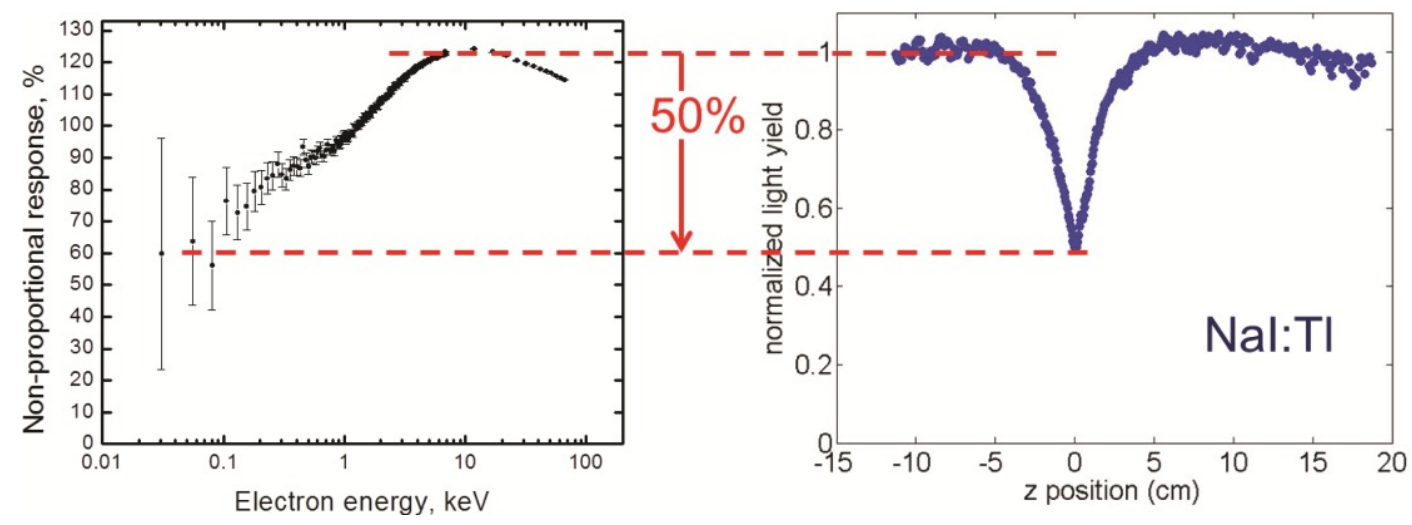

Figure 14. K-dip spectroscopy [3] for NaI:Tl (left) with 50\% quenching near the track end. On the right are the z-scan results for NaI:Tl with the uv laser fluence tuned to produce the same level of quenching from Ref. [9]. 
The result in NaI:Tl with $\alpha \approx 4 \times 10^{5} \mathrm{eh} / \mathrm{cm}^{3}$, ,[33] $F_{0}=0.4 \mathrm{~mJ} / \mathrm{cm}^{2}, h v=5.9 \mathrm{eV}, d E / d x($ at $80 \mathrm{eV})=64 \mathrm{eV} / \mathrm{nm}[31], \beta=$ 2.5 [34], and $E_{\text {gap }}=5.8 \mathrm{eV}$ is $r_{N L Q} \approx 3 \mathrm{~nm}$ near the track end. Using the NWEGRIM Monte Carlo code, Gao et al have calculated the radius of the self-trapped hole distribution at track end in NaI to be $2.8 \mathrm{~nm}[30]$, as shown in Fig. 15 and discussed briefly below.

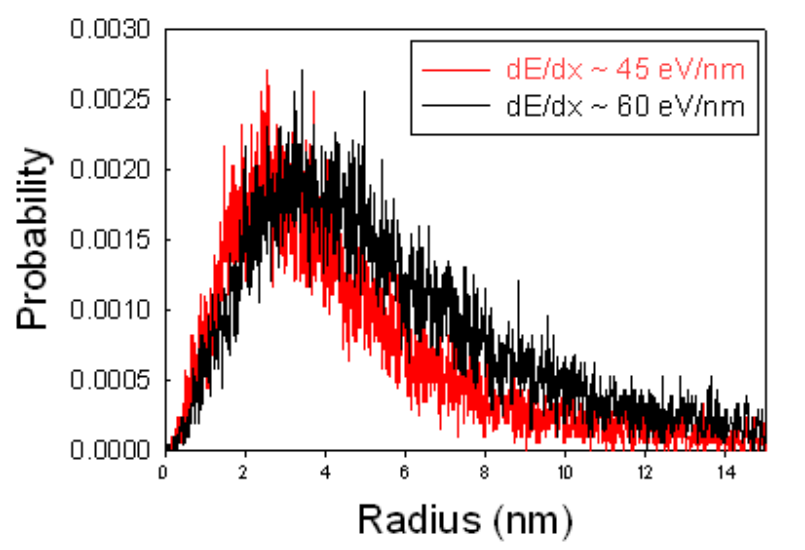

Figure. 15 Calculated probability distribution of electrons at end of the electron cascade (effectively before diffusion and thermalization) near the track end in NaI. This is assumed to approximate the distribution of self-trapped holes.

Although a single value of track radius can be evaluated in the z-scan experiment, its fluctuation cannot be easily determined in experimental measurements, which may be important for the further development of an accurate model of scintillator proportionality. The NorthWest Electron and Gamma Ray Interaction with Matter (NWEGRIM) code,[30] has been developed to simulate the response of scintillator materials, evaluating their intrinsic properties, stopping power, and maximum theoretical light yield. It has been employed to determine the electron distribution in $\mathrm{NaI}$ at initial points of creation, i.e. at the end of the electron cascade terminating with the start of phonon cooling at $\mathrm{E}_{\mathrm{cbm}}+\mathrm{E}_{\text {gap }}$. This is effectively before diffusion and thermalization. It is likely that the static self-trapped hole distribution in an alkali halide crystal such as $\mathrm{NaI}$ is the same as this initial electron distribution radius. As shown in Fig. 15, the peak position of the radial distribution at the track end in $\mathrm{NaI}$ is $2.8 \mathrm{~nm}$, and the distribution ranges from 0.1 to $14 \mathrm{~nm}$. This self-trapped hole distribution is in reasonable agreement with that measured by the z-scan experiments in NaI, discussed above. It is also consistent with an earlier estimate by Vasil'ev et al.[31]

\subsection{Hot electrons}

Carrier pairs are created by slowing of the primary electron, with electron and hole kinetic energies distributed over a wide range. The electrons lose energy very rapidly by secondary e-h production until reaching an energy one bandgap above the conduction band minimum (cbm), and analogously for holes. From there, the cooling is slower by phonon emission (picoseconds or less for $\mathrm{LO}$ phonon processes) as equilibrium with the lattice temperature is approached. There are a number of indications that the main part of nonlinear quenching takes only a matter of picoseconds, as does free-carrier diffusion toward an equilibrium distribution [26], so it can be important to consider diffusion and nonlinear quenching while the electrons are still in nonequilibrium energy states.

The extent of electron-hole pair recombination and the distances traveled by hot electrons during thermalization are illustrated in Figure 16 for four halide scintillators, namely, $\mathrm{NaI}, \mathrm{CsI}, \mathrm{CaF}_{2}$, and $\mathrm{BaF}_{2}$. These results have been obtained from a Monte Carlo model of electron thermalization [4,5] that incorporates electron scattering with optical and acoustic phonons and also includes the effects of internal electric fields. The model uses as input the spatial and kinetic energy distributions of electron-hole pairs at the end of the energy cascade, as calculated by NWEGRIM. The kinetic energy distributions of hot electrons at the end of the energy cascade extend up to the band gap energy. Therefore, the majority of the electrons have high kinetic energies relative to the thermal energy. As a result, the distributions of the distances 
traveled by the electrons that are able to escape the electric field of the STHs and eventually reach thermal energy (referred to as "stopped" electrons) peak between approximately 20 and $60 \mathrm{~nm}$ and can extend up to a few hundreds of nanometers (Figure 16 - right). Although the thermalization distances can be large, a significant fraction of the electrons (referred to as "recombined" electrons) are not able to escape the electric field of the STHs and recombine with STHs to form STEs (Figure 16 - left). The increase in stopping power at low incident $\gamma$-ray energies generates high electron-hole pair densities, which leads to an increased probability for electron-hole pair recombination. This, in turn, should result in a greater extent of second and/or third-order nonlinear quenching at low incident $\gamma$-ray energies. Second order quenching is now treated in the KMC model, with inclusion of third order anticipated at a future time. The MC model predicts that the four materials yield different extents of electron-hole pair recombination due mostly to differences in their electron mean free paths, LO phonon energies, initial densities of electron-hole pairs, and static dielectric constants. In particular, the LO phonon energy is a key factor that affects electron thermalization. Indeed, the higher the LO phonon energy is, the more favored phonon emission is over phonon absorption and the more energy is transferred to the lattice for each phonon emission event. Electron-hole pair recombination occurs in the early stages of the thermalization process and those electrons that recombine with STHs travel distances of at most 10 to $20 \mathrm{~nm}$ (Figure 16 - right - inset).
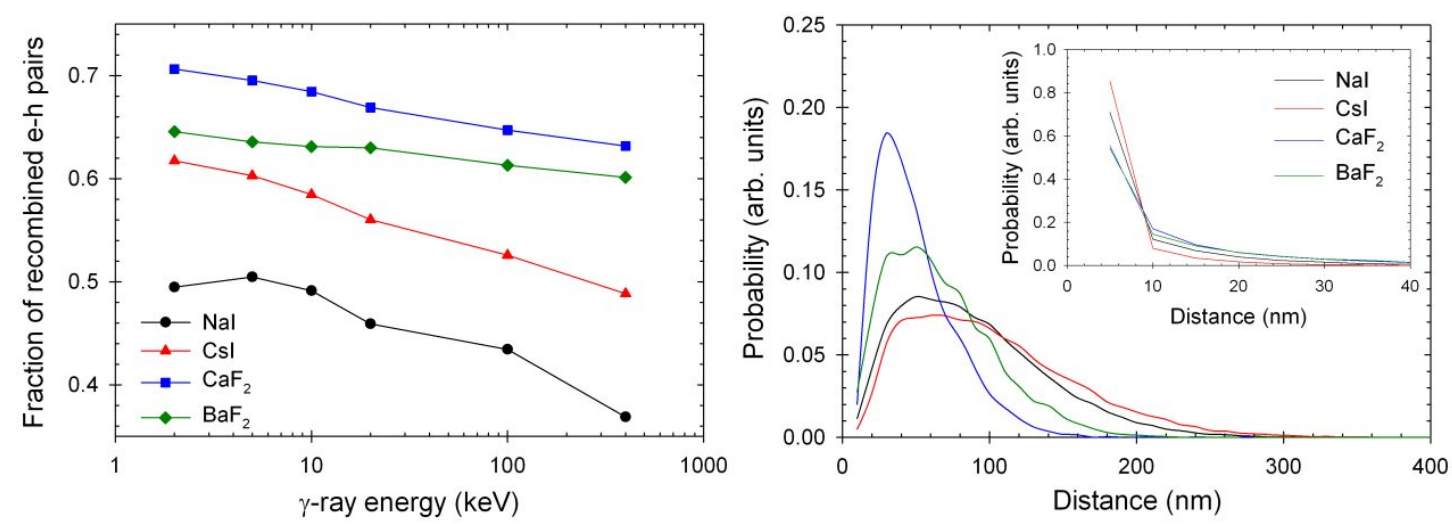

Figure 16. (left) Fraction of recombined electron-hole pairs as a function of incident $\gamma$-ray energy for two alkali iodide (NaI and CsI) and two alkaline-earth fluoride $\left(\mathrm{CaF}_{2}\right.$ and $\left.\mathrm{BaF}_{2}\right)$ scintillators. (right) Thermalization distance distributions of the "stopped" electrons for the same four scintillator materials (2-keV incident $\gamma$-ray). The inset shows the thermalization distance distributions of the "recombined" electrons from the same simulations.

It appears that the consideration of hot and thermalized diffusion can resolve some subtleties having large consequences for scintillator performance, such as why NaI:Tl and $\mathrm{SrI}_{2}: E u$ have such different proportionality and light yield despite having quite similar values of carrier mobility, nonlinear quenching rate constants, and other parameters normally considered to help determine proportionality and light yield. A clue is found from inspecting the conduction band structures through the phonon cooling range in Fig. 17.

Just as a visual impression, the conduction bands in $\mathrm{NaI}$ are comparatively sparse and not terribly far from free-electron like. In contrast, the conduction bands in $\mathrm{SrI}_{2}$ are densely packed and generally appear flatter, partly attributable to avoided crossings of some of the densely packed bands. The cause of this visual impression is basically that the hot electron group velocities proportional to slopes of the bands appear lower on average in $\mathrm{SrI}_{2}$ than in NaI. The difference in packing density of the electronic bands is attributable to the more complex unit cell in $\mathrm{SrI}_{2}$ (24 atoms) compared to $\mathrm{NaI}$ (2 atoms). Calculation of the average group velocities in these two crystals was presented in Ref. [6], and used to form a hypothesis illustrated schematically in the lower part of Fig. 17. If hot electrons diffuse farther radially in NaI than in $\mathrm{SrI}_{2}$, then the recombination of thermalized and/or shallow-trapped electrons with holes trapped near the track core will have to get through a longer path of quenching (deep-trapping) defects in $\mathrm{NaI}$ than in $\mathrm{SrI}_{2}$. This raises the linear quenched fraction $k_{l}$ in $\mathrm{NaI}$ relative to $\mathrm{SrI}_{2}$ (and also relative to other halides with complex unit cells "multivalent halides" in the characterization of Payne et al.[2]). We have already seen in Fig. 13 that increasing $k_{l}$ increases the "halide hump" in electron energy response. Proportionality suffers when the hump is large. By definition, $k_{l}$ also decreases total light yield. 


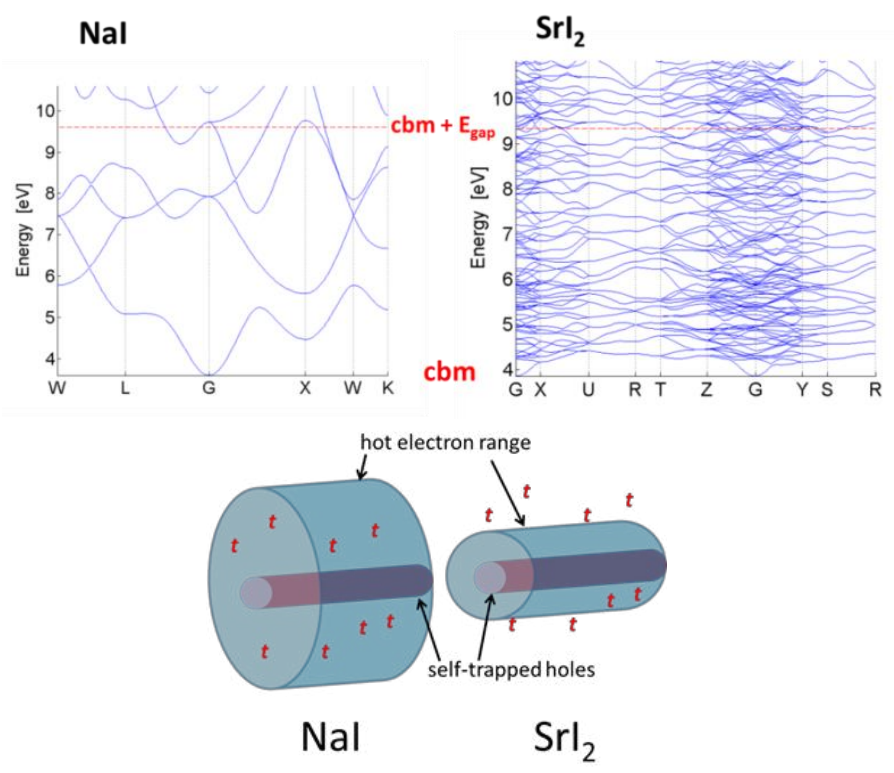

Figure 17. Upper part: Conduction band dispersion curves are compared through the range of phonon-cooling of hot electrons from $\mathrm{E}_{\mathrm{cbm}}$ up to $\mathrm{E}_{\mathrm{cbm}}+\mathrm{E}_{\mathrm{gap}}$ for $\mathrm{NaI}$ and $\mathrm{SrI}_{2}$. Lower part: Hypothesis based on faster diffusion of hot electrons in NaI compared to $\mathrm{SrI}_{2}$, leaving a track core of self-trapped holes near the center. Recombination by diffusion after thermalization encounters a longer path through the field of deep electron traps in NaI.

\section{CONCLUSIONS}

From the foregoing discussion, it should be possible to look at branch points between basic types of behavior of scintillator materials according to the value (high or low) of a few key parameters. In the following exercise, we will consider four such parameter choices in sequence as a "decision tree" to see if indeed it can predict general characteristics of proportionality and light yield based on a small number of parameters that are measureable apart from the scintillator proportionality measurement itself. If it can be confirmed, it is the beginning of a design rule for scintillator discovery and engineering.

The first parameter to be considered, therefore in some sense the most fundamental, is the (highest) LO phonon frequency. The corresponding branch point is diagrammed in Fig. 18, with experimental plots of PDR illustrating physical consequences of branching to high and low values of $\omega_{L O}$. Low $\omega_{L O}$ in the right branch means that the carriers thermalize slowly relative to the time in which the main part of nonlinear quenching occurs. The basis for this conclusion is the experimental observation of pure third order quenching kinetics in the PDF data for CsI and $\operatorname{SrI}_{2}$. Identifying $3^{\text {rd }}$ order quenching with free-carrier Auger recombination, we have concluded that in materials of the right branch, free carriers are too hot during the nonlinear quenching phase to become bound in exciton or trap states. Since the time for electron thermalization by LO phonons in iodide crystals has been calculated $[4,5,7,14]$ to be on the order of a few picoseconds, the above line of reasoning indicates that the main nonlinear quenching is complete within a few picoseconds. This conclusion is consistent with previous time-resolved data directly[25] and indirectly [17] related to nonlinear quenching in CsI. The bottom line for the decision is that free carriers dominate the nonlinear quenching process in the right branch of Fig. 18 corresponding to low $\omega_{L O}$. Most iodides qualify as low $\omega_{L O}$, although we saw in the PDR data of Fig. 8 that the light mass of sodium in NaI puts it in a category of mixed kinetic order, with free carriers and excitons apparently coexisting during nonlinear quenching. Heavy metal bromides and chlorides have not yet been run in the laser PDR experiments because of their larger bandgaps, but we tentatively lump them in the right branch based on $\omega_{L O}$ as the class of "heavier halides", especially when the halide is paired with a heavy metal. As a graphic illustration with real data, Fig. 18 includes PDR of $\mathrm{SrI}_{2}$ as a paradigm of the materials classified in the right branch.

The left branch is for high $\omega_{L O}$, characteristic of oxides and fluorides. High $\omega_{L O}$ causes rapid carrier thermalization. The PDR experiments on the oxides ( $\mathrm{BGO}$ and $\mathrm{CdWO}_{4}$ ) that have been measured exhibited pure $2^{\text {nd }}$ order quenching, 
consistent with dipole-dipole annihilation. This indicates that excitons in some electronic state had formed before the main part of nonlinear quenching. BGO is shown as the example of a PDR curve characterizing materials in the left branch. The PDR comparison in the bottom center of Fig. 18, already discussed in Figs. 3 and 4, emphasizes the point that both proportionality and light yield are already affected in an important way by this first material branch point, controlled by the value of $\omega_{L O}$. The winner in both proportionality and light yield at this point is the right branch simply because of the flatter and more advantageous $3^{\text {rd }}$ order PDR curve. Indeed, the known scintillators with combined highest light yield and best proportionality, such as $\mathrm{SrI}_{2}: \mathrm{Eu}, \mathrm{BaBrI}: \mathrm{Eu}$, and $\mathrm{LaBr}_{3}: \mathrm{Ce}$ should fall in the right branch, according to $\omega_{L O}$. So do some more modestly-performing scintillators like NaI:Tl, CsI:Tl, but we have not yet considered the second-level branching.

\section{Scintillator physical "Decision Tree"}

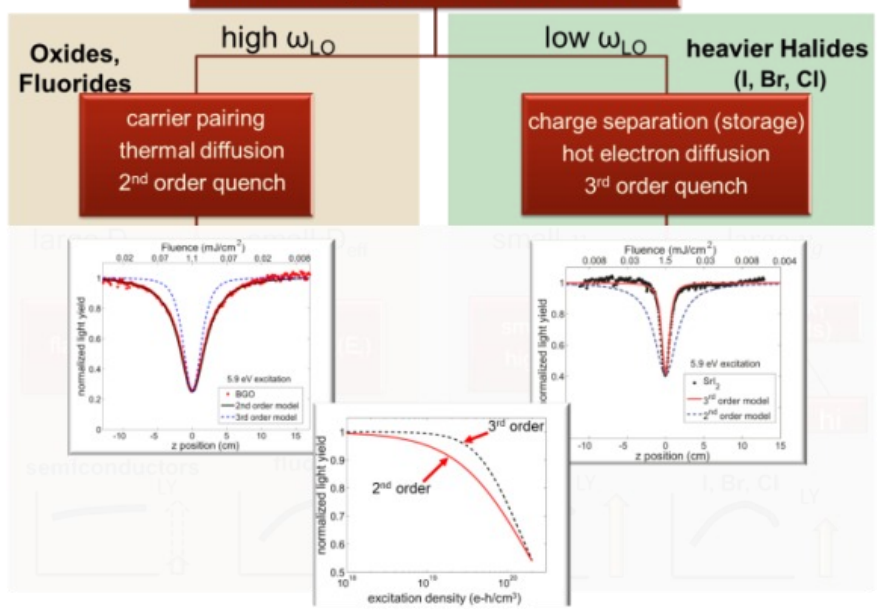

Figure 18. Top level branching between exciton and thermalized ambipolar diffusing pairs on the left and hot free carriers on the right, according to high or low LO phonon frequency.

Materials classified in the left branch have carriers that are thermalized (at least within one LO phonon energy of kT), and possibly paired as some bound state of electron and hole, before the main part of nonlinear quenching. Then as suggested in the discussion of Fig. 12, it is reasonable to describe effects of diffusion around electron tracks in terms of ambipolar diffusion (coefficient $D_{\text {eff }}$ ) of thermalized carriers. This becomes the second-level branching parameter on the left side - large or small $D_{\text {eff. }}$. Large $D_{\text {eff }}$ includes semiconductors used for luminescence (such as ZnSe:Te), and those used for charge collection detectors (high purity Ge, CdTe, CZT) which we include in the present survey by letting energy-dependent charge collection (Q) stand in for energy-dependent light yield (LY). YAP:Ce is also in the branch of large $D_{\text {eff }}$ because the near equality of electron and hole effective mass (Setyawan et al mass ratio $m_{r}=1.2$ [35]) yields large $D_{\text {eff }}$ relative to other oxide crystals.[27] Large $D_{\text {eff }}$ allows carriers to escape the nonlinear quenching zone in the track core and thus gives a relatively flat electron energy response curve which is illustrated schematically at the lower left. Because of diffusing to low excitation density, the electron-hole radiative recombination suffers relative to defect trapping and so the light yield (LY) of semiconductor scintillator detectors, and YAP:Ce as well, is modest to low. Charge collection $(\mathrm{Q})$ in semiconductors can be promoted by applied electric field and low defect concentration. Diffusion, albeit in different directions for electrons and holes in an applied electric field, is a good thing for charge collection. Characteristic schematic predictions of Q and LY in this class are indicated by the bar chart at lower left.

The second-level right branch toward small $D_{\text {eff }}$ applies to most oxides other than YAP:Ce, where higher hole mass begins to weigh on the ambipolar diffusion coefficient. Calculated band effective masses were used indirectly to represent or estimate $D_{\text {eff }}$ or its stand-in, $m_{h} / m_{e}$, in Refs. [27] and [35]. Refractive index at the visible scintillation wavelength anticorrelates generally with band gap of the host, which in turn correlates with effective masses at both band edges. This was used in Ref. [11] to organize scintillators by refractive index in a way that amounts approximately to a stand-in for effective mass and therefore for carrier diffusion coefficients. In all three forms of experimental surveys of the nonproportionality ( $\sim$ roll-off of electron energy response) among oxide scintillators, the trend was confirmed that lower effective diffusion coefficient implies greater nonproportionality, as a broad trend. [11,27,35] 


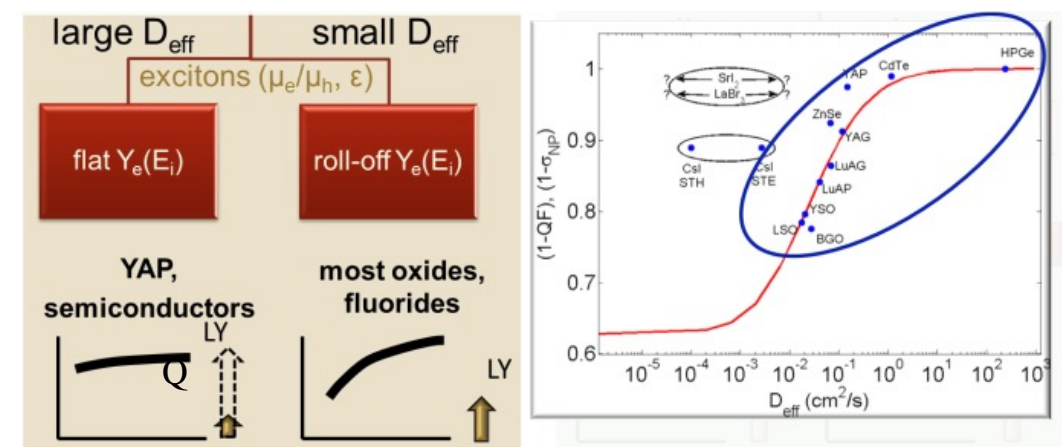

Figure 19. Second level branching of thermalized carrier pairs and excitons according to large or small effective diffusion coefficient $D_{\text {eff, }}$ correlating with schemiatic representations of electron energy response curve shape and total light yield (LY, filled arrow) or charge collection (Q, dashed arrow).

The second level branching parameter on the right hand side should, for symmetry, be the diffusion coefficient for hot electrons, "hot $D_{e}$. " We want to put it quantitatively in those terms in future work, combining quantitative thermalization rate and hot-electron group velocity as a function of energy to calculate hot $D_{e}\left(T_{e}, t\right)$ over the time and electron temperature interval of nonlinear quenching. For the time being, we have represented the hot electron diffusion coefficient as proportional to the square root of the calculated group velocity over the range of phonon cooling of hot electrons, and simply use hot electron group velocity $v_{g}$ as the second-level branching parameter on the right side in Fig. 20. [6] The operative rule is illustrated by the schematic cylinder tracks depicted in Fig. 17. Most of the halide scintillators that would be on the right side of the top-level branch also have self-trapped holes. Therefore hot electron diffusion implies wide separation of charges and further implies that de-trapping and diffusion over a relatively long time will be involved to recover radiative recombination of the initially separated hot electrons and self-trapped holes. If hotelectron $v_{g}$ is large, as in $\mathrm{NaI}$ and $\mathrm{CsI}$, the electrons returning toward the track core of self-trapped holes for radiative recombination have a long path through a minefield of defect traps ( $F$ centers, impurities, extended defects) that can prevent useful scintillation light. We could expect that the linear quenched fraction $k_{l}$ may be substantial in such cases. The consequence for a halide hump in electron energy response and lower light in the simple halides like NaI was discussed in connection with Fig. 17. This is represented by the schematic hump in the generic electron energy response curve depicted at the lower right of Fig. 20. If the value of $v_{g}$ and hot electron diffusion range is small, as in crystals with complex unit cell like $\mathrm{SrI}_{2}$ and/or having flat $\mathrm{La} 4 \mathrm{f}$ lower conduction bands like $\mathrm{LaBr}_{3}$, the hot electrons do not go out as far radially and so have a shorter path through the traps toward recombination. The generic predicted response is therefore flatter and higher light yield, both attributable to smaller $k_{l}$ due to smaller diffusion path. Notice that materials in this latter classification, including, e.g. $\mathrm{SrI}_{2}: \mathrm{Eu}, \mathrm{BaBrI}: \mathrm{Eu}$, and $\mathrm{LaBr}_{3}: \mathrm{Ce}$, get positive recommendations for good proportionality and light yield at both levels of branch points: At the first branching, free carrier dominance in this group leads to pure $3^{\text {rd }}$ order quenching, which has inherently a flatter shape over most of the photon density response. This implies flatter electron energy response and higher light yield. Then at the second-level branch point, small hotelectron diffusion range (small $v_{g}$ ) implies smaller linear quenched fraction $k_{l}$ and this means flatter electron energy response and higher light yield yet again. The left second-level branch under the right top-level branch is doubly blessed and comprises a sweet spot in material parameter space for scintillator proportionality and light yield.

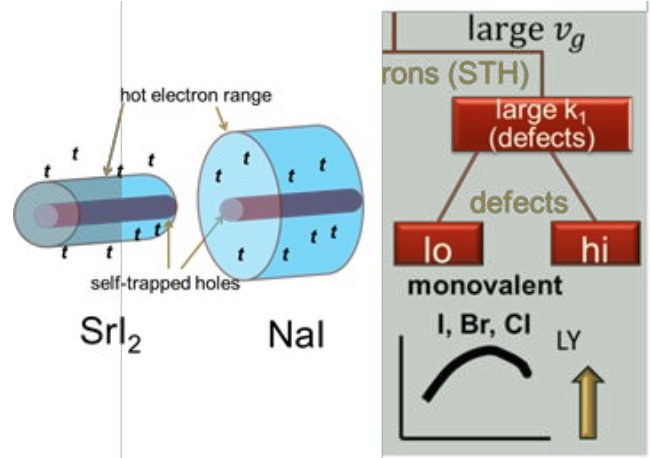

Figure 20. Second level branching on the right side according to hot electron group velocity $v_{g}$, standing in for hot electron diffusion coefficient. Existence of self-trapped holes in most halides and concentration of defects also play roles as discussed. 


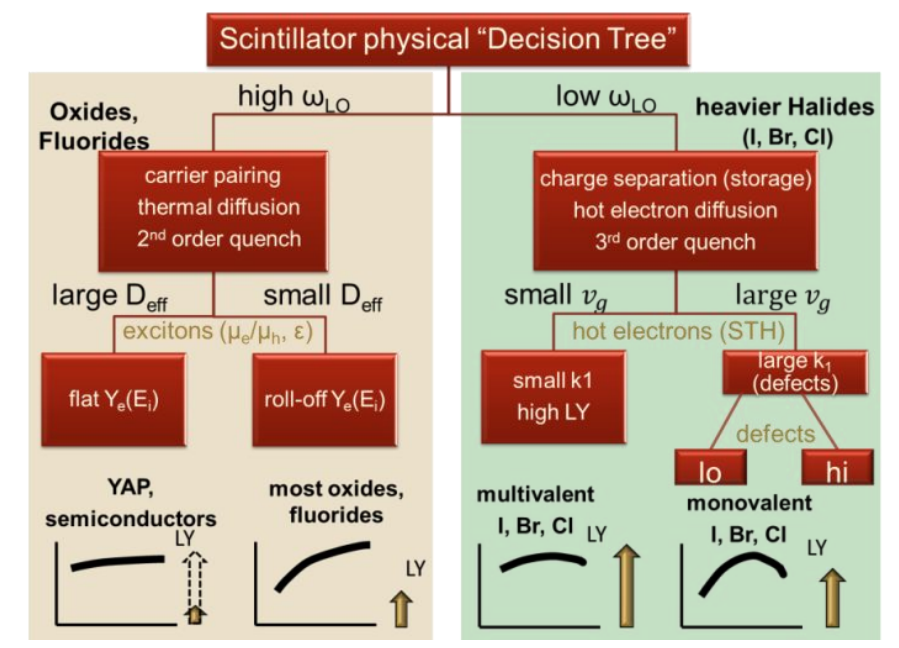

Figure 21. Assembly of all the branching points considered for this decision tree comprising the start of a design rule for proportionality and light yield of inorganic scintillators.

Figure 21 assembles all branches together in one diagram. It was presented previously in Ref. [6], without as much supporting discussion.

Acknowledgment: Supported by NNSA, Office of Nonproliferation Research and Development (NA-22) contracts DENA0001012 and DE-AC02-05CH11231. We thank Steve Payne, Bill Moses, Andrey Vasil'ev and Jai Singh for helpful discussions.

\section{REFERENCES}

[1] S. A. Payne, N. J. Cherepy, G. Hull, J. D. Valentine, W. W. Moses, and W.-S. Choong, "Nonproportionality of Scintillator Detectors: Theory and Experiment," IEEE Transactions on Nuclear Science 56(4), 2506-2512 (2009) [doi:10.1109/TNS.2009.2023657].

[2] S.A. Payne, W.W. Moses, S. Sheets, L. Ahle, N.J. Cherepy, B. Sturm, S. Dazeley, G. Bizarri, Choong WoonSeng, "Nonproportionality of Scintillator Detectors: Theory and Experiment. II," Nuclear Science, IEEE Transactions on 58(6), 3392-3402 (2011).

[3] I. V Khodyuk, P. A. Rodnyi, and P. Dorenbos, "Nonproportional scintillation response of NaI:Tl to low energy x-ray photons and electrons," J. Appl. Phys. 107(11), 113513, AIP (2010) [doi:10.1063/1.3431009].

[4] Z. Wang, Y. Xie, B. D. Cannon, L. W. Campbell, F. Gao, and S. Kerisit, "Computer simulation of electron thermalization in CsI and CsI(Tl)," J. Appl. Phys. 110(6), 64903, AIP (2011) [doi:10.1063/1.3632969].

[5] Z. Wang, Y. Xie, L. W. Campbell, F. Gao, and S. Kerisit, "Monte Carlo simulations of electron thermalization in alkali iodide and alkaline-earth fluoride scintillators," Journal of Applied Physics 112(1), 014906, American Institute of Physics (2012) [doi:10.1063/1.4736088].

[6] Q. Li, J. Q. Grim, K. B. Ucer, A. Burger, G. A. Bizarri, W. W. Moses, and R. T. Williams, "Host structure dependence of light yield and proportionality in scintillators in terms of hot and thermalized carrier transport," Phys. Status Solidi RRL 6(8), 346-348, WILEY-VCH Verlag (2012) [doi:10.1002/pssr.201206258].

[7] R. Kirkin, V. V Mikhailin, and A. N. Vasil'ev, "Recombination of Correlated Electron-Hole Pairs With Account of Hot Capture With Emission of Optical Phonons," IEEE Trans. Nucl. Sci. 59(5), 2057-2064 (2012) [doi:10.1109/TNS.2012.2194306].

[8] A. Kozorezov, J. K. Wigmore, and A. Owens, "Picosecond dynamics of hot carriers and phonons and scintillator non-proportionality," J. Appl. Phys. 112(5), 53709, AIP (2012) [doi:10.1063/1.4749253].

[9] J. Q. Grim, K. B. Ucer, A. Burger, P. Bhattacharya, E. Tupitsyn, E. Rowe, V. M. Buliga, L. Trefilova, A. Gektin, et al., "Nonlinear quenching of densely excited states in wide-gap solids," Physical Review B 87(12), 125117, American Physical Society (2013) [doi:10.1103/PhysRevB.87.125117].

[10] M. Sheik-Bahae, A. A. Said, T.-H. Wei, D. J. Hagan, and E. W. Van Stryland, "Sensitive measurement of optical nonlinearities using a single beam," Quantum Electronics, IEEE Journal of 26(4), 760-769 (1990) [doi:10.1109/3.53394]. 
[11] I. V Khodyuk and P. Dorenbos, "Trends and Patterns of Scintillator Nonproportionality," IEEE Trans. Nucl. Sci. 59(6), 3320-3331 (2012) [doi:10.1109/TNS.2012.2221094].

[12] M. Moszynski, "Energy Resolution and Non-proportionality of Scintillation Detectors," MRS Proceedings 1038, 1038-O07-02, Cambridge University Press (2011) [doi:10.1557/PROC-1038-O07-02].

[13] A. Vasil'ev, "Elementary processes in scintillation and their interconnection in scintillation process," in Proceedings of the 8th International Conference on Inorganic Scintillators and their Applications National Academy of Sciences of Ukraine, Kharkov, 1-6 (SCINT 2005), A. Gektin and B. Grinyov, Eds., pp. 1-6, National Academy of Sciences of Ukraine, Kharkov (2006).

[14] A. Vasil'ev and A. Gektin, "Multiple scale approach to estimation of scintillator characteristics," in SCINT2013, Shanghai, China (2013).

[15] Q. Li, J. Q. Grim, N. A. W. Holzwarth, and R. T. Williams, "Hot electron velocity, relaxation time, and range in ultraviolet- and electron-excited scintillators and semiconductors," in SCINT2013 (2013).

[16] R. T. W. K.B. Ucer, J.Q. Grim, A. Burger, A. Gektin, L. Trefilova, "Early Populations and Trapping Rates in Iodide Scintillators Studied by Picosecond Optical Absorption," in SCINT2013 (2013).

[17] R. T. Williams, K. B. Ucer, J. Q. Grim, K. C. Lipke, L. M. Trefilova, and W. W. Moses, "Picosecond Studies of Transient Absorption Induced by BandGap Excitation of CsI and CsI:Tl at Room Temperature," IEEE Trans. Nucl. Sci. 57(3), 1187-1192 (2010) [doi:10.1109/TNS.2009.2033184].

[18] S. Kerisit, Z. Wang, R. T. Williams, J. Q. Grim, and and F. Gao, "Kinetic Monte Carlo Simulations of Scintillation Processes in NaI(Tl)," under review, IEEE Trans. Nucl. Sci. (2013).

[19] Z. Wang, R. T. Williams, J. Q. Grim, F. Gao, and S. Kerisit, "Kinetic Monte Carlo simulations of excitation density dependent scintillation in CsI and CsI(Tl)," physica status solidi (b), n/a-n/a (2013) [doi:10.1002/pssb.201248587].

[20] R. Murray and A. Meyer, "Scintillation Response of Activated Inorganic Crystals to Various Charged Particles," Physical Review 122(3), 815-826, American Physical Society (1961) [doi:10.1103/PhysRev.122.815].

[21] J. B. Birks, The Theory and Practice of Scintillation Counting, p. 662, Pergamon Press (1964).

[22] R. T. Williams, J. Q. Grim, Q. Li, K. B. Ucer, G. A. Bizarri, and A. Burger, "Scintillation Detectors of Radiation: Excitations at High Densities and Strong Gradients," in Excitonic and Photonic Processes in Materials, J. Singh and R. T. Williams, Eds., Springer-Verlag, Heidelberg (2013).

[23] K. S. Song and R. T. Williams, Self-trapped excitons, Second, Springer-Verlag, Heidelberg (1996).

[24] D. Aberg, P. Erhart, and B. Sadigh, "First principles study of self-trapped excitons in NaI and SrI2," To be published.

[25] J. Q. Grim, Q. Li, K. B. Ucer, R. T. Williams, G. A. Bizarri, and W. W. Moses, "Electron energy response of NaI:Tl and SrI2:Eu calculated from carrier mobilities and measured first- and third-order quenching," Mater. Res. Soc. Communications Research Letters 2(04), 139-143 (2012) [doi:10.1557/mrc.2012.27].

[26] R. T. Williams, J. Q. Grim, Q. Li, K. B. Ucer, and W. W. Moses, "Excitation density, diffusion-drift, and proportionality in scintillators," physica status solidi (b) 248(2), 426-438 (2011) [doi:10.1002/pssb.201000610].

[27] Q. Li, J. Q. Grim, R. T. Williams, G. A. Bizarri, and W. W. Moses, "A transport-based model of material trends in nonproportionality of scintillators," J. Appl. Phys. 109(17), 123716, AIP (2011) [doi:10.1063/1.3600070].

[28] J. Q. Grim, Q. Li, K. B. Ucer, A. Burger, G. A. Bizarri, W. W. Moses, and R. T. Williams, "The roles of thermalized and hot carrier diffusion in determining light yield and proportionality of scintillators," physica status solidi (a) 209(12), 2421-2426, WILEY-VCH Verlag (2012) [doi:10.1002/pssa.201200436].

[29] R. T. Williams, Q. Li, J. Q. Grim, and K. B. Ucer, "Dependence of nonproportionality in scintillators on diffusion of excitons and charge carriers," in SPIE Optical Engineering + Applications, L. A. Franks, R. B. James, and A. Burger, Eds., p. 81420Y-81420Y-17, International Society for Optics and Photonics (2011) [doi:10.1117/12.895038].

[30] F. Gao, Y. Xie, S. Kerisit, L. W. Campbell, and W. J. Weber, "Yield, variance and spatial distribution of electron-hole pairs in CsI," Nuclear Instruments and Methods in Physics Research Section A: Accelerators, Spectrometers, Detectors and Associated Equipment 652(1), 564-567 (2011) [doi:10.1016/j.nima.2010.08.063].

[31] G. A. Bizarri, W. W. Moses, J. Singh, A. N. Vasil'ev, and R. T. Williams, "An analytical model of nonproportional scintillator light yield in terms of recombination rates," J. Appl. Phys. 105(15), 44507, AIP (2009) [doi:10.1063/1.3081651].

[32] J. Q. Grim, K. B. Ucer, A. Burger, G. A. Bizarri, and R. T. Williams, "SrI2 track radius," To be published.

[33] W. Martienssen, "Uber die excitonenbanden der alkalihalogenidkristalle," Journal of Physics and Chemistry of Solids 2(4), 257-267 (1957) [doi:10.1016/0022-3697(57)90070-7]. 
[34] P. Dorenbos, "Fundamental Limitations in the Performance of Ce3+-, Pr3+-, and Eu2+- Activated Scintillators," IEEE Trans. Nucl. Sci. 57(3), 1162-1167 (2010) [doi:10.1109/TNS.2009.2031140].

[35] W. Setyawan, R. M. Gaume, S. Lam, R. S. Feigelson, and S. Curtarolo, "High-throughput combinatorial database of electronic band structures for inorganic scintillator materials.," ACS combinatorial science 13(4), 382-390 (2011) [doi:10.1021/co200012w].

[36] S. Marchetti, M. Martinelli, and R. Simili, "The Auger recombination coefficient in InAs and GaSb derived from the infrared dynamical plasma reflectivity," Journal of Physics: Condensed Matter 14(13), 3653 (2002).

[37] U. Strauss, W. W. Rühle, and K. Köhler, "Auger recombination in intrinsic GaAs," Appl. Phys. Lett. 62(1), 5557, AIP (1993) [doi:10.1063/1.108817].

[38] Y. C. Shen, G. O. Mueller, S. Watanabe, N. F. Gardner, A. Munkholm, and M. R. Krames, "Auger recombination in InGaN measured by photoluminescence," Appl. Phys. Lett. 91(14), 141101, AIP (2007) [doi:10.1063/1.2785135]. 


\title{
Monte Carlo simulation of gamma-ray response of $\mathrm{BaF}_{\mathbf{2}}$ and $\mathrm{CaF}_{\mathbf{2}}$
}

\author{
F. Gao, ${ }^{\text {a) }}$ Y. L. Xie, ${ }^{\text {a) }}$ Z. G. Wang, S. Kerisit, D. X. Wu, L. W. Campbell, R. M. Van Ginhoven, \\ and M. Prange \\ Pacific Northwest National Laboratory, P.O. Box 999, Richland, Washington 99352, USA
}

(Received 12 September 2013; accepted 17 October 2013; published online 7 November 2013)

\begin{abstract}
We have employed a Monte Carlo (MC) method to study intrinsic properties of two alkaline-earth halides, namely, $\mathrm{BaF}_{2}$ and $\mathrm{CaF}_{2}$, relevant to their use as radiation detector materials. The $\mathrm{MC}$ method follows the fate of individual electron-hole (e-h) pairs and thus allows for a detailed description of the microscopic structure of ionization tracks created by incident $\gamma$-ray radiation. The properties of interest include the mean energy required to create an e-h pair, $W$, Fano factor, $F$, the maximum theoretical light yield, and the spatial distribution of e-h pairs resulting from $\gamma$-ray excitation. Although $W$ and $F$ vary with incident photon energy at low energies, they tend to constant values at energies higher than $1 \mathrm{keV} . W$ is determined to be 18.9 and $19.8 \mathrm{eV}$ for $\mathrm{BaF}_{2}$ and $\mathrm{CaF}_{2}$, respectively, in agreement with published data. The e-h pair spatial distributions exhibit a linear distribution along the fast electron tracks with high e-h pair densities at the end of the tracks. Most e-h pairs are created by interband transition and plasmon excitation in both scintillators, but the e-h pairs along fast electron tracks in $\mathrm{BaF}_{2}$ are slightly clustered, forming nanoscale domains and resulting in the higher e-h pair densities than in $\mathrm{CaF}_{2}$. Combining the maximum theoretical light yields calculated for $\mathrm{BaF}_{2}$ and $\mathrm{CaF}_{2}$ with those obtained for $\mathrm{CsI}$ and $\mathrm{NaI}$ shows that the theoretical light yield decreases linearly with increasing band gap energy. (c) 2013 AIP Publishing LLC. [http://dx.doi.org/10.1063/1.4828718]
\end{abstract}

\section{INTRODUCTION}

Investigation of the interaction of $\gamma$-rays with inorganic materials is of great significance for many fields. Because of their practical applications to medical imaging, radiation detector physics, and security inspections, there is considerable interest in the development of new radiation detector materials that can be used at room temperature to identify $\gamma$-rays from radioactive isotopes. Scintillators offer numerous advantages as $\gamma$-ray radiation detection materials including high light yields, fast response, large active areas, and roomtemperature operation. ${ }^{1}$ In general, the interaction of $\gamma$-rays with scintillator materials results in the creation of fast electrons that can interact further with the material to create electron-hole (e-h) pairs. These secondary electrons (SEs) lose their energy through a complex cascade of energy transfer interactions that culminates in the creation of a large number of conduction electrons and valence holes that lack the energy for further electronic excitations. An important characteristic of detector materials is their efficiency for secondary electron production following their irradiation by photons and high-energy particles, from which some intrinsic properties such as the mean energy required to create an electron-hole pair, $W$, and the Fano factor, $F$, can be determined. It should be noted that Fano factor defined here is used to describe the variance in the number of electron-hole pairs, which might be different from the photon statistics defined in most scintillation experiments. These intrinsic properties can provide important insights into the factors that

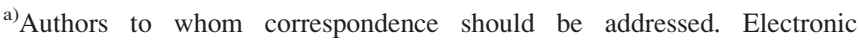
addresses: fei.gao@pnnl.gov and yulong.xie@pnnl.gov
}

control the fundamental performance limits of these materials, such as the achievable energy resolution, and explain why some materials perform better than others.

It is well known that many inorganic scintillator materials exhibit a nonlinear scintillation response (commonly referred to as "nonproportionality"), which is one of the main sources degrading the intrinsic energy resolution achievable with these materials. ${ }^{2}$ Previous studies of scintillator materials by Dorenbos et al. ${ }^{3}$ and Payne et al. ${ }^{4}$ demonstrated that nonproportionality is ultimately correlated to the energy dependence of the density of electron-hole pairs along ionization tracks. Thus, a fundamental understanding of ionization track structures created by the interaction of high-energy particles with inorganic scintillators is crucial to successfully model the nonproportional response of scintillator materials. Experimental studies of the spatial distribution and track structure of electron-hole pairs are complicated and yield only qualitative estimations. Our current understanding of the spatial distribution of e-h pairs comes mainly from theoretical studies and computer simulations. Previously, several Monte Carlo (MC) models have been developed to approximate the spatial distribution of e-h pairs in CsI. ${ }^{5-8}$ In this work, a Monte Carlo code, NWEGRIM (Northwest Electron and Gamma Ray Interaction in Matter), is used to simulate electron cascades in $\mathrm{BaF}_{2}$ and $\mathrm{CaF}_{2}$ resulting from interactions with $\gamma$-rays with initial energies ranging from $50 \mathrm{eV}$ to $1 \mathrm{MeV}$. The $\mathrm{MC}$ code includes the ability to follow the energy cascade down to energies on the order of the band gap, where the cross sections of electrons at low energy are fitted to $a b$ initio calculations. $\mathrm{BaF}_{2}$ and $\mathrm{CaF}_{2}$ are two of the most important fluoride scintillator materials for $\gamma$-ray detectors and have been the subject of many 
experimental studies. ${ }^{9,10}$ In addition to the calculations of $W$, variance, and fundamental performance limits, the current MC code was also developed to study the spatial distribution of electron-hole pairs induced by photons and electrons in detector materials and has been employed to obtain the details of the spatial distribution of electron-hole pairs in $\mathrm{BaF}_{2}$ and $\mathrm{CaF}_{2}$. The microscopic distribution of electron-hole pairs will allow for further simulations of electron thermalization in scintillator materials ${ }^{11}$ and for large-scale simulations of electron-hole pair transport that explore the possible origins of nonproportionality. ${ }^{12}$

\section{METHODOLOGY}

The simulation is started by creating an incident photon with a specific energy, $E_{p}$, and its interaction with an atom may involve inner-shell photoionization, Compton scattering, or electron-positron pair production, resulting in fast electron emission. The relative probability of each event can be determined from the corresponding cross sections. ${ }^{13-15}$ The energy loss of a fast electron, as well as the creation of SEs, occurs through a number of possible quantum mechanical mechanisms. Several energy loss mechanisms are considered, which include valence to conduction interband transitions, plasmon excitations, core shell ionizations, electron-phonon interactions, and Bremsstrahlung emission. Fig. 1 shows gamma-ray interaction with materials to create fast electrons and all the energy loss mechanisms of fast electrons considered in the simulation. Given the energy $E_{e}$ of an electron, the probability of a process occurring is equal to its inverse mean free path (IMFP) divided by the total IMFP at that energy. To determine the location of ionization events and the atomic shell that is ionized, the cross sections or IMFPs for ionization of individual inner shells in $\mathrm{BaF}_{2}$ and $\mathrm{CaF}_{2}$ are calculated using an optical-data model of the generalized oscillator strength (GOS) developed by Mayol and Salvat. ${ }^{16}$ The core hole created will then decay through a vacancy-cascade process involving the emission of Auger/Coster-Kronig or fluorescence photons and possible shake-off electron emission. The possible relative relaxation pathways through these non-radiative and radiative transitions are extracted from the LLNL Evaluated Atomic Data Library ${ }^{13}$ while the probabilities for electron shake-off after the creation of an inner shell vacancy are approximated by those for the rare gases. ${ }^{17}$ A model to evaluate the electron mean free paths of interband transition and plasmon excitation over a wide energy range (from several $\mathrm{eV}$ to a few

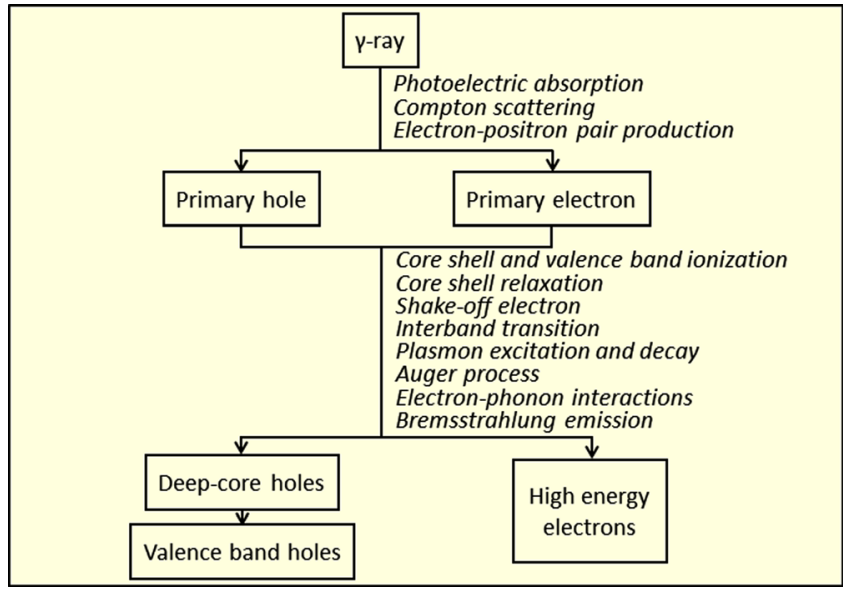

FIG. 1. Schematic sketch showing gamma-ray interaction with materials to create fast electrons and all the energy loss mechanisms of fast electrons considered.

hundred $\mathrm{MeV}$ ) in a relativistic kinematics framework has been developed, where the cross sections at low energy are fitted to an $a b$ initio data model ${ }^{18}$ for band structure to be considered. Ab initio calculations of the electron mean free paths in $\mathrm{BaF}_{2}$ and $\mathrm{CaF}_{2}$ will be reported in detail elsewhere; thus, only the central principles are described here.

\section{A. Ab initio calculations}

We have used the ABNIT code ${ }^{19}$ to perform band structure calculations. The orbital wavefunctions $\psi_{\mathrm{n} \kappa}$ (r) for band $\mathrm{n}$ and wave vector $\kappa$ are taken as the Kohn-Sham eigenvectors. The orbital energies are the density functional theory (DFT) eigenvalues corrected with the $G W$ approximation of Hedin, where $G$ stands for the one-electron Green's function and $W$ is the screened potential of an electron. With these calculations, the polarizability $\alpha$ and dielectric function $\varepsilon$ can be determined, which are the basis for calculating the differential energy loss cross sections, inelastic mean free paths of electrons and holes, and their secondary spectra. These quantities are required input for the NWEGRIM code. The dielectric response of a crystal can be calculated for a given transfer of energy $\omega$ and crystal momentum within the reciprocal unit cell $\boldsymbol{q}$ for a matrix of reciprocal lattice vectors $\boldsymbol{K}, \boldsymbol{K}^{\prime}$ via $^{20,21}$

$$
\varepsilon_{K K^{\prime}}(\bar{q}, \omega)=1-4 \pi \alpha_{K K^{\prime}}(\bar{q}, \omega),
$$

where

$$
\alpha_{K K^{\prime}}(\bar{q}, \omega)=\frac{1}{|\bar{q}+\bar{K}|\left|\bar{q}+\bar{K}^{\prime}\right|} \sum_{c}^{\text {unocc occ }} \sum_{v} \int \frac{d^{3} k}{(2 \pi)^{3}}\left[\frac{\rho_{c v}(\bar{k}, \bar{q}+\bar{K}) \rho_{c v}^{*}\left(\bar{k}, \bar{q}+\bar{K}^{\prime}\right)}{\omega-\varepsilon_{c}(\bar{k})+\varepsilon_{v}(\bar{k}-\bar{q})+i \eta}-\frac{\rho_{v c}(\bar{k}, \bar{q}+\bar{K}) \rho_{v c}^{*}\left(\bar{k}, \bar{q}+\bar{K}^{\prime}\right)}{\omega+\varepsilon_{c}(\bar{k})-\varepsilon_{v}(\bar{k}-\bar{q})+i \eta}\right],
$$

and

$$
\rho_{n m}(\bar{k}, \bar{q})=\int d^{3} r \psi_{n \bar{k}}(r) \psi_{m(\bar{k}-\bar{q})}^{*}(r) e^{-i \bar{q} \cdot \bar{k}}
$$

where $\eta$ is a positive infinitesimal. The energy loss function (ELF), which describes interband transitions and plasmon excitations, can be evaluated as matrix inverse of $\varepsilon_{\boldsymbol{K} \boldsymbol{K}^{\prime}}$ for each $q$ and $\omega$. Our $a b$ initio calculations indicate that the 
plasmon excitation occurs only at low momentum transfer and over a narrow range of energies. We have generalized the work of Chung and Everhart ${ }^{22}$ on plasmon decay to all crystalline materials and for arbitrary electronic excitations, as will be described in a separate publication. The e-h pairs resulting from electronic excitations at zero momentum transfer and the free-electron gas plasmon energy were used to estimate the decay spectra of plasmons for $\mathrm{BaF}_{2}$ and $\mathrm{CaF}_{2}$. This replaces the unphysical spikes in electron distribution caused by an empirical model of plasmon decay ${ }^{11}$ with a more realistic distribution of plasmon decay electrons for the first time.

\section{B. Cross sections}

\section{Elastic interactions and electron-phonon interactions}

The cross-sections for elastic collisions are obtained from partial wave calculations, as detailed in Ref. 23, which are accurate for kinetic energies less than $1 \mathrm{MeV}$ (for further details on elastic interaction calculations, see Ref. 23). The interaction of electrons with optical phonons is an inelastic process. In contrast, the electron interaction with acoustic phonons can be treated as quasi-elastic, and the scattering electrons have a nearly isotropic distribution. Thus, we only considered electron interactions (absorption and emission) with optical phonons in this work. The cross sections for the electron-optical phonon interactions are calculated based on the theory of Llacer and Garwin, ${ }^{24}$ as detailed in Ref. 11. Fig. 2 shows the inverse mean free paths obtained for $\mathrm{BaF}_{2}$ and $\mathrm{CaF}_{2}$. The inverse mean free path for phonon emission is larger than that for absorption, and thus it is expected that emission will be more probable. In addition, it is of interest to note that the inverse mean free paths for phonon emission and absorption decrease rapidly with increasing electron energy. Electron-optical phonon interactions are included in the NWEGRIM calculations as they can compete with interband transition at very low electron energies, in the vicinity of the cut-off energy. Below the cut-off energy, where e-h pairs are no longer produced, electron-phonon interactions constitutes the main channel for energy loss of electrons until they reach thermal energy. This thermalization stage has been the subject of separate work by our group ${ }^{11,25}$ and

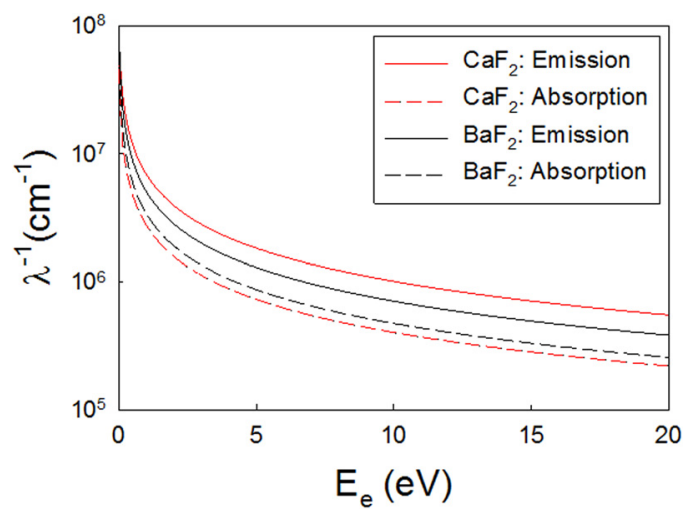

FIG. 2. Inverse mean free paths for optical phonon emission and absorption in $\mathrm{CaF}_{2}$ and $\mathrm{BaF}_{2}$. others, ${ }^{26,27}$ but is beyond the scope of this work as we concentrate here on the electron-hole pair generation stage.

\section{Interband transitions and plasmon excitations}

Based on the GOS method, we have developed a model to evaluate the electron mean free paths of interband transition and plasmon excitation over a wide energy range (from several $\mathrm{eV}$ to a few hundred $\mathrm{MeV}$ ) in a relativistic kinematic framework. According to the GOS model, inelastic collisions of a fast electron with kinetic energy, $E$, with a target atom (atomic number Z) can be described using the energy loss $T$ and the recoil energy $Q$. The recoil energy in relativistic framework is defined by ${ }^{28}$

$$
Q=\left[(c q)^{2}+m^{2} c^{4}\right]^{1 / 2}-m c^{2},
$$

where $m$ is the electron mass, $c$ is the velocity of light, and $\boldsymbol{q}$ is the momentum transfer. To simplify the notation, the differential inelastic cross-section per atom can be written in the form

$$
\frac{d^{2} \sigma}{d T d Q}=\frac{2 \pi e^{4}}{m v^{2}} G(Q, T) \frac{d f(Q, T)}{d T},
$$

where $v$ is the velocity of the incident electron and $d f(Q, T) / d T$ is the GOS per unit energy loss, which is usually referred to as the Bethe surface in the $(Q, T)$ plane. ${ }^{16,29}$ For plasmon excitations, the plasmon-pole approximation, ${ }^{30,31}$ in which the excitation spectrum of the electron gas is replaced by a single mode along the plasma dispersion line, has been proposed to calculate IMFP and stopping power; the results are in good agreement with those obtained from Lindhard's dielectric function ${ }^{32}$ for electron energy larger than plasmon energy. Also, a two-mode model for the plasmon dispersion line has been suggested by Martinez et al. ${ }^{33}$ to reproduce the linear behavior of the Lindhard differential cross section (DCS) for low energy loss and has been shown to be an improvement over the single pole approximation. Similarly, the plasmon dispersion line in a material is proposed to be

$$
T_{Q}^{2}=E_{p}^{2}+E_{g}^{2}+2 \mu^{2} Q+Q^{2}
$$

where $\mu^{2}=6 E_{F} / 5$. The cut-off energy loss $T_{P}$ (or $Q_{P}$ ) is maximum energy (or recoil energy) transferred to a plasmon without degenerating into electron-hole pairs. Within a limited region of small $Q\left(\leq Q_{p}\right)$, plasmon excitations and interband transitions coexist while for large $Q$ the plasmon excitation disappears, and electron-hole excitation by interband transitions continues into the Bethe ridge $(T=Q)$. The partition of these two branches can be described by strength $g(Q)$

$$
g(Q)=\min \left\{1, A\left[\frac{Q}{E_{p}}\right]^{B}\right\},
$$

where $A$ and $B$ are materials-dependent coefficients. The explicit forms of the parameters $A$ and $B$ can be fitted to experimental data or to the results of first-principles 
calculations at low energies. The excitation spectrum of the outer-shell oscillator can be described by

$$
F(Q, T)=[1-g(Q)] \delta\left(T-T_{Q}\right)+g(Q) \delta(T-Q),
$$

where the first term describes plasmon excitations and the second term along the line $T=Q$ corresponds to interband electron-hole excitations. It is convenient to express the DCS per electron (Eq. (5)) associated with $F(Q, T)$ in Eq. (8) as the sum of contributions from electron-hole and plasmon excitations

$$
\frac{d^{2} \sigma_{v}}{d T d Q}=\frac{d^{2} \sigma_{p l}}{d T d Q}+\frac{d^{2} \sigma_{e h}}{d T d Q}
$$

Relativistic effects on the DSC for interband transitions can be described by the Born relativistic DCSs for binary collisions with free electrons at rest. ${ }^{34}$ Exchange effects can be included by using the Møller formula, ${ }^{35}$ and the relativistic DCS for electron-hole excitation can then be written in the form

$$
\frac{d^{2} \sigma_{e h}}{d T d Q}=\frac{2 \pi e^{4}}{m v^{2}} G^{-}(Q, T) g(Q) \delta(T-Q) .
$$

The function $G^{-}(Q, T)$ is given by

$$
\begin{aligned}
G^{-}(Q, T)= & \frac{1}{T Q}\left[1+\left(\frac{Q}{E-T}\right)^{2}-\frac{Q}{E-T}\right. \\
& \left.+\left(\frac{\gamma-1}{\gamma}\right)\left(\frac{Q^{2}}{E^{2}}-\frac{Q}{E-T}\right)\right],
\end{aligned}
$$

where $\gamma=1+\mathrm{E} / \mathrm{mc}^{2}$.

The relativistic DCS per electron from plasmon excitations can be described using the first Born approximation ${ }^{25}$ and can be written in the form

$$
\frac{d^{2} \sigma_{p l}}{d T d Q}=\frac{2 \pi e^{4}}{m v^{2}} G^{+}(Q, T)[1-g(Q)] \delta\left(T-T_{Q}\right)
$$

and

$$
G^{+}(Q, T)=\frac{1}{T Q\left(1+Q / 2 m c^{2}\right)}+\frac{\beta_{t}^{2} T / 2 m c^{2}}{\left[Q\left(1+Q / 2 m c^{2}\right)-T^{2} / 2 m c^{2}\right]^{2}},
$$

where $\beta_{t}$ is the component of $\boldsymbol{\beta}=\boldsymbol{v} / c$ perpendicular to the momentum transfer. The energy loss DCS can be obtained by integrating Eq. (9) over the interval of recoil energies allowed by energy and momentum conservation. The corresponding energy loss DCSs are given by

$$
\begin{gathered}
\frac{d \sigma_{e h}}{d T}=\frac{2 \pi e^{4}}{m v^{2}} G^{-}(T) g(T), \\
\frac{d \sigma_{p l}}{d T}=\frac{2 \pi e^{4}}{m v^{2}} \frac{T}{\left(T^{2}-E_{p}^{2}-E_{g}^{2}+\mu^{4}\right)^{1 / 2}} G^{+}\left(Q_{0}, T\right)\left[1-g\left(Q_{0}\right)\right],
\end{gathered}
$$

where $Q_{0}$ in Eq. (15) is determined by the plasmon dispersion line of Eq. (6). The individual cross section for electron-hole and plasmon excitations is obtained by integrating Eqs. (14) and (15) from $T_{\min }$ to $T_{\max }$. The minimum energy, $T_{\min }$, for plasmon excitation is fixed by the intersection of the dispersion line of Eq. (6), and the recoil energies allowed by energy and momentum conservation, and the maximum energy, $T_{\max }$, is taken as $T_{p}$. The $T_{\min }$ for electron-hole excitation can be set to zero while the maximum energy allowed energy transfer is limited to $T_{\max }=E / 2$ because of two indistinguishable free electrons involved. This means that the effective threshold, $E_{t h}$, of an incident electron for interband transitions occurring is at least twice the band gap energy. The mean energy loss involved in plasmon excitation by a fast electron with energy $E_{e}$ can be calculated by

$$
E_{\text {loss }}=\int T \frac{d \sigma_{p l}}{d T} d T / \int \frac{d \sigma_{p l}}{d T} d T
$$

Energy loss due to plasmon creation by an incident electron with $E_{e}$ is then determined by

$$
E_{e}^{\prime}=E_{e}-E_{\text {loss }} .
$$

The subsequent plasmon decay process and the energies of new charge carriers will be determined by the plasmon decay spectra obtained by ab initio calculations. The new charge carriers may be capable of generating further electron-hole pairs if their energies are larger than the threshold for interband transition. A random sampling algorithm is employed to obtain the energy loss of an incident electron due to an interband transition, where the probability distribution function (PDF) of the reduced energy loss $\kappa=E_{\text {loss }} / E_{e}$ is proportional to the energy loss differential cross sections of interband transition (see Ref. 36 for further details). It is also possible that the energy loss due to interband transition can be directly chosen by the normalized probability distribution calculated using energy loss differential cross section, as discussed in Ref. 37. However, these methods have been carefully checked, and the simulation results are statistically similar.

\section{Simulation approach}

To calculate the production and spatial distribution of electron-hole pairs, the $\mathrm{MC}$ code described above has been applied to two scintillator materials, $\mathrm{BaF}_{2}$ and $\mathrm{CaF}_{2}$. The energy losses to plasmon excitations, interband transitions, core shell ionizations, and electron-phonon interaction have been simulated in detail. The trajectories of particles have been simulated including all generations of secondary electrons and holes, Auger electrons, shake-off electrons, and the primary electron. The trajectory is stopped when the electron energy is less than the cut-off energy, which is twice the band gap of the material of interest. Calculations were carried out for 44 photon energies ranging from $50 \mathrm{eV}$ to $1 \mathrm{MeV}$, and the number of photon events simulated at each energy was equal to $10^{5}$ to ensure convergence of the electron number distributions and of the calculated values of $F$ 

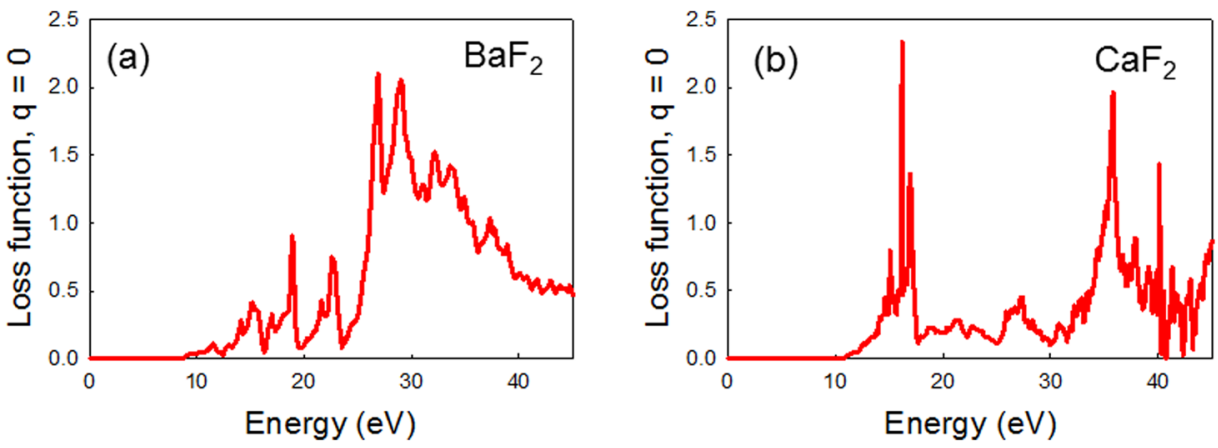

FIG. 3. Energy loss function as a function of electron energy for (a) $\mathrm{BaF}_{2}$ and (b) $\mathrm{CaF}_{2}$.

and $W$. This approach allows the evaluation of the achievable energy resolution in these materials. A number of electron histories were stored for further analysis of both the electron density along the tracks and the final energy distribution.

\section{RESULTS AND DISCUSSION}

\section{A. Dielectric functions and total cross sections}

Band structure calculations for the two materials were performed with the fluorite cubic structure, space group Fm$3 \mathrm{~m}$, and lattice parameters equal to $0.619 \mathrm{~nm}$ for $\mathrm{BaF}_{2}$ and $0.546 \mathrm{~nm}$ for $\mathrm{CaF}_{2}$. Initial valence electron configurations as $\mathrm{Ba}:\left[3 p^{6} 4 s^{2}\right], \mathrm{Ca}:\left[5 p^{6} 6 s^{2}\right]$, and F:[1 $\left.p^{5} 2 s^{2}\right]$ were used. The electronic structure is calculated on a regular grid in the first Brillouin zone, and integration of the electronic response is carried out using the tetrahedron method, ${ }^{38}$ thus avoiding convergence problems when attempting to integrate the rapidly varying energy denominator near resonance. The screening produced by this electronic response, along with the calculated eigenvalues and eigenvectors of the electronic structure, was used to calculate the electronic lifetimes (and hence mean free paths and cross sections) of charge carriers (electrons and holes) in the materials. Figs. 3(a) and 3(b) show the energy loss function of $\mathrm{BaF}_{2}$ and $\mathrm{CaF}_{2}$, respectively. It clearly indicates that the loss function has a complicated plasmon structure between 10 and $50 \mathrm{eV}$. From these calculations, the cross sections of electrons at low energies will be determined, as detailed below. In addition, the energy distribution of the conduction electrons is determined as part of the response function integration, and the results are shown in Fig. 4. The distribution of plasmon decay electrons in both materials exhibits several narrow peaks. Previously, Fraser et al. $^{39}$ and Gao et al. $^{36}$ assumed that each plasmon decays to a single plasmon excitation energy, which leads to a large spike in the kinetic energy distribution of electrons in electron cascades. ${ }^{11}$ It is believed that the new plasmon decay spectra will eliminate these unphysical energy distributions in $\mathrm{BaF}_{2}$ and $\mathrm{CaF}_{2}$. The cross sections for interband transitions, plasmon excitation, and inner-shell ionizations can be numerically integrated by the trapezoidal rule and are presented in Fig. 5 as a function of electron energy measured from the top of the valence band, along with ab initio calculations for comparison. The cross sections at low energies are fitted to $a b$ initio calculations, and the fitting parameters $A$ and $B$ are 2.0 and 3.56 for $\mathrm{BaF}_{2}$ and 2.0 and 3.2 for $\mathrm{CaF}_{2}$, respectively. The analytical cross sections at low energy regions are in good agreement with those determined by $a b$ initio calculations.

\section{B. Intrinsic properties}

Several intrinsic properties, such as $W, F$, and the variance in the number of e-h pairs produced, can be directly determined from a series of simulations at each photon energy. The electron number distributions in $\mathrm{CaF}_{2}$ and $\mathrm{BaF}_{2}$ are generally similar to those in other scintillator materials, such as CsI (Ref. 5) and NaI, which show approximately a Gaussian distribution, but the numbers of electron-hole pairs created are different. For example, the mean electron number is 5262 and 34662 for 100 and $662 \mathrm{keV}$, respectively, in $\mathrm{BaF}_{2}$, as compared to 4711 and 30934 in $\mathrm{CaF}_{2}$. Using the electron number distributions, the parameters $W$ and $F$ can be calculated, and the results are shown in Fig. 6, where the shell edges are indicated. In general, $W$ shows large fluctuations at low incident energies in both scintillators, similar to the behavior observed in semiconductors, ${ }^{33,40}$ but it becomes constant when the energy is larger than $1 \mathrm{keV}$. The energy resolution and the non-proportionality of the light yield ${ }^{41}$
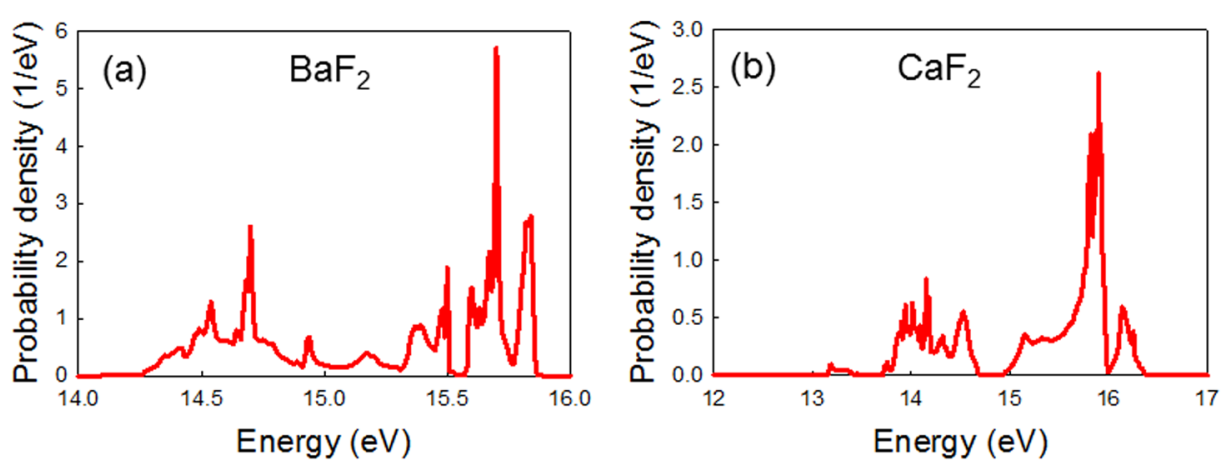

FIG. 4. Energy distribution of the conduction electrons as a function of electron energy for (a) $\mathrm{BaF}_{2}$ and (b) $\mathrm{CaF}_{2}$, as determined as part of the response function integration (Fig. 2). 

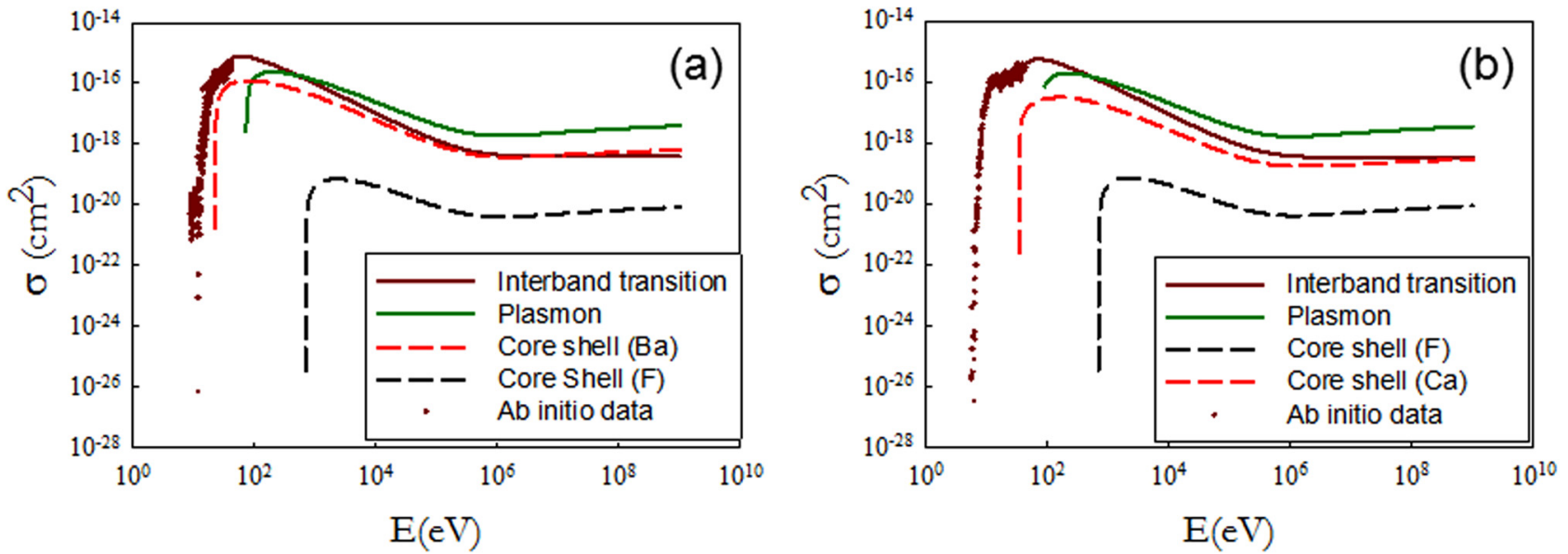

FIG. 5. Cross-sections of core shell ionization, interband transition, and plasmon excitation in (a) $\mathrm{BaF}_{2}$ and (b) $\mathrm{CaF}_{2}$, along with ab initio calculations for comparison.

showed that $\mathrm{BaF}_{2}$ and $\mathrm{CaF}_{2}(\mathrm{Eu})$ exhibited a rise of the relative light yield for energies below $100 \mathrm{keV}$ and $600 \mathrm{keV}$, respectively. The variation of $W$ obtained in the present simulations in both materials suggests that the nonproportionality observed experimentally is not correlated to the number of electron-hole pairs produced (at least for energies higher than $1 \mathrm{keV}) . W$ tends to an asymptotic value of $18.9 \mathrm{eV}$ in $\mathrm{BaF}_{2}$ and $19.8 \mathrm{eV}$ in $\mathrm{CaF}_{2}$, in agreement with other theoretical calculations values of $23.8 \mathrm{eV}$ and $23.4 \mathrm{eV}$ for $\mathrm{BaF}_{2}$ and $\mathrm{CaF}_{2},{ }^{42}$ respectively, but larger than experimental values. ${ }^{43}$ Similarly, $F$ shows fluctuations at low energies and increases with increasing photon energy to a value of 0.2 around $1 \mathrm{keV}$ for $\mathrm{BaF}_{2}$ and 0.22 around $5 \mathrm{keV}$ for $\mathrm{CaF}_{2}$, as shown in Fig. 6. It is clearly seen from Fig. 5 that the cross sections of interband transition and plasmon excitation are similar in both $\mathrm{BaF}_{2}$ and $\mathrm{CaF}_{2}$ and generally larger than those of core shell ionization. Thus, it is expected that the e-h pairs in $\mathrm{BaF}_{2}$ and $\mathrm{CaF}_{2}$ will be mainly created by interband transition and plasmon excitation, thus resulting in small Fano factor.

Using the calculated $W$ values, the maximum theoretical light yield can be estimated for a given scintillator material, which describes the maximum theoretical light yield achievable with this material. Previously, Lempicki and
Wojtowicz ${ }^{44}$ proposed to determine the measured light yield, $\mathrm{L}_{\mathrm{y}}$, using the formula

$$
L_{y}=S Q\left(N_{e-h} / E_{p}\right)
$$

where $S$ stands for the transfer efficiency and $Q$ for the luminescence quantum efficiency, which is often taken to be one. $N_{e-h}$ is the average number of electron-hole pairs produced in electron cascades and $E_{p}$ is the energy of the incident $\gamma$-ray. Assuming both $S$ and $Q$ to be one, the maximum theoretical light yield, which corresponds to every electron-hole pair recombining to emit a photon, can be estimated to be 45122 and $37432 \mathrm{ph} / \mathrm{MeV}$ at $662 \mathrm{keV}$ for $\mathrm{BaF}_{2}$ and $\mathrm{CaF}_{2}$, respectively. The published light yields for pure and Ce-doped $\mathrm{BaF}_{2}$ range from 9950 to $12600 \mathrm{ph} / \mathrm{MeV},{ }^{41,45-48}$ but it should be noted that the light yield also depends on the thickness of samples. ${ }^{9}$ For pure and Eu-doped $\mathrm{CaF}_{2}$, published data show a large variation with absolute light yields ranging from 9580 to $24000 \mathrm{ph} / \mathrm{MeV}$. $^{49}$ The calculated values of theoretical light yields are generally larger than the experimentally absolute light yields in these materials, which is expected as the current calculations do not take into account non-radiative and quenching processes of thermalized
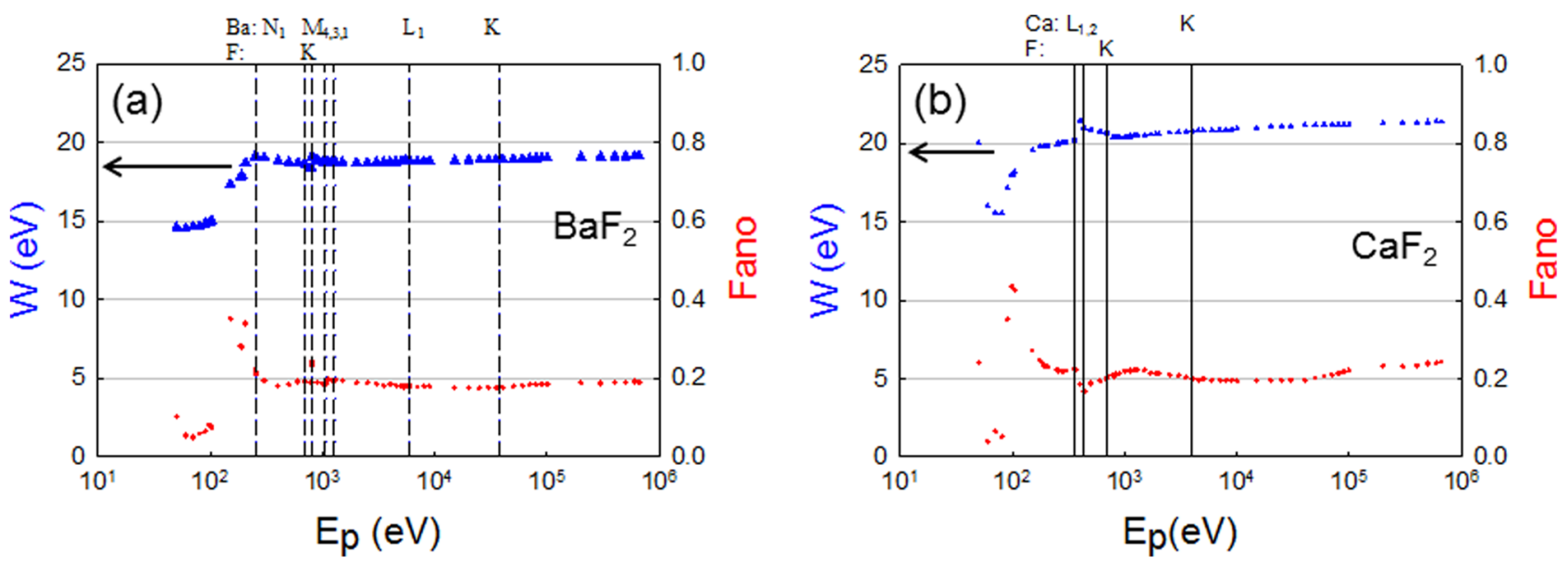

FIG. 6. Mean energy required to create an e-h pair, $W$, and Fano factor, $F$, as a function of photon energy in (a) BaF $F_{2}$ and (b) $\mathrm{CaF}_{2}$, where the ionization energies for different core shells for each element are indicated. 


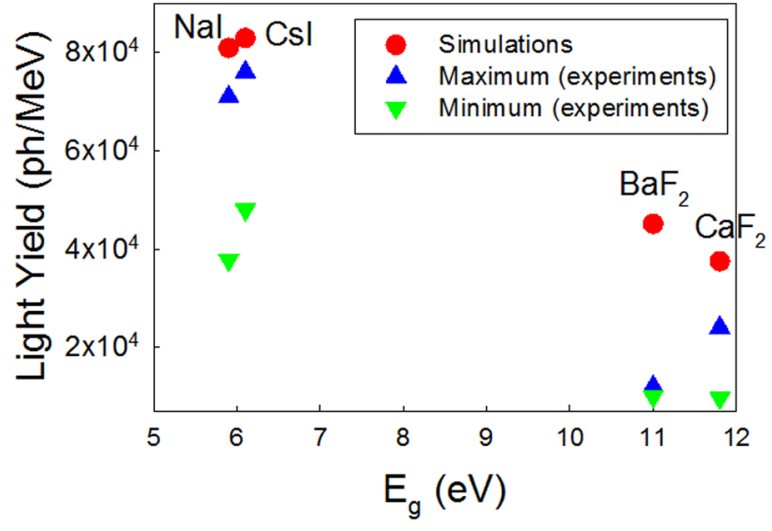

FIG. 7. Maximum theoretical light yield as a function of band gap, along with other scintillator materials for comparison, where the experimental data are obtained from Refs. 41, 45-49, 53-56.

electron-hole pairs. Following a simple model proposed by van Roosboeck, Robbins ${ }^{50}$ derived a simple relation to determine $W: W=2.3 E_{g}$, where $E_{g}$ is the band gap energy for a given material. Using the experimental band gap energies of 10.6 and $11.8 \mathrm{eV}$ for $\mathrm{BaF}_{2}$ and $\mathrm{CaF}_{2},{ }^{51,52} \mathrm{~W}$ is thus calculated to be 24.4 and $27.1 \mathrm{eV}$, and the maximum theoretical light yields are estimated to be 40984 and $36900 \mathrm{ph} / \mathrm{MeV}$, which are close to those obtained in the present studies.

The maximum theoretical light yield as a function of band gap is shown in Fig. 7, along with those calculated for $\mathrm{NaI}$ and CsI (Refs. 5 and 11) for comparison. The additional data points allow us to determine how the achievable light yield varies with increasing band gap energy $\left(E_{\mathrm{g}}\right)$. Similarly to $\mathrm{BaF}_{2}$ and $\mathrm{CaF}_{2}$, the maximum theoretical light yields of $\mathrm{NaI}$ and $\mathrm{CsI}$ (91 743 and $83333 \mathrm{ph} / \mathrm{MeV}$, respectively) are larger than the experimentally derived absolute light yields. Most of the published light yields for pure and Tl-doped CsI range from 48000 to $76000 \mathrm{ph} / \mathrm{MeV},{ }^{48,49,53,54}$ but some larger values of 107000 and $124000 \mathrm{ph} / \mathrm{MeV}$ were also reported by Moszyński et al. ${ }^{55,56}$ for nominally pure CsI samples. For pure and Tl-doped NaI, published light yields range from 37700 to $69000 \mathrm{ph} / \mathrm{MeV} .^{41,49}$ These calculations allow the evaluation of the maximum theoretical light yield as a function of the band gap energy, and the results can be directly compared to experimental measurements, thus providing a possible pathway to validate the simulations. Also, these calculations provide insights into the possible maximum light output that can be achieved for a given scintillator material. It is of interest to note that the light yield generally decreases linearly with increasing $E_{g}$. Based on these calculations, the maximum light yield can be described by a simple linear equation

$$
L_{m}=\alpha+\beta E_{g}
$$

where $\alpha$ and $\beta$ are fitting parameters and their values are 129651.7551 and $-7761.3311 \mathrm{eV}^{-1}$, respectively.

\section{Spatial distribution of electron-hole pairs}

As noted above, a complete theory for large-scale simulations of electron-hole pair transport ${ }^{11,25}$ will require a detailed model of the spatial "nanostructure" and "microstructure" of ionization tracks. Also, the final scintillator yield strongly depends on the density of electronic excitations initially created along the track region. ${ }^{4}$ Fig. 8 shows typical spatial distributions of e-h pairs created by a $10 \mathrm{keV}$ incident photon in $\mathrm{CaF}_{2}$ and $\mathrm{BaF}_{2}$, where the electrons created by different channels are indicated by different colors. For $\mathrm{CaF}_{2}$, the interaction of the incident photon with a $\mathrm{Ca}$ atom leads to photoelectric absorption, as indicated by the arrow, resulting in the emission of a fast electron with a kinetic energy of $5.98 \mathrm{keV}$ from its $\mathrm{K}$ shell (note that the ionization energy of the $\mathrm{K}$ shell in $\mathrm{Ca}$ is $4.02 \mathrm{keV}$ ) and in the spontaneous creation of a vacancy in the same shell. The photoelectric absorption excites the corresponding $\mathrm{Ca}$ atom to a high energy state, and then the following atomic relaxation generates several low energy electrons. Further interactions of the fast electrons in $\mathrm{CaF}_{2}$ produce a number of electron-hole pairs along the track, with a large number of electron-hole pairs being created at the end of the tracks, a common feature that has been observed in other Monte Carlo simulations of energy deposition in semiconductors. ${ }^{57}$ However, the present simulations show that most e-h pairs are produced by interband transitions (from valence to conduction band) and plasmon excitation in $\mathrm{CaF}_{2}$. It should be noted that the e-h pairs created by plasmon decay are along the tracks of the primary electrons. These electrons are incapable of generating further e-h pairs as their energies are generally less than the cut-off energy. In contrast, interband transitions generally create low energy electrons with kinetic energies of about a few eV to a few $10 \mathrm{eV}$, depending on the initial kinetic energy of the scattering electron. These
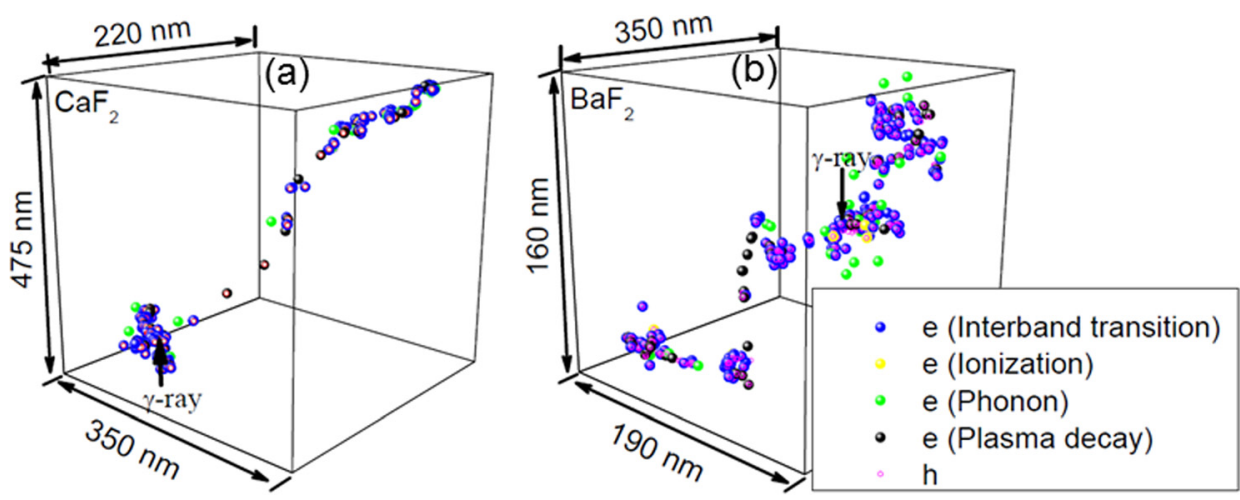

FIG. 8. Simulated spatial distribution of electron-hole pairs for a $10 \mathrm{keV}$ photon event in (a) $\mathrm{CaF}_{2}$ and (b) $\mathrm{BaF}_{2}$, where electrons and holes are distinguished by size and color, as shown in legend, and the corresponding mechanisms of creating electrons are also indicated. 

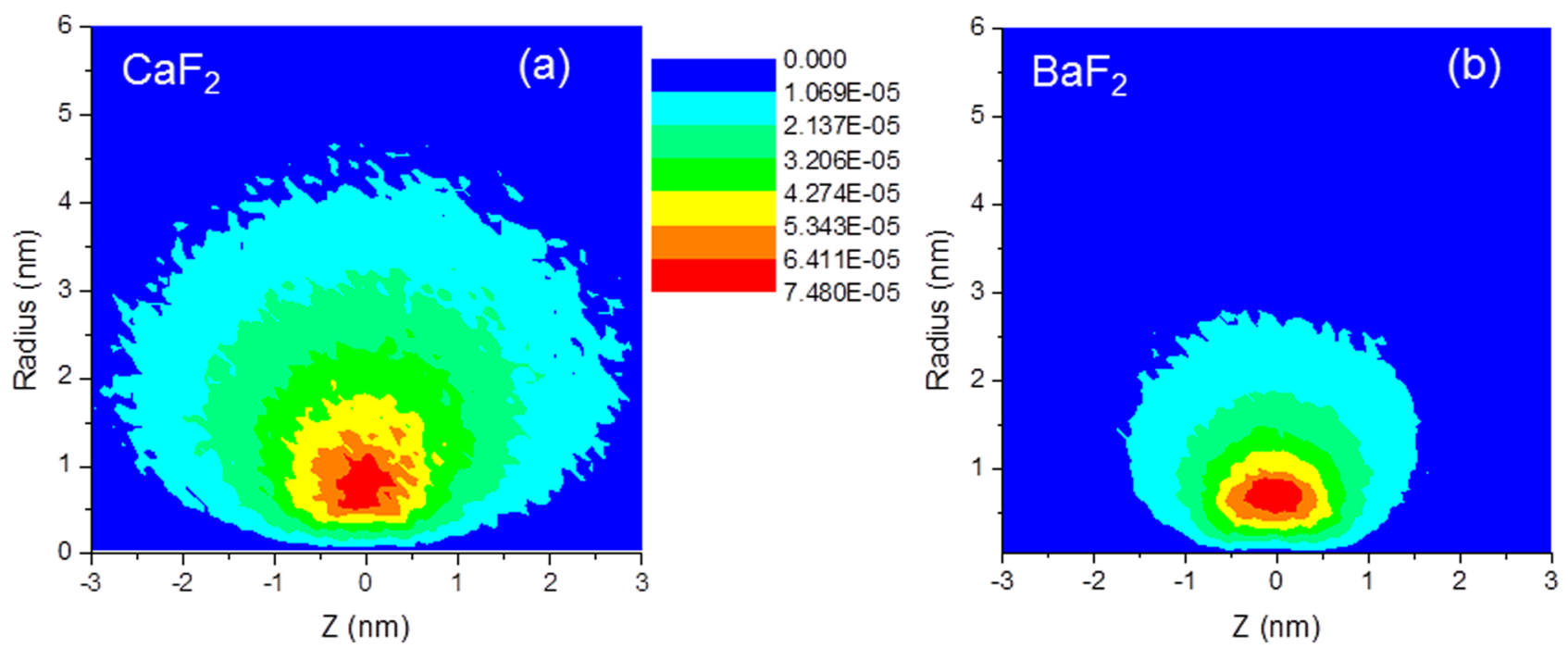

FIG. 9. Simulated density of e-h distribution averaged over $10^{4} 10 \mathrm{keV}$ incident events in (a) $\mathrm{CaF}_{2}$ and (b) $\mathrm{BaF}_{2}$.

electrons can interact with phonon, leading these electrons to move away from the primary tracks without significant loss of their energies, as indicated in Fig. 8. However, the track structures produced by the high-energy electrons are linearly distributed, except at the end of the tracks where high e-h pair density regions can form. The production of e-h pairs in $\mathrm{BaF}_{2}$ exhibits a slightly different feature to that in $\mathrm{CaF}_{2}$, as shown in Fig. 8(b), where e-h pairs are clustered, forming nanoscale domains. Therefore, the density of e-h pairs along the track in $\mathrm{BaF}_{2}$ is higher than that in $\mathrm{CaF}_{2}$, which may affect the thermalization of e-h pairs ${ }^{11,25}$ and the extent of nonlinear quenching. ${ }^{58}$ Detailed analysis of the processes taking place in the $\mathrm{BaF}_{2}$ calculation indicates that the interaction of the incident photon with a $\mathrm{Ba}$ atom leads to photoelectric absorption, as indicated by the arrow, resulting in the emission of a fast electron with a kinetic energy of $9.78 \mathrm{keV}$ from its $\mathrm{O}_{3}$ shell (note that the ionization energy of the $\mathrm{O}_{3}$ shell in $\mathrm{Ba}$ is $22 \mathrm{eV}$ ). Further interactions of the fast electron in $\mathrm{BaF}_{2}$ produce other fast electrons, thus forming several energy tracks along the fast electron track. However, it should be noted that the track structures produced by the high-energy electrons are complicated, forming several nanoscale domains with high e-h pairs densities. To better understand the ionization density distribution of e-h pairs, the spatial distribution is examined by calculating the density of e-h distribution along the tracks, as averaged $10^{4} 10 \mathrm{keV}$ incident events (gamma-ray). For a given incident energy, all the simulated tracks are projected along the $z$ axis (the incident direction), and then the radial distribution relative to the $\mathrm{z}$ axis is determined. Fig. 9 shows the density of e-h distribution from which it can be seen that all the e-h pairs are distributed with a radius of about several nm. The track radius in $\mathrm{BaF}_{2}$ is generally smaller than in $\mathrm{CaF}_{2}$, which may be correlated to the stopping power in these materials. At the end of the energy cascade, the energy loss channel for creating more electron-hole pairs is closed. Further energy loss by hot electrons is through phonon interactions until the electrons become thermalized. $^{11,25}$ It is of interest to see how these different track structures will be affected by thermalization process. However, it is to be expected that the thermalized spatial distributions would be more diffuse than those observed at the end of the electron cascade because of the slower relaxation. Given the extent of localization of the energy deposition in the energy cascade, thermalization could play a major role in determining the rates of excited state-excited state interactions, particularly for the production of scintillation light and nonproportionality.

\section{CONCLUSIONS}

In conclusion, a Monte Carlo method has been applied to simulate the interaction of photons with two scintillators, $\mathrm{BaF}_{2}$ and $\mathrm{CaF}_{2}$, and subsequent energy deposition in electron cascades. This method has been used to study various quantum mechanical processes for energy loss of primary electrons, to determine important intrinsic properties ( $W$, Fano factor, and maximum light yield) and to obtain the spatial "nanostructure" and microstructure of e-h pair distributions in $\mathrm{BaF}_{2}$ and $\mathrm{CaF}_{2}$. $W$ generally decreases with increasing incident photon energy and tends to a constant value of 18.9 and $19.8 \mathrm{eV}$ for $\mathrm{BaF}_{2}$ and $\mathrm{CaF}_{2}$ at high energies, respectively, which are in good agreement with the available theoretical data. The Fano factor for $\mathrm{BaF}_{2}$ and $\mathrm{CaF}_{2}$ is similar, which approaches 0.2 and 0.22 , respectively. The maximum theoretical light yield calculated decreases with increasing band gap energy and can be described using a simple linear relationship for the scintillator materials considered in this work.

The spatial distribution of e-h pairs exhibits a linear distribution along the tracks, and high densities of e-h pairs are formed at the end of the tracks in $\mathrm{CaF}_{2}$. The creation of e-h pairs in $\mathrm{BaF}_{2}$ shows a different feature. The track structures produced by the high-energy electrons in $\mathrm{BaF}_{2}$ are complicated, forming several nanoscale domains with high e-h pair densities. The details of the spatial distributions and densities of thermalized electron-hole pairs are important for large-scale simulations of electron-hole pair transport and to 
provide a pathway to further explore scintillation processes and performance in these materials. ${ }^{11,25}$

\section{ACKNOWLEDGMENTS}

This research was supported by the National Nuclear Security Administration, Office of Nuclear Nonproliferation Research and Development (NA-22), the US Department of Energy (DOE) at the Pacific Northwest National Laboratory, a Multiprogram National Laboratory operated by Battelle for the U.S. Department of Energy under Contract No. DEAC05-76RL01830.

${ }^{1}$ M. J. Weber, J. Lumin. 100, 35 (2002).

${ }^{2}$ E. Jaffe, D. V. Jordan, and A. J. Peurrung, Nucl. Instrum. Methods Phys. Res. A 570, 72 (2007).

${ }^{3}$ P. Dorenbos, M. Marsman, and C. W. E. Van Eijk, in Proceedings of the International Conference on Inorganic Scintillators and their Applications (Delft University Press, The Netherlands, 1996), pp. 148-155.

${ }^{4}$ S. A. Payne, N. J. Cherepy, G. Hull, J. D. Valentine, W. W. Moses, and W. S. Choong, IEEE Trans. Nucl. Sci. 56, 2506 (2009)

${ }^{5}$ F. Gao, Y. Xie, S. Kerisit, L. W. Campbell, and W. J. Weber, Nucl. Instrum. Methods Phys. Res. A 652, 564 (2011).

${ }^{6}$ A. Gibrekhterman, A. Akkerman, A. Breskin, and R. Chechik, J. Appl. Phys. 76, 1676 (1994).

${ }^{7}$ A. Akkerman, A. Gibrekhterman, A. Breskin, and R. Chechik, J. Appl. Phys. 72, 5429 (1992).

${ }^{8}$ T. Boutboul, A. Akkerman, A. Gibrekhteman, A. Breskin, and R. Chechik, J. Appl. Phys. 86, 5841 (1999).

${ }^{9}$ S. Janus and A. J. Wojtowicz, Opt. Mater. 31, 523 (2009).

${ }^{10}$ A. V. Gektin, N. Shiran, V. Nesterkina, Y. Boyarintseva, V. Baumer, G. Stryganyuk, K. Shimamura, and E. Villora, IEEE Trans. Nucl. Sci. 56, 1002 (2009).

${ }^{11}$ Z. G. Wang, Y. L. Xie, B. D. Cannon, L. W. Campbell, F. Gao, and S. Kerisit, J. Appl. Phys. 110, 064903 (2011).

${ }^{12}$ S. Kerisit, K. M. Rosso, B. D. Cannon, F. Gao, and Y. L. Xie, J. Appl. Phys. 105, 114915 (2009).

${ }^{13}$ D. E. Cullen, J. H. Hubbell, and L. Kissel, EPDL97 The Evaluated Data Library, 97 version, Report No. UCRL-50400, 1997, Vol. 6, Rev. 5.

${ }^{14}$ R. Ribberfors, Phys. Rev. A 27, 3061 (1983).

${ }^{15}$ M. J. Berger and J. H. Hubbell, "XCOM: Photon cross section on a personal computer," Report No. NBSIR 87-3597, National Bureau of Standards, Gaithersburg, MD, 1987.

${ }^{16}$ R. Mayol and F. Salvat, J. Phys. B: At. Mol. Opt. Phys. 23, 2117 (1990).

${ }^{17}$ T. A. Carlson and C. W. Nestor, Phys. Rev. A 8, 2887 (1973).

${ }^{18}$ L. W. Campbell and F. Gao, J. Lumin. 137, 121 (2013).

${ }^{19}$ X. Gonze, J. M. Beuken, R. Caracas, F. Detraux, M. Fuchs, G. M. Rignanese, L. Sindic, M. Verstraete, G. Zerah, F. Jollet, M. Torrent, A. Roy, M. Mikami, P. Ghosez, J. Y. Raty, and D. C. Allan, Comput. Mater. Sci. 25, 478 (2002).

${ }^{20}$ S. L. Adler, Phys. Rev. 126, 413 (1962)

${ }^{21}$ N. Wiser, Phys. Rev. 129, 62 (1963).

${ }^{22}$ M. S. Chung and T. E. Everhart, Phys. Rev. B 15, 4699 (1977).

${ }^{23}$ M. Dapor, Nucl. Instrum. Methods Phys. Res. B 95, 470 (1995).

${ }^{24}$ J. Llacer and E. L. Garwin, J. Appl. Phys. 40, 2766 (1969).
${ }^{25}$ Z. Wang, Y. L. Xie, L. W. Campbell, F. Gao, and S. Kerisit, J. Appl. Phys. 112, 014906 (2012).

${ }^{26}$ R. Kirkin, V. V. Mikhailin, and A. N. Vasil'ev, IEEE Trans. Nucl. Sci. 59, 2057 (2012).

${ }^{27}$ A. Kozorezov, J. K. Wigmore, and A. Owens, J. Appl. Phys. 112, 053709 (2012).

${ }^{28}$ U. Fano, Annu. Rev. Nucl. Sci. 13, 1 (1963).

${ }^{29}$ M. Inokuti, Rev. Mod. Phys. 43, 297 (1971).

${ }^{30}$ D. R. Penn, Phys. Rev. B 35, 482 (1987).

${ }^{31}$ F. Salvat, J. D. Martinez, R. Mayol, and J. Parellada, J. Phys. D 18, 299 (1985).

${ }^{32}$ J. Lindhard and M. Scharff, K. Dan. Vidensk. Selsk. Mat. Fys. Medd. 27, 15 (1953).

${ }^{33}$ J. D. Martinez, R. Mayol, and F. Salvate, J. Appl. Phys. 67, 2955 (1990).

${ }^{34}$ F. Rohrlich and B. C. Carlson, Phys. Rev. 93, 38 (1954).

${ }^{35}$ C. Møller, Ann. Phys. 406, 531 (1932).

${ }^{36}$ F. Gao, L. W. Campbell, Y. L. Xie, R. Devanathan, A. J. Perrung, and W. J. Weber, IEEE Trans. Nucl. Sci. 55, 1079 (2008).

${ }^{37}$ L. Campbell, F. Gao, R. Devanathan, and W. J. Weber, Nucl. Instrum. Methods Phys. Res. A 579, 454 (2007).

${ }^{38}$ N. J. Cherepy, G. Hull, A. D. Drobshoff, S. A. Payne, E. van Loef, C. M. Wilson, K. S. Shah, U. N. Roy, A. Burger, L. A. Boatner, W. S. Choong, and W. W. Moses, Appl. Phys. Lett. 92, 083508 (2008).

${ }^{39}$ G. W. Fraser, A. F. Abbey, A. Holland, K. McCarth, A. Owens, and A. Wells, Nucl. Instrum. Methods Phys. Res. A 350, 368 (1994).

${ }^{40}$ F. Gao, L. W. Campbell, R. Devanathan, Y. L. Xie, Y. Zhang, A. J. Peurrung, and W. J. Weber, Nucl. Instrum. Methods Phys. Res. B 255, 286 (2007).

${ }^{41}$ J. E. Jaffe, Nucl. Instrum. Methods Phys. Res. A 580, 1378 (2007).

${ }^{42}$ B. H. Bartram and A. Lempicki, J. Lumin. 68, 225 (1996).

${ }^{43}$ P. Dorenbos, IEEE Trans. Nucl. Sci. 57, 1162 (2010).

${ }^{44}$ A. Lempicki and A. J. Wojtowicz, J. Lumin. 60\&61, 942 (1994).

${ }^{45}$ P. Dorenbos, J. T. M. De Hass, R. Visser, and C. W. E. van Eijk, IEEE Trans. Nucl. Sci. 40, 424 (1993).

${ }^{46}$ A. Lempicki, A. T. Wojtowicż, and E. Berman, Nucl. Instrum. Methods Phys. Res. A 333, 304 (1993).

${ }^{47}$ M. Biasini, D. B. Cassidy, S. H. M. Deng, H. K. M. Tanaka, and A. P. Mills, Jr., Nucl. Instrum. Methods Phys. Res. A 553, 550 (2005).

${ }^{48}$ J. T. M. de Haas, P. Dorenbos, and C. W. E. Van Eijk, Nucl. Instrum. Methods Phys. Res. A 537, 97 (2005).

${ }^{49}$ S. Sasaki, H. Tawara, K. Saito, M. Miyajima, and E. Shibamura, Jpn. J. Appl. Phys. 45, 6420 (2006).

${ }^{50}$ D. J. Robbins, J. Electrochem. Soc. 127, 2694 (1980).

${ }^{51}$ R. D. Shanon, J. Appl. Phys. 73, 348 (1993).

${ }^{52} \mathrm{~J}$. W. Hodby, in Crystal with the Fluorite Structure, edited by W. Hayes (Clarendon, Oxford, 1974), p. 24.

${ }^{53}$ C. Amsler, D. Grögler, W. Joffrain, D. Lindelöf, M. Marchesotti, P. Niederberger, H. Pruys, C. Regenfus, P. Riedler, and A. Rotondi, Nucl. Instrum. Methods Phys. Res. A 480, 494 (2002).

${ }^{54}$ J. T. M. de Haas and P. Dorenbos, IEEE Trans. Nucl. Sci. 55, 1086 (2008).

${ }^{55}$ M. Moszyńskia, M. Balcerzyk, W. Czarnacki, M. Kapusta, W. Klamra, P. Schotanus, A. Syntfeld, M. Szawlowski, and V. Kozlov, Nucl. Instrum. Methods Phys. Res. A 537, 357 (2005).

${ }^{56}$ M. Moszyński, W. Czarnacki, W. Klamra, M. Szawlowski, P. Schotanus, and M. Kapusta, Nucl. Instrum. Methods Phys. Res. A 504, 307 (2003).

${ }^{57}$ G. Anton, J. Durst, M. Firsching, J. Giersch, A. Korn, M. Mitschke, D. Niederlőhner, and F. Pfeiffer, Nucl. Instrum. Methods Phys. Res. A 563, 116 (2006).

${ }^{58}$ Z. G. Wang, R. T. Williams, J. Q. Grim, F. Gao, and S. Kerisit, physica status solidi (b) 250, 1532 (2013). 


\section{Science-Driven Candidate Search for New Scintillator}

Materials

Project Number: PL13-SciDrivScintMat-PD05

Project Team: Fei Gao, Sebastien Kerisit, Yulong Xie, Dangxin Wu, Micah P. Prange, Luke W. Campbell

Pacific Northwest

NATIONAL LABORATORY

Proudly Operated by Battelle Since 1965

\section{Motivation and Objectives}

Explore the fundamental physical processes that control and limit scintillator performance

$>$ Develop comprehensive scintillator physics models/computer codes

$>$ Explain why some scintillator materials offer better performance than others (e.g. nonproportionality)

$>$ Develop comprehensive computer codes for "in silico experiments" that yield a predictive simulation framework for evaluating radiation response

$>$ Provide a real theoretical basis to improve the performance of current scintillators

$>$ Provide a pathway to optimize current scintillators and lead to the science-driven candidate search for new scintillator materials

Technical Approach

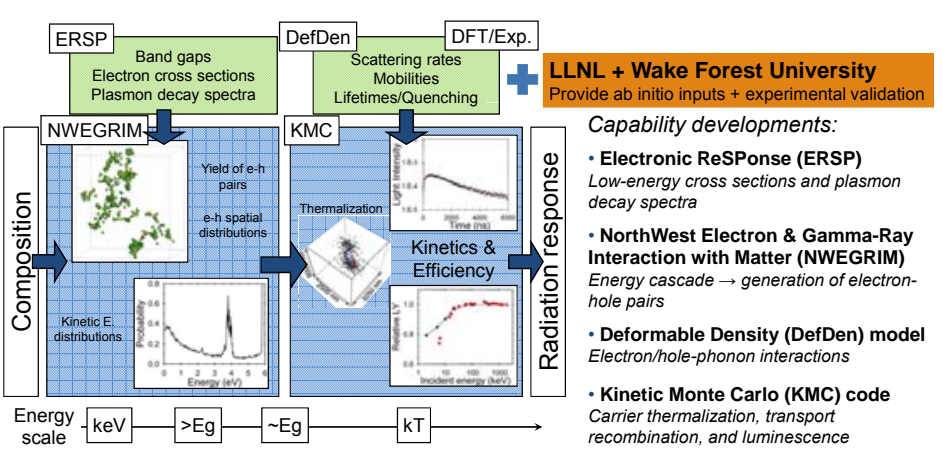

\begin{tabular}{|c|c|c|c|c|}
\hline $\begin{array}{c}\text { Capability } \\
\text { developments }\end{array}$ & $\rightleftarrows$ & $\begin{array}{c}\text { Apply \& validate model } \\
\text { Derive parameters }\end{array}$ & $\rightleftarrows$ & $\begin{array}{l}\text { Model predictions } \\
\text { In silico experiments }\end{array}$ \\
\hline
\end{tabular}

\section{Progress and Results}

$>$ Simulated a wide range of scintillators (alkali/alkaline-earth halides - $\mathrm{Nal}, \mathrm{Csl}, \mathrm{BaF}_{2}, \mathrm{CaF}_{2}, \mathrm{Srl}_{2}$; lanthanum halides - $\mathrm{LaF}_{3}, \mathrm{LaCl}_{3}, \mathrm{LaBr}_{3}$, $\mathrm{Lal}_{3}$; oxides - YAG, YAP; elpasolites - CLLC, CLLC, CLYB, CLYC), which agree with a wide range of experimental measurements and theoretical results. Evaluated light yields, decay kinetics, nonproportionality, pulse shape discrimination

$>$ Established rules that dictate scintillator performance (self-consistent analysis)

$>$ Engineered material (co-doping, defect manipulation) and search for new scintillator materials

\section{$\square$ Ab initio Calculations (Inputs for NWEGRIM)}

a $A$ b initio data model - Cross sections at E Electronic losses of heavy particles low energies of various energy loss
mechanisms and decay spectrum

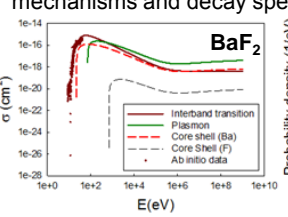

Electron cross sectio

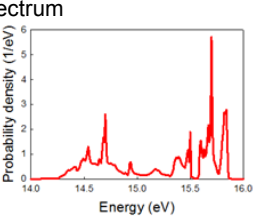

Plasmon spectrum

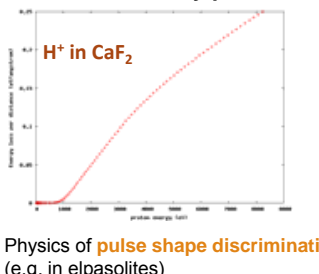

Physics of pulse sh
(e.g. in elpasolites)

\section{of elh Pairs - General Rules}

$>$ Predicted Properties

- Intrinsic properties - W vs. particle E, max. theoretical light yield, and Fano factor

- Spatial distribution of e-h pairs - track structures that are important for nonproportionality

- Energy distribution of e-h pairs - important for thermalization (nonproportionality)

Energy loss channels and stopping power

Maximum Theoretical Light Yield

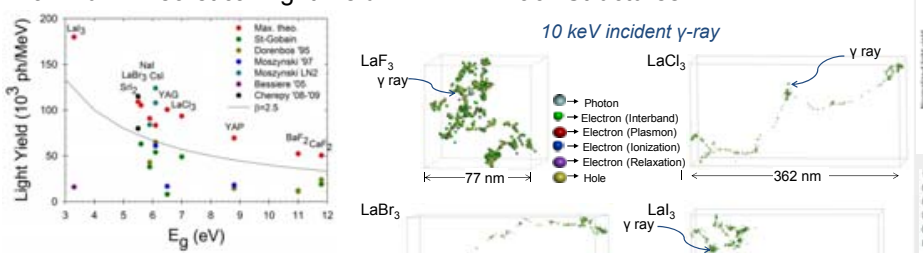

In silico experiments can be used for

rapid evaluation of:

- the potential brightness of a candidate

scintillator

the upper limit for absolute light yields $\longrightarrow \quad 354 \mathrm{~nm} \longrightarrow \quad \mathrm{k} 286 \mathrm{~nm}$ Nano-scale fluctuations in e-h pair density along the track are key to nonproportionality, where local den

General Rules for Better Scintillator Materials in silico experiments

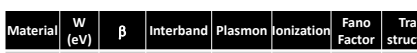

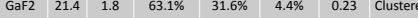
$\begin{array}{lllllllll}\text { Baf2 } & 19.1 & 1.7 & 63.7 \% & 29.3 \% & 4.1 \% & 0.19 & \text { Clustere }\end{array}$

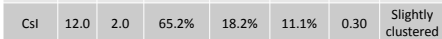
\begin{tabular}{lllllllll} 
Nal & 10.9 & 1.9 & $60.2 \%$ & $29.1 \%$ & $6.4 \%$ & 0.28 & $\begin{array}{c}\text { Slightly } \\
\text { clustered }\end{array}$ \\
\hline
\end{tabular} \begin{tabular}{l|l|l|l|l|l|l|l|l|}
\hline Labr3 & 9.5 & 1.7 & $72.1 \%$ & $21.9 \%$ & $3.2 \%$ & 0.21 & Linear \\
\hline
\end{tabular} $\begin{array}{lllllllll}\text { SrI2 } & 9.2 & 1.7 & 72.2 \% & 19.2 \% & 4.3 \% & 0.23 & \text { Liner }\end{array}$ - Small number of energy loss channels (ideally. only interband transitions) - Linear track structure that decreases nonline quenching at low energies

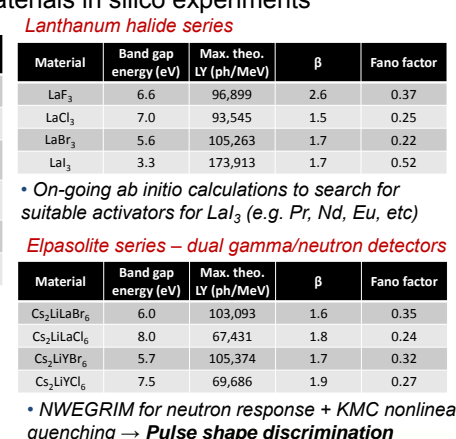

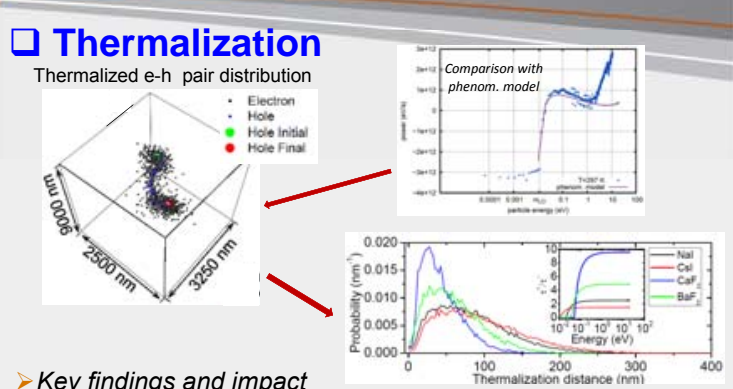

Key findings and impact

Quantified time (ps) and lengh (10's to 100's nm) scales of them Slow electron cooling significantly affects density in the track

aciltated development of improved conceptual models

$\square$ Scintillation Efficiency and Kinetics

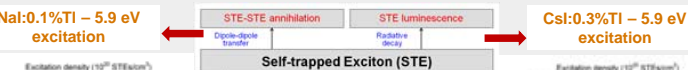
\begin{tabular}{l|l|l|l|}
\hline & Self-trapped Exciton (STE) \\
\hline
\end{tabular}

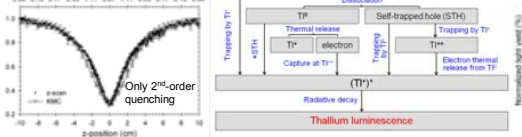

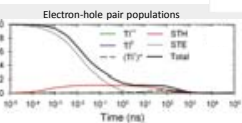
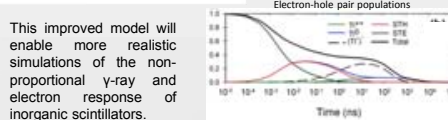

Summary and Outlook Develop in silico experiment capabilities - state-of-the-art tools Agree with a wide range of experimental measurements and other
theoretical studies. Enable accurate predictions of material modifications to increase
performance.

Provide a pathway to optimize current scintillators and perform science-

Explore the root causes of nonproportionality.

Evolve from gamma-ray to other particles and apply new capabilities to Suggest possible scintillators for crystal growth and experimental

\section{DISCOVERY}

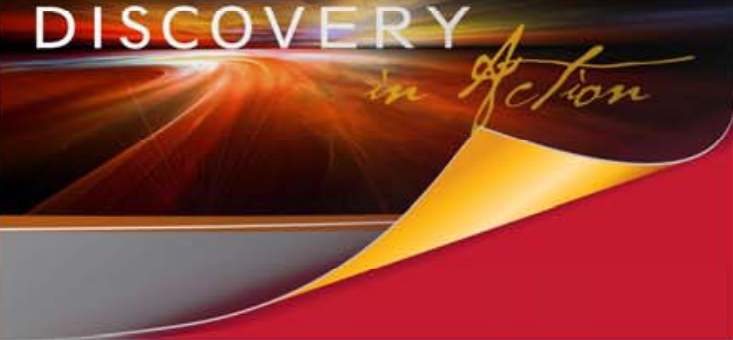




\title{
Kinetic Monte Carlo Simulations of Scintillation Processes in $\mathrm{NaI}(\mathrm{Tl})$
}

\author{
Sebastien Kerisit, Zhiguo Wang, Richard T. Williams, Member, IEEE, Joel Q. Grim, and Fei Gao
}

\begin{abstract}
Developing a comprehensive understanding of the processes that govern the scintillation behavior of inorganic scintillators provides a pathway to optimize current scintillators and allows for the science-driven search for new scintillator materials. Recent experimental data on the excitation density dependence of the light yield of inorganic scintillators presents an opportunity to incorporate parameterized interactions between excitations in scintillation models and thus enable more realistic simulations of the nonproportionality of inorganic scintillators. Therefore, a kinetic Monte Carlo (KMC) model of elementary scintillation processes in $\mathrm{NaI}(\mathrm{TI})$ is developed in this paper to simulate the kinetics of scintillation for a range of temperatures and $\mathrm{Tl}$ concentrations as well as the scintillation efficiency as a function of excitation density. The ability of the KMC model to reproduce available experimental data allows for elucidating the elementary processes that give rise to the kinetics and efficiency of scintillation observed experimentally for a range of conditions.
\end{abstract}

Index Terms-Kinetic Monte Carlo, nonproportionality, radiation detection, scintillation mechanisms, $\gamma$-ray spectroscopy.

\section{INTRODUCTION}

I NORGANIC scintillators are used extensively for radiation detection and $\gamma$-ray spectroscopy. Key properties of inorganic scintillators that determine their application as radiation detectors include their emission spectra, decay kinetics, and light yields. All of these properties are manifestations of the relaxation of ionization tracks that are created when $\gamma$-rays (or any ionizing particles) penetrate scintillator materials and produce high-energy secondary electrons that are slowed down through the creation of electron-hole pairs. Therefore, developing a thorough understanding of the elementary processes that dictate the relaxation of ionization tracks is critical to improving the performance of existing scintillator materials and accelerating the discovery of new materials. For example, the phenomenon of nonproportionality, whereby the scintillation yield normalized to the incident energy is not constant with incident energy, is

Manuscript received March 05, 2013; revised July 28, 2013, October 29, 2013, December 28, 2013, and January 10, 2014; accepted January 11, 2014 Date of publication March 06, 2014; date of current version April 10, 2014 This work was supported by the National Nuclear Security Administration, Office of Nuclear Nonproliferation Research and Engineering (NA-22), of the U.S. Department of Energy.

S. Kerisit, Z. Wang, and F. Gao are with the Physical Sciences Division, Pacific Northwest National Laboratory, Richland, WA 99352 USA (e-mail: sebastien.kerisit@pnnl.gov; zhiguo.wang@pnnl.gov; fei.gao@pnnl.gov).

R. T. Williams and J. Q. Grim are with the Department of Physics, Wake Forest University, Winston-Salem, NC 27109 USA (e-mail: williams@wfu.edu; grimjq@wfu.edu).

Digital Object Identifier 10.1109/TNS.2014.2300142 one of the main factors that degrade the energy resolution of inorganic scintillators employed in $\gamma$-ray spectroscopy; however, the underlying mechanisms that give rise to nonproportionality have not been fully elucidated and improvements in resolution have not achieved their full potential as a result.

Consequently, significant effort has been directed at identifying and quantifying the elementary processes of scintillation in inorganic materials with the aim of developing a basis for distinguishing broad trends in nonproportionality between materials classified by properties such as crystal structure, carrier mobilities, and phonon frequencies [1]-[4]. In this paper, we investigate the kinetics and efficiency of the elementary processes of scintillation in $\mathrm{NaI}(\mathrm{Tl})$. $\mathrm{NaI}$ has been widely used as a radiation detector since its discovery as a scintillation crystal in 1948 [5] and is often used as a basis for comparison for emerging scintillator materials. Over the years, several theoretical and conceptual models have been developed to explain the scintillation response of $\mathrm{NaI}[6]-[10]$.

Early modeling work by Murray and Meyer (MM) [6] focused on evaluating the scintillation efficiency of $\mathrm{NaI}$ as a function of stopping power, $\mathrm{d} E / \mathrm{d} x$, in an attempt to put the response to different ionizing particles on the same footing. The MM model assumes that self-trapped excitons (STE) are the sole energy carriers, whereas those electron-hole pairs that fail to recombine as STEs degrade the scintillation efficiency. However, Hill and Collinson [8], [11] later showed that the MM model did not give an accurate representation of the scintillation efficiency at high $\mathrm{d} E / \mathrm{d} x$. In addition, work by Dietrich et al. [12] on $\mathrm{NaI}(\mathrm{Tl})$ and $\mathrm{KI}(\mathrm{Tl})$ and by Delbecq et al. [13] on Tl-doped potassium halides, yielded proof of the presence of $\mathrm{Tl}^{0}$ and $\mathrm{Tl}^{++}$species resulting from the capture, at $\mathrm{Tl}^{+}$sites, of electrons and holes, respectively. These findings provided strong evidence that STEs were not the sole carriers. Therefore, a subsequent model was put forth by Dietrich and Murray (DM) [7], in which the creation of $\mathrm{Tl}$ excited states resulted from the binary recombination of electrons and holes (controlled either by hole diffusion or by electron de-trapping) or the prompt capture of an electron and a hole at a $\mathrm{Tl}$ site. This three-process model has been used on many occasions to model the scintillation kinetics of NaI [14], [15] and other alkali halides, (e.g., CsI [16]). A more recent model developed by Alexandrov et al. [10] extended the DM model to include the dissociation of STEs during their diffusion.

In a previous study [17], we developed a kinetic Monte Carlo (KMC) model of the scintillation mechanisms in CsI(Tl) based on the DM model. The KMC model applied a modified version of the DM model onto a 3-D lattice in which all species (activators, holes, excitons, etc.) were represented explicitly and 
treated individually. The KMC model was found to be capable of reproducing both the kinetics and the efficiency of scintillation in $\mathrm{CsI}(\mathrm{Tl})$ and, therefore, was used subsequently to simulate the nonproportionality of CsI at low incident $\gamma$-ray energies [18].

Because it does not deal with the scintillation efficiency [7], the original DM model cannot explain the variations in light yield with excitation density and, therefore, it does not allow for modeling the nonproportional behavior of alkali halides. In addition, two recent developments have provided an incentive to revisit the scintillation mechanisms of $\mathrm{NaI}$ and generate a KMC model similar to that developed for CsI. Firstly, although STEs were previously thought to diffuse faster than self-trapped holes (STH) in NaI [19]-[21], recent electronic structure calculations have provided evidence that they are equally mobile in NaI [22], [23]. Secondly, previous models of NaI scintillation did not treat potential interactions between excitations and, therefore, could not account for the excitation density dependence of the light yield. Recent experimental work [24]-[26] has made available data for parameterizing second order quenching interactions, as was done previously for CsI [27]. This approach is expected to lead to improved models of nonproportionality in inorganic scintillators.

Therefore, this paper aims to develop a KMC model of scintillation mechanisms in $\mathrm{NaI}(\mathrm{Tl})$ in order to identify the processes that give rise to its scintillation properties. Such model constitutes a building block toward a goal of simulating practical scintillator response from a moderate number of measured or calculated materials properties. An important step in building a full predictive model of scintillator response is to compare outputs of some of the component simulations to isolated, specific properties of luminescence under $\gamma$-ray excitation or intermediate experimental data measured under simpler experimental circumstances than a high-energy $\gamma$-ray track. Consequently, the present model is compared against two types of experimental data: 1) the scintillation kinetics as a function of temperature and $\mathrm{Tl}$ concentration following $\gamma$-ray excitation $(662 \mathrm{keV})$; and 2 ) the luminescence yield as a function of excitation density following ultraviolet (UV) excitation $(5.9 \mathrm{eV})$.

\section{COMPUTATIONAL APPROACH}

The KMC model was developed in a previous study of scintillation mechanisms in CsI(Tl) [17]. In this paper, it is extended to consider alkali halides that adopt the rocksalt structure (e.g., $\mathrm{NaI}$ ). In this implementation, a simple cubic lattice is used, in which each lattice point represents either a cation $\left(\mathrm{Na}^{+}\right)$or an anion $\left(\mathrm{I}^{-}\right)$and periodic boundary conditions (PBC) are applied. STHs form in alkali halides by localizing on two neighboring halide ions and can capture an electron to form STEs. Therefore, STHs and STEs are represented as occupying two neighboring $\mathrm{I}^{-}$sites. The diffusion of STHs and STEs is treated using a random-walk approach and $\mathrm{Tl}^{+}$ions are placed randomly at $\mathrm{Na}^{+}$sites to achieve the desired concentration.

The rate, $k$, of each process is determined by the Arrhenius equation: $k=A \exp \left(-W / k_{\mathrm{B}} T\right)$, where $A$ is the pre-exponential factor, $W$ the activation energy, $k_{\mathrm{B}}$ the Boltzmann constant, and $T$ the temperature. The model parameters are given in Table I and discussed in Section III.
TABLE I

PARAMETERS USED IN THE KMC MODEL

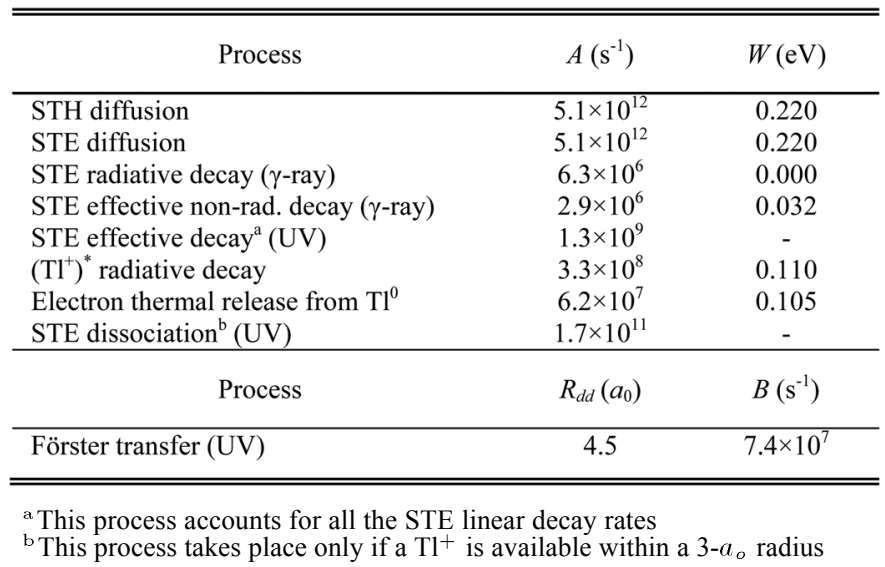

The KMC program is executed using the following algorithm: (1) the rate of each process is calculated using the Arrhenius equation and is multiplied by the number of species (STE, $\left.\mathrm{STH},\left(\mathrm{Tl}^{+}\right)^{*}, \mathrm{Tl}^{0}\right)$ that can undergo this process; (2) a process is selected with a probability proportional to the product calculated in (1) using a random number, $x_{1}$, uniform in $(0,1]$; (3) the selected process is executed on a randomly chosen species that can undergo this process; (4) time is increased by $-\ln \left(x_{2}\right) / \Gamma$, where $x_{2}$ is another random number uniform in $(0,1]$ and $\Gamma$ is the sum of all rates. Steps (1) to (4) are repeated until all electron-hole pairs have undergone radiative or nonradiative decay.

Goodness-of-fit tests were performed [28] when comparing the output of the KMC simulations with experimental data. The two-sample $\chi^{2}$ goodness-of-fit test was performed in one instance where quantitative determination of the experimental uncertainties was possible. In all other cases, statistical analysis relied on the two-sample Kolmogorov-Smirnov $(\mathrm{K}-\mathrm{S})$ test and the k-sample Anderson-Darling (A-D) test [29] instead. The $\mathrm{K}-\mathrm{S}$ test relies on the maximum deviation of the cumulative distributions of the two data sets whereas the A-D test relies on the integrated quadratic deviation of the cumulative distributions. For these three tests, the null hypothesis is that the simulated data and the experimental data were drawn from the same continuous distribution. In cases where the goodness-of-fit tests did not reject the null hypothesis, the Wald-Wolfowitz (W-W) runs test [29] was also performed [28] to evaluate whether systematic differences were present. This test relies on the number of runs in the sequence of differences between calculated and experimental data. For this test, the null hypothesis is that the sequence was produced in a random manner. The resulting p-values represent the probabilities of occurrence of the test statistics with such magnitude if the null hypothesis is true. The level of significance of the tests is 0.01 , meaning that the null hypothesis is rejected if the p-value is lower than 0.01 .

\section{Derivation of THE MOdel Parameters}

Six processes are considered in this work when simulating the $\gamma$-ray response of $\mathrm{NaI}(\mathrm{Tl}): \mathrm{STH}$ and STE diffusion, STE radiative and nonradiative decay, $\left(\mathrm{Tl}^{+}\right)^{*}$ radiative decay, and electron thermal release from $\mathrm{Tl}^{0}$. Two additional processes, namely, dipole-dipole Förster transfer and STE dissociation, are 
also considered when simulating the light yield of UV-excited NaI. A modification is also made to the STE decay rates in these simulations to account for the shortening of the STE lifetime due to quenching at the surface. Modifications made to the $\mathrm{KMC}$ model for UV-excited $\mathrm{NaI}(\mathrm{Tl})$ will be described in detail in Section IV-C.

STH and STE diffusion occurs via thermally activated hopping to near-neighbor sites. In NaI, STHs and STEs can hop in principle to eight first-nearest neighbors $\left(60^{\circ}\right.$ rotation), four second-nearest neighbors $\left(90^{\circ}\right.$ rotation), four third-nearest neighbors $\left(120^{\circ}\right.$ rotation), and two fourth-nearest neighbors $\left(180^{\circ}\right.$ rotation). Prange et al. [23] calculated the energy barriers of each of the four near-neighbor hops using density functional theory (DFT) calculations. For both STHs and STEs, they found the $60^{\circ}$ and $180^{\circ}$ rotations to have lower barriers than the $90^{\circ}$ and $120^{\circ}$ rotations. Although the calculated values differed slightly, Sadigh et al. [22] also predicted the $60^{\circ}$ and $180^{\circ}$ rotations to have lower barriers than the $90^{\circ}$ and $120^{\circ}$ rotations. Therefore, only the $60^{\circ}$ and $180^{\circ}$ hops are considered in the KMC model. Interestingly, both studies found the barriers for STHs and STEs to be similar, indicating that STEs and STHs are equally mobile in NaI. In the calculations of Prange et al. [23], the average barrier weighted by the number of possible hops for each of the two rotations considered in the KMC model was $0.2246 \mathrm{eV}$ and $0.2108 \mathrm{eV}$ for STHs and STEs, respectively. Therefore, an average energy barrier of $0.22 \mathrm{eV}$ was used for both STHs and STEs. The pre-exponential factor was derived from thermal-reorientation experiments of STHs in $\mathrm{KI}$ [30].

There is only very limited experimental data on the activation energy for STE and STH diffusion in NaI. Popp and Murray [19] determined the activation energy for STH rotation (a combination of the $60^{\circ}$ and $90^{\circ}$ rotations). Their experimental procedure involved measuring the kinetics of decay of the optical density of STHs created by either high-energy electrons or X-rays as they thermally re-orientate following bleaching at very low temperature. The temperature-dependent data yielded $0.18 \mathrm{eV}$ for the activation energy; however, the experimental fits were made for measurements taken between approximately 44 and $52 \mathrm{~K}$ suggesting that extrapolating to the temperature range of interest in this study ( 243 to $333 \mathrm{~K}$ ) may carry a sizeable uncertainty.

Nagata et al. [20] reported an activation energy for STE diffusion in $\mathrm{NaI}$ of $0.07 \mathrm{eV}$. Taken in conjunction with the activation energy for STH diffusion derived by Popp and Murray [19], this result would suggest that STEs and STHs are not equally mobile in NaI, which goes against the conclusion drawn from the calculations of Prange et al. [23] and Sadigh et al. [22]. However, the value reported by Nagata et al. was not obtained directly from their measurements. Instead, it was determined from a model fit of the change in light yield in the temperature range 5 to $200 \mathrm{~K}$, with the light yield expressed as the ratio of the radiative rate over the sum of the radiative and nonradiative rates. The radiative rate was taken to be the decay time at $5 \mathrm{~K}$ (for which the light yield is 1) and the nonradiative rate was expressed as $\left(A / T^{1 / 2}\right) \exp \left(-W / k_{\mathrm{B}} T\right)$, where $A$ and $W$ were fitted to the light yield data. Nagata et al. assumed that the nonradiative rate stemmed from the surface recombination of STEs and thus equated $W$ with the activation energy for STEs to diffuse to the surface; but additional processes could be involved, such as STE thermal nonradiative decay, quenching at surface sites, or STE dissociation. Therefore, the activation energy reported by Nagata et al. actually represents the effective activation energy of all nonradiative processes rather than the energy barrier for individual STE near-neighbor hops, which is the quantity of interest in our model.

For the STE radiative decay process, the pre-exponential factor was set to the decay time obtained by Nagata et al. [20] for pure $\mathrm{NaI}$ at $5 \mathrm{~K}$. The $4.2-\mathrm{eV}$ emission band is the dominant intrinsic emission band in pure NaI under both X-ray [31] and UV excitation [20], [32] and it is thus assumed to be the sole STE emission in the KMC model. As was done by Nagata et al., this process was taken not to be thermally activated and therefore its activation energy was set to zero. Although the parameters for the STE nonradiative decay process could be extrapolated from the data of Nagata et al. [20], these values would likely encompass other processes, as discussed above, since the data of Nagata et al. were obtained for UV excitation (near surface) and the focus of the first part of this work is on the scintillation response to $\gamma$-ray excitation. Therefore, the rate parameters for STE nonradiative decay were derived from the experimental data of Moszyński et al. [15], as described in the following paragraph

Moszyński et al. [15] measured the scintillation kinetics of $\mathrm{NaI}(\mathrm{Tl})$ following $\gamma$-ray irradiation at $662 \mathrm{keV}$ from $243 \mathrm{~K}$ to $333 \mathrm{~K}$. They fitted the scintillation decay curves with two exponential terms (termed "fast" and "slow") from $333 \mathrm{~K}$ to $273 \mathrm{~K}$ and added a third term below $273 \mathrm{~K}$ (termed "superfast"). The parameters for $\left(\mathrm{Tl}^{+}\right)^{*}$ radiative decay and for electron thermal release from $\mathrm{Tl}^{0}$ were obtained from the temperature dependence of the "fast" and "slow" components, respectively. In the $\mathrm{KMC}$ algorithm, electron release from $\mathrm{Tl}^{0}$ is assumed to lead to electron capture at $\mathrm{Tl}^{++}$. The "superfast" component could be attributed to STE emission but it has a longer decay time (160 ns at $243 \mathrm{~K}$ [15]) than expected based on the decay time of pure $\mathrm{NaI}$ obtained at a similar temperature (20 ns at $220 \mathrm{~K}$ [33]). However, the tunneling and/or de-trapping of electrons from $\mathrm{Tl}^{0}$ to recombine with STHs are likely to cause delayed STE emission [13], [34] in Tl-doped alkali halides. Therefore, the "superfast" component was taken to be due to delayed STE emission and its temperature dependence was used to obtain the parameters for STE effective nonradiative decay (i.e., accounting for the effects of electron release/tunneling from $\mathrm{Tl}^{0}$ ).

An additional factor to consider is the proportion of STEs and STHs in the KMC simulations. Thermal dissociation of STEs is likely in the conditions simulated in this work; therefore, the proportion of STEs should diminish with increasing temperature. Emkey et al. [35] determined experimentally the activation energy for exciton dissociation to be $0.06 \mathrm{eV}$. Although the first-principles calculations of Prange et al. [23] suggested a lower value $(0.02 \mathrm{eV})$ and the calculations of Sadigh et al. [22] a higher value $(0.1 \mathrm{eV})$, both values are of the same order of magnitude as the experimental value. Wang et al. [4], [36] used a phenomenological model of electron thermalization to calculate the probability of electron-hole pair recombination during thermalization and following $\gamma$-ray excitation of alkali iodide and alkaline-earth fluoride scintillators. One of the main conclusions of this work was that, although a significant proportion of electron-hole pairs rapidly recombine during thermal- 
ization, many electrons were able to travel tens to hundreds of nanometers away from the core of the track before being fully thermalized (these electrons were referred to as "stopped" electrons). The probability for recombination calculated by Wang et al. [4] at room temperature for the highest incident energy considered in that study ( 0.32 at $400 \mathrm{keV})$ was used as the reference point from which the probability of STE formation was calculated using the dissociation energy of Emkey et al. [35], as described in the Appendix. Although this approach only provides a rough estimation, which should be refined in the future, it does account for the fact that STEs are likely to thermally dissociate and, as will be shown in Section IV-A, it does lead to good agreement with the experimental data of Moszyński et al. [15]. The results were found to not be sensitive to small changes in the probability of STE formation (data not shown).

Using a simple model for describing electron capture at $\mathrm{Tl}$ sites during thermalization, Wang et al. [4], [36] were also able to evaluate the proportion of "recombined," "stopped," and "Tltrapped" electrons as a function of $\mathrm{Tl}$ concentration. As described in the Appendix, this data was used to determine the proportion of STHs and STEs as well as the number of initial electron-hole pairs in the KMC simulations as a function of $\mathrm{Tl}$ concentration. Indeed, the "stopped" electrons were considered to have travelled too far to be able to recombine with holes within the timescale of interest to this work (a few $\mu \mathrm{s}$ ) or to have trapped at defects and impurities and, therefore, they were not considered in the KMC model, which means that different initial numbers of electron-hole pairs were used as a function of $\mathrm{Tl}$ concentration.

Finally, the KMC model also considers the thermal trapping of holes, as first suggested by Hadley et al. [37] for KI(Tl) and Kaufman et al. [38] for $\mathrm{NaI}(\mathrm{Tl})$, whereby any hole created within a volume of 25 unit cells around a $\mathrm{Tl}^{+}$ion is promptly captured at that site. Therefore, a prompt capture radius, $r_{0}$, of $19 \AA$ was used in the KMC model.

\section{RESUlTS AND Discussion}

\section{A. Scintillation Kinetics as a Function of Temperature}

A series of KMC simulations were performed to calculate the scintillation kinetics of $\mathrm{NaI}$ doped with $0.1 \mathrm{~mol} \%$ of $\mathrm{Tl}$ from $243 \mathrm{~K}$ to $333 \mathrm{~K}$ for comparison with the experimental data of Moszyński et al. [15] obtained for $662-\mathrm{keV} \gamma$-rays. Because some of the parameters of the KMC model were determined based on this experimental data set, this comparison is not used to validate the KMC model. This subsection also aims to identify the processes that give rise to any changes in the observed scintillation behavior as a function of temperature.

An electron-hole pair density of $10^{18} \mathrm{~cm}^{-3}$ was used, which is within the range of densities $\left(10^{16}\right.$ to $\left.10^{19} \mathrm{~cm}^{-3}\right)$ used by Dietrich and Murray [7] based on the calculations of Katz and Kobetich [39] on the energy deposited by positive ions in NaI. The calculated scintillation decay curves are independent of the electron-hole pair density for densities lower or equal to $10^{19} \mathrm{~cm}^{-3}$. In ionization tracks created by $662-\mathrm{keV} \gamma$-rays, the proportion of regions with densities higher than $10^{19} \mathrm{~cm}^{-3}$ is expected to be small [18]. For each temperature, the scintillation decay curves were constructed from 1400 simulations, for a total of approximately 100000 electron-hole pairs. The simulation cells
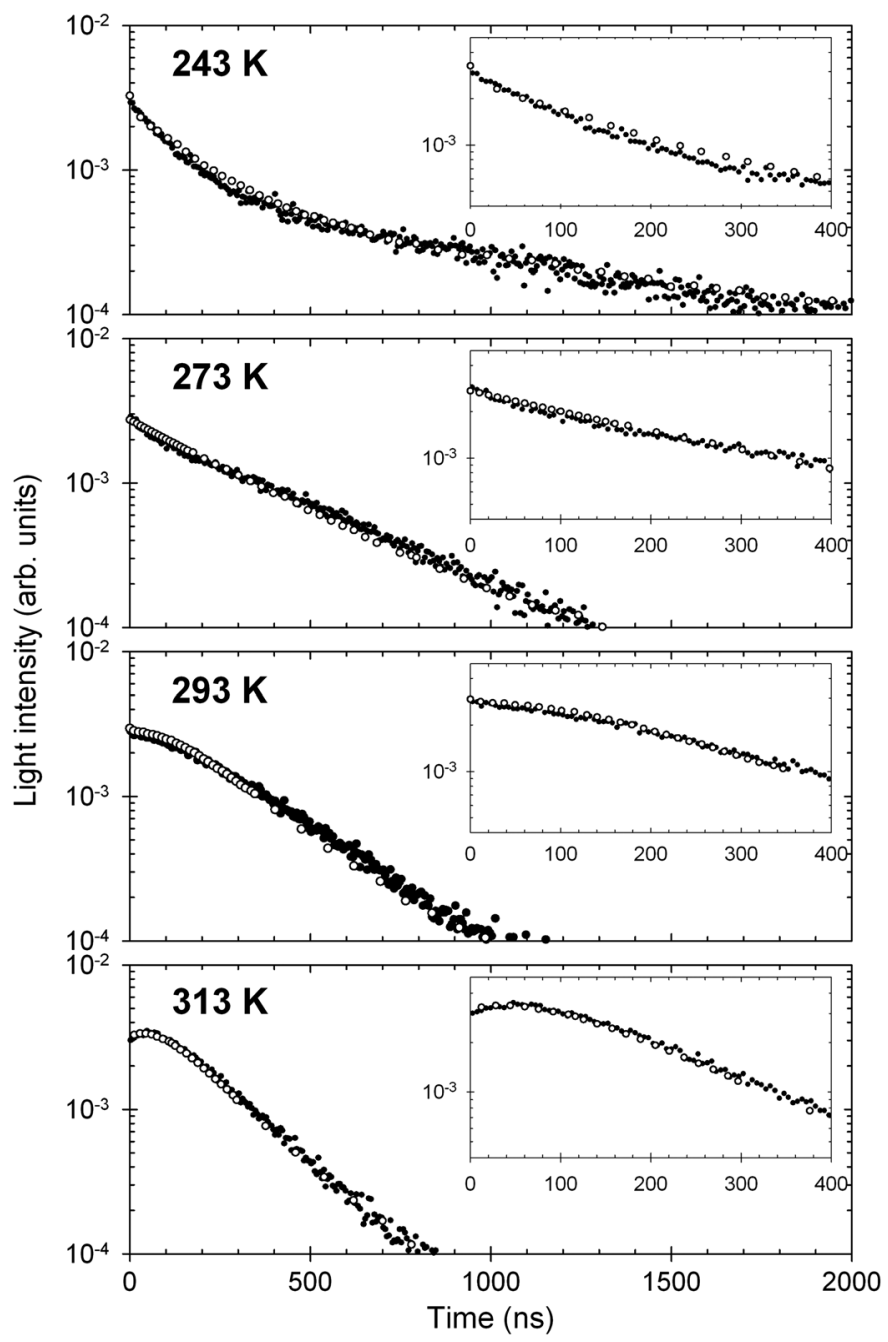

Fig. 1. Comparison of the calculated scintillation decay curves (solid circles) with the experimental data of Moszyński et al. [15] (empty circles) for a range of temperatures. Insets show a close-up of the first $400 \mathrm{~ns}$.

consisted of $128 \times 128 \times 128$ sites. Given a $\mathrm{Na}^{+}-\mathrm{I}^{-}$interatomic distance of $0.3236 \mathrm{~nm}$ [40], this corresponds to a cube with a side length of approximately $45 \mathrm{~nm}$. Activators, STHs, and STEs were placed randomly on the lattice at the start of each simulation. As was done in simulations of CsI(Tl) [17], electrons captured at $\mathrm{Tl}^{+}$sites were not positioned explicitly but, instead, a probability $x_{\mathrm{c}}$ was used, when a STH is captured at a $\mathrm{Tl}^{+}$site, to determine whether this site has previously captured an electron. This correlation probability was set to the intensity of the "fast" component determined by Moszyński et al. and serves to account for the electrostatic interactions between STHs and $\mathrm{Tl}^{0}$ sites, which are not included in the KMC model.

In Fig. 1, simulation results obtained at four temperatures are compared with the experimental data of Moszyński et al. [15]. There are small differences in a few places; in particular, the simulation results appear to systematically slightly overestimate the light intensity in the approximate time range $500-1000 \mathrm{~ns}$ for 273 and $293 \mathrm{~K}$ and, indeed, the $\mathrm{W}-\mathrm{W}$ runs test rejects the null hypothesis for these two temperatures (Table II). However, we cannot be certain of the significance of those differences because experimental error bars were not provided in [15]. In addition, the $\mathrm{K}-\mathrm{S}$ and $\mathrm{A}-\mathrm{D}$ tests did not reject the null hypothesis 
TABLE II

P-VAlues OF THE STATISTICAL TESTS OF THE COMPARISONS PRESENTED IN FIGS. 1, 4, AND 5

\begin{tabular}{lcccc}
\hline \hline Figure & Plot & $K-S^{a}$ & $A-D^{b}$ & $W-W^{c}$ \\
\hline 1 & $243 \mathrm{~K}$ & 0.844 & 0.831 & 0.065 \\
1 & $273 \mathrm{~K}$ & 1.000 & 0.992 & 0.001 \\
1 & $293 \mathrm{~K}$ & 0.979 & 0.960 & $<0.001$ \\
1 & $313 \mathrm{~K}$ & 0.994 & 0.949 & 0.618 \\
4 & $0.01 \mathrm{~mol} \%$ & 0.197 & 0.166 & 0.184 \\
4 & $0.10 \mathrm{~mol} \%$ & 0.345 & 0.146 & $<0.001$ \\
4 & $0.22 \mathrm{~mol} \%$ & 0.420 & 0.093 & $<0.001$ \\
5 & All points & 0.009 & 0.001 & - \\
5 & $<0.16 \mathrm{~mol} \%$ & $<0.001$ & 0.001 & - \\
5 & $>0.16 \mathrm{~mol} \%$ & 0.168 & 0.201 & 1.000 \\
\hline \hline
\end{tabular}

a Kolmogorov-Smirnov two-sample test

${ }^{\mathrm{b}}$ Anderson-Darling two-sample test

c Wald-Wolfowitz runs test

of equivalence of the experimental and simulated data in these cases (Table II). In other words, the simulation results are statistically consistent with the experimental data according to these two statistical tests.

Although, overall, the kinetics of scintillation accelerate with increasing temperature as expected, the experimental data show an interesting behavior in the early times. At low temperatures, $\mathrm{NaI}$ exhibits a relatively slow decay, which becomes faster with increasing temperature, but subsequently shows the appearance of an intensity maximum. Moszyński et al. concluded from this observation that $\mathrm{NaI}$ followed different mechanisms of scintillation at different temperatures.

Although only the total light intensity as a function of time can be validated against the data of Moszyński et al., it is of interest to discuss the contribution of the different processes in the $\mathrm{KMC}$ simulations in an effort to identify the mechanisms underlying this apparent change in scintillation behavior. Fig. 2 shows the contribution of several processes to the total light yield as a function of time for three temperatures. At low temperature, STE/STH diffusion is slow and STEs decay radiatively before STEs and STHs can reach $\mathrm{Tl}^{+}$sites. As the temperature increases, the STE population decays at a faster rate and STEs and STHs diffuse faster; therefore, binary recombination (i.e., sequential capture of an electron and a hole at a thallium site) becomes more dominant in the early times. Eventually, at high temperatures, the contribution of STE emission becomes small and a delayed maximum begins to appear. In summary, the rising time becomes shorter as the temperature increases but is not visible until high temperatures are reached due to the STE emission, which dominates in the early times at low temperatures.

A sensitivity analysis was carried out to determine the processes that most affect the agreement between the calculated and experimental scintillation decay curves. To do so, additional series of KMC simulations were carried out. In each series, the rate of one of the six processes $\left(k_{\mathrm{x}}\right)$ was varied between 0.1 and 10 times the rate obtained from the parameters in Table I $\left(k_{\text {norm }}\right)$. Because STH and STE diffusion use the same parameters, their rates were varied simultaneously. For each value of $k_{\mathrm{x}} / k_{\text {norm }}$, the root mean square logarithmic error was calculated from the differences between the calculated and experimental values of

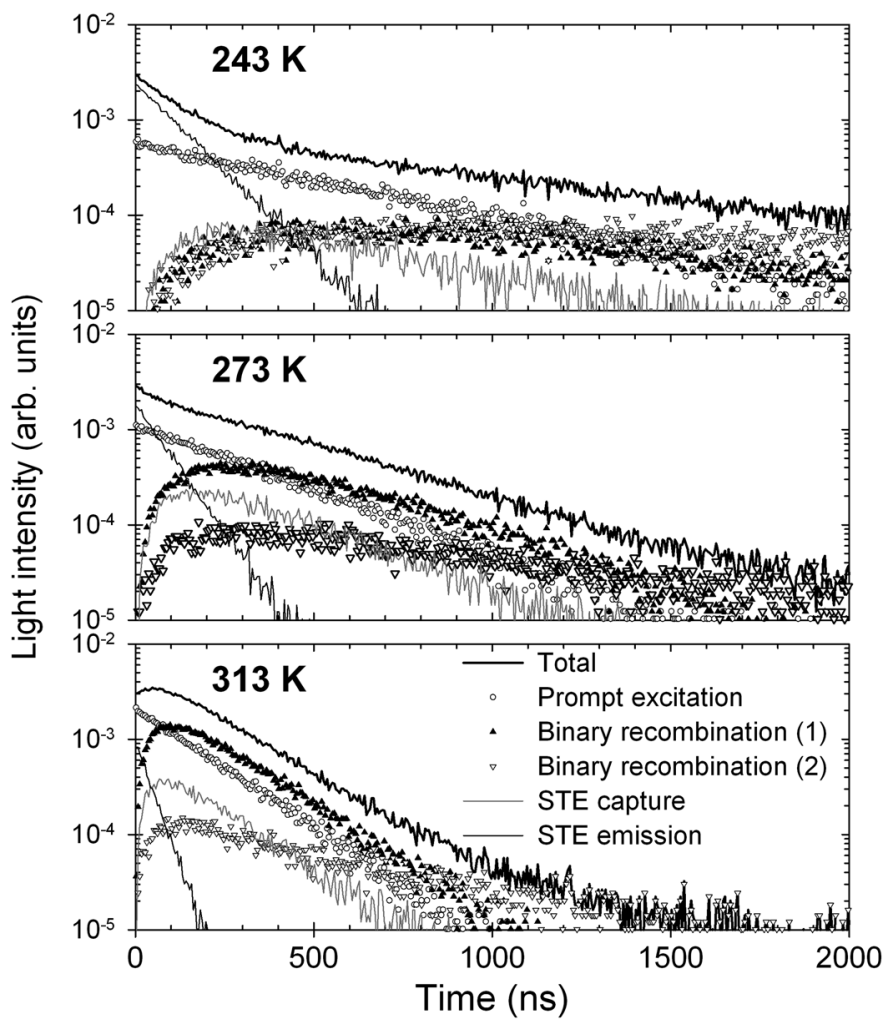

Fig. 2. Contributions from all scintillation processes to the calculated scintillation decay curves obtained at $243 \mathrm{~K}, 273 \mathrm{~K}$, and $313 \mathrm{~K}$. Binary recombination (1) is that controlled by STH diffusion whereas (2) is controlled by electron thermal release.

the light intensity for each experimental data point. Fig. 3 shows the results obtained for two of the temperatures presented in Figs. 1 and 2, and it indicates that the agreement between the calculated and experimental scintillation kinetics is most sensitive to the rate of $\left(\mathrm{Tl}^{+}\right)^{*}$ radiative decay. This is to be expected as most of the light is emitted by thallium. Fig. 3 also suggests that no significant improvement of the agreement with the data of Moszyński et al. could be obtained by changing any of the current process parameters.

\section{B. Scintillation Kinetics as a Function of Tl Concentration}

A second series of KMC simulations were performed at $298 \mathrm{~K}$ to study the scintillation kinetics of $\mathrm{NaI}(\mathrm{Tl})$ for $\mathrm{Tl}$ concentrations ranging from 0.001 to $1.0 \mathrm{~mol} \%$. The simulation cell size, number of simulations, and reference electron-hole pair density were the same as the simulations described in Section IV-A. The experimental data used for comparison in this section were not included in the model parameterization; therefore, this section provides an example of the applicability of the KMC model beyond the conditions used for its parameterization.

In Fig. 4, the simulation results are compared to the experimental data of Kubota et al. [14] obtained for $662-\mathrm{keV}$ $\gamma$-rays. In all three cases, the $\mathrm{K}-\mathrm{S}$ and $\mathrm{A}-\mathrm{D}$ tests did not reject the null hypothesis (Table II). For 0.1 and $0.22 \mathrm{~mol} \% \mathrm{Tl}$, the calculated light intensity decays slightly more slowly in the later times than the data of Kubota et al. and, indeed, the $\mathrm{W}-\mathrm{W}$ runs test rejects the null hypothesis for these two concentrations (Table II), which is indicative of systematic differences. 


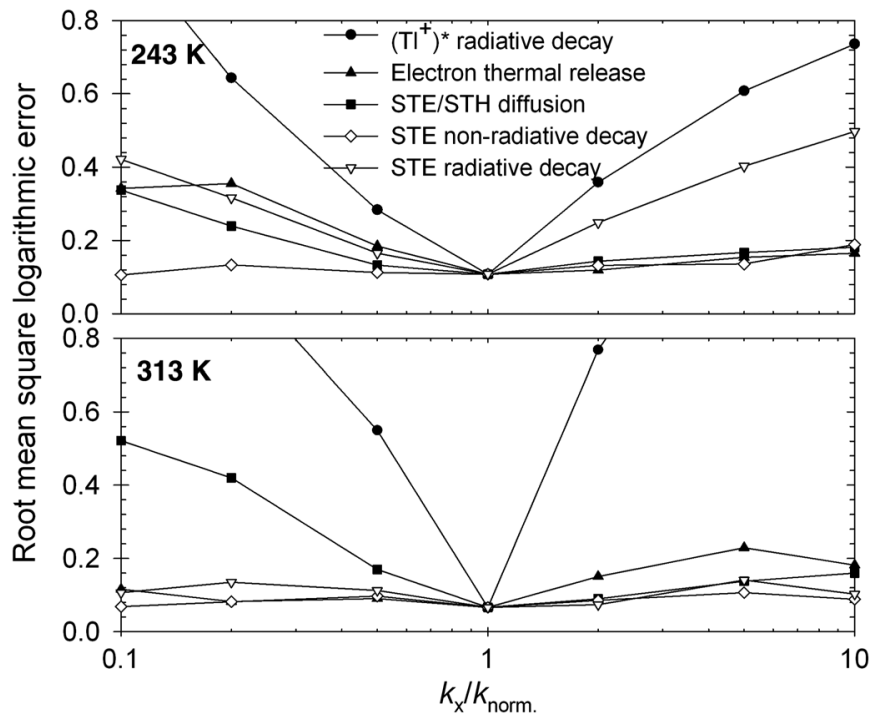

Fig. 3. Sensitivity analysis of the processes used to simulate the scintillation kinetics at 243 and $313 \mathrm{~K}$

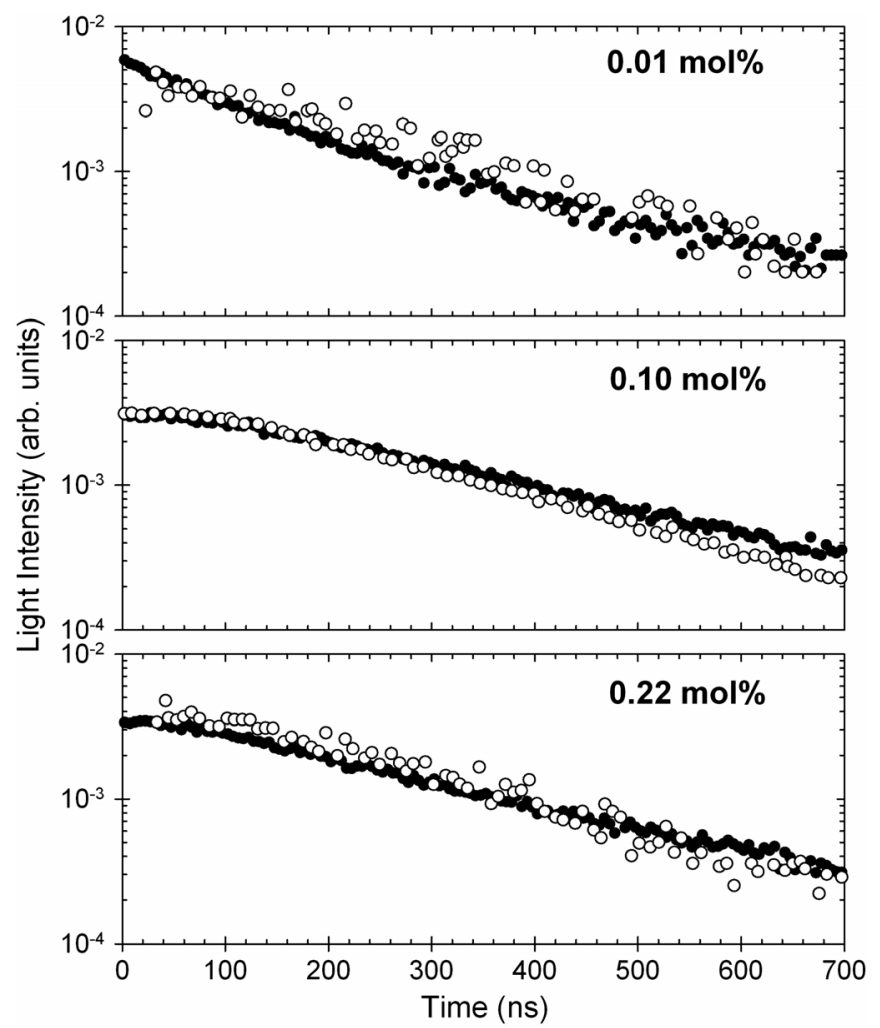

Fig. 4. Comparison of the calculated scintillation decay curves (solid circles) with the experimental data of Kubota et al. [14] (open circles-size of data points commensurate with errors) for a range of $\mathrm{Tl}$ concentrations.

Sample-to-sample variations of scintillation properties have been reported for some inorganic scintillators including, for example, the electron response of $\mathrm{NaI}(\mathrm{Tl})$ [41] and the decay time of $\mathrm{LSO}(\mathrm{Ce})[42]$. Variations can be significant for crystals grown by different manufacturers [41]. Therefore, because one of the $\mathrm{Tl}$ concentrations $(0.1 \mathrm{~mol} \%)$ was the same as that used in the comparison with the data of Moszyński et al. [15] and that, in that case, the agreement between the experimental and calculated scintillation kinetics was closer, we conclude that the

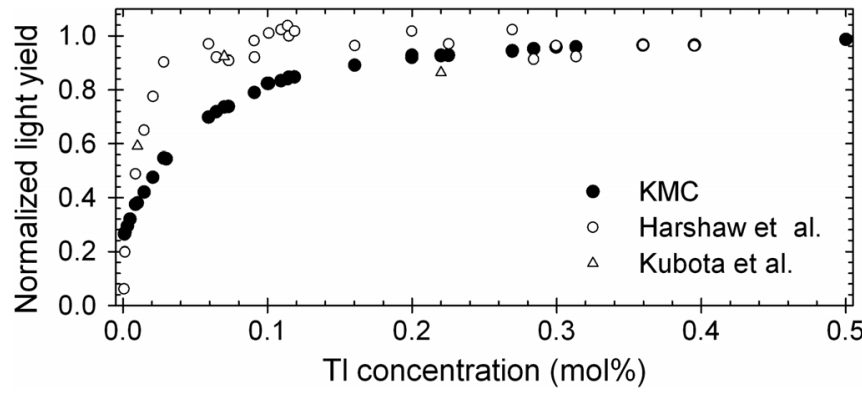

Fig. 5. Comparison of the calculated light yield with the experimental data of Harshaw et al. (as given by Murray and Meyer [6]) and Kubota et al. [44] as a function of thallium concentration.

slight discrepancy in the later times is likely due to differences in sample composition and origin and/or experimental approach used in the two experimental studies; although the possibility that the model is not fully adequate cannot be entirely ruled out.

At 0.22 and $0.01 \mathrm{~mol} \% \mathrm{Tl}$, the experimental data of Kubota et al. [14] show greater fluctuations than at $0.1 \mathrm{~mol} \%$, which makes an accurate comparison more difficult. Nonetheless, as seen for $0.1 \mathrm{~mol} \%$, the experimental data show a slightly faster decay in the later times. In the early times, the KMC simulations predict that the scintillation decay curves change from a single exponential decay to a flat top. At low $\mathrm{Tl}$ concentrations, capture of STEs and STHs at Tl sites is slow and some of the STEs are able to decay radiatively leading to the single exponential decay. As the $\mathrm{Tl}$ concentration increases, however, the STEs are captured faster and do not have time to decay radiatively and thus the flat top becomes apparent. It is difficult to compare the very early times at 0.01 and $0.22 \mathrm{~mol} \% \mathrm{Tl}$ due to the large fluctuations in the experimental data in the first few tens of nanoseconds and the lack of data points within approximately $10 \mathrm{~ns}$. Therefore, it is not possible to definitely confirm or refute this finding. However, it is consistent with the experimental observation of the appearance of an intrinsic emission peak in alkali iodide emission spectra at low $\mathrm{Tl}$ concentrations [16], [43].

From the same set of simulations, the scintillation light yield was also obtained and is compared, in Fig. 5, to the experimental data of Kubota et al. [44] and of Harshaw et al. (as given by Murray and Meyer [6]) as a function of Tl concentration. Although there is an increase in STE nonradiative decay as the Tl concentration decreases (from none at $1.0 \mathrm{~mol} \% \mathrm{Tl}$ to $10 \%$ of the electron-hole pairs at $0.001 \mathrm{~mol} \% \mathrm{Tl}$ ), the dominant factor leading to the decrease in the light yield with decreasing $\mathrm{Tl}$ concentration is the increase in the number of "stopped" electrons, which are not explicitly considered in the KMC simulations, as explained in Section III. In other words, as the Tl concentration decreases, the probability for electron capture by $\mathrm{Tl}$ during thermalization is reduced and more electrons are able to travel away from the core of the ionization track thus leading to a decrease in light output. The general trend with increasing $\mathrm{Tl}$ concentration is reproduced by the simulations; albeit with not as steep a rise as seen experimentally. The $\mathrm{K}-\mathrm{S}$ and $\mathrm{A}-\mathrm{D}$ tests reject the null hypothesis (Table II), indicating that the difference between the calculated and experimental data is statistically significant. However, parameterization of the electron capture process in the thermalization calculations performed in a previous study [36] 
was only approximate. When applying the $\mathrm{K}-\mathrm{S}$ and $\mathrm{A}-\mathrm{D}$ tests to the lower $(<0.16 \mathrm{~mol} \%)$ and higher $(>0.16 \mathrm{~mol} \%)$ ranges of Tl concentrations separately, both goodness-of-fit tests reject the null hypothesis for the lower concentration range whereas neither does for the higher range (Table II), which indicates that the simulation results are statistically consistent with the experimental data in the higher concentration range but not in the lower range.

\section{Scintillation Efficiency as a Function of Excitation Density}

A series of KMC simulations were performed at $298 \mathrm{~K}$ to investigate the effect of the initial density of electron-hole pairs on the scintillation efficiency and thus model the experiments of Grim et al. [24] on the excitation density dependence of the light yield of $\mathrm{NaI}(\mathrm{Tl})$ using sub-picosecond UV excitation (referred to hereafter as z-scan experiments). The simulation configuration was changed with respect to that used in the previous sections to reflect the changes in densities, kinetic energies, and positions relative to the surface of the electron-hole pair populations that arise due to the change in excitation energy, and thus follow the approach used previously for CsI(Tl) [27].

Eby et al. [45] reported a band gap of $5.8 \mathrm{eV}$ and a $1 \mathrm{~s}$ exciton peak at $5.56 \mathrm{eV}$ for $\mathrm{NaI}$ at $80 \mathrm{~K}$. These values are expected to decrease with increasing temperature; and, indeed, the 1s exciton peak moves to $5.39 \mathrm{eV}$ at room temperature (it was not possible to determine the band gap at room temperature in that study). Therefore, the photon energy of the UV pulse in the z-scan experiments $(5.9 \mathrm{eV})$ is slightly higher than the band gap and the 1s exciton peak and all electron-hole pairs were thus assumed to begin the simulations as STEs.

An exponentially decaying distribution: $N(z)=$ $N_{0} \exp (-\alpha z)$, where $z$ is the depth from the surface in lattice layers, $N(z)$ is the concentration of STEs at depth $z, \alpha$ is the absorption coefficient and $N_{0}$ is the concentration of STEs at the crystal surface, was used to determine the initial position of the STEs on the lattice. $N_{0}$ was calculated from the on-axis laser fluence, absorption coefficient, excitation energy, and position of the beam waist used in the z-scan experiments. The absorption coefficient was set to that used by Grim et al. [24] $\left(\alpha=4 \times 10^{5} \mathrm{~cm}^{-1}\right)$, who determined it from the optical density spectrum and film thickness reported for $\mathrm{NaI}$ by Martienssen [46]. Because of the large transverse size of the laser spot ( $\sim 17 \mu$ m radius at $1 / \mathrm{e})$ relative to the $x-y$ size of the simulation cell $(\sim 10 \mathrm{~nm})$, the transverse distribution profile was assumed to be flat.

The simulation cell was a 3-D lattice of dimensions $32 \times 32 \times$ 512 sites. Given an intersite distance of $0.3236 \mathrm{~nm}$ for NaI, this corresponds to real dimensions of $10.4 \times 10.4 \times 165.7 \mathrm{~nm}^{3}$. The UV beam was assumed to be incident along the $z$ direction and PBC were only applied in the directions perpendicular to the beam. The lattice was bound by the crystal surface at $z=0$. Although the lack of PBC at the other end of the lattice introduces an artificial boundary, the number of STEs in that region is very small given the length of the simulation cell in the $z$ direction $(165.7 \mathrm{~nm}$ ) relative to the absorption coefficient (which translates to a characteristic distance of $25 \mathrm{~nm}$ ). The STE diffusion distance is also small compared to the length of the simulation cell in the $z$ direction $(99 \%$ of the STEs diffuse over a distance of $22 \mathrm{~nm}$ or less for the lowest excitation density considered). $\mathrm{Tl}^{+}$ions were placed randomly on the lattice at $\mathrm{Na}^{+}$ sites to achieve a concentration of $0.1 \mathrm{~mol} \%$, as in the experimental sample. The light yield was determined for each excitation density using the average of 400 simulations. In addition, a few modifications were made to the model parameters relative to those used in the previous simulations.

(1) There was no prompt capture radius as all the electronhole pairs begin the simulations as STEs.

(2) Second-order STE quenching is described by dipoledipole Förster transfer, a nonradiative transfer whereby a first STE decays by emitting a virtual photon, which is absorbed by a second STE, which dissociates as a result. The rate of Förster transfer is dependent on the distance between STEs and is determined by $k(r)=B\left(R_{\mathrm{dd}} / r\right)^{6}$, where $R_{\mathrm{dd}}$ is the Förster transfer radius and $B$ is equated to the STE radiative decay rate following the formulation of Vasil'ev [47], which was used subsequently by Kirm et al. [48] and Williams et al. [26]. B was set to the inverse of the decay time (13 ns) determined by van Sciver and Bogart [33] from $\gamma$-ray excitation of pure NaI at $300 \mathrm{~K}$.

(3) STE dissociation was also considered in these simulations, whereby a STE electron, possibly in an excited bound state, may be scavenged by tunneling transfer to a nearby $\mathrm{Tl}^{+}$ site to form $\mathrm{Tl}^{0}$. The positions of the electrons at $\mathrm{Tl}^{0}$ sites were treated explicitly. Experimentally, STE dissociation was suggested from the recent time-resolved optical absorption measurements of Williams et al. [49], which showed, for CsI(Tl), that the STE absorption band decayed on the time scale of picoseconds and was replaced by a band assigned to $\mathrm{Tl}^{0}$. The same process is likely to take place in $\mathrm{NaI}$ and, since there are no direct experimental data to parameterize this process, its rate was set to that used for $\mathrm{CsI}(\mathrm{Tl})$ [27].

(4) The STE linear decay processes were modeled by a single effective decay rate, which was set to the inverse of the lifetime derived by Williams et al. [26]. Indeed, the effective lifetimes obtained by Williams et al. by fitting scintillation decay curves obtained for varying excitation densities in pure $\mathrm{NaI}$ at room temperature (singlet $=0.75 \mathrm{~ns}$, triplet $=5 \mathrm{~ns}$ ) were shorter than the radiative lifetime measured in the bulk (13 ns) by van Sciver and Bogart [33]. As suggested by Williams et al., this effect is likely due to STE quenching at the crystal surface. Therefore, an effective STE decay rate was used to implicitly account for surface quenching, which was not present in the bulk calculations carried out in the previous sections.

$R_{\text {dd }}$ was varied in increments of $a_{0} / 2$ to optimize the agreement with the experimental data. Fig. 6 compares the experimental data from the z-scan measurements of Grim et al. [24] with the calculated light yields obtained with $R_{\mathrm{dd}}=4.5 a_{0}$. The $\chi^{2}$ test rejects the null hypothesis $(\mathrm{p}-$ value $<0.0001)$, indicating that the calculated light yields are statistically different from the experimental measurements when considering the uncertainties. It is clear from Fig. 6 that there are systematic 
Excitation density $\left(10^{20} \mathrm{STEs} / \mathrm{cm}^{3}\right)$

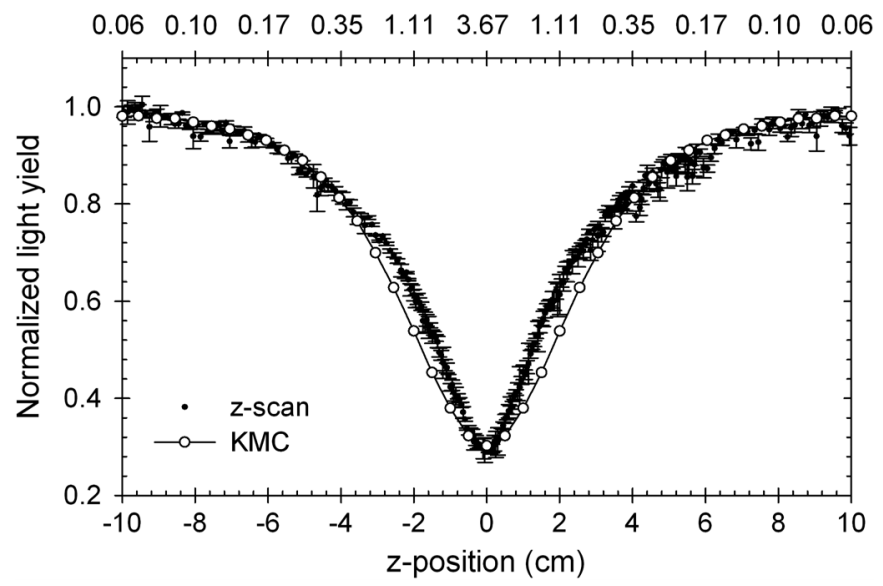

Fig. 6. Comparison of the calculated light yield (open circles) with experimental results of Grim et al. [24] (solid circles-average of 30 runs) for 5.9-eV excitation as a function of the position of the beam waist and excitation density. Experimental error bars correspond to one standard deviation. The uncertainties on the calculated light yields, as determined from the standard deviation of the mean when the simulations were divided into five independent blocks, are smaller than the data points.

differences in the interval $-4 \mathrm{~cm}<\mathrm{z}$-position $<4 \mathrm{~cm}$. The differences between observed and calculated light yields are likely due to the assumption of flat transverse profile in the KMC simulations. It should be noted that, since the experimental uncertainties were taken to be one standard deviation of the normalized light yields from 30 runs, they do not include any systematic errors that may exist on the measured light yields or the derived excitation densities. For example, although the positive and negative sides of the $\mathrm{z}$-scan should be identical, a $\chi^{2}$ test comparing the experimental light yields obtained for the two sides rejected the null hypothesis $(\mathrm{p}-$ value $<0.01)$.

The value of $R_{\mathrm{dd}}$ thus obtained corresponds to a distance of $2.91 \mathrm{~nm}$. Grim et al. [24] deduced $R_{\mathrm{dd}}$ from fitting the z-scan light yield data with a second-order rate equation model. Using an exciton capture rate of $(6 \mathrm{ps})^{-1}$ inferred from their timeresolved optical absorption measurements of $\operatorname{CsI}(0.3 \% \mathrm{Tl})$ [49] [no data are yet available for $\mathrm{NaI}(\mathrm{Tl})$ ] yielded $R_{d d}=4.1 \mathrm{~nm}$ [24]. However, if the exciton capture rate at $0.1 \% \mathrm{Tl}$ is instead deduced from linear interpolation of the measurements at $0.3 \%$ $\left[(6 \mathrm{ps})^{-1}\right]$ and $0.01 \%\left[(100 \mathrm{ps})^{-1}\right] \mathrm{Tl}, R_{\mathrm{dd}}$ becomes $2.9 \mathrm{~nm}$, in excellent agreement with the results of this work. For $\operatorname{CsI}(0.3 \%$ $\mathrm{Tl}$ ), a value of $3.66 \mathrm{~nm}$ was obtained from the KMC simulations [27], compared to $3.8 \mathrm{~nm}$ derived from the second-order rate equation model of Grim et al. [24].

In Fig. 7, the time evolution of the species populations is shown for two excitation densities. Initially, the STE population decreases due to STE emission, Förster transfer and thermal dissociation, whereby the last process leads to the formation of STHs and $\mathrm{Tl}^{0}$ sites. At low density, the $\mathrm{STH}$ and $\mathrm{Tl}^{0}$ populations plateau after a few hundred picoseconds, at which point the STH population begins to diminish as STHs are trapped at $\mathrm{Tl}^{+}$or $\mathrm{Tl}^{0}$ sites to form $\mathrm{Tl}^{++}$or $\left(\mathrm{Tl}^{+}\right)^{*}$, respectively. Subsequently, electrons are being released from $\mathrm{Tl}^{0}$ sites to form $\left(\mathrm{Tl}^{+}\right)^{*}$, which eventually emit light.

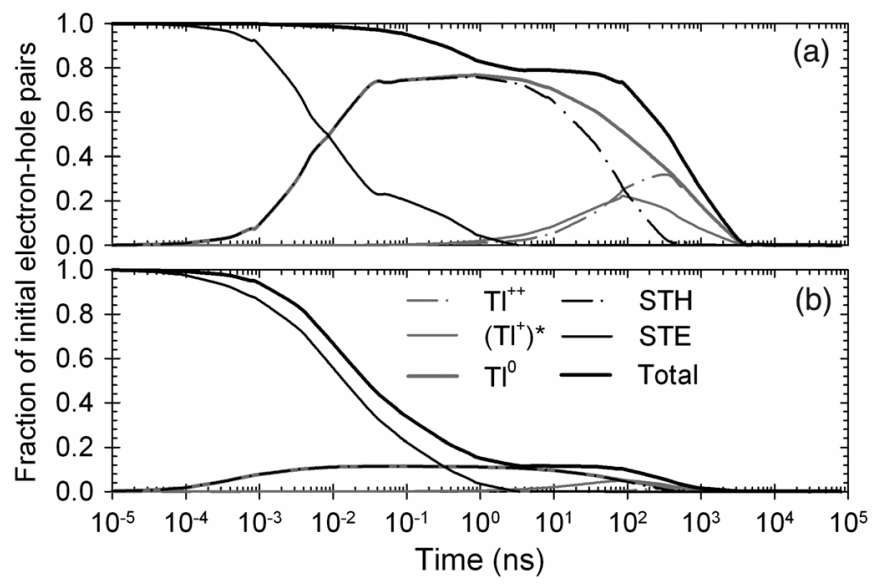

Fig. 7. Time evolution of the species considered in the KMC model at (a) $7.60 \times 10^{18} \mathrm{STEs} / \mathrm{cm}^{3}$ and (b) $3.67 \times 10^{20} \mathrm{STEs} / \mathrm{cm}^{3}$.

Fig. 7(a) also shows that all STEs disappear within 2-3 ns and the lack of any noticeable increase in the $\left(\mathrm{Tl}^{+}\right)^{*}$ population within this time frame indicates that the formation of $\left(\mathrm{Tl}^{+}\right)^{*}$ via STE capture is negligible. At high density, the sequence of events is similar. One noticeable difference is the fact that the STH and $\mathrm{Tl}^{0}$ populations plateau more rapidly and while there is still a significant population of STEs. This indicates that $\mathrm{Tl}^{+}$ sites are saturated by electrons preventing further electron scavenging. Saturation of $\mathrm{Tl}^{+}$sites by electrons means that STHs are much more likely to trap at $\mathrm{Tl}^{0}$ sites than at $\mathrm{Tl}^{+}$sites, which leads to a very small $\mathrm{Tl}^{++}$population compared to that observed at low density.

\section{CONCLUSIONS}

A kinetic Monte Carlo model was developed to simulate the scintillation response of $\mathrm{NaI}(\mathrm{Tl})$ as a function of temperature, $\mathrm{Tl}$ concentration, and excitation density. According to the purpose outlined in the Introduction, the simulations have been compared to decay times of $\gamma$-ray-excited luminescence and to photon density response data in $\mathrm{NaI}(\mathrm{Tl})$. While in several instances statistical analysis indicates that the model results reproduce the experimental data, other instances highlight areas for future improvements, such as the parameterization of the electron capture process in the thermalization calculations that were performed in a previous study [36]. This work has led to the derivation and refinement of KMC parameters, such as the Förster transfer radius for describing interactions between excitations, for future studies of scintillator performance, and the method illustrates a route for refinement of model parameters in a broad range of scintillators without the need for electron energy response or resolution measurements themselves. With such experimental validation at intermediate steps, we believe the predictive accuracy of a full Monte Carlo model of scintillator light yield and proportionality can be achieved more readily.

\section{APPENDIX}

\section{A. Estimation of the Electron Populations at $662 \mathrm{keV}$}

In our previous study of electron thermalization [4], the fractions of recombined, Tl-trapped, and stopped electrons were cal- 
culated for incident energies up to $400 \mathrm{keV}$. As they thermalize, electrons can recombine with a hole ("recombined"), become trapped at a Tl site ("Tl-trapped"), or eventually fully thermalize ("stopped"), in which case they are not considered further. The experimental data sets used for comparison in Sections IV-A and B [14], [15] were both obtained at $662 \mathrm{keV}$. Although this energy was higher than the maximum energy considered in Wang et al. [4], the fractions of recombined, Tl-trapped, and stopped electrons are linear at the highest incident energies and, therefore, a linear regression was performed using the results obtained between 20 and $400 \mathrm{keV}$ to estimate the value of each fraction at $662 \mathrm{keV}$. The values thus derived were $0.262,0.568$, and 0.171 for recombined, Tl-trapped, and stopped electrons, respectively.

\section{B. Estimation of the Extent of STE Recombination as a Function of $\mathrm{Tl}$ Concentration at $662 \mathrm{keV}$}

In Wang et al. [4], the variations in the recombined, Tl-trapped, and stopped populations were also calculated as a function of $\mathrm{Tl}$ concentration for $2-\mathrm{keV} \gamma$-rays. Therefore, the value of each population as a function of $\mathrm{Tl}$ concentration at $662 \mathrm{keV}$ was determined using the variations obtained at $2 \mathrm{keV}$ as the scaling factor

$$
f_{x}^{662}=\frac{f_{x}^{2}}{f_{0.1}^{2}} f_{0.1}^{662}
$$

where $f_{x}^{y}$ is the population (recombined, Tl-trapped, or stopped) obtained at energy $y$ and $\mathrm{Tl}$ concentration $x$. For each value of $x$, the three populations were normalized so that their sum was 1 . As mentioned above, the electrons labeled as "stopped" were not considered further. These represent electrons that have trapped at defects or impurities or that have travelled too far to be able to recombine with STHs.

Therefore, the number of electron-hole (e-h) pairs initially placed on the NaI lattice in the KMC simulations varied with $\mathrm{Tl}$ concentration. Given a number of e-h pairs $N$ that translates to $10^{18} \mathrm{e}-\mathrm{h} / \mathrm{cm}^{-3}$, the initial number of e-h pairs in the simulation of Tl concentration $x, N_{x}$, was calculated by

$$
N_{x}=N\left(f_{R}+f_{T l}\right)
$$

where $f_{R}$ and $f_{\mathrm{Tl}}$ are the fractions of recombined and Tl-trapped electrons.

The probability for an e-h pair to initially form a STE, $P_{c}$, was

$$
P_{c}=\frac{f_{R}}{\left(f_{R}+f_{T l}\right)} .
$$

The values of $f_{R}, f_{T l}, f_{S}, N_{x} / N$, and $P_{c}$ thus calculated are given in Table III.

\section{Estimation of the Extent of STE Recombination as a Function of Temperature at $662 \mathrm{keV}$}

To calculate $P_{c}$ as a function of temperature for a $\mathrm{Tl}$ concentration of $0.1 \mathrm{~mol} \%$, we write

$$
P_{c}=1-P_{d}
$$

TABLE III

$f_{R}, f_{T L}, f_{S}, N_{x} / N$, AND $P_{c}$ AS A FUnCtion of $\mathrm{T}_{\mathrm{L}}$ CONCENTRATION

\begin{tabular}{cccccc}
\hline Tl mol\% & $f_{R}$ & $f_{T l}$ & $f_{S}$ & $N_{x} / N$ & $P_{c}$ \\
\hline 0.001 & 0.290 & 0.012 & 0.697 & 0.303 & 0.959 \\
0.003 & 0.290 & 0.038 & 0.672 & 0.328 & 0.885 \\
0.005 & 0.288 & 0.061 & 0.652 & 0.348 & 0.826 \\
0.010 & 0.289 & 0.116 & 0.595 & 0.405 & 0.714 \\
0.020 & 0.284 & 0.215 & 0.501 & 0.499 & 0.570 \\
0.030 & 0.280 & 0.290 & 0.431 & 0.569 & 0.491 \\
0.050 & 0.275 & 0.406 & 0.319 & 0.681 & 0.403 \\
0.070 & 0.269 & 0.471 & 0.259 & 0.741 & 0.364 \\
0.100 & 0.262 & 0.568 & 0.171 & 0.829 & 0.316 \\
0.150 & 0.252 & 0.640 & 0.108 & 0.892 & 0.283 \\
0.200 & 0.245 & 0.680 & 0.075 & 0.925 & 0.265 \\
0.300 & 0.233 & 0.725 & 0.042 & 0.958 & 0.243 \\
0.500 & 0.219 & 0.761 & 0.020 & 0.980 & 0.223 \\
1.000 & 0.204 & 0.789 & 0.007 & 0.993 & 0.205 \\
\hline
\end{tabular}

TABLE IV

$P_{D}$ AND $P_{C}$ AS A FUnCtion OF TEMPERATURE

\begin{tabular}{ccc}
\hline $\mathrm{T}(\mathrm{K})$ & $P_{d}$ & $P_{c}$ \\
\hline 243 & 0.403 & 0.597 \\
253 & 0.452 & 0.548 \\
273 & 0.553 & 0.447 \\
293 & 0.658 & 0.342 \\
298 & 0.684 & 0.316 \\
313 & 0.766 & 0.234 \\
333 & 0.875 & 0.125 \\
\hline
\end{tabular}

where $P_{d}$ is the probability for exciton dissociation. Assuming that exciton dissociation exhibits Arrhenius behavior, we use the activation energy for exciton thermal dissociation of Emkey et al. [35] $(W=0.06 \mathrm{eV})$ to describe it

$$
P_{d}=A \exp \left(-W / k_{\mathrm{B}} T\right)
$$

where the pre-exponential factor, $A$, is obtained from the value of $P_{c}$ obtained at $T=298 \mathrm{~K}$ and given in Table III

$$
A=\exp \left(\ln \left(1-P_{c}^{298}\right)+W / 298 k_{\mathrm{B}}\right) .
$$

The resulting values of $P_{c}$ and $P_{d}$ are given in Table IV.

\section{ACKNOWLEDGMENT}

The authors are grateful to Dr. Y. L. Xie for his help with the statistical analysis. The authors also acknowledge Drs. D. Åberg, L. W. Campbell, M. Prange, B. Sadigh, and R. M. Van Ginhoven for insightful discussions.

\section{REFERENCES}

[1] Q. Li, J. Q. Grim, K. B. Ucer, A. Burger, G. A. Bizarri, W. W. Moses, and R. T. Williams, "Host structure dependence of light yield and proportionality in scintillators in terms of hot and thermalized carrier transport," Phys. Status Solidi RRL, vol. 6, pp. 346-348, Jul. 2012.

[2] R. T. Williams, J. Q. Grim, Q. Li, K. B. Ucer, G. A. Bizarri, S. Kerisit, F. Gao, P. Bhattacharya, E. Tupitsyn, E. Rowe, V. M. Buliga, and A. Burger, "Experimental and computational results on exciton/free-carrier ratio, hot/thermalized carrier diffusion, and linear/nonlinear rate constants affecting scintillator proportionality," in Proc. SPIE, Sep. 2013, vol. 8852, no. 88520J.

[3] R. Kirkin, V. V. Mikhailin, and A. N. Vasil'ev, "Recombination of correlated electron-hole pairs with account of hot capture with emission of optical phonons," IEEE Trans. Nucl. Sci., vol. 59, no. 5, pp. 2057-2064, Oct. 2012. 
[4] Z. Wang, Y.-L. Xie, L. W. Campbell, F. Gao, and S. Kerisit, "Monte Carlo simulations of electron thermalization in alkali iodide and alkaline-earth fluoride scintillators," J. Appl. Phys., vol. 112, p. 014906, Jul. 2012.

[5] R. Hofstadter, "Alkali halide scintillation counters," Phys. Rev., vol. 74, pp. 100-101, May 1948.

[6] R. B. Murray and A. Meyer, "Scintillation response of activated inorganic crystals to various charged particles," Phys. Rev., vol. 122, pp. 815-826, May 1961.

[7] H. B. Dietrich and R. B. Murray, "Kinetics of the diffusion of self-trapped holes in alkali halide scintillators," J. Lumin., vol. 5, pp. 155-170, Jul. 1972.

[8] R. Hill, "The effect on the scintillation efficiency of $\mathrm{NaI}(\mathrm{Tl})$ of changes in the thallium concentration and strain. II. Theoretical," Brit. J. Appl. Phys., vol. 17, pp. 1385-1393, Nov. 1966.

[9] J. B. West, "A theoretical model for the migration of energy from the particle track in an inorganic scintillator," J. Phys. B: Atom. Molec. Phys., vol. 3, pp. 1692-1703, Dec. 1970.

[10] B. S. Alexandrov, K. D. Ianakiev, and P. B. Littlewood, "Branching transport model of $\mathrm{NaI}(\mathrm{Tl})$ alkali-halide scintillator," Nucl. Instr. Meth. $A$, vol. 586, pp. 432-438, Mar. 2008

[11] R. Hill and A. J. L. Collinson, "The effect on the scintillation efficiency of $\mathrm{NaI}(\mathrm{Tl})$ of changes in the thallium concentration and strain. I. Experimental," Brit. J. Appl. Phys., vol. 17, pp. 1377-1383, Nov. 1966.

[12] H. B. Dietrich, A. E. Purdy, R. B. Murray, and R. T. Williams, "Kinetics of self-trapped holes in alkali-halide crystals: Experiments in $\mathrm{NaI}(\mathrm{Tl})$ and KI(Tl)," Phys. Rev. B, vol. 8, pp. 5894-5901, Dec. 1973.

[13] C. J. Delbecq, A. K. Ghosh, and P. H. Yuster, "Trapping and annihilation of electrons and positive holes in KCl-TlCl," Phys. Rev., vol. 151, pp. 599-609, Nov. 1966.

[14] S. Kubota, F. Shiraishi, and Y. Takami, "Decay curves of NaI(Tl) scintillators with different $\mathrm{Tl}^{+}$concentrations under excitation of electrons, alpha particles and fission fragments," J. Phys. Soc. Jpn., vol. 68, pp. 291-297, Jan. 1998.

[15] M. Moszyński, A. Nassalski, A. Syntfeld-Każuch, T. Szczęśniak, W. Czarnacki, D. Wolski, G. Pausch, and J. Pausch, "Temperature dependences of $\mathrm{LaBr}(\mathrm{Ce}), \mathrm{LaCl}_{3}(\mathrm{Ce})$ and $\mathrm{NaI}(\mathrm{Tl})$ scintillators," Nucl. Instr. Meth. Phys. Res. A, vol. 568, pp. 739-751, Dec. 2006.

[16] M. M. Hamada, F. E. Costa, M. C. C. Pereira, and S. Kubota, "Dependence of scintillation characteristics in the $\mathrm{CsI}(\mathrm{Tl})$ crystal on $\mathrm{Tl}^{+}$ concentrations under electron and alpha particles excitations," IEEE Trans. Nucl. Sci., vol. 48, pp. 1148-1153, Aug. 2001.

[17] S. Kerisit, K. M. Rosso, and B. D. Cannon, "Kinetic Monte Carlo model of scintillation mechanisms in CsI and CsI(T1)," IEEE Trans. Nucl. Sci., vol. 55, no. 3, pp. 1251-1258, Jun. 2008.

[18] S. Kerisit, K. M. Rosso, B. D. Cannon, F. Gao, and Y. Xie, "Computer simulation of the light yield nonlinearity of inorganic scintillators," $J$. Appl. Phys., vol. 105, p. 114915, Jun. 2009.

[19] R. D. Popp and R. B. Murray, "Diffusion of the $V_{k}$-polaron in alkali halides: Experiments in NaI and RbI," J. Phys. Chem. Solids, vol. 33, pp. 601-610, Mar. 1972.

[20] S. Nagata, K. Fujiwara, and H. Nishimura, "Dynamical aspects of excitons in NaI," J. Lumin., vol. 47, pp. 147-157, Nov.-Dec. 1991.

[21] H. Nishimura and S. Nagata, "Hopping motion of self-trapped excitons in NaI," J. Lumin., vol. 40\&41, pp. 429-430, Feb. 1988.

[22] B. Sadigh, D. Åberg, and P. Erhart, "First-principles calculations of self-trapping of carriers and excitons in $\mathrm{NaI}$ and $\mathrm{SrI}_{2}$ presented at the 2012," in Proc. IEEE Symp. Radiation Measurements and Applications, Oakland, CA, USA, May 16, 2012.

[23] M. P. Prange, R. M. Van Ginhoven, N. Govind, and F. Gao, "Formation, stability and mobility of self-trapped excitations in $\mathrm{NaI}$ and NaI(Tl)," Phys. Rev. B, vol. 87, p. 115101, Mar. 2013.

[24] J. Q. Grim, K. B. Ucer, A. Burger, P. Bhattacharya, E. Tupitsyn, E. Rowe, V. M. Buliga, L. Trefilova, A. Gektin, G. A. Bizarri, W. W. Moses, and R. T. Williams, "Nonlinear quenching of densely excited states in wide-gap solids," Phys. Rev. B, vol. 87, p. 125117, Mar. 2013.

[25] J. Q. Grim, Q. Li, K. B. Ucer, R. T. Williams, A. Burger, P. Bhattacharya, E. Tupitsyn, G. A. Bizarri, and W. W. Moses, "Nonlinear quenching rates in $\mathrm{SrI}_{2}$ and CsI scintillator hosts," MRS Proc., vol. 1341, pp. 15-20, 2011.

[26] R. T. Williams, J. Q. Grim, Q. Li, K. B. Ucer, and W. W. Moses, "Excitation density, diffusion-drift, and proportionality in scintillators," Phys. Status Solidi B, vol. 248, pp. 426-438, Feb. 2011.
[27] Z. Wang, R. T. Williams, J. Q. Grim, F. Gao, and S. Kerisit, "Kinetic Monte Carlo simulations of excitation density dependent scintillation in CsI and CsI(Tl)," Phys. Status Solidi B, vol. 250, pp. 1532-1540, Aug. 2013.

[28] "R: A language and environment for statistical computing," R Foundation for Statistical Computing. Vienna, Austria, 2013 [Online]. Available: http://www.R-project.org

[29] NIST/SEMATECH e-Handbook of Statistical Methods [Online]. Available: http://www.itl.nist.gov/div898/handbook

[30] F. J. Keller and R. B. Murray, "Thermal motion of holes in potassium iodide," Phys. Rev., vol. 150, pp. 670-679, Oct. 1966.

[31] R. B. Murray and F. J. Keller, "V ${ }_{k}$ centers and recombination luminescence in rubidium iodide and sodium iodide," Phys. Rev., vol. 153, pp. 993-1000, Jan. 1967.

[32] W. J. Van Sciver, "Fluorescence and reflection spectra of NaI single crystals," Phys. Rev., vol. 120, pp. 1193-1205, Nov. 1960.

[33] W. J. Van Sciver and L. Bogart, "Fundamental studies of scintillation phenomena in NaI," IRE Trans. Nucl. Sci., vol. 5, pp. 90-92, Dec. 1958.

[34] C. J. Delbecq, Y. Toyozawa, and P. H. Yuster, "Tunneling recombination of trapped electrons and holes in $\mathrm{KCl}: \mathrm{AgCl}$ and $\mathrm{KCl}: \mathrm{TlCl}$," Phys. Rev. B, vol. 9, pp. 4497-4505, May 1974.

[35] W. L. Emkey, A. B. Romberger, and W. J. Van Sciver, "Thermal dissociation of photoproduced excitons at the uv band edge in NaI," Phys. Rev. B, vol. 20, pp. 5326-5329, Dec. 1979.

[36] Z. Wang, Y.-L. Xie, B. D. Cannon, L. W. Campbell, F. Gao, and S. Kerisit, "Computer simulation of electron thermalization in CsI and CsI(Tl)," J. Appl. Phys., vol. 110, p. 064903, Sep. 2011.

[37] W. B. Hadley, S. Polick, R. G. Kaufman, and H. N. Hersh, "Energy storage and luminescence in KI: Tl at low temperatures," J. Chem. Phys., vol. 45, pp. 2040-2048, Sep. 1966.

[38] R. G. Kaufman, W. B. Hadley, and H. N. Hersh, "The scintillation mechanism in thallium doped alkali halides," IEEE Trans. Nucl. Sci., vol. NS-17, pp. 82-87, Apr. 1970.

[39] R. Katz and E. J. Kobetich, "Response of $\mathrm{NaI}(\mathrm{Tl})$ to energetic heavy ions," Phys. Rev., vol. 170, pp. 397-400, Jun. 1968.

[40] R. W. G. Wyckoff, Crystal Structures, 2nd Ed. ed. Hoboken, NJ, USA: New York: Interscience Publishers, 1963, vol. 1.

[41] G. Hull, W.-S. Choong, W. W. Moses, G. Bizarri, J. D. Valentine, S. A. Payne, N. J. Cherepy, and B. W. Reutter, "Measurements of $\mathrm{NaI}(\mathrm{Tl})$ electron response: Comparison of different samples," IEEE Trans. Nucl. Sci., vol. 56, no. 1, pp. 331-336, Feb. 2009.

[42] C. L. Melcher, M. Schmand, M. Eriksson, L. Eriksson, M. Casey, R. Nutt, J. L. Lefaucheur, and B. Chai, "Scintillation properties of LSO: Ce boules," IEEE Trans. Nucl. Sci., vol. 47, no. 3, pp. 965-968, Jun. 2000.

[43] M. Moszyński, M. Balcerzyk, W. Czarnacki, M. Kapusta, W. Klamra, P. Schotanus, A. Syntfeld, and M. Szawlowski, "Study of pure NaI at room and liquid nitrogen temperatures," IEEE Trans. Nucl. Sci., vol. 50, no. 4, pp. 767-773, Aug. 2003.

[44] S. Kubota, F. Shiraishi, and Y. Takami, "Scintillation process in NaI(Tl): Comparison with scintillation models," J. Phys. Soc. Jpn., vol. 68, pp. 3435-3440, Oct. 2000.

[45] J. E. Eby, K. J. Teegarden, and D. B. Dutton, "Ultraviolet absorption of alkali halides," Phys. Rev., vol. 116, pp. 1099-1105, Dec. 1959.

[46] W. Martienssen, "Uber die Excitonenbanden der Alkalihalogenid-kristalle," J. Phys. Chem. Solids, vol. 2, pp. 257-267, Oct.-Dec. 1957.

[47] A. N. Vasil'ev, "From luminescence non-linearity to scintillation nonproportionality," IEEE Trans. Nucl. Sci., vol. 55, no. 3, pp. 1054-1061, Jun. 2008.

[48] M. Kirm, V. Nagirnyi, E. Feldbach, M. De Grazia, B. Carré, G. Geoffroy, J. Gaudin, N. Fedorov, P. Martin, A. N. Vasil'ev, and A. Belsky, "Exciton-exciton interactions in $\mathrm{CdWO}_{4}$ irradiated by intense femtosecond vacuum ultraviolet pulses," Phys. Rev. B, vol. 79, p. 233103, Jun. 2009.

[49] R. T. Williams, K. B. Ucer, J. Q. Grim, K. C. Lipke, L. M. Trefilova, and W. W. Moses, "Picosecond studies of transient absorption induced by bandgap excitation of CsI and CsI: Tl at room temperature," IEEE Trans. Nucl. Sci., vol. 57, no. 3, pp. 1187-1192, Jun. 2010. 


\title{
Radiation response of inorganic scintillators: Insights from Monte Carlo simulations
}

\author{
Micah Prange, Dangxin Wu, Yulong Xie, Luke W. Campbell, Fei Gao, and Sebastien Kerisit \\ Pacific Northwest National Laboratory, Richland, WA 99352, USA
}

\begin{abstract}
The spatial and temporal scales of hot particle thermalization in inorganic scintillators are critical factors determining the extent of second- and third-order nonlinear quenching in regions with high densities of electron-hole pairs, which, in turn, leads to the light yield nonproportionality observed, to some degree, for all inorganic scintillators. Therefore, kinetic Monte Carlo simulations were performed to calculate the distances traveled by hot electrons and holes as well as the time required for the particles to reach thermal energy following $\gamma$-ray irradiation. CsI, a common scintillator from the alkali halide class of materials, was used as a model system. Two models of quasi-particle dispersion were evaluated, namely, the effective mass approximation model and a model that relied on the group velocities of electrons and holes determined from band structure calculations. Both models predicted rapid electron-hole pair recombination over short distances (a few nanometers) as well as a significant extent of charge separation between electrons and holes that did not recombine and reached thermal energy. However, the effective mass approximation model predicted much longer electron thermalization distances and times than the group velocity model. Comparison with limited experimental data suggested that the group velocity model provided more accurate predictions. Nonetheless, both models indicated that hole thermalization is faster than electron thermalization and thus is likely to be an important factor determining the extent of third-order nonlinear quenching in high-density regions. The merits of different models of quasi-particle dispersion are also discussed.
\end{abstract}

Keywords thermalization, hot electrons and holes, $\gamma$-ray spectroscopy, nonproportionality, scintillators, Monte Carlo simulations

*sebastien.kerisit@pnnl.gov; phone 1-509-371-6382; fax: 1-509-371-6354.

\section{INTRODUCTION}

Inorganic scintillators are widely used as radiation detection materials for applications ranging from high-energy physics to nuclear detection and surveillance. When used as $\gamma$-ray spectrometers, one of the most critical characteristics of inorganic scintillators is energy resolution, the ability to differentiate the energy fingerprints of incident $\gamma$-rays. One of the main sources that degrade the intrinsic energy resolution of inorganic scintillators is light yield nonproportionality. All inorganic scintillator materials exhibit some degree of nonproportionality, ${ }^{1}$ whereby the light yield normalized to the incident energy is not constant with incident energy. Although the nonproportionality of inorganic scintillators such as $\mathrm{NaI}$ and CsI was first measured decades ago, ${ }^{2}$ the underlying mechanisms of nonproportionality are not fully understood, and it is not yet possible to predict the nonproportionality of a given material based on its physicochemical properties.

Since the mid-2000s, there has been a resurgence of interest in nonproportionality ${ }^{1,3-16}$ driven in part by the need for improved resolution of inorganic scintillators to be employed in nuclear nonproliferation and homeland security applications in the United States. ${ }^{17}$ This renewed focus has led to new and significant insights into the elementary processes of nonproportionality. In particular, the importance of the thermalization of energetic electrons created during the energy cascade has recently been recognized. ${ }^{18-24}$ The scintillation process can be thought as consisting of three stages. In the first stage, the so-called energy cascade, energy is transferred to the lattice through the formation of electron-hole pairs. In the second stage, hot electrons and holes do not have sufficient energy to create further electronhole pairs and thermalize to the edges of the band gap through interactions with lattice phonons. In the third and final stage, thermalized electron-hole pairs recombine at luminescence centers to emit low-energy photons.

Hard X-Ray, Gamma-Ray, and Neutron Detector Physics XVI, edited by Arnold Burger,

Larry Franks, Ralph B. James, Michael Fiederle, Proc. of SPIE Vol. 9213, 92130L

(C) 2014 SPIE $\cdot$ CCC code: $0277-786 X / 14 / \$ 18 \cdot$ doi: $10.1117 / 12.2063818$

Proc. of SPIE Vol. 9213 92130L-1 
Theoretical predictions of the distances traveled by hot electrons during thermalization have highlighted the importance of the extent of charge separation in inorganic scintillators. ${ }^{18,22,25}$ As described by Williams and co-workers in their scintillator physical "decision tree", ${ }^{21}$ electrons and holes persist as free particles during thermalization in materials such as $\mathrm{CsI}$ and $\mathrm{NaI}$ due to their low-energy phonons. The resultant slow cooling rates, in turn, lead to third-order nonlinear quenching in regions with high electron-hole pair densities (dominant at low incident $\gamma$-ray energies) and extensive charge separation in low-density regions (dominant at high incident $\gamma$-ray energies). In materials with higher energy phonons such as oxides, faster cooling allows for the formation of excitons, which leads to second-order nonlinear quenching of varying extent depending on exciton mobility. Therefore, an accurate, quantitative determination of the thermalization distances and time scale of thermalization is critical to the development of predictive models of nonproportionality.

In previous thermalization calculations, ${ }^{18,22}$ electrons have been assumed to move according to the effective mass dispersion relation

$$
E=\frac{1}{2} m_{b} v^{2}
$$

which relates the kinetic energy, $E$, of an electron to its speed, $v$, through its effective band mass, $m_{\mathrm{b}}$. The effective mass is related to the curvature of the band structure and gives a good description of electrons near the conduction band minimum and holes near the valence band maximum, i.e. for particles with kinetic energy on the order of a fraction of one electronvolt. However, a majority of the hot electrons generated during the energy cascade have higher energies ${ }^{18,22}$ and are thus found in a variety of energy bands. Therefore, to better model the dynamics of energetic particles, a model that relates a particle speed to its energy based on the magnitude of the group velocity is considered in this work. Earlier work by Li et al. ${ }^{21}$ provided the first calculations of hot-electron group velocities in alkali and alkaline-earth iodides and put forward the existence of a connection between the magnitude of the group velocity and the range of hot electron diffusion.

In addition, previous thermalization calculations ${ }^{18,22}$ assumed that all the transferred energy could be assigned to the electron following an electron-hole pair creation event, and that holes self-trapped instantaneously on the lattice. In this work, this assumption is relaxed and the thermalization of holes is also considered in an effort to provide a quantitative estimation of the time scale during which both electrons and holes are present as free particles and to refine previous theoretical determination of the extent of charge separation. This publication focuses on CsI for several reasons: it is a widely used inorganic scintillator, it is a representative material of the category of scintillators with low-energy phonons, and we used CsI as a model system in previous work., $8,22,26,27$

\section{COMPUTATIONAL METHODS}

\subsection{Overall thermalization process}

A kinetic Monte Carlo (KMC) computer program was developed and implemented to simulate the thermalization of electrons and holes generated following $\gamma$-ray irradiation of an alkali halide lattice. The program uses as input the ionization tracks (also referred to as electron-hole pair distributions) computed with the Monte Carlo code Northwest Electron and Gamma Ray Interaction in Matter (NWEGRIM), as described in previous publications. ${ }^{28-30}$ The input data set consists of the positions, energies, and momenta of electrons and holes at the end of the energy cascade, i.e. at the point where the electrons and holes do not have sufficient energy to create further electron-hole pairs. In the thermalization program, free electrons and holes can diffuse through the alkali halide lattice while interacting with lattice phonons as well as the electric field due to all other free electrons and holes. Particles interact with both longitudinaloptical (LO) and acoustic (A) phonons leading to four possible particle-phonon interactions per particle, namely LO phonon emission, LO phonon absorption, A phonon emission, and A phonon absorption. The methodology employed to calculate the scattering rates and angles is described in section 2.3 while the approach used to compute the internal electric fields is given in section 2.4 .

The Monte Carlo program is executed using the following algorithm. 
(1) The scattering rate of each particle is determined for each of the four particle-phonon interactions.

(2) One particle-phonon interaction of one particle is selected with a probability proportional to its scattering rate

$$
\sum_{j=1}^{i-1} \frac{\tau_{j}}{\Gamma}<x_{1} \leq \sum_{j=1}^{i} \frac{\tau_{j}}{\Gamma},
$$

where $i$ is the particle-phonon interaction label, $\tau_{j}$ is the scattering rate of the $j^{\text {th }}$ process, $x_{1}$ is a random number in the range $0<x_{1} \leq 1$ determined using a random number generator, and $\Gamma$ is the sum of all scattering rates

$$
\Gamma=\sum_{j=1}^{4 N} \tau_{j}
$$

where $N$ is the total number of particles.

(3) The selected particle-phonon interaction is executed, i.e. the step length, $\delta$, and the scattering angle, $\theta$, of the selected particle are used to determine its new position and the LO or A phonon energy is used to calculate its new energy. The model used for determining phonon dispersion is described in section 2.3. The step length is obtained from the scattering rates $^{31}$

$$
\delta=\frac{-v \ln x_{2}}{\sum_{j=1}^{4} \tau_{j}},
$$

where $v$ is the particle speed and $x_{2}$ is a random number in the range $0<x_{2} \leq 1$.

(4) The new position and energy of the selected particle are used to evaluate whether it has recombined with a particle of the opposite charge or has reached thermal energy. If the energy of the particle has fallen below the thermal energy, it is considered as stopped. If either of the following two conditions are met the two particles are considered to have recombined: (a) the electrostatic interaction energy between the two particles has greater magnitude than the sum of their kinetic energies; or (b) the hole has stopped (i.e., has self-trapped) and the distance between the two particles is less than a threshold distance used to represent the finite spatial extent of a self-trapped hole. In CsI, because a self-trapped hole takes the form of an $\mathrm{I}_{2}^{-}$molecular ion, this distance is taken to be half the distance between two I ions of the $\mathrm{I}_{2}^{-}$molecular ion $(3.25 \AA)^{32}$ plus the ionic radius of $\mathrm{I}^{-}(2.06 \AA)$.

(5) Time is incremented using the following formula:

$$
\Delta t=\frac{-\ln x_{3}}{\Gamma},
$$

where $x_{3}$ is a random number in the range $0<x_{3} \leq 1$.

(6) Every $10^{-16} \mathrm{~s}$, the positions and kinetic energies of all the particles are modified due to the internal electric fields. A test is performed as in (4) to determine the fate of each particle.

Steps (1) to (6) are repeated until all particles have recombined or stopped.

\subsection{Models of quasi-particle dispersion}

Two models for describing quasi-particle dispersion are investigated in this work. The first model consists in simply using the band particle mass calculated from the band structure at the conduction band minimum for the electrons and the valence band maximum for the holes, as obtained from electronic structure calculations. This model will be referred to as the effective mass approximation (EMA) model hereafter.

In addition, polaron effects are taken into account in the effective mass approximation model using Fröhlich's theory

$$
\frac{m^{*}}{m_{b}}=1+\frac{\beta}{6},
$$

where $m^{*}$ and $m_{b}$ are the effective and band masses, respectively, and $\beta$ is given by 


$$
\beta=\frac{e}{\hbar} \frac{1}{4 \pi \varepsilon} \sqrt{\frac{m_{b}}{2 \hbar \omega_{\text {opt. }}}}\left[\frac{1}{\varepsilon_{\infty}}-\frac{1}{\varepsilon_{0}}\right],
$$

where $e$ is the elementary charge, $\hbar$ is the Planck constant, $\varepsilon$ is the vacuum permittivity, $\varepsilon_{\infty}$ and $\varepsilon_{0}$ are the optical and static dielectric constants of the material, and $\omega_{\text {opt. }}$ is the frequency of the longitudinal optical phonon.

For the second model, we propose a simple generalization of the effective mass approximation that is sensible for quasiparticles of arbitrary energy. A wave packet of energy $E$ is assumed to move with speed

$$
v(E)=\frac{\sum_{n} \int_{B Z} d^{3} k\left|\frac{d}{d \vec{k}} E_{n}(\vec{k})\right| \delta\left(E-E_{n}(\vec{k})\right)}{\sum_{n} \int_{B Z} d^{3} k \delta\left(E-E_{n}(\vec{k})\right)}
$$

in the direction of its crystal momentum, $\vec{k}$ (limited to the first Brillouin zone of the crystal). This expression reduces to the effective mass approximation near the band extrema for systems with isotropic effective mass and includes contributions from all possible quasi-particle states of energy $E$. This model will be referred to as the group velocity (GV) model hereafter. Application of these two models to CsI will be presented in the Results section.

\subsection{Electron/hole scattering rates and angles from phonons}

All the model parameters used in this work to calculate scattering rates are given in Table 1 . Table 1 only lists those parameters that were taken from experimental data or quantum mechanical calculations (primary parameters). Secondary parameters were determined from the primary parameters using the equations presented in this section.

\begin{tabular}{|c|c|c|}
\hline Parameter & Definition & Value \\
\hline$a_{0}$ & Lattice constant & $0.457 \mathrm{~nm}$ \\
\hline$\varepsilon_{\infty}$ & Optical dielectric constant ${ }^{a}$ & 3.0 \\
\hline$\varepsilon_{0}$ & Static dielectric constant ${ }^{a}$ & 5.65 \\
\hline$\hbar \omega_{\text {opt. }}$ & LO phonon energy $^{a}$ & $0.01 \mathrm{eV}$ \\
\hline$C_{11}$ & Elastic constant ${ }^{b}$ & $24.3 \mathrm{GPa}$ \\
\hline$C_{12}$ & Elastic constant ${ }^{b}$ & $6.4 \mathrm{GPa}$ \\
\hline$C_{44}$ & Elastic constant ${ }^{b}$ & $6.3 \mathrm{GPa}$ \\
\hline$\sigma$ & Integrated cross section at exciton energy & $6.7610^{-19} \mathrm{~m}^{2}$ \\
\hline$E_{\text {exc. }}$ & Exciton energy $^{c}$ & $5.3 \mathrm{eV}$ \\
\hline
\end{tabular}

Table 1. Primary model parameters used to calculate scattering rates.

${ }^{a}$ Reference 33

${ }^{b}$ Reference 34

${ }^{c}$ Reference 35

The scattering rates of electrons and holes from longitudinal-optical phonons are calculated using the Fröhlich scattering formulation, whereby the scattering rate, $\tau$, for interaction between a particle of speed $v$ and energy $E$ and a LO phonon of frequency $\omega_{\text {opt. }}$ is determined as follows:

Phonon creation

$$
\tau_{\mathrm{LO}}^{+}=\frac{1}{4 \pi \varepsilon} \frac{e^{2}}{v}\left(n_{q}+1\right) \frac{\omega_{\mathrm{opt}}}{\hbar}\left(\frac{1}{\varepsilon_{\infty}}-\frac{1}{\varepsilon_{0}}\right) \ln \left(\frac{1+A}{1-A}\right)
$$

Phonon annihilation

$$
\tau_{\mathrm{LO}}^{-}=\frac{1}{4 \pi \varepsilon} \frac{e^{2}}{v} n_{q} \frac{\omega_{\text {opt. }}}{\hbar}\left(\frac{1}{\varepsilon_{\infty}}-\frac{1}{\varepsilon_{0}}\right) \ln \left(\frac{B+1}{B-1}\right),
$$

where $A=\sqrt{1-\left(\hbar \omega_{\text {opt. }} / E\right)}, B=\sqrt{1+\left(\hbar \omega_{\text {opt. }} / E\right)}$ and $n_{\mathrm{q}}$ is the phonon occupation number: 


$$
n_{q}=\frac{1}{\exp \left(\hbar \omega_{\text {opt. }} / k_{B} T\right)-1},
$$

where $T$ is the temperature and $k_{B}$ is the Boltzmann constant.

The scattering rates of electrons and holes from acoustic phonons are calculated using the formulation of Sparks et al. ${ }^{36}$

Phonon creation

$$
\tau_{\mathrm{A}}^{+}=\frac{3 S^{2}}{4 \pi M_{p} N_{c} \hbar} \frac{1}{v} \int_{0}^{q_{\max }^{+}} d q \frac{q^{3}}{\omega(q)} \frac{1}{\left(1+\frac{q^{2}}{\alpha^{2}}\right)^{2}} f(q)\left[n_{q}+1\right] \times\left(1-\hat{k} \hat{k}^{\prime}\right)_{+}
$$

Phonon annihilation

$$
\tau_{\mathrm{A}}^{-}=\frac{3 S^{2}}{4 \pi M_{p} N_{c} \hbar} \frac{1}{v} \int_{0}^{q_{\max }^{-}} d q \frac{q^{3}}{\omega(q)} \frac{1}{\left(1+\frac{q^{2}}{\alpha^{2}}\right)^{2}} f(q) n_{q} \times\left(1-\hat{k} \hat{k}^{\prime}\right)_{-},
$$

where $q$ is the phonon momentum and the following expression is used for determining the phonon dispersion relation:

$$
\omega(q)=\left\{\begin{array}{l}
C_{s} q, q<k_{B Z} \\
C_{s} k_{B Z}, q \geq k_{B Z}
\end{array},\right.
$$

where $k_{B Z}$ is the equivalent radius of the first Brillouin zone and $C_{S}$ is the effective speed of sound in the material, which was calculated using the elastic theory

$$
\frac{3}{C_{s}}=\frac{2}{C_{t}}+\frac{1}{C_{l}},
$$

where $C_{t}$ and $C_{l}$ are the transverse and longitudinal sound velocities, respectively, which are calculated by

$$
\begin{gathered}
C_{l}=\sqrt{\frac{\frac{1}{3}\left(C_{11}+2 C_{12}+4 C_{44}\right)}{\rho}} \\
C_{t}=\sqrt{\frac{\frac{1}{3}\left(C_{11}+C_{12}+C_{44}\right)}{\rho},}
\end{gathered}
$$

where $C_{11}, C_{12}$, and $C_{44}$ are the elastic constants and $\rho$ the density of the primitive unit cell.

$M_{p}, N_{c}$, and $f(q)$ are the mass, number density, and mass correction function of the primitive cell, respectively, $S$ is the matrix element for acoustic phonon-particle interaction, and $\left(\hat{k} \hat{k}^{\prime}\right)_{ \pm}$is the cosine of the deflection angle of the particle in the collision:

$$
\left(\hat{k} \hat{k}^{\prime}\right)_{ \pm}=\frac{1-q^{2} \hbar^{2} v^{2} / 8 E^{2} \mp \hbar \omega(q) / 2 E}{\sqrt{1 \mp \hbar \omega(q) / E}} .
$$

The quantity $f(q)$, which describes the variation of the mass $M$ from the mass of the primitive cell, $M_{p}$, for small $q$, to the mass of the heaviest constituent $\left(M_{H}\right)$ for $q \geq k_{B Z}$, was calculated as proposed by Ashley et al. ${ }^{37}$ to extrapolate between the small $q$ and $q \geq k_{B Z}$ limits described by Sparks et al. ${ }^{36}$

$$
f(q)=\left\{\begin{array}{l}
1+C_{m} q^{2}, q<k_{B Z} \\
1+C_{m} k_{B Z}^{2}, q \geq k_{B Z}
\end{array},\right.
$$

where 


$$
C_{m}=\left(\frac{M_{p}}{M_{H}}-1\right) / k_{B Z}^{2}
$$

and

$$
k_{B Z}=\left(6 \pi^{2} / V_{p}\right)^{1 / 3}
$$

where $V_{p}$ is the volume of the primitive cell.

$S$ is assumed to be independent of the momentum transfer and was determined by Equation 21, as applied to $\mathrm{SiO}_{2}$ by Fischetti $^{38}$

$$
|\vec{S}(q)|^{2} \cong\left(\pi \hbar^{4} N_{c}^{2} / m^{* 2}\right) \sigma
$$

where $\sigma$ is the integrated cross section for electron scattering at the exciton energy following Sparks $e t$ al. ${ }^{36}$ and which was determined using the electron-ion scattering cross section calculated by the FEFF8 code. ${ }^{39}$

$q_{\max }^{+}$and $q_{\max }^{-}$are obtained from the conservation laws of energy and momentum:

$$
q_{\max }^{ \pm}=\left\{\begin{array}{l}
\frac{8 \pi E}{h v}\left[1 \mp C_{s} / v\right], q<k_{B Z} \\
\frac{4 \pi E}{h v}\left[1+\sqrt{1 \mp C_{S} k_{B Z} \hbar / E}\right], q \geq k_{B Z}
\end{array} .\right.
$$

The correction factor $1 /\left(1+q^{2} / \alpha^{2}\right)^{2}$ first introduced by Bradford and Woolf, ${ }^{40}$ in which $\alpha$ is the screening correction factor, was added into the integrand of the acoustic phonon-particle scattering rate described by Sparks et al. ${ }^{36}$ to correct for the fact that the calculated scattering rates become unphysical as the particle energy increases beyond the energy of the first Brillouin zone. We used the same approach used by Bradford and Woolf to determine the value of $\alpha$, i.e. by requiring that

$$
\lim _{q \rightarrow 0}\left(\frac{4 \pi}{V_{p}}\left(\frac{1}{4 \pi \varepsilon} Z_{1} Z_{2} e^{2}\right) \frac{1}{\alpha^{2}+q^{2}}\right)=S,
$$

where $Z_{1}=1$ and $Z_{2}$ is the atomic number of the dominant scattering atom (i.e. the anion for the alkali iodides).

Isotropic angular deflection is assumed for acoustic phonon-particle interactions. The scattering angle for LO phononparticle interactions is calculated as follows:

$$
\cos \theta=\left(E+E^{\prime}\right) / 2 \sqrt{E E^{\prime}}\left(1-C^{\xi}\right)+C^{\xi},
$$

where $C=\left(E+E^{\prime}+2 \sqrt{E E^{\prime}}\right) /\left(E+E^{\prime}-2 \sqrt{E E^{\prime}}\right), \xi$ is a random number in the range $0<\xi \leq 1$, and $E^{\prime}$ is the particle energy after phonon interaction:

$$
E^{\prime}=\left\{\begin{array}{l}
E-\hbar \omega_{\text {opt. }} \text { for phonon creation } \\
E+\hbar \omega_{\text {opt. }} \text { for phonon annihilation }
\end{array} .\right.
$$

The dispersion of longitudinal optical phonons was ignored and a single characteristic energy was used. The approach of Fischetti et $a l .{ }^{41}$ was employed to calculate the energy of the emitted or absorbed acoustic phonon. This approach involves inverting the probability function $P(q)$, knowing the energy $E$ of the particle that is creating or annihilating the acoustic phonon, using the rejection technique whereby

$$
P(q)=\int_{0}^{q} d q^{\prime} p\left(q^{\prime}\right) / \int_{0}^{q_{\max }^{ \pm}} d q^{\prime} p\left(q^{\prime}\right)
$$

where $q$ is the acoustic phonon wave vector and $p\left(q^{\prime}\right)$ is the content of the integral in Equations 12 and 13. Because plots of $P(q)$ as a function of $q / q_{\max }$ are very similar for all values of $E$ (whereby $E$ determines $q_{\max }$ as shown in Equation 22), a single representative value of $E$ was used to calculate the values of $P(q)$ over the interval 0 to 1 only once prior to the 
start of the simulation to save computation time. Half the maximum initial kinetic energy of a particle type was used as the single representative energy for electrons and holes.

\subsection{Internal electric fields}

The motion of free electrons and holes under the influence of internal electric fields were calculated using the classical equations of dynamics

$$
\frac{d}{d t}\left[m_{i}^{*} v_{i}\right]=-e E_{i}^{F}
$$

where $t$ is time, $m_{i}^{*}$ and $v_{i}$ are the effective mass and velocity of particle $i$, and $E_{i}^{F}$, the electric field at the position of particle $i$, is defined as

$$
E_{i}^{F}=\sum_{\substack{j=1 \\ j \neq i}}^{N_{t}} \frac{1}{4 \pi \varepsilon_{0} \varepsilon} \frac{q_{j}}{r_{i j}^{2}} \hat{r}_{i j},
$$

where $N_{t}$ is the total number of free particles, $q_{j}$ is the charge of particle $j, r_{i j}$ is the distance between particles $i$ and $j$, and $\hat{r}_{i j}$ is the unit vector pointing at particle $i$ from particle $j$. Equation 27 is discretized using a time step, $\Delta t$, of $10^{-16} \mathrm{~s}$, as determined previously. ${ }^{18}$ As Equation 28 breaks down for particle-particle distances on the order of the size of the ions, electrostatic interactions between like-charged particles were omitted when $r_{i j}$ was shorter than the lattice constant.

\section{RESULTS}

\subsection{CsI band structure and group velocities of electrons and holes}

The band structure of CsI was calculated using plane-wave density functional theory and the computer program ABINIT. ${ }^{42}$ The calculation made use of the local-density approximation (LDA) exchange-correlation potential. ${ }^{43}$ The plane-wave cutoff energy was set to 20 Hartree and a $16 \times 16 \times 16 \mathrm{k}$-point mesh was used to determine the band structure. Figure 1 shows the computed energy bands in CsI. Also shown in Figure 1 are the results of the effective mass approximation based on the curvature of the band structure near minima for electrons and maxima for holes. While the band gap is underestimated in this LDA calculation, we expect the band curvatures to be much more accurate. Figure 1 demonstrates that the agreement between the effective mass approximation and the band structure is good only near the conduction band minimum for electrons, which is the $\Gamma$-point in the plotted case, and the valence band maximum for holes, which occurs at $\mathrm{M}$ in CsI. The band masses thus obtained were $0.31 m_{0}$ and $2.27 m_{0}$ for holes and electrons, respectively. The calculated band masses are in good agreement with those calculated by Setyawan et al. ${ }^{10}$ using the generalized gradient approximation of Perdew-Burke-Enzerhof. ${ }^{44,}{ }^{45}$ These masses were used to parameterize the effective mass approximation model.
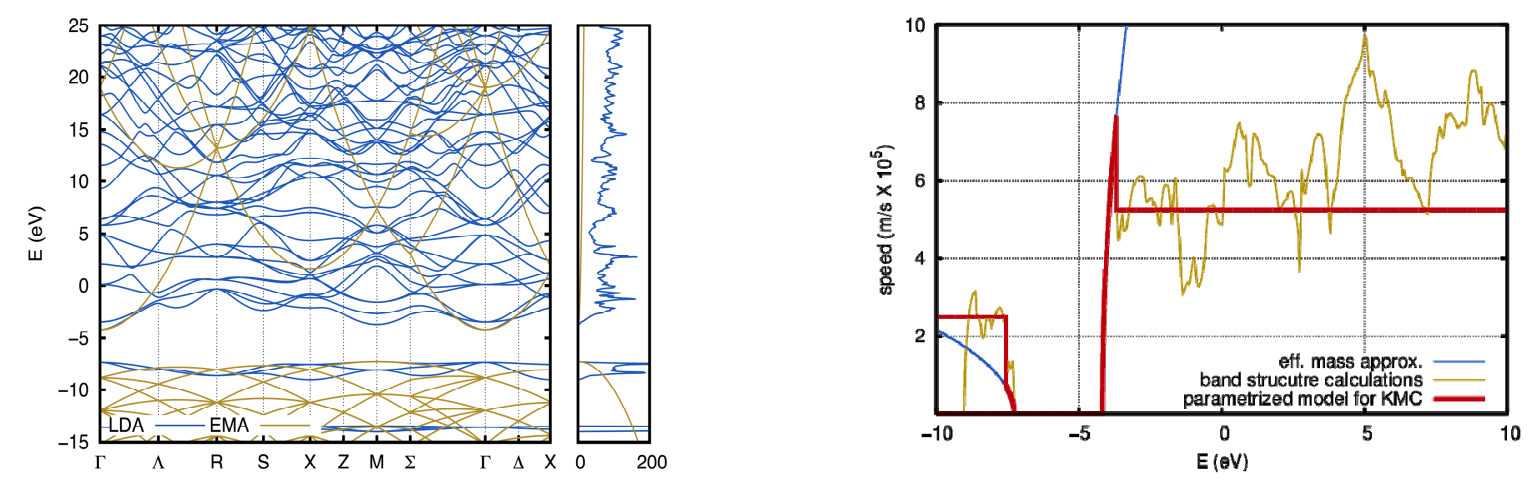

Figure 1. Computed band structure and density of states in CsI compared to the effective mass approximation (left) and average of the magnitude of the group velocity calculated from the band structure using Equation 8 (right). Also shown are the velocities computed from the effective mass approximations and a model applicable to the KMC program. 
Figure 1 also shows the average velocities of holes and electrons calculated from the band structure using Equation 8 together with those obtained based on the effective mass approximation and Equation 1. The effective mass approximation gives a good description of quasi-particle velocities near the edges of the band gap; however, it considerably overestimates the velocities of quasi-particles at higher energies with respect to the computed group velocities given by Equation 8. The results of the average of the group velocities were used to parameterize the group velocity model. To do so, a simple curve is generated, as shown in Figure 1, whereby the effective mass approximation is used for particle energies up to 0.5 and $0.3 \mathrm{eV}$ for electrons and holes, respectively, and energy-independent velocities of $5.25 \times 10^{5}$ and $2.5 \times 10^{5} \mathrm{~m} \mathrm{~s}^{-1}$ are used above these energies for electrons and holes, respectively. Polaron effects (Equations 6 and 7) were also included, although only in the energy range for which the effective mass approximation was used. Scattering rate equations are written in terms of particle speed; however, an effective mass is still required to propagate the electron and hole trajectories under the influence of the internal electric fields (Equation 27). Therefore, in the energy range where the electron and hole velocities are energy-independent, Equation 1 is used to determine the particle's effective mass.

\subsection{2-keV incident $\gamma$-rays: Comparison of effective mass approximation and group velocity models}

For both models, a series of simulations was carried out using the electron-hole pair energy and spatial distributions at the end of the energy cascade resulting from a $2-\mathrm{keV}$ incident $\gamma$-ray in CsI, as calculated by NWEGRIM. These electronhole pair distributions are the same as those used in a previous publication. ${ }^{22} 400$ simulations with approximately 170 electron-hole pairs in each distribution were run, for a total of approximately 68000 electron-hole pairs. Because any excess energy is assigned to the electron following the creation of an electron-hole pair in the NWEGRIM calculations, the valence holes have no kinetic energy in the electron-hole pair distributions used as input for the thermalization calculations. However, as illustrated in Figure 1, the valence band in CsI has a finite width of approximately $1.25 \mathrm{eV}$. Therefore, the initial kinetic energies of the holes in the thermalization calculations were varied from 0 to $1.25 \mathrm{eV}$. The kinetic energies of the electrons were scaled accordingly to conserve a constant overall initial kinetic energy.

In the simulations carried out with the first model, effective masses of $0.48 m_{0}$ and $5.56 m_{0}$ were used for the electrons and holes, respectively, based on the band masses obtained above and the polaron effects described by Equations 6 and 7 . In these simulations, particles with low initial kinetic energies or which are located in high-density regions of the tracks are able to recombine whereas those particles that do not recombine eventually reach thermal energy. The former are referred to as recombined particles and the latter as stopped particles. Recombination is very rapid and occurs within a time period not exceeding a few tenths of a picosecond. The fraction of electron-hole pairs that recombine during this period is approximately 0.35-0.40. Distances traveled by electrons and holes before recombination are shown in Figure 2 for three different initial kinetic energies of the holes $\left(E_{h}\right)$. The distributions are similar for electrons and holes and do not vary significantly with $\mathrm{E}_{\mathrm{h}}$. Figure 2 shows that almost all the particles that recombine do so within approximately 5 $\mathrm{nm}$.
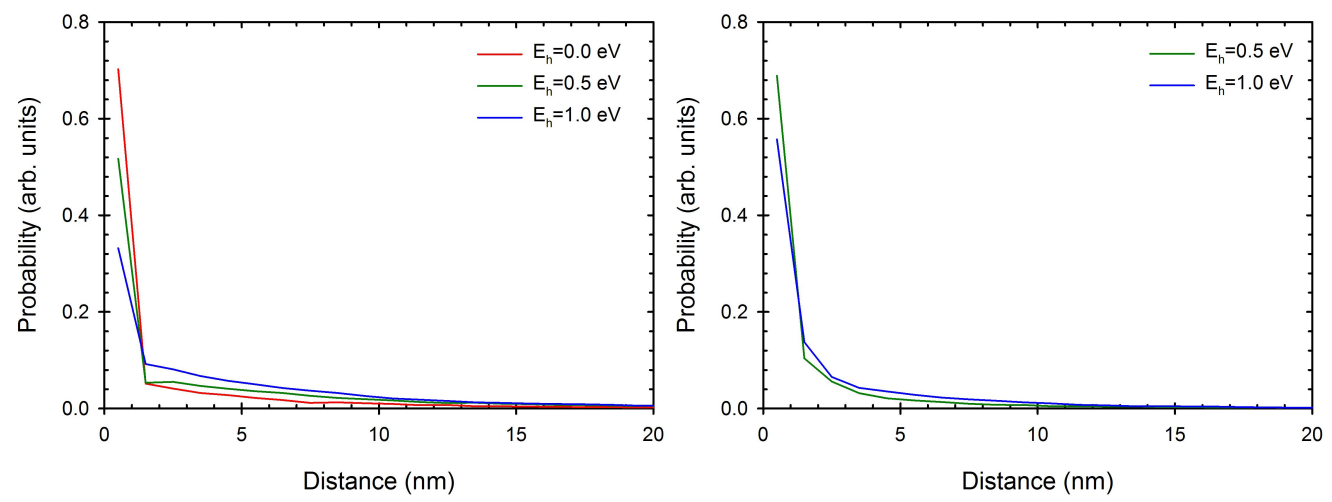

Figure 2. Effective mass approximation model. Distance distributions of recombined electrons (left) and holes (right) following irradiation of CsI with 2-keV $\gamma$-rays for three different initial kinetic energies of the holes $\left(\mathrm{E}_{\mathrm{h}}\right)$. 
Thermalization distances of stopped electrons and holes are shown in Figure 3. The thermalization distance is defined as the distance between the final (that is, at the point where the particle reaches thermal energy) and initial position of a given particle. As expected, electrons travel longer distances than holes because of their much lower effective mass. The thermalization distance distributions of the electrons show a maximum at approximately $50 \mathrm{~nm}$ with a very long tail that extends up to $3000 \mathrm{~nm}$ whereas the distance distributions of the holes peak at approximately $10 \mathrm{~nm}$ with a tail that only reaches up to $30 \mathrm{~nm}$. The effect of the initial kinetic energy of the holes is only small with a slight shift to longer distances of the distance distribution of the holes and a greater proportion of shorter distances exhibited by the distance distributions of the electrons.
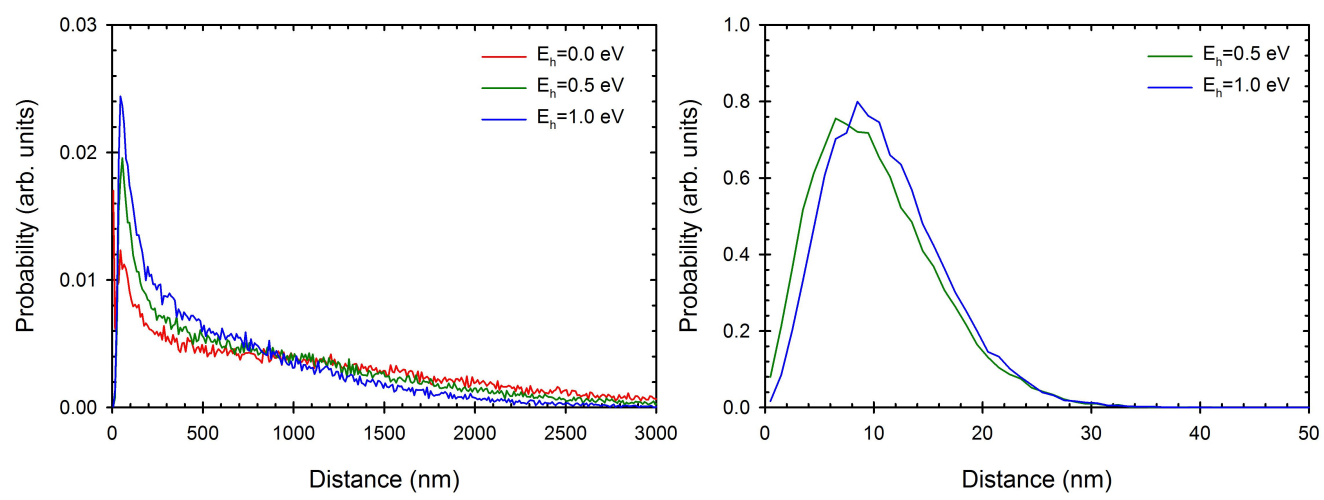

Figure 3. Effective mass approximation model. Distance distributions of stopped electrons (left) and holes (right) following irradiation of CsI with 2-keV $\gamma$-rays for three different initial kinetic energies of the holes $\left(\mathrm{E}_{\mathrm{h}}\right)$.

The thermalization time distributions of stopped electrons and holes are shown in Figure 4. The thermalization time is defined as the time required for a given particle to reach thermal energy. The thermalization time distributions show very similar shapes to the thermalization distance distributions: the time distributions of the holes peak between 0.6 and $0.8 \mathrm{ps}$ and only extend up to $1.5 \mathrm{ps}$ whereas those of the electrons peak at a fraction of a picosecond and can reach up to 15 to 25 ps depending on $E_{h}$.
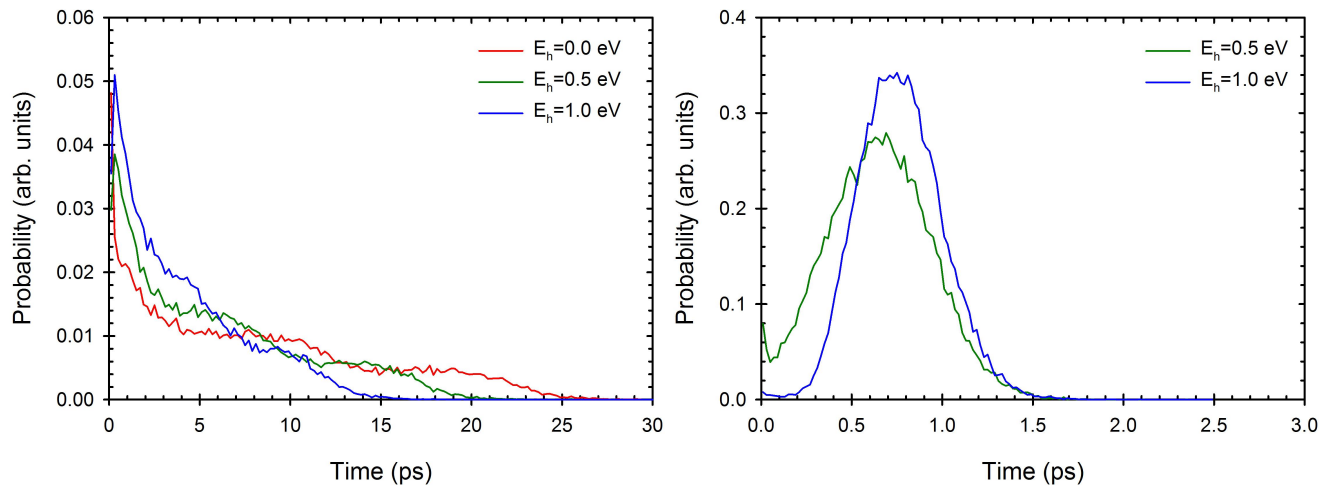

Figure 4. Effective mass approximation model. Time distributions of stopped electrons (left) and holes (right) following irradiation of CsI with 2-keV $\gamma$-rays for three different initial kinetic energies of the holes $\left(\mathrm{E}_{\mathrm{h}}\right)$.

In the second model, the parameterization displayed in Figure 1 and described in section 3.1 was used as input to the KMC simulations. As for the effective mass approximation model, recombination occurs within a few tenths of a picosecond. Figures 5, 6, and 7 show the distance distributions of recombined particles, and the distance distributions and time distributions of stopped particles, respectively. Also shown in these figures, as a reference, are the distributions obtained in our previous publication, ${ }^{22}$ i.e. for an effective electron mass of $1 \mathrm{~m}_{0}$ plus polaron effects over the entire particle energy range and for instantaneous self-trapping of the holes on the lattice.

The distance distributions of recombined particles are quantitatively similar to those obtained with the effective mass approximation model. This result indicates that the distances traveled by particles before recombination are model 
independent and are controlled by the initial densities of electron-hole pairs in different regions of the track, as calculated by NWEGRIM. The distances traveled by electrons before reaching thermal energy are much shorter than those obtained with the effective mass approximation model. The distance distributions peak between 50 and $150 \mathrm{~nm}$ and only extend to 400 to $500 \mathrm{~nm}$. In addition, they are slightly longer than those calculated previously for an effective electron mass of 1 $m_{0}$ plus polaron effects. This is due to the lower effective mass at low energies and the fact that a large proportion of the electrons have low initial energies. In contrast, the holes thermalize over longer distances with the group velocity model, with distance distributions that show a maximum at approximately $20 \mathrm{~nm}$. Nonetheless, there remains a large difference between the extent of hole diffusion and electron diffusion, as expected. For both electrons and holes, the effect of $E_{h}$ on the distance distributions is small. In this model, self-trapping of the holes is assumed to occur once the holes have reached thermal energy; however, if holes can self-trap before reaching thermal energy, their distance and time distributions will likely be reduced as a result. Therefore, the results presented here should be seen as the maximum theoretical thermalization distances and times.
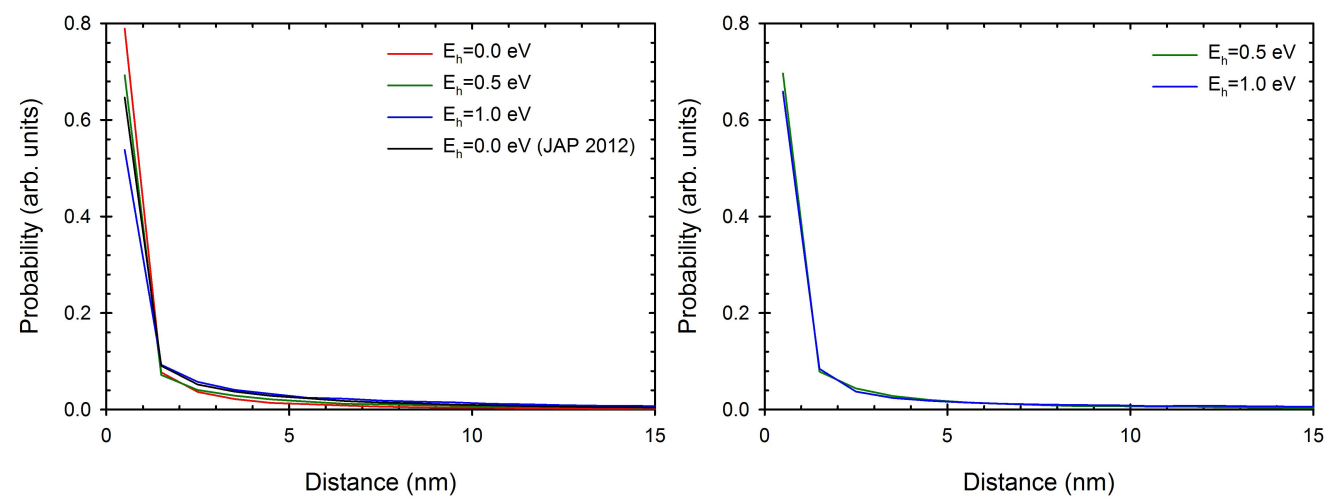

Figure 5. Group velocity model. Distance distributions of recombined electrons (left) and holes (right) following irradiation of CsI with 2-keV $\gamma$-rays for three different initial kinetic energies of the holes $\left(\mathrm{E}_{\mathrm{h}}\right)$. Also shown are the distance distributions obtained in previous work using an electron band mass of $1 \mathrm{~m}_{0}$ with polaron effects.
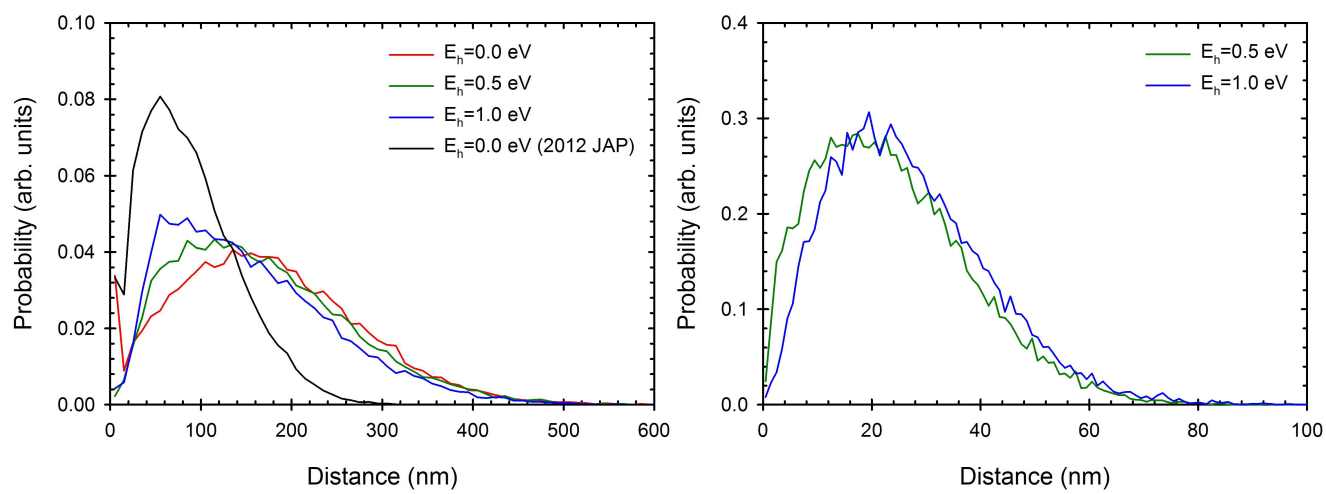

Figure 6. Group velocity model. Distance distributions of stopped electrons (left) and holes (right) following irradiation of CsI with 2-keV $\gamma$-rays for three different initial kinetic energies of the holes $\left(E_{h}\right)$. Also shown are the distance distributions obtained in previous work using an electron band mass of $1 m_{0}$ with polaron effects.

The results obtained with both models are qualitatively similar. Indeed, both models show a significant extent of charge separation between electrons and holes in CsI and a timescale for recombination that is fast compared to the length of the thermalization period. However, the two models exhibit significant quantitative differences. Specifically, the effective mass approximation model predicts much longer electron thermalization distances and times. Because the effective mass approximation is known to only be valid near the conduction band minimum and valence band maximum, the group velocity model is expected to be superior to the effective mass approximation model. Nonetheless, to provide further evidence to justify the use of the group velocity model in future work, a comparison is made with experimental data. 

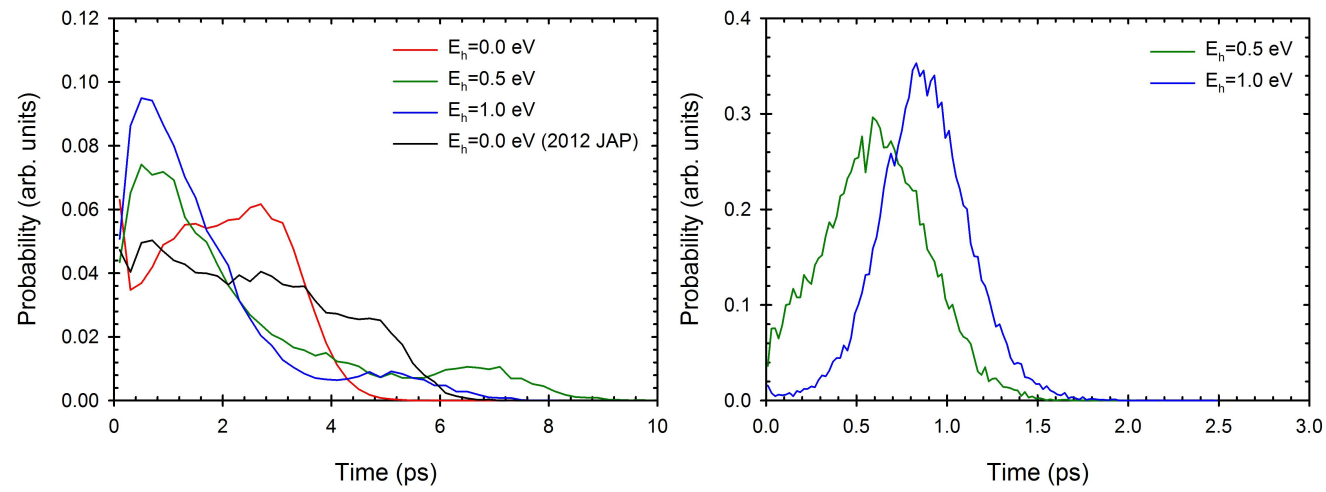

Figure 7. Group velocity model. Time distributions of stopped electrons (left) and holes (right) following irradiation of CsI with 2-keV $\gamma$-rays for three different initial kinetic energies of the holes $\left(\mathrm{E}_{\mathrm{h}}\right)$. Also shown are the distance distributions obtained in previous work using an electron band mass of $1 m_{0}$ with polaron effects.

Experimental data on the length and time scales for electron thermalization in alkali halides are extremely rare. One recent study that offers a possible link between this work and experiment is the picosecond optical absorption study published by Williams and co-workers. ${ }^{23}$ In that work, a pump-probe technique was used whereby CsI was excited by two-photon absorption with excitation energies of 5.9 and $8.86 \mathrm{eV}$. Given a room-temperature band gap of CsI of approximately 5.8-5.9 eV, this results in the formation of free electrons with kinetic energies of approximately 0.1 and $3.0 \mathrm{eV}$ (assuming all the excess kinetic energy is assigned to the electrons). Time-resolved picosecond spectroscopy of the optical absorption induced by the two-photon absorption revealed a delay of $4 \mathrm{ps}$ in the onset of self-trapped exciton formation at $3.0 \mathrm{eV}$ relative to $0.1 \mathrm{eV}$. Therefore, two simulations were performed for each model with electrons with initial kinetic energies of $0.1 \mathrm{eV}$ or $3.0 \mathrm{eV}$ and the delay in thermalization time between the two initial energies was compared with the value of 4 ps obtained experimentally. Because the experimental results are for ultraviolet excitation, an initial random distribution of electron-hole pairs was used together with periodic boundaries conditions. A density of $10^{18}$ electron-hole pairs per $\mathrm{cm}^{3}$ was used as recommended in the work of Williams and co-workers. ${ }^{23}$ Ten simulations with 200 electron-hole pairs each were run in each case and electron-hole pair recombination was turned off to maximize statistics and concentrate on the intrinsic thermalization times. The thermalization time distributions thus obtained are shown in Figure 8.

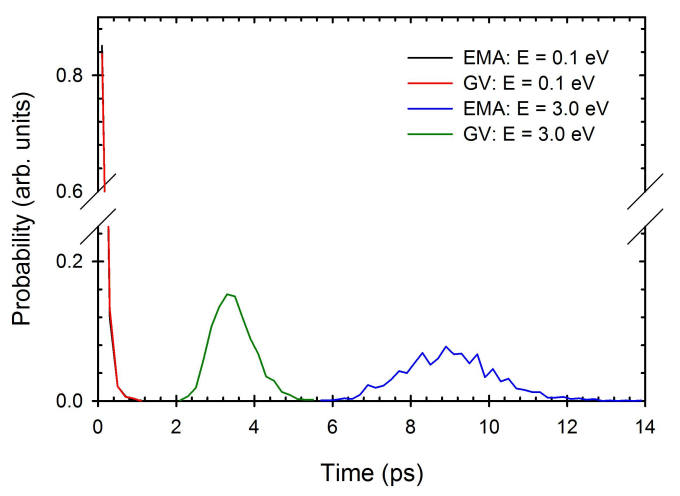

Figure 8. Thermalization time distributions of electrons with kinetic energies of 0.1 or $3.0 \mathrm{~V}$ as obtained with the effective mass approximation (EMA) and group velocity $(\mathrm{GV})$ models.

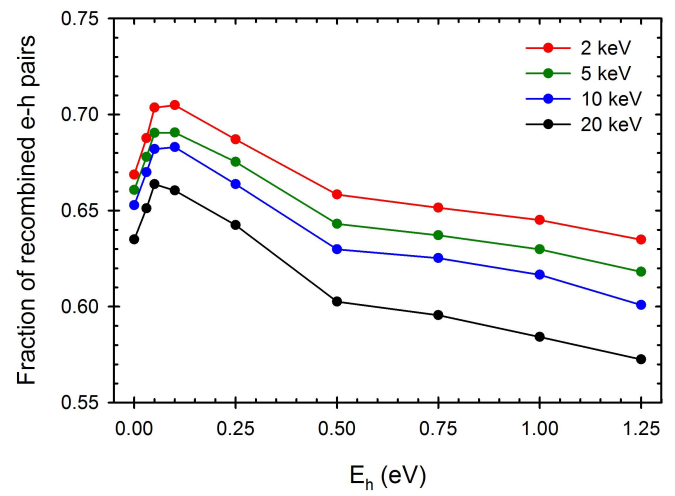

Figure 9. Fraction of recombined electron-hole pairs as a function of initial kinetic energy of the holes $\left(E_{h}\right)$ for incident $\gamma$ ray energies ranging from $2 \mathrm{keV}$ to $20 \mathrm{keV}$.

At $0.1 \mathrm{eV}$, both models predict the large majorities of the electrons to reach thermal energy within $0.1 \mathrm{ps}$. At $3.0 \mathrm{eV}$, the time distribution peaks at approximately $3.4 \mathrm{ps}$ for the group velocity model whereas a broader distribution with a maximum at approximately 9 ps is obtained with the effective mass approximation model. These results indicate that the group velocity model yields good agreement with the time scale obtained experimentally whereas the effective mass approximation model overestimates the electron thermalization time. Therefore, the remainder of this work will focus on the group velocity model. 


\subsection{Group velocity model: effect of incident $\gamma$-ray energy}

The effect of the incident $\gamma$-ray energy on the spatial and temporal extent of thermalization were investigated using the group velocity model and electron-hole pair distributions calculated using NWEGRIM for 2 (400), 5 (200), 10 (100), and 20 (5) $\mathrm{keV} \gamma$-rays, with the number of simulations carried out for each incident energy shown in parentheses. Figure 9 shows the fraction of recombined electron-hole pairs as a function of initial kinetic energy of the holes for the four $\gamma$-ray incident energies. As the incident energy increases, the proportion of regions with a high-density of electron-hole pairs diminishes, which leads to a smaller extent of electron-hole pair recombination regardless of the initial kinetic energy of the holes. For a given incident energy, the fraction of recombined electron-hole pairs initially increases with increasing $E_{h}$ but subsequently decreases as $E_{h}$ increases further. A low initial kinetic energy allows the holes to diffuse over short distances and thus increase their probability of encountering an electron, leading to an increase in the extent of recombination. However, because the sum of the electron and hole kinetic energies is compared to their electrostatic interaction energy to determine whether a recombination event has taken place (section 2.1), high initial kinetic energies of the holes reduce the probability of electron-hole pair recombination. In addition, the probability of electron-hole pair recombination is reduced by the greater dilution of the electron-hole pair spatial distributions caused by assigning holes high initial kinetic energies. Therefore, both factors lead to a decrease in the fraction of recombined electron-hole pairs with increasing initial kinetic energy of the holes.
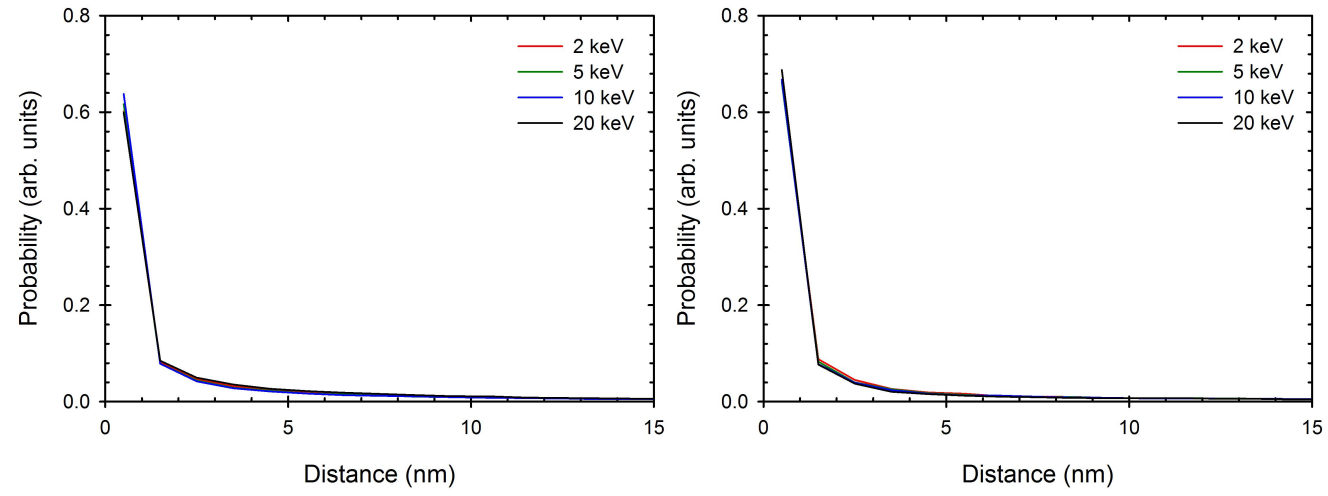

Figure 10. Group velocity model. Distance distributions of recombined electrons (left) and holes (right) for an initial kinetic energy of the holes of $0.75 \mathrm{eV}$ and following irradiation of CsI with $\gamma$-rays of energies ranging from $2 \mathrm{keV}$ to $20 \mathrm{keV}$.

For a given initial kinetic energy of the holes $(0.75 \mathrm{eV}$ for example), the distance distributions of recombined electrons and holes is not affected by the incident $\gamma$-ray energy (Figure 10) because recombination primarily takes place in highdensity regions and only the proportion of these regions changes with incident energy. Similarly, the distance distributions and time distributions of stopped electrons and holes are also little affected by the incident $\gamma$-ray energy (Figures 11 and 12).
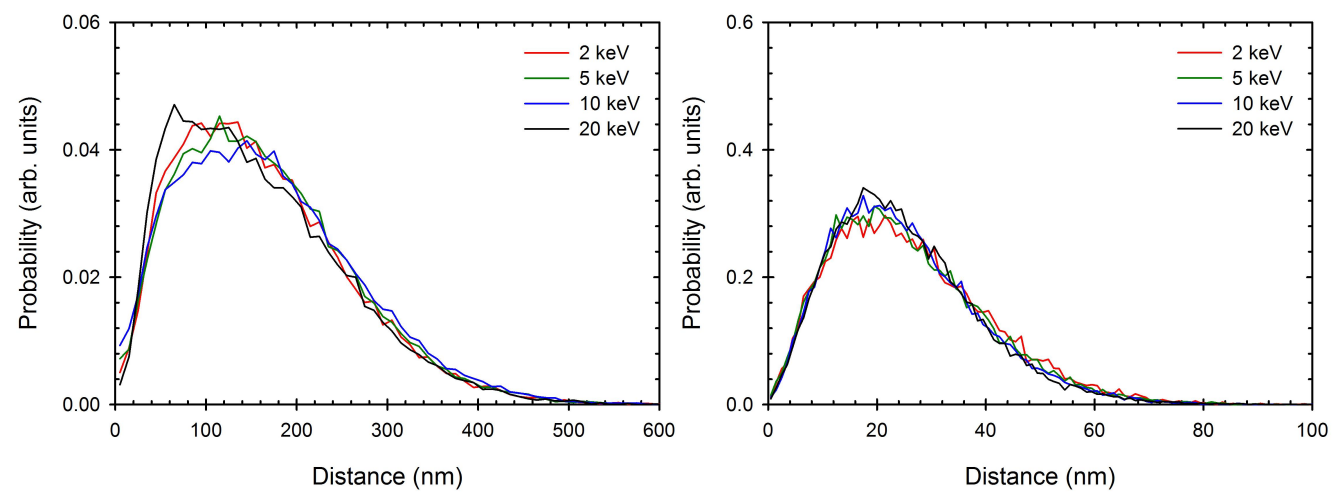

Figure 11. Group velocity model. Distance distributions of stopped electrons (left) and holes (right) for an initial kinetic energy of the holes of $0.75 \mathrm{eV}$ and following irradiation of CsI with $\gamma$-rays of energies ranging from $2 \mathrm{keV}$ to $20 \mathrm{keV}$. 

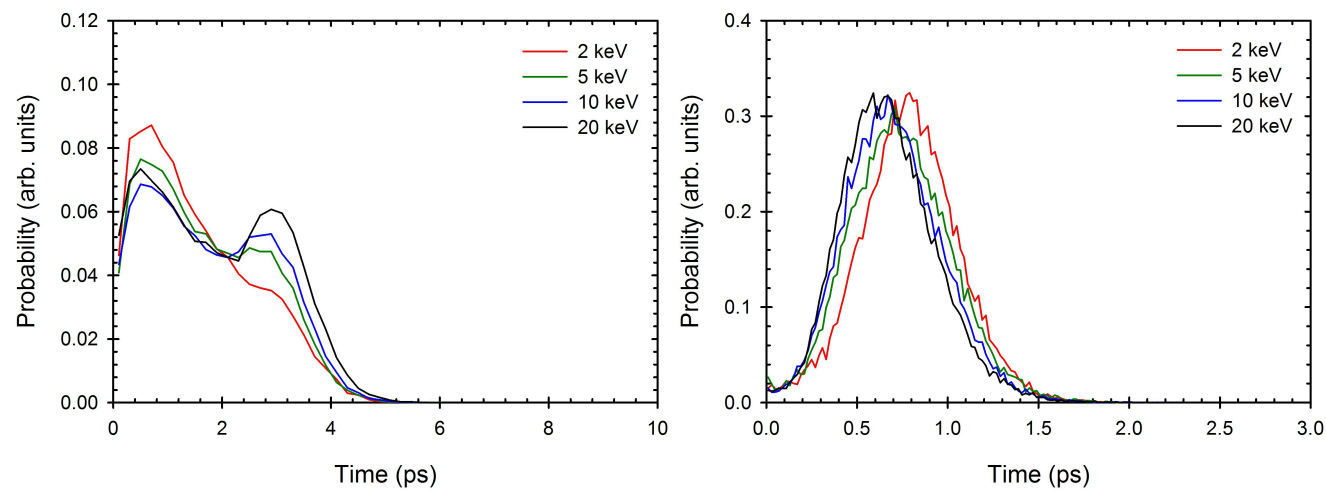

Figure 12. Group velocity model. Time distributions of stopped electrons (left) and holes (right) for an initial kinetic energy of the holes of $0.75 \mathrm{eV}$ and following irradiation of CsI with $\gamma$-rays of energies ranging from $2 \mathrm{keV}$ to $20 \mathrm{keV}$.

\section{DISCUSSION}

The KMC method used to treat thermalization here is semi-classical: the simulation is accomplished by evolving the classical state of a collection of particles (i.e. the positions and momenta of the particles) forward in time with an effective time evolution operator based on quantum mechanical theories. The use of a classical description has many advantages compared to fully quantum approaches based on wave functions or Green's functions. These include greatly reduced computational and storage requirements that enable simulations that are large in space and time. Additionally, the simulation results can be straightforwardly interpreted in familiar, classical terms. However, to realize such a semiclassical scheme, a correspondence must be made between the microscopic, quantum processes and the points of the classical phase space of a single particle so that the microscopic processes can be applied to the classically described particles in the simulation. There is inherent ambiguity here that arises because the classical description of a particle is not compatible with a quantum (wave-mechanical) theory (e.g. interpreting the phase space point corresponding to velocity $\vec{v}$ and position $\vec{r}$ as a simultaneous eigenstate of velocity and position operators violates the uncertainty principle).

Here we focus on the free evolution of particles of a fixed energy $E$ in a crystalline material. A quasi-particle theory of free-particle dynamics in the host material can be obtained from band structure calculations. The band structure $E_{n}(\vec{k})$ is sufficient to predict the time evolution of a given quasi-particle excitation (i.e. a wavefunction describing the state at some initial time expanded in the basis of Bloch waves) via the Schrödinger equation. Excitations consisting of a single Bloch state are not useful for investigating inhomogeneous situations since such excitations extend throughout the crystal and have a time independent density. Hence localized excitations must be constructed as superpositions of the Bloch states. Ehrenfest's theorem implies that a Gaussian wave packet composed of states from the same band $n$ centered at a given crystal momentum $\vec{k}$ will evolve into another Gaussian wave packet, the center of which has moved as a particle whose velocity is given by

$$
\vec{v}_{n}=\frac{d}{d \vec{k}} E_{n}(\vec{k}) .
$$

Therefore, if an ensemble of systems were prepared with the same wave packet (centered at the origin at time 0), the average of position measurements performed a short time $\tau$ later would be

$$
\tau \frac{d}{d \vec{k}} E_{n}(\vec{k}) \text {. }
$$

Hence, Equation 29 has been extensively employed in transport models to treat the free propagation of quasi-particles. The EMA model results from assuming such Bloch dynamics in a band structure consisting of one parabolic band with energy 


$$
E=\frac{k^{2}}{2 m_{b}}
$$

Above, we introduced the GV model (Equation 8) as an extension to the EMA model for the free propagation of quasiparticles in a material that includes more detailed band structure effects but does not require an explicit description of the (quasi)particle's wavefunction. The GV model is simply an average of the speeds given by Equation 29 over all states with the same energy. It contains information about deviations of the conduction band eigenvalues from parabolic dispersion as well as from other bands (which are neglected entirely in the EMA model). As can be seen from Figure 1, the EMA and GV models agree well for electrons within about $0.5 \mathrm{eV}$ of the conduction band minimum. The reason for this agreement is that, in this energy range, the only states available are the conduction band states derived from the Cs $6 s$ states, and their energies are approximated well by Equation 31. Above this energy, other bands become accessible to electrons. The bottoms of the new bands (where the gradient vanishes) contribute first as energy is increased, leading to a sharp drop in the average group velocity compared to the EMA model. The flatter bands of the computed band structure compared to the parabolic fit at the conduction band minimum lead to significantly smaller average group velocities for electrons in CsI at all higher energies, too. This is a general feature that can be expected in a wide range of materials.

Insight into the differences between the EMA and GV models can be found by noting that, when projected into the first Brillouin zone, the free-particle band structure (Equation 31 of the EMA model) has sharp kinks at the Brillouin zone boundary where the bands are folded back. In contrast, the actual band structure of CsI must have the periodicity of the reciprocal lattice. This means that the bands must "bend over" to avoid slope discontinuities at the Brillouin zone boundaries. It follows that any quasi-particle band structure of a crystal will have states (at high-symmetry points) where the gradient of the band structure (and hence the group velocity) vanishes for every band; the EMA model has only one zero-velocity point at $\vec{k}=\overrightarrow{0}$. These states have arbitrarily high energy, but Bloch dynamics predict that they do not move as time evolves.

However, if instead of averaging the vector positions of the time-evolved ensembles first and then computing the distance traveled as the magnitude of the difference between the average initial position and average final position to find the speed of quasi-particle propagation, one were to compute the distance traveled for each system in the ensemble first and then take the ensemble average, different (larger) results would be obtained, i.e.

$$
|\langle\bar{r}(\tau)\rangle| \leq\langle|\bar{r}(\tau)|\rangle \text {. }
$$

This is because the vector positions can interfere destructively while the (non-negative) distances always add constructively in the ensemble average. Bloch dynamics capture the drift of the wave-packet (the motion associated with the group velocity) but neglect the diffusive effects of the spreading of the wave-packet. The latter become more important as the extent of the original wave-packet is decreased (i.e. the particle is more localized) and also when the group velocity has a small magnitude because the band structure is locally shallow. Hence, if one wants to know how far a particle has traveled in a given time, Bloch dynamics give a lower bound for wave packets moving in a prescribed band, becoming exact as the size of the initial wave packets grows. When the EMA model is employed for free-particle transport, there are two kinds of errors made: diffusive motion is ignored (the particle moves with its group velocity only) and the group velocity is overestimated. These two errors typically have opposite signs, so they may partially cancel. The GV model is clearly an improvement over the EMA model for the Bloch dynamics of high energy quasi-particles.

There are other plausible ways to estimate the velocity with which a particle moves through a material than the ones presented above. We have investigated other such prescriptions. An interesting one is the expected value of the velocity, i.e. the magnitude of the physical momentum divided by the particle mass. This prescription gives much larger values for the velocity than the gradient of the band structure presented above and has the obvious defect that it does not vanish for any particle in the material. In fact, it is quite large for bound electrons, which should not contribute to the transport. However, it does approach the free particle result much more quickly than the band structure gradient at high electron energy and becomes comparable to the effective mass approximation for energies near the cut-off energy below which an electron cannot excite additional electronic excitations. 


\section{CONCLUSIONS}

KMC calculations were carried out to simulate the thermalization of hot electrons and holes in CsI following irradiation with $\gamma$-rays of energies ranging between $2 \mathrm{keV}$ and $20 \mathrm{keV}$. The calculations employed a semi-classical particle-phonon interaction model based on Fröhlich scattering for interaction with longitudinal optical phonons and the formulation of Sparks et $a l^{36}$ for interactions with acoustic phonons. In addition, two models of quasi-particle dispersion were considered: the effective mass approximation model and a group velocity model used to account for the presence of multiple energy bands for particle energies greater than a fraction of one electronvolt.

The two models offer qualitatively similar results. For example, electron-hole pair recombination is predicted by both models to be fast (a few tens of a picosecond) and to take place over a limited spatial range (a few nanometers). Importantly, both models predict thermalization to lead to a considerable extent of charge separation in CsI. However, quantitative predictions of the spatial and temporal scales required for particles to reach thermal energy differ significantly for the two models. Comparison of the results obtained with the two models for the thermalization of electrons with initial kinetic energies of $0.1 \mathrm{eV}$ and $3.0 \mathrm{eV}$ with the picosecond optical absorption data of Ucer et al. ${ }^{23}$ indicated that the group velocity model yielded more accurate predictions.

Finally, as discussed in the Introduction section, the time during which electrons and holes persist as free particles directly affects the extent of third-order nonlinear quenching, which, in turn, plays a critical role in determining the magnitude of the deviation from proportional response of a given inorganic scintillator. Both models employed in this work indicate that hole thermalization is faster than electron thermalization, thus suggesting that the time scale for hole thermalization might constitute a controlling factor for the rate and magnitude of third-order nonlinear quenching. Future studies will extend the predictions made in this work to other common and emerging scintillators (e.g. NaI, YAP, and $\mathrm{SrI}_{2}$ ).

\section{ACKNOWLEDGMENTS}

This work was supported by the National Nuclear Security Administration, Office of Nuclear Nonproliferation Research and Engineering (NA-22), of the U.S. Department of Energy.

\section{REFERENCES}

[1] Jaffe, J.E., Jordan, D.V. and Peurrung, A.J., "Energy nonlinearity in radiation detection materials: Causes and consequences," Nucl. Instr. and Meth. A 570 72-83 (2007).

[2] Collinson, A.J.L. and Hill, R., "The fluorescent response of $\mathrm{NaI}(\mathrm{Tl})$ and $\mathrm{CsI}(\mathrm{Tl})$ to $\mathrm{X}$ rays and $\gamma$ rays," Proc. Phys. Soc. 81 883-892 (1963).

[3] Choong, W.-S., Hull, G., Moses, W.W., Vetter, K.M., Payne, S.A., Cherepy, N.J. and Valentine, J.D., "Performance of a facility for measuring scintillator non-proportionality," IEEE Trans. Nucl. Sci. 55 (3), 1073-1078 (2008).

[4] Choong, W.-S., Vetter, K.M., Moses, W.W., Hull, G., Payne, S.A., Cherepy, N.J. and Valentine, J.D., "Design of a facility for measuring scintillator non-proportionality," IEEE Trans. Nucl. Sci. 55 (3), 1753-1758 (2008).

[5] Moses, W.W., Payne, S.A., Choong, W.-S., Hull, G. and Reutter, B.W., "Scintillator non-proportionality: Present understanding and future challenges," IEEE Trans. Nucl. Sci. 55 (3), 1049-1053 (2008).

[6] Vasil'ev, A.N., "From luminescence non-linearity to scintillation non-proportionality," IEEE Trans. Nucl. Sci. 55 (3), 1054-1061 (2008).

[7] Bizarri, G., Moses, W.W., Singh, J., Vasil'ev, A.N. and Williams, R.T., "An analytical model of nonproportional scintillator light yield in terms of recombination rates," J. Appl. Phys. 105044507 (2009).

[8] Kerisit, S., Rosso, K.M., Cannon, B.D., Gao, F. and Xie, Y., "Computer simulation of the light yield nonlinearity of inorganic scintillators," J. Appl. Phys. 105114915 (2009).

[9] Payne, S.A., Cherepy, N.J., Hull, G., Valentine, J.D., Moses, W.W. and Choong, W.-S., "Nonproportionality of scintillator detectors: theory and experiment," IEEE Trans. Nucl. Sci. 56 (4), 2506-2512 (2009). 
[10] Setyawan, W., Gaume, R.M., Feigelson, R.S. and Curtarolo, S., "Comparative study of nonproportionality and electronic band structures features in scintillator materials," IEEE. Trans. Nucl. Sci. 56 (5), 2989-2996 (2009).

[11] Li, Q., Grim, J.Q., Williams, R.T., Bizarri, G.A. and Moses, W.W., "A transport-based model of material trends in nonproportionality of scintillators," J. Appl. Phys. 109123716 (2011).

[12] Payne, S.A., Moses, W.W., Sheets, S., Ahle, L., Cherepy, N.J., Sturm, B., Dazeley, S., Bizarri, G. and Choong, W.-S., "Nonproportionality of scintillator dectectors: theory and experiment. II," IEEE Trans. Nucl. Sci. 58 (6), $3392-$ $3402(2011)$.

[13] Singh, J., "Study of nonproportionality in the light yield of inorganic scintillators," J. Appl. Phys. 110024503 (2011).

[14] Williams, R.T., Grim, J.Q., Li, Q., Ucer, K.B. and Moses, W.W., "Excitation density, diffusion-drift, and proportionality in scintillators," Phys. Status Solidi B 248 (2), 426-438 (2011).

[15] Moses, W.W., Bizarri, G.A., Williams, R.T., Payne, S.A., Vasil'ev, A.N., Singh, J., Li, Q., Grim, J.Q. and Choong, W.-S., "The origins of scintillator non-proportionality," IEEE Trans. Nucl. Sci. 59 (5), 2038-2044 (2012).

[16] Grim, J.Q., Ucer, K.B., Burger, A., Bhattacharya, P., Tupitsyn, E., Rowe, E., Buliga, V.M., Trefilova, L., Gektin, A., Bizarri, G.A., Moses, W.W. and Williams, R.T., "Nonlinear quenching of densely excited states in wide-gap solids," Phys. Rev. B 87125117 (2013).

[17] Peurrung, A., "Materials science for nuclear detection," Mater. Today 11 (3), 50-54 (2008).

[18] Wang, Z., Xie, Y.-L., Cannon, B.D., Campbell, L.W., Gao, F. and Kerisit, S., "Computer simulation of electron thermalization in CsI and CsI(Tl)," J. Appl. Phys. 110064903 (2011).

[19] Kirkin, R., Mikhailin, V.V. and Vasil'ev, A.N., "Recombination of correlated electron-hole pairs with account of hot capture with emission of optical phonons," IEEE Trans. Nucl. Sci. 59 (5), 2057-2064 (2012).

[20] Kozorezov, A., Wigmore, J.K. and Owens, A., "Picosecond dynamics of hot carriers and phonons and scintillator non-proportionality," J. Appl. Phys. 112053709 (2012).

[21] Li, Q., Grim, J.Q., Ucer, K.B., Burger, A., Bizarri, G.A., Moses, W.W. and Williams, R.T., "Host structure dependence of light yield and proportionality in scintillators in terms of hot and thermalized carrier transport," Phys. Status Solidi RRL 6 (8), 346-348 (2012).

[22] Wang, Z., Xie, Y.-L., Campbell, L.W., Gao, F. and Kerisit, S., "Monte Carlo simulations of electron thermalization in alkali iodide and alkaline-earth fluoride scintillators," J. Appl. Phys. 112014906 (2012).

[23] Ucer, K.B., Bizarri, G., Burger, A., Gektin, A., Trefilova, L. and Williams, R.T., "Electron thermalization and trapping rates in pure and doped alkali and alkaline-earth iodide crystals studied by picosecond optical absorption," Phys. Rev. B 89165112 (2014).

[24] Vasil'ev, A.N. and Gektin, A.V., "Multiscale approach to estimation of scintillation characteristics," IEEE Trans. Nucl. Sci. 61 (1), 235-245 (2014).

[25] Williams, R.T., Grim, J.Q., Li, Q., Ucer, K.B., Bizarri, G.A., Kerisit, S., Gao, F., Bhattacharya, P., Tupitsyn, E., Rowe, E., Buliga, V.M. and Burger, A., "Experimental and computational results on exciton/free-carrier ratio, hot/thermalized carrier diffusion, and linear/nonlinear rate constants affecting scintillator proportionality," Proc. SPIE $885288520 \mathrm{~J}(2013)$.

[26] Kerisit, S., Rosso, K.M. and Cannon, B.D., "Kinetic Monte Carlo model of scintillation mechanisms in CsI and CsI(Tl)," IEEE Trans. Nucl. Sci. 55 (3), 1251-1258 (2008).

[27] Wang, Z., Williams, R.T., Grim, J.Q., Gao, F. and Kerisit, S., "Kinetic Monte Carlo simulations of excitation density dependent scintillation in CsI and CsI(Tl)," Phys. Status Solidi B 250 (8), 1532-1540 (2013).

[28] Gao, F., Campbell, L.W., Xie, Y., Devanathan, R., Peurrung, A.J. and Weber, W.J., "Electron-hole pairs created by photons and intrinsic properties in detector materials," IEEE Trans. Nucl. Sci. 55 (3), 1079-1085 (2008).

[29] Gao, F., Xie, Y., Kerisit, S., Campbell, L.W. and Weber, W.J., "Yield, variance and spatial distribution of electron-hole pairs in CsI," Nucl. Instr. and Meth. Phys. Res. A 652 564-567 (2011).

[30] Gao, F., Xie, Y.-L., Wang, Z.G., Kerisit, S., Wu, D.X., Campbell, L.W., Van Ginhoven, R.M. and Prange, M., "Monte Carlo simulation of gamma-ray response of $\mathrm{BaF}_{2}$ and $\mathrm{CaF}_{2}$," J. Appl. Phys. 114173512 (2013).

[31] Shimizu, R. and Ze-Jun, D., "Monte Carlo modeling of electron-solid interactions," Rep. Prog. Phys. 55 487531 (1992).

[32] Van Ginhoven, R.M., Jaffe, J.E., Kerisit, S. and Rosso, K.M., "Trapping of holes and excitons in scintillators: CsI and $\mathrm{LaX}_{3}(\mathrm{X}=\mathrm{Cl}, \mathrm{Br}), "$ IEEE Trans. Nucl. Sci. 57 2303-2308 (2010).

[33] Boutboul, T., Akkerman, A., Breskin, A. and Chechik, R., "Escape length of ultraviolet induced photoelectrons in alkali iodide and CsBr evaporated films: Measurements and modeling," J. Appl. Phys. 84 2890-2896 (1998).

[34] Reinitz, K., "Elastic constants of CsBr, CsI, RbBr, and RbI," Phys. Rev. 123 1615-1619 (1961). 
[35] Williams, R.T. and Song, K.S., "The self-trapped exciton," J. Phys. Chem. Solids 51 679-716 (1990).

[36] Sparks, M., Mills, D.L., Warren, R., Holstein, T., Maradudin, A.A., Sham, L.J., Loh, E., Jr. and King, D.F., "Theory of electron-avalanche breakdown in solids," Phys. Rev. B 24 (6), 3519-3536 (1981).

[37] Ashley, J.C., Ritchie, R.H. and Crawford, O.H., "Energy loss and scattering of subexcitation electrons in $\mathrm{SiO}_{2}$," 10th Werner Brandt Conference, Alicante, Spain, 329 (1988)

[38] Fischetti, M.V., "Monte Carlo solution to the problem of high-field electron heating in $\mathrm{SiO}_{2}$," Phys. Rev. Lett. 53 1755-1758 (1984).

[39] Ankudinov, A.L., Ravel, B., Rehr, J.J. and Conradson, S.D., "Real-space multiple-scattering calculation and interpretation of x-ray-absorption near-edge structure," Phys. Rev. B 58 (12), 7565-7576 (1998).

[40] Bradford, J.N. and Woolf, S., "Electron-acoustic phonon scattering in $\mathrm{SiO}_{2}$ determined from a pseudo-potential for energies of $\mathrm{E}>\mathrm{E}_{\mathrm{BZ}}$," J. Appl. Phys. 70 490-493 (1991).

[41] Fischetti, M.V., DiMaria, D.J., Brorson, S.D., Theis, T.N. and Kirtley, J.R., "Theory of high-field electron transport in silicon dioxide," Phys. Rev. B 31 8124-8142 (1985).

[42] Gonze, X., Amadon, B., Anglade, P.-M., Beuken, J.-M., Bottin, F., Boulanger, P., Bruneval, F., Caliste, D., Caracas, R., Côté, M., Deutsch, T., Genovese, L., Ghosez, P., Giantomassi, M., Goedecker, S., Hamann, D.R., Hermet, P., Jollet, F., Jomard, G., Leroux, S., Mancini, M., Mazevet, S., Oliveira, M.J.T., Onida, G., Pouillon, Y., Rangel, T., Rignanese, G.-M., Sangalli, D., Shaltaf, R., Torrent, M., Verstraete, M.J., Zerah, G. and Zwanziger, J.W., "ABINIT: First-principles approach to materials and nanosystem properties," Computer Phys. Comm. 180 2582-2615 (2009).

[43] Goedecker, S., Teter, M. and Hutter, J., "Separable dual-space Gaussian pseudopotentials," Phys. Rev. B 54 (3), 1703-1710 (1996).

[44] Perdew, J.P., Burke, K. and Ernzerhof, M., "Generalized gradient approximation made simple," Phys. Rev. Lett. 77 (18), 3865-3868 (1996).

[45] Perdew, J.P., Burke, K. and Ernzerhof, M., "Generalized gradient approximation made simple [Phys. Rev. Lett. 77, 3865 (1996)]," Phys. Rev. Lett. 78 (7), 1396 (1997). 


\section{Science-Driven Candidate Search for New Scintillator Materials}

\section{Sebastien Kerisit, Yulong Xie, Luke W. Campbell, Micah P. Prange, Dangxin Wu, and Fei Gao (PI)}

\section{Pacific Northwest National Laboratory}

This project is funded by the National Nuclear Security Administration, Office of Defense Nuclear Nonproliferation, Office of Nonproliferation and Validation Research \& Development (DNN R\&D/NA-22), U.S. Department of Energy.

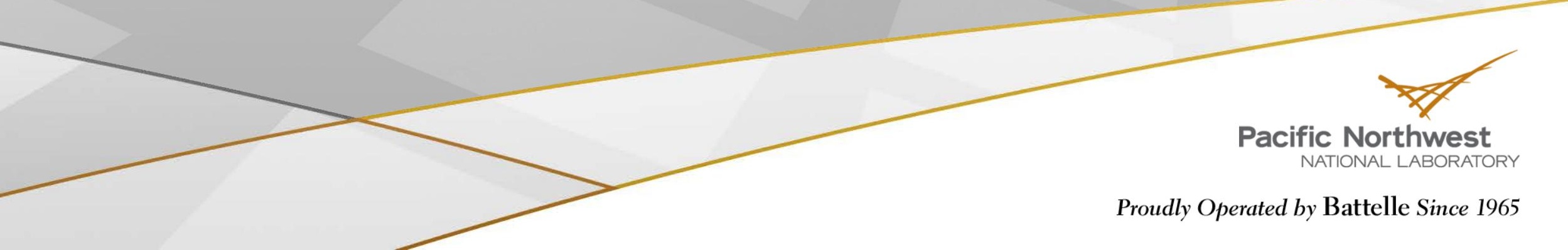




\section{Motivation \& Objective}

\section{Motivation}

- Why some scintillator materials offer better performance than others? (e.g. nonproportionality)

- What are the fundamental performance limits of a given scintillator material?

- Fundamental physical processes that control scintillator performance are not well understood.

- Need for developing comprehensive scintillator physics models/computer codes.

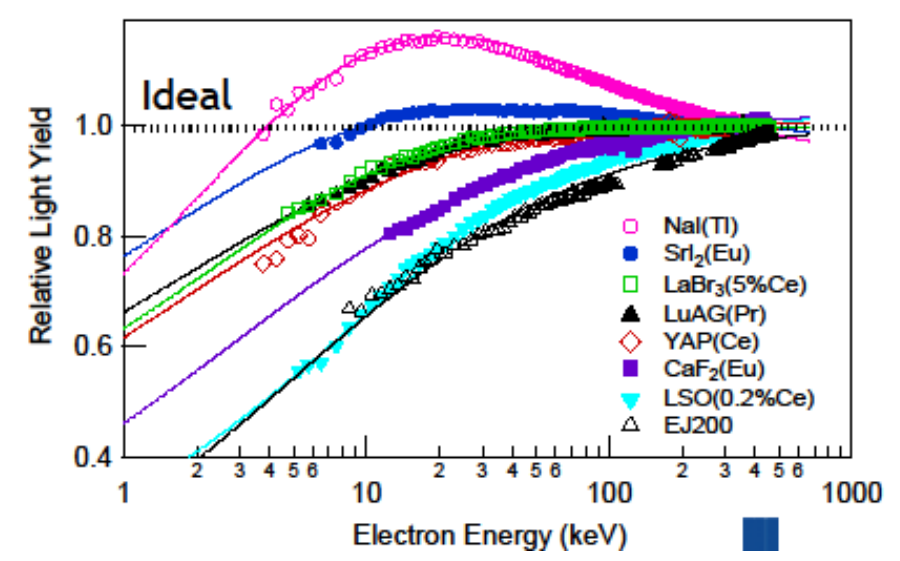

S. Payne - SPIE meeting 2011

\section{Objective}

\section{Develop predictive models to understand radiation response}

Thereby providing a theoretical basis to:

- Improve the performance of current scintillators

- Accelerate the candidate search for new scintillator materials 


\section{Overview of Approach}

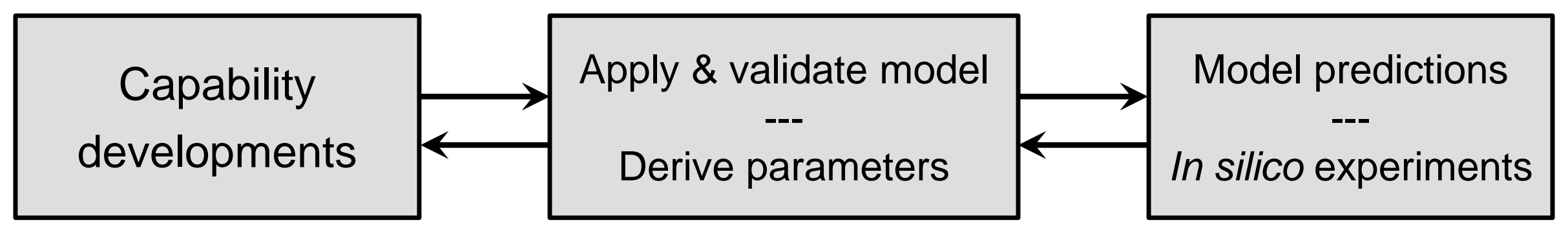

\section{Capability developments}

- Electronic ReSPonse (ERSP)

Low-energy cross sections and plasmon decay spectra

- NorthWest Electron \& Gamma-Ray Interaction with Matter (NWEGRIM)

Energy cascade $\rightarrow$ generation of electron-hole pairs

- Deformable Density (DefDen) model

Electron/hole-phonon interactions

- Kinetic Monte Carlo (KMC) code

Carrier thermalization, transport, recombination, and luminescence

\section{Model applications and predictions}

- Wide range of scintillators (alkali/alkaline-earth/lanthanum halides, oxides, elpasolites)

- Light yields, decay kinetics, nonproportionality, pulse shape discrimination

- Establish rules that dictate scintillator performance (self-consistent analysis)

- Material engineering (co-doping, defect manipulation) 

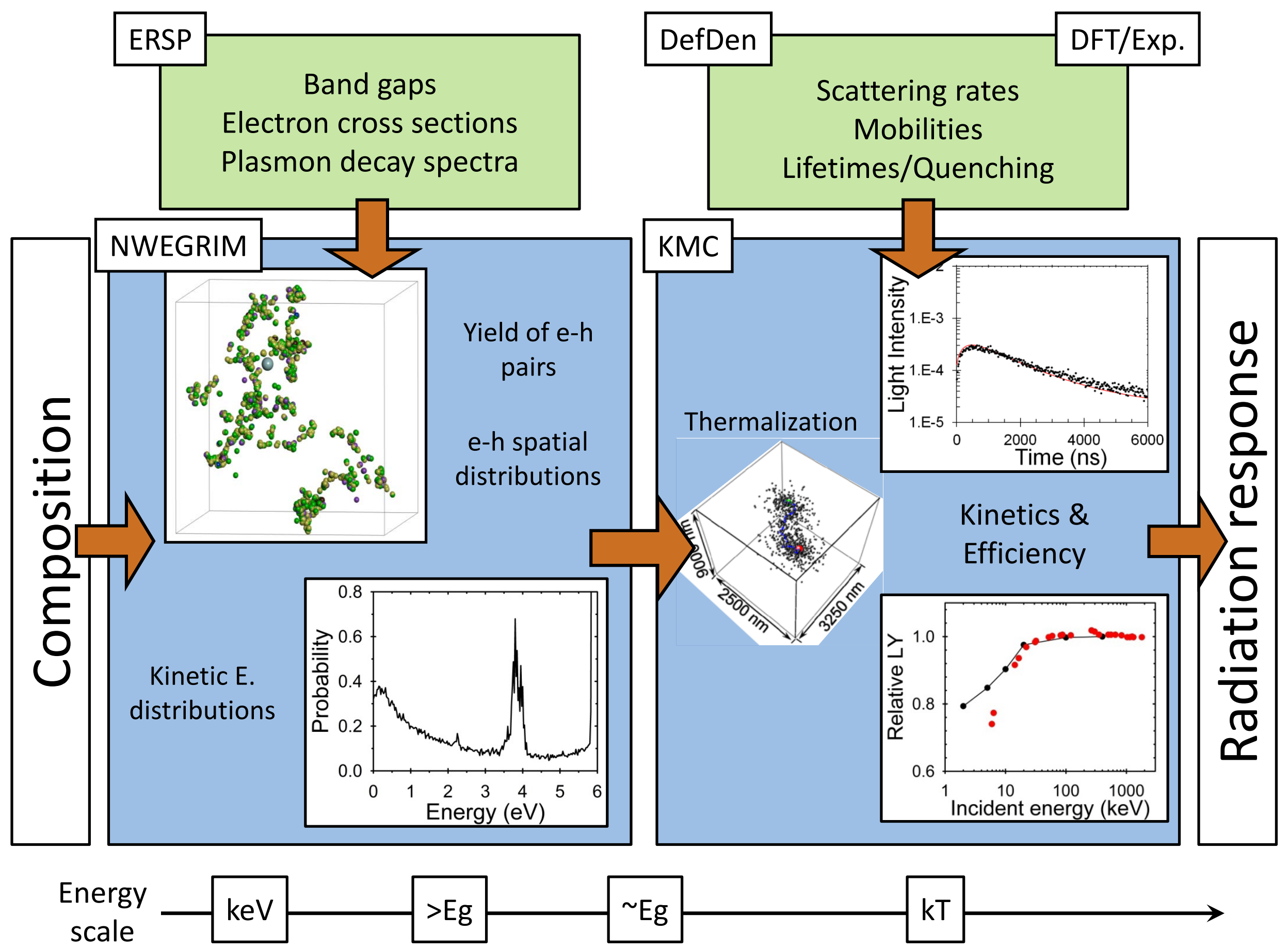


\section{Ab initio calculations of electronic excitations}

$\checkmark$ Methods

L.W. Campbell

- Sum over possible excitations in the material to determine

- Polarizability,

- Rate of creation of excitations.

$\checkmark$ Used to find

- Interaction cross sections, power loss, and mean free path of low-energy electrons

- Fit low-energy cross section of NWEGRIM

- Spectra of particles created by electron

- Provide spectrum of secondary particles from plasmon decay for NWEGRIM

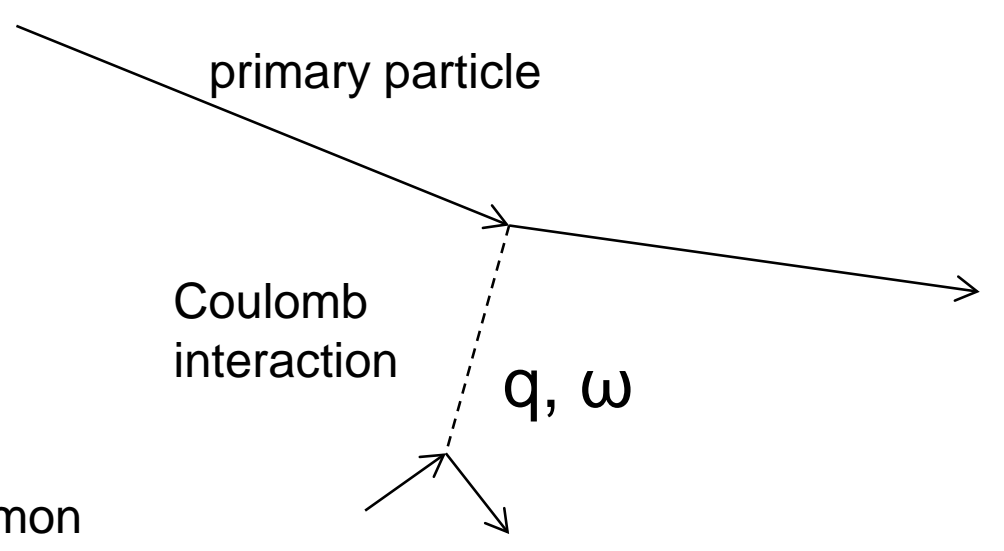

secondary particle

\section{Example: $\mathrm{BaF}_{2}$}

Low-energy behavior is critical to capturing fluctuations near the track end
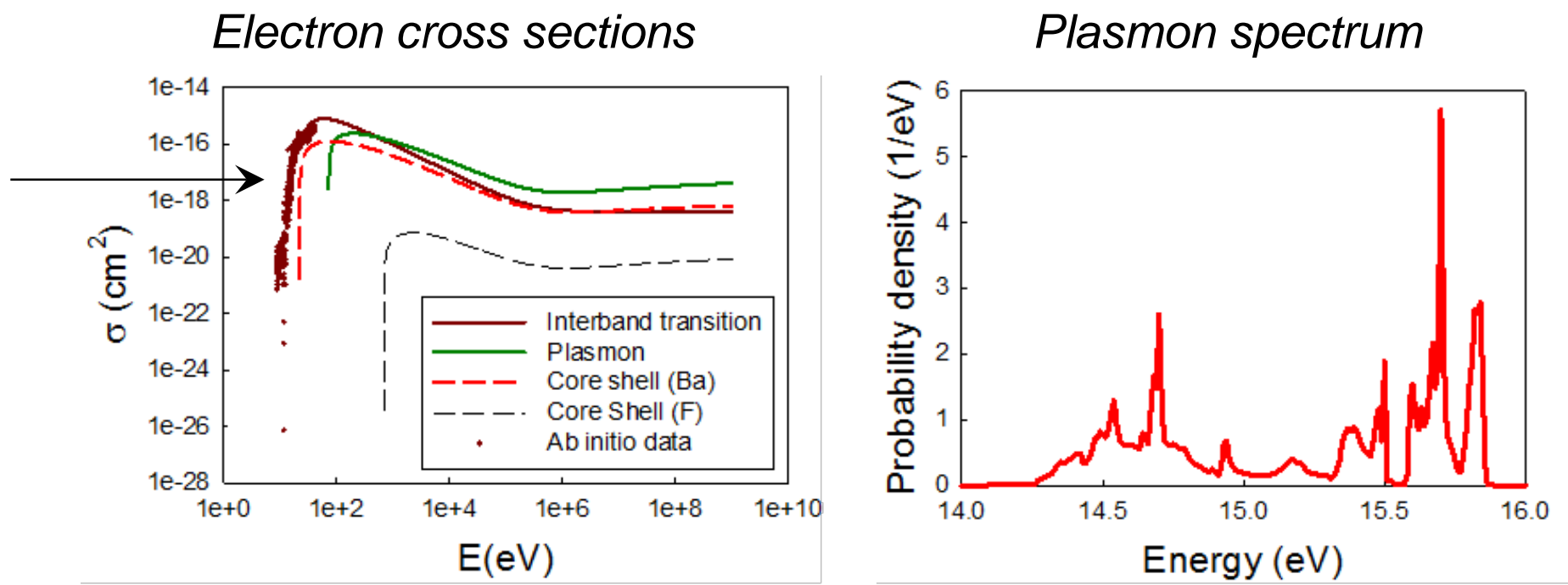


\section{Ab initio calculations of electronic excitations}

\section{L.W. Campbell}

All experimental electron energy loss function peaks reproduced (albeit shifted by $\sim 2-4 \mathrm{eV}$ ) Campbell and Gao J. Lumin. 137, 121 (2013)

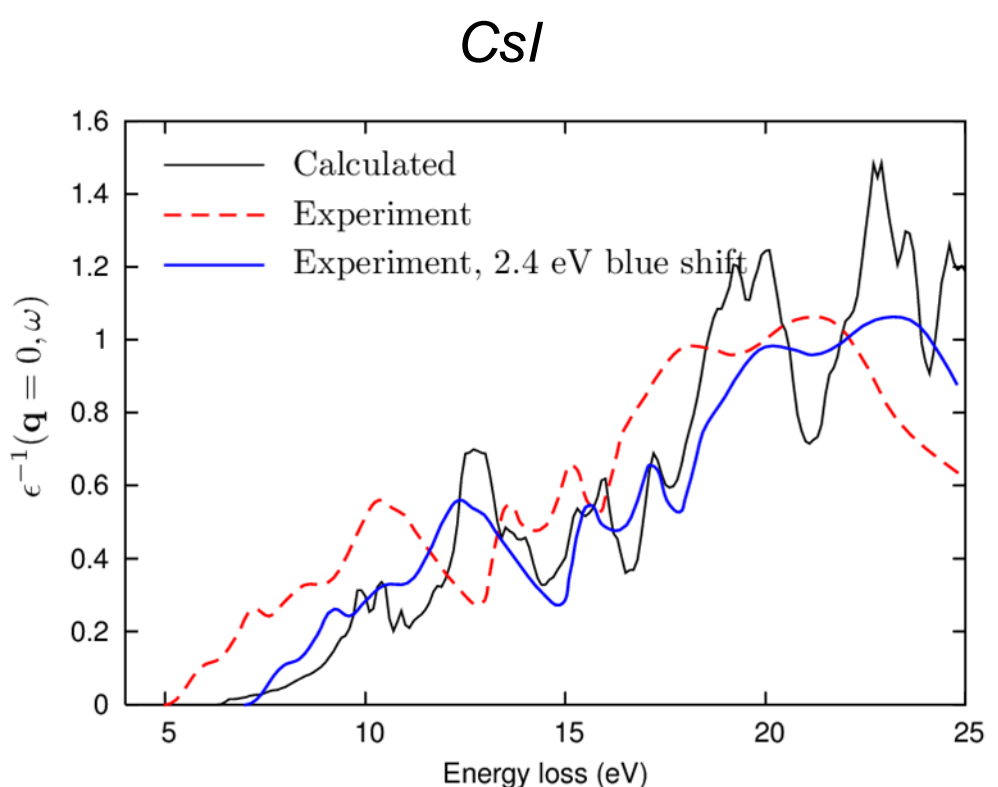

Index of refraction $n_{\infty}$

\begin{tabular}{|c|c|c|}
\hline & Experiment & Theory \\
\hline $\mathrm{Csl}$ & 1.74 & 1.64 \\
\hline $\mathrm{Nal}$ & 1.73 & 1.69 \\
\hline
\end{tabular}

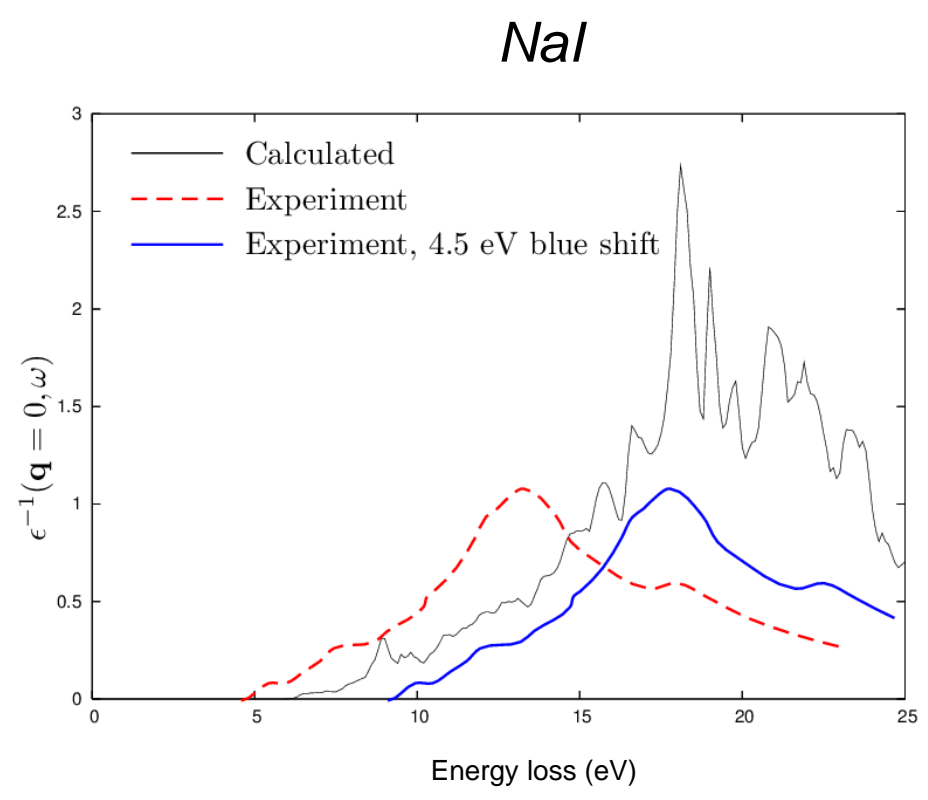

$>$ Optical band gap (eV)

\begin{tabular}{|c|c|c|}
\hline & Experiment & Theory \\
\hline Csl & $6.0-6.4$ & 5.5 \\
\hline Nal & $5.75-5.9$ & 6.07 \\
\hline
\end{tabular}




\section{Electronic losses of heavy particles}

L.W. Campbell

- Significant interest in materials that scintillate under neutron radiation

- Fast neutrons detected by nuclear recoil from collisions with neutrons (esp. protons)

- Slow neutrons detected by absorption interactions

n $\mathrm{n}+{ }^{6} \mathrm{Li} \rightarrow \mathrm{\alpha}+\mathrm{T}$

n $\mathrm{n}+{ }^{10} \mathrm{~B} \rightarrow \mathrm{\alpha}+{ }^{7} \mathrm{Li}$

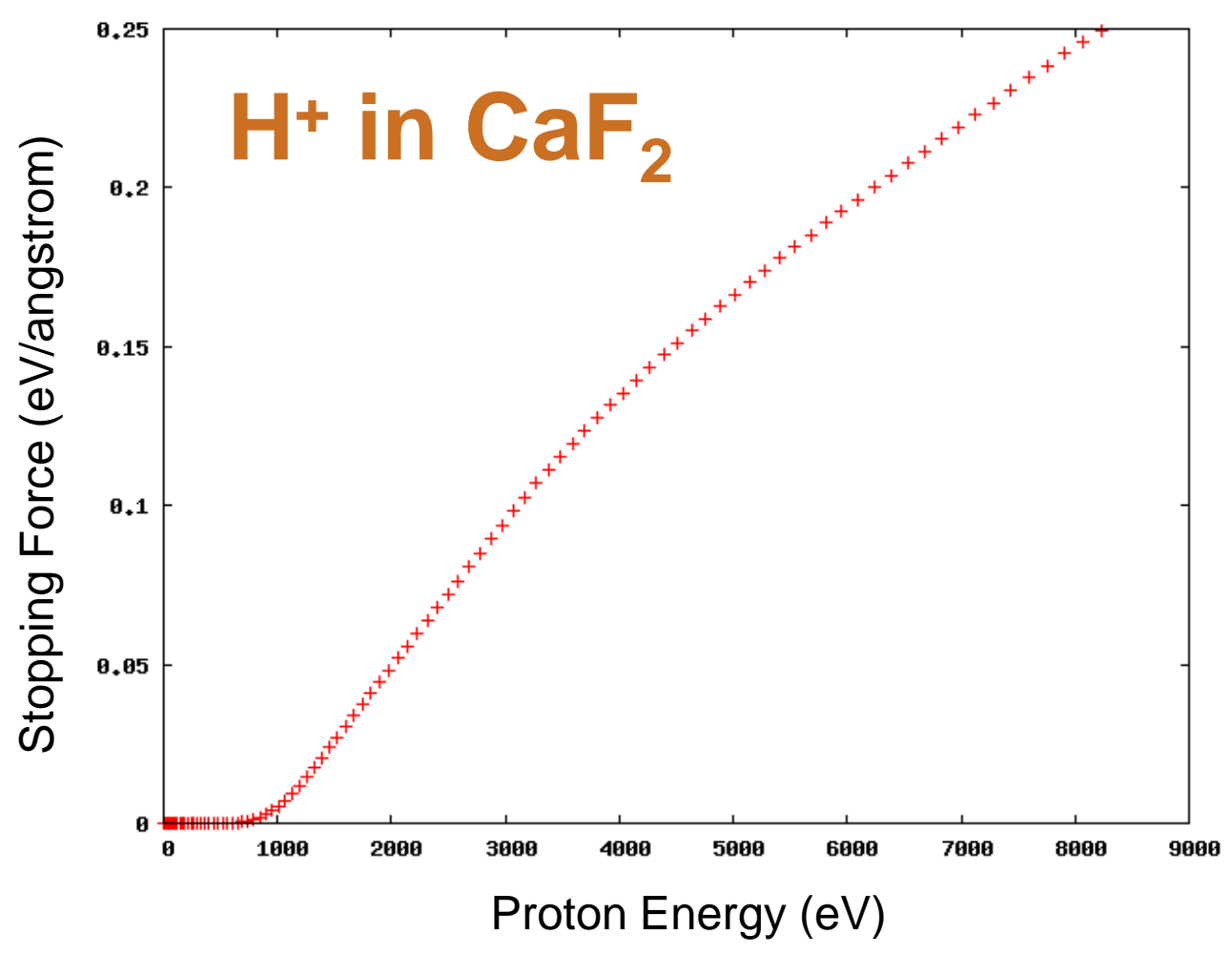

- Need to be able to predict scintillation behavior of energetic nuclei

- Preliminary code completed for heavy ion response in ERSP

- Predict interaction rates and power loss

- First step towards simulating dual detectors (e.g. elpasolites) 


\section{NW Electron \& Gamma-Ray Interactions in Matter (NWEGRIM)}

\section{Features}

- Low cutoff energy $\left(\sim E_{g}\right) \rightarrow$ explicit account of all ionization events

- Average energy per electron-hole pair is predicted not an input parameter

- Multiple elastic scattering theory

- Database of various electron cross sections

- Incorporate ab initio data model predictions

\section{Properties}

- Spatial distribution of e-h pairs - track structures

- W vs. particle E, max. theo. LY, and Fano factor

- Energy loss channels and stopping power

\section{Scintillator materials}

- Alkali and alkaline-earth halides (Nal, $\mathrm{CsI}_{1} \mathrm{BaF}_{2}, \mathrm{CaF}_{2}, \mathrm{Srl}_{2}$ )

Mean energy per e-h pair

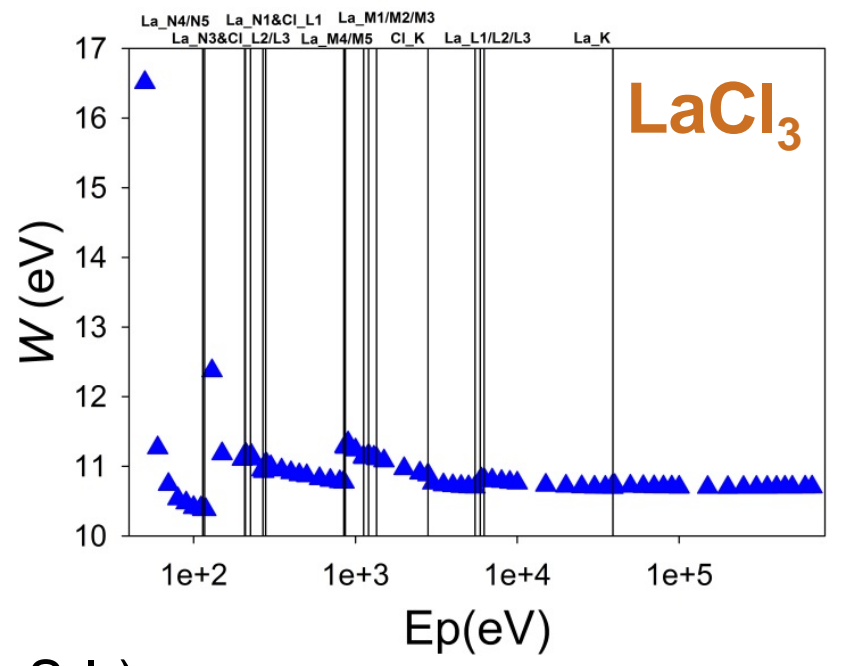

- Lanthanum halide series $\left(\mathrm{LaF}_{3}, \mathrm{LaCl}_{3}, \mathrm{LaBr}_{3}, \mathrm{Lal}_{3}\right)$

- Oxides (YAG, YAP)

- Elpasolites (CLLC, CLLC, CLYB, CLYC)

D. Wu, Y. Xie, F. Gao 


\section{Examples of y-ray ionization tracks}

$10 \mathrm{keV}$ incident $\mathrm{y}$-ray
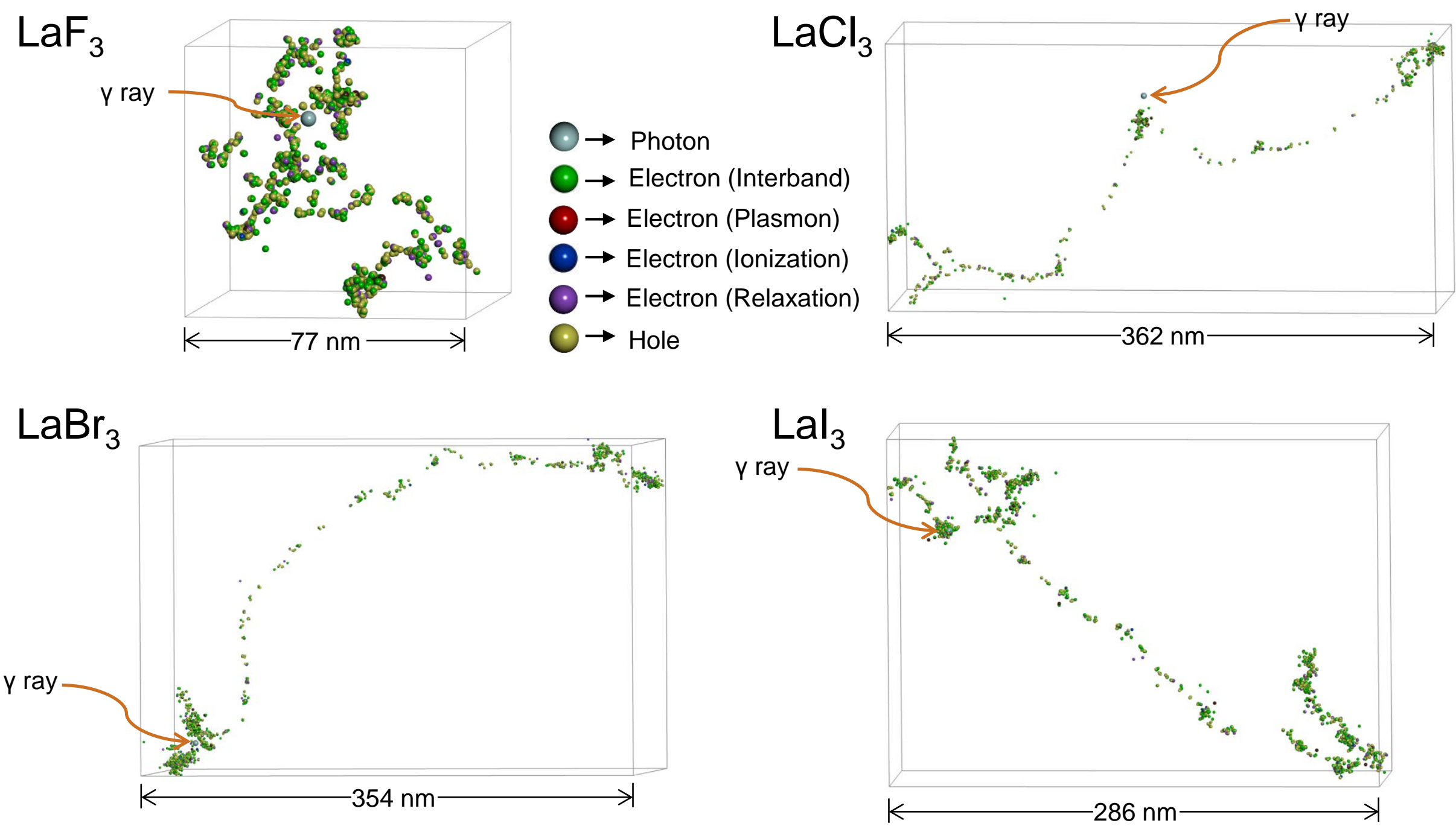

Nano-scale fluctuations in e-h pair density along the track are key to nonproportionality 


\section{Maximum theoretical light yields}

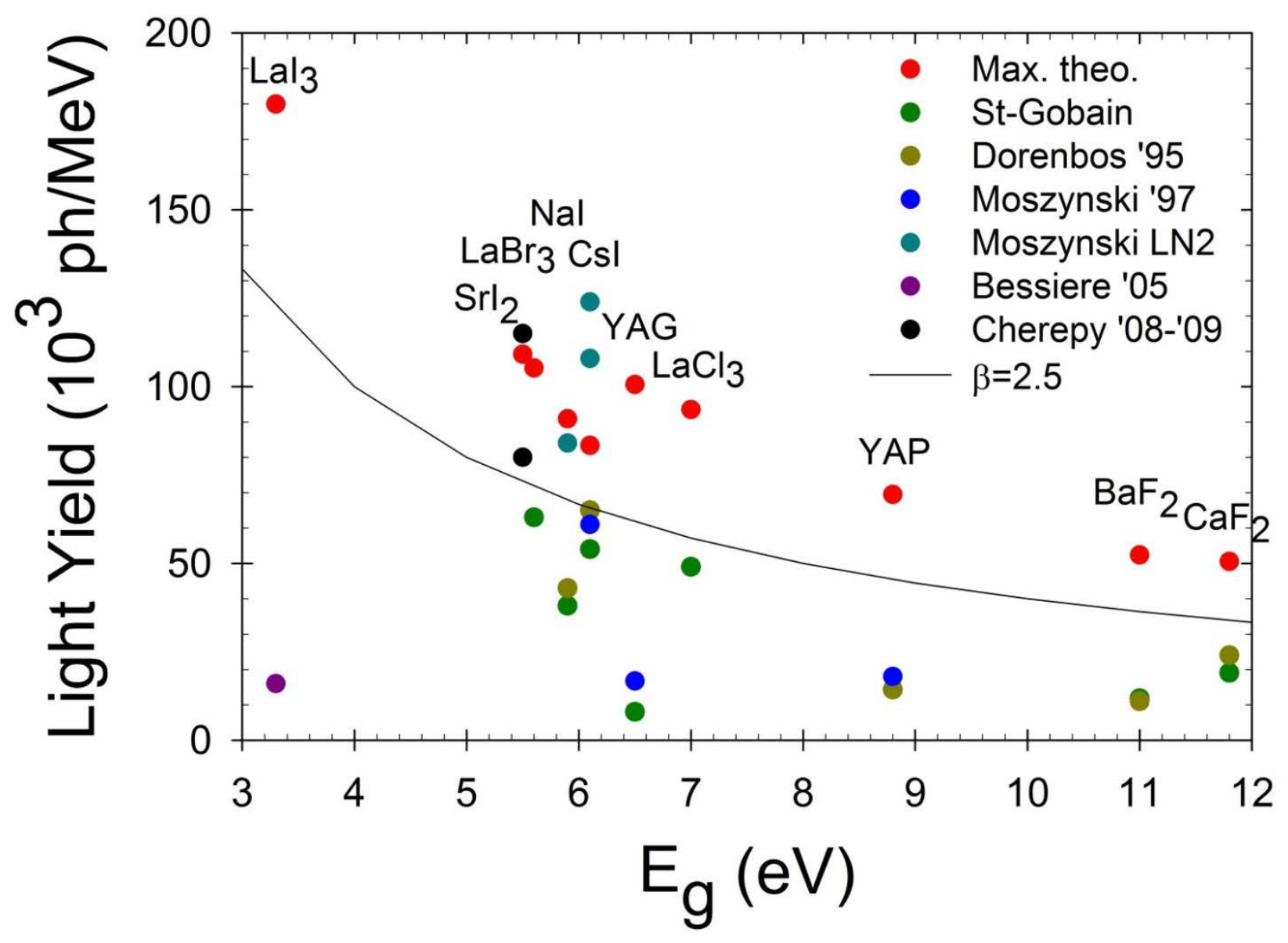

NWEGRIM can be used for rapid evaluation of:

- the potential brightness of a candidate scintillator

- the potential for improvement of current scintillators

- the upper limit for absolute light yields

D. Wu, Y. Xie, F. Gao 


\section{Intrinsic properties}

\begin{tabular}{|c|c|c|c|c|c|c|}
\hline Property & $\mathrm{CaF}_{2}$ & $\mathrm{BaF}_{2}$ & CsI & Nal & $\mathrm{LaBr}_{3}$ & $\mathrm{Srl}_{2}$ \\
\hline $\mathrm{W}(\mathrm{eV})$ & 21.4 & 19.1 & 12.0 & 10.9 & 9.5 & 9.2 \\
\hline$\beta$ & 1.8 & 1.7 & 2.0 & 1.9 & 1.7 & 1.7 \\
\hline Interband & $63.1 \%$ & $63.7 \%$ & $65.2 \%$ & $60.2 \%$ & $72.1 \%$ & $72.2 \%$ \\
\hline Plasmon & $31.6 \%$ & $29.3 \%$ & $18.2 \%$ & $29.1 \%$ & $21.9 \%$ & $19.2 \%$ \\
\hline Ionization & $4.4 \%$ & $4.1 \%$ & $11.1 \%$ & $6.4 \%$ & $3.2 \%$ & $4.3 \%$ \\
\hline Fano factor & 0.23 & 0.19 & 0.30 & 0.28 & 0.21 & 0.23 \\
\hline $\begin{array}{c}\text { Track } \\
\text { structure }\end{array}$ & Clustered & Clustered & $\begin{array}{l}\text { Slightly } \\
\text { clustered }\end{array}$ & $\begin{array}{l}\text { Slightly } \\
\text { clustered }\end{array}$ & Linear & Linear \\
\hline
\end{tabular}

- Small number of energy loss channels (ideally, only interband transitions)

- Linear track structure that decreases nonlinear quenching at low energies

- Correlation between loss channels and Fano factor

D. Wu, Y. Xie, F. Gao

Gao et al. J. Appl. Phys. 114, 173512 (2013)

Gao et al. NIM A 652, 564 (2011) 


\section{Lanthanum halide and elpasolite series}

Lanthanum halide series

\begin{tabular}{|c|c|c|c|c|}
\hline Material & $\begin{array}{c}\text { Band gap } \\
\text { energy (eV) }\end{array}$ & $\begin{array}{c}\text { Max. theo. LY } \\
(\mathbf{p h} / \mathbf{M e V})\end{array}$ & $\boldsymbol{\beta}$ & Fano factor \\
\hline $\mathrm{LaF}_{3}$ & 6.6 & 96,899 & 2.6 & 0.37 \\
\hline $\mathrm{LaCl}_{3}$ & 7.0 & 93,545 & 1.5 & 0.25 \\
\hline $\mathrm{LaBr}_{3}$ & 5.6 & 105,263 & 1.7 & 0.22 \\
\hline $\mathrm{Lal}_{3}$ & 3.3 & 173,913 & 1.7 & 0.52 \\
\hline
\end{tabular}

- On-going ab initio calculations to search for suitable activators for $\mathrm{Lal}_{3}$ (e.g. Pr, Nd, Eu, etc)

Elpasolite series - dual gamma/neutron detectors

\begin{tabular}{|c|c|c|c|c|}
\hline Material & $\begin{array}{c}\text { Band gap } \\
\text { energy (eV) }\end{array}$ & $\begin{array}{c}\text { Max. theo. LY } \\
\text { (ph/MeV) }\end{array}$ & $\boldsymbol{\beta}$ & Fano factor \\
\hline $\mathrm{Cs}_{2} \mathrm{LiLaBr}_{6}$ & 6.0 & 103,093 & 1.6 & 0.35 \\
\hline $\mathrm{Cs}_{2} \mathrm{LiLaCl}_{6}$ & 8.0 & 67,431 & 1.8 & 0.24 \\
\hline $\mathrm{Cs}_{2} \mathrm{LiYBr}_{6}$ & 5.7 & 105,374 & 1.7 & 0.32 \\
\hline $\mathrm{Cs}_{2} \mathrm{LiYCl}_{6}$ & 7.5 & 69,686 & 1.9 & 0.27 \\
\hline
\end{tabular}

- NWEGRIM for neutron response + KMC nonlinear quenching $\rightarrow$ Pulse shape discrimination 


\section{Electron thermalization}

A Monte Carlo model of electron thermalization was developed and implemented:

- Uses as input spatial and kinetic energy distributions of e-h pairs from NWEGRIM

- Scattering with longitudinal optical (Frölich) and acoustic (deformable pot.) phonons

- Includes the effects of internal electric fields

- Applied to alkali/alkaline-earth halides ( $\mathrm{Csl}, \mathrm{Nal}, \mathrm{CaF}_{2}, \mathrm{BaF}_{2}$ )

\section{Key findings and impact}

- Quantified time (ps) and length (10's to 100's nm) scales of thermalization

- Slow electron cooling significantly affects density in the track

- Allowed development of improved conceptual models

e.g. Scintillator "Decision Tree" (WFU) Li et al. PSS RRL 6, 346 (2012)

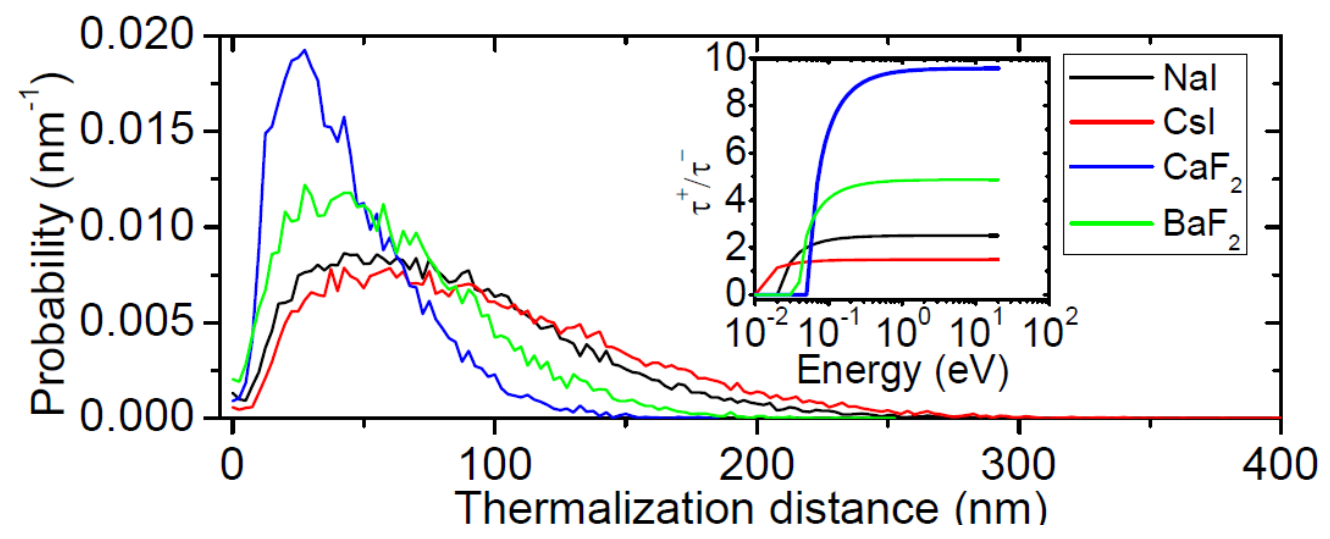

Z. Wang, S. Kerisit

Wang et al. J. Appl. Phys. 110, 064903 (2011)

Wang et al. J. Appl. Phys. 112, 014906 (2012)

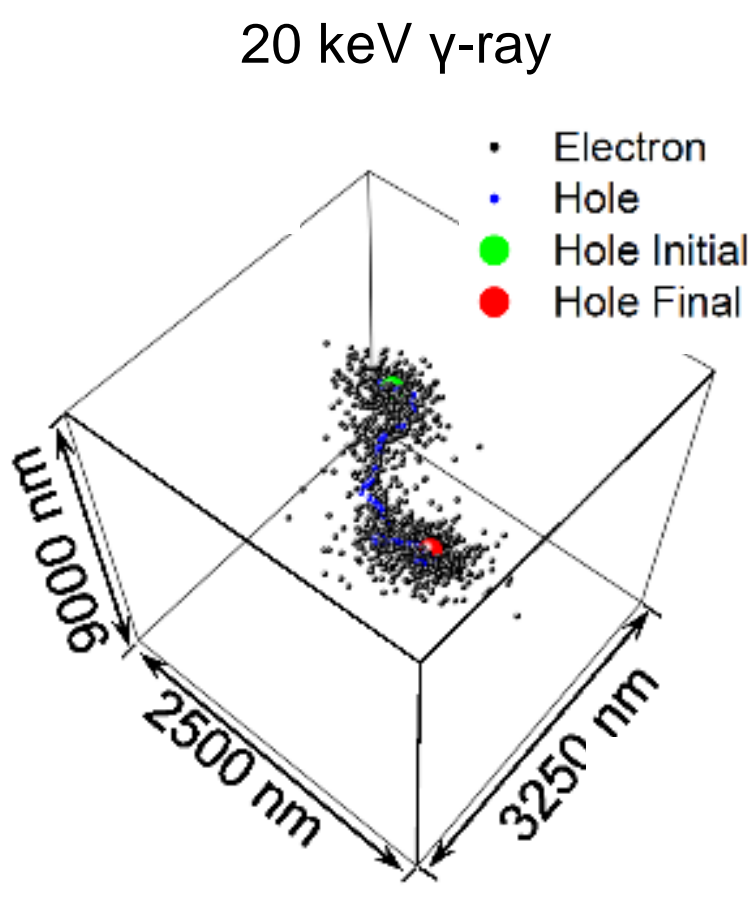




\section{$A b$ initio calculations of vibrational excitations}

- Accurate treatment of thermalization is necessary for quantitative prediction of scintillator performance.

- Existing approaches to electron-phonon interaction are insufficient for electron cascades which contain very hot carriers.

- Direct ab initio methods: no empirical inputs or model interaction Hamiltonians (e.g. deformation potential, Frölich model, etc).

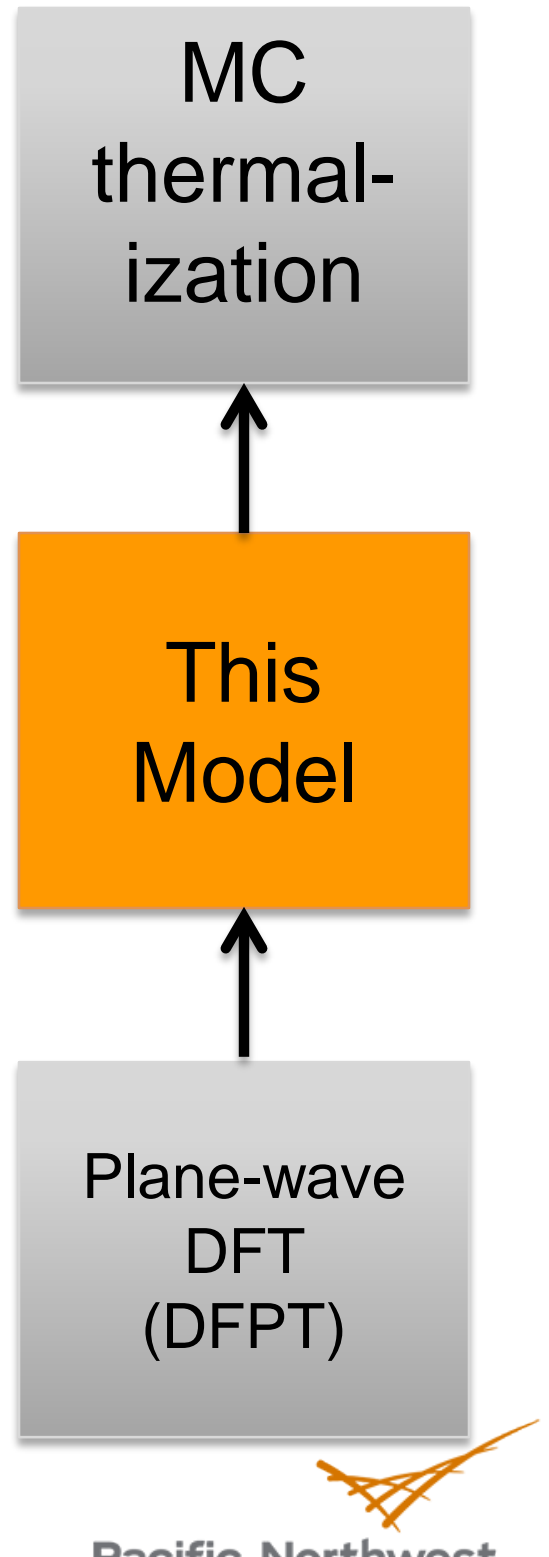




\section{"Golden Rule" for energy loss rate}

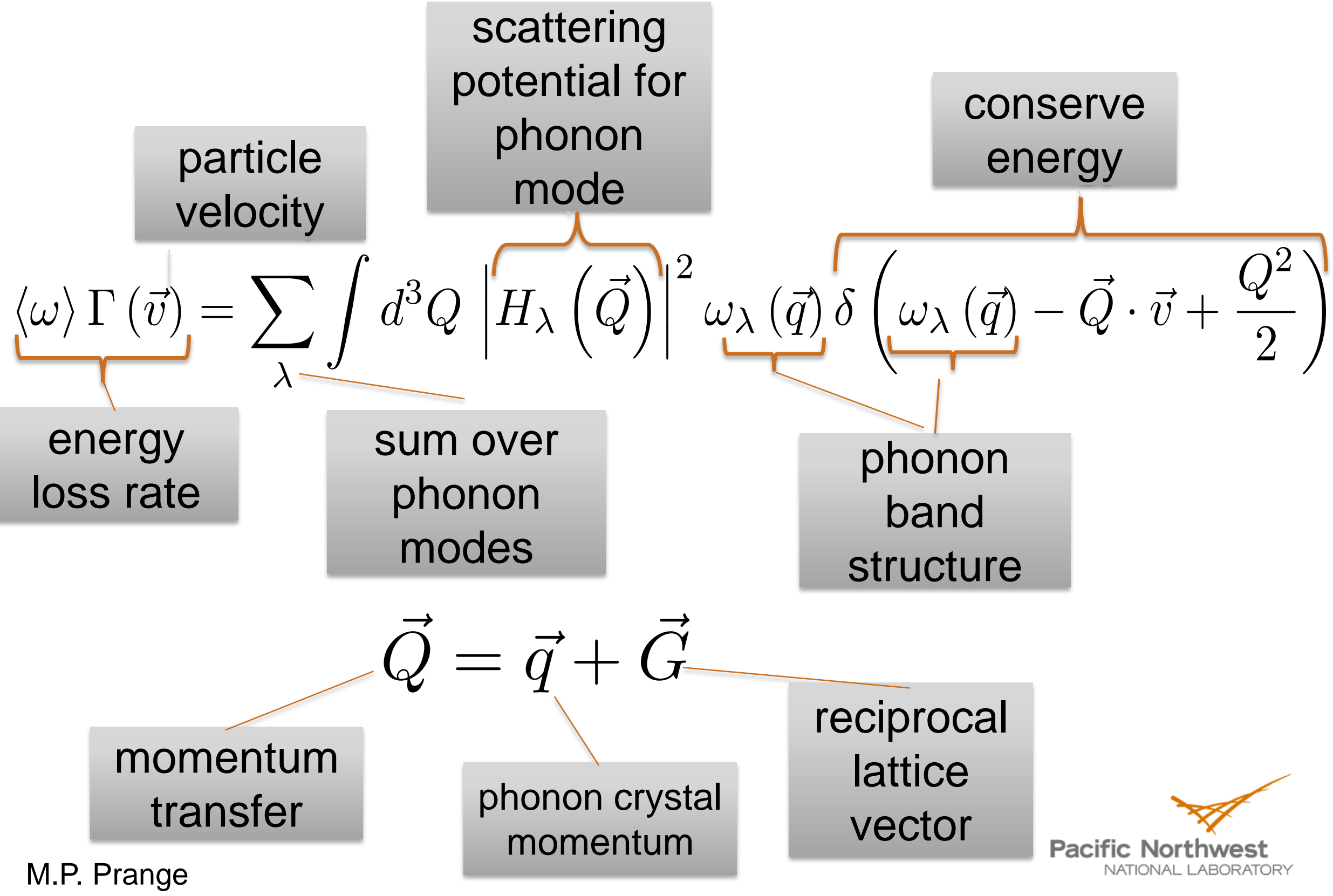




\section{Energy loss rates in CsI}

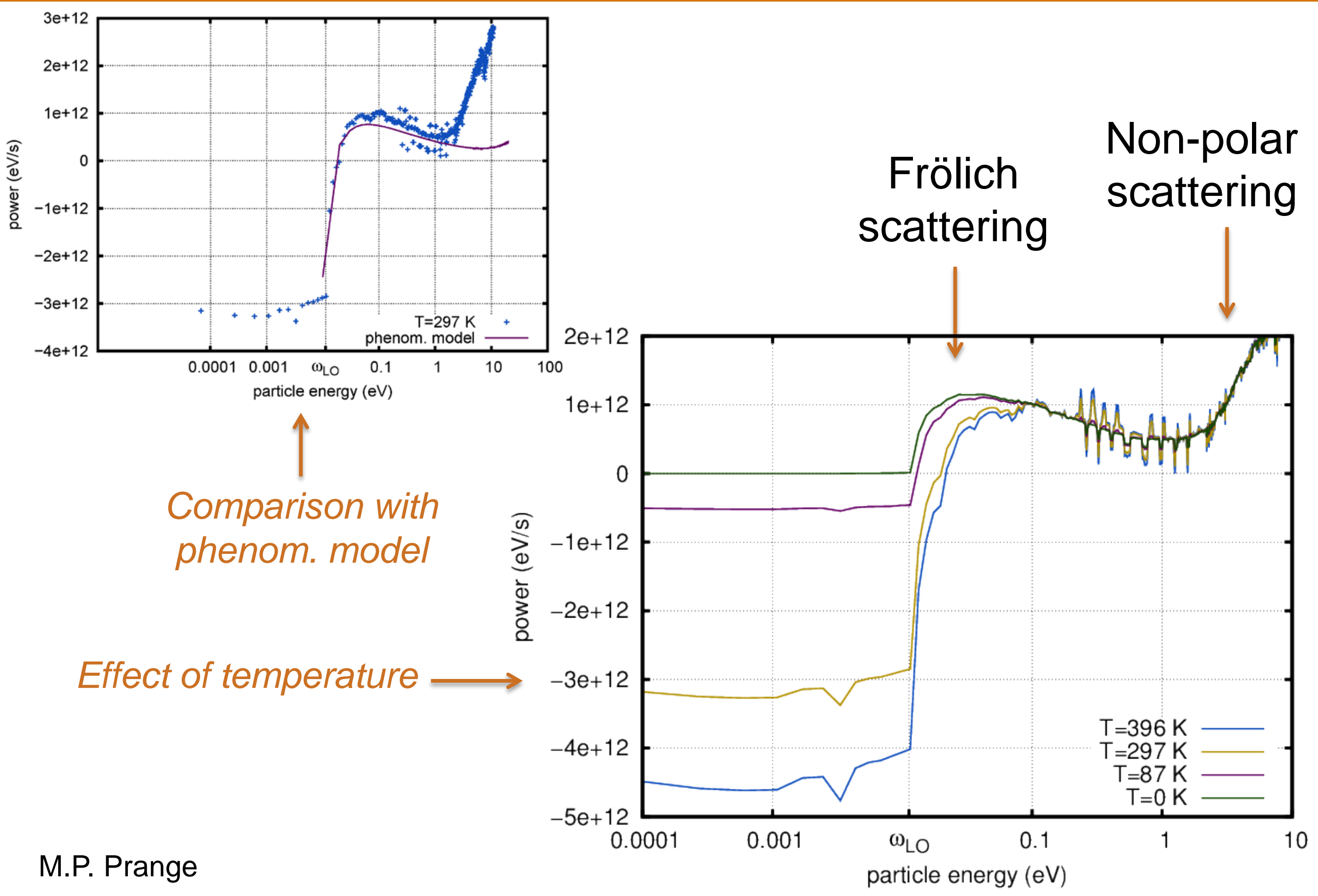




\section{Kinetic Monte Carlo (KMC) model of scintillation processes}

- Atomic-level simulation of the kinetics and efficiency of scintillation processes

- A wide range of mechanisms can be simulated

Pre-exponential factor, $\mathrm{A}$, and activation energy, W, needed to parameterize each process.

$$
k=A \times \exp \left(-W / k_{\mathrm{B}} T\right)
$$

- KMC simulations allow for explicit representation of inherent heterogeneous nature of ionization tracks

- Nonlinear quenching (in collaboration with WFU)

Use data measured under simpler conditions than a $y$ ray track to isolate specific properties

Z-scan/photon response data for UV-excited Nal(TI) and

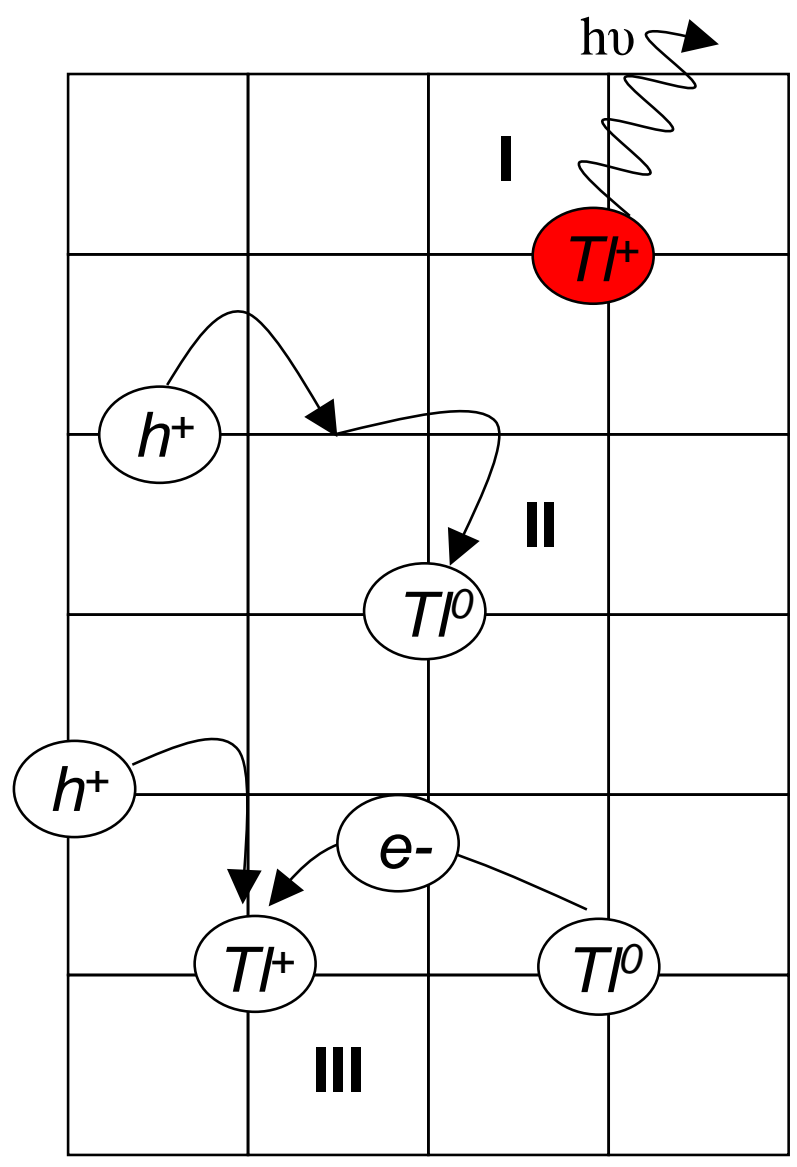

$\mathrm{CsI}(\mathrm{TI})(5.9 \mathrm{eV})$ obtained by Williams and Grim at WFU 


\section{STE-STE annihilation}

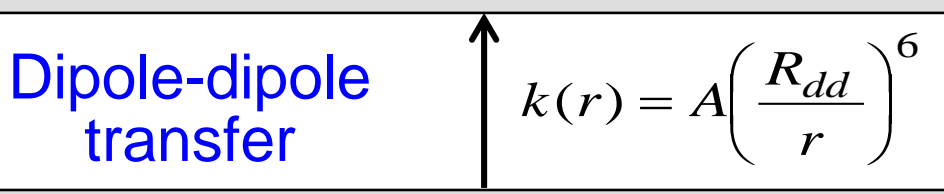

\section{STE luminescence}

\section{Self-trapped Exciton (STE)}

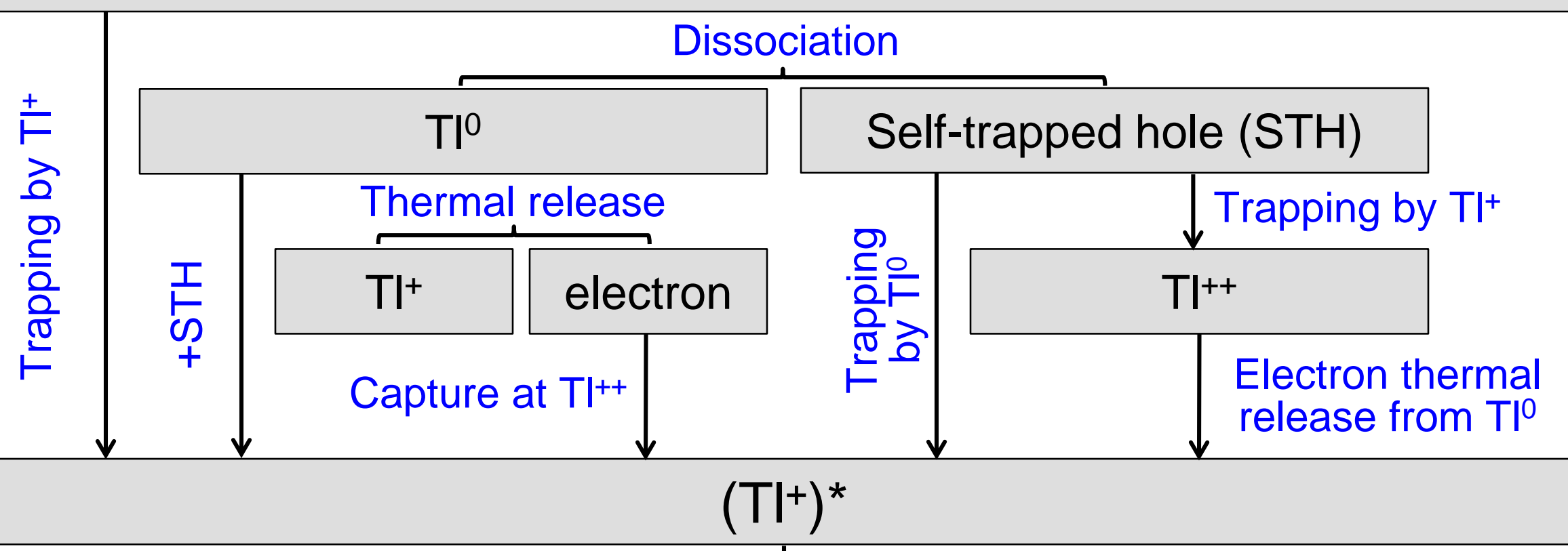

Radiative decay

Thallium luminescence 


\section{Ab initio calculations of information carriers}
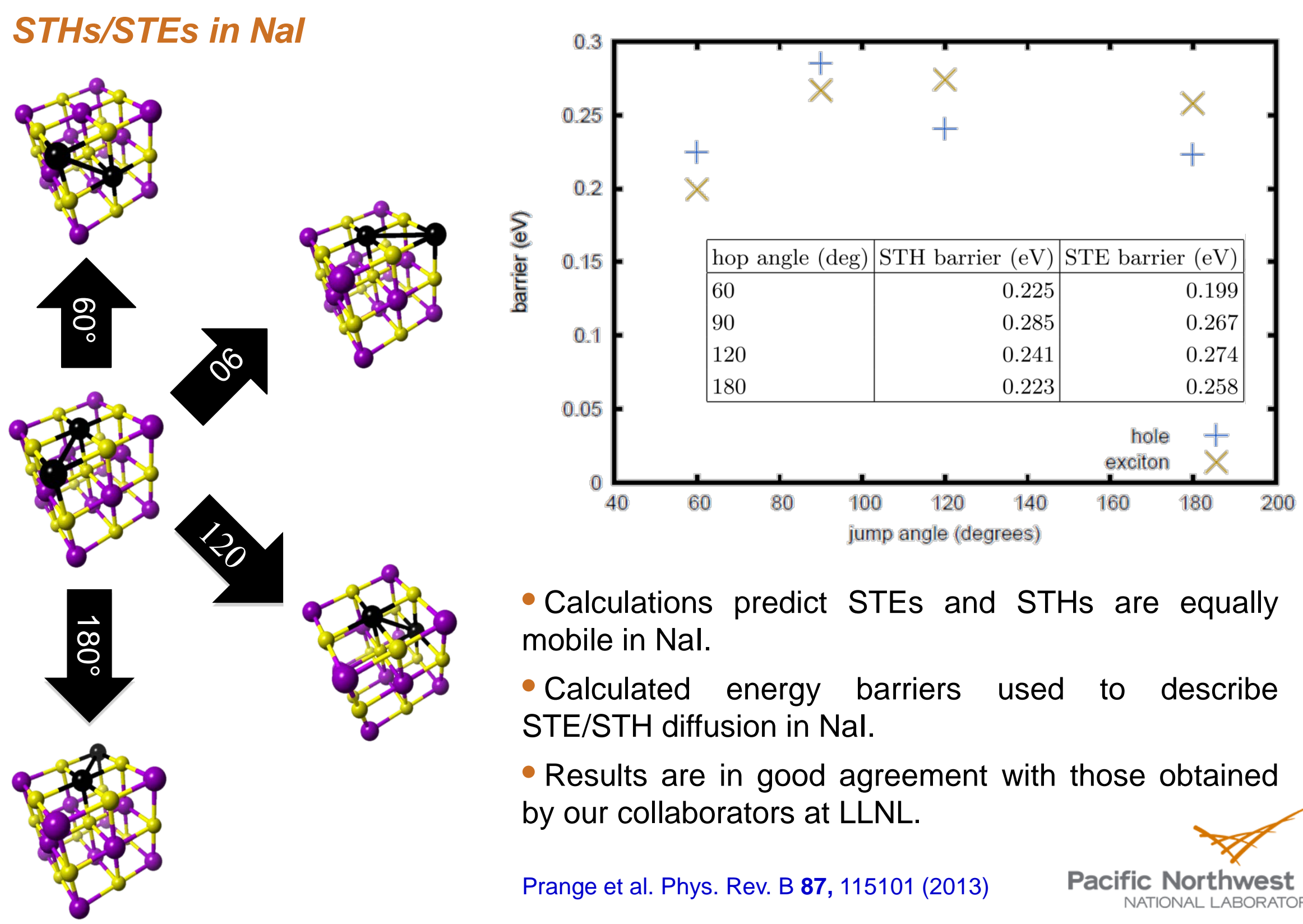

- Calculations predict STEs and STHs are equally mobile in Nal.

- Calculated energy barriers used to describe STE/STH diffusion in Nal.

- Results are in good agreement with those obtained by our collaborators at LLNL.

Prange et al. Phys. Rev. B 87, 115101 (2013) 


\section{Scintillation efficiency}

\section{Nal:0.1 mol\% TI - 5.9 eV excitation}

Kerisit et al. IEEE Trans. Nucl. Sci. (2014) in press
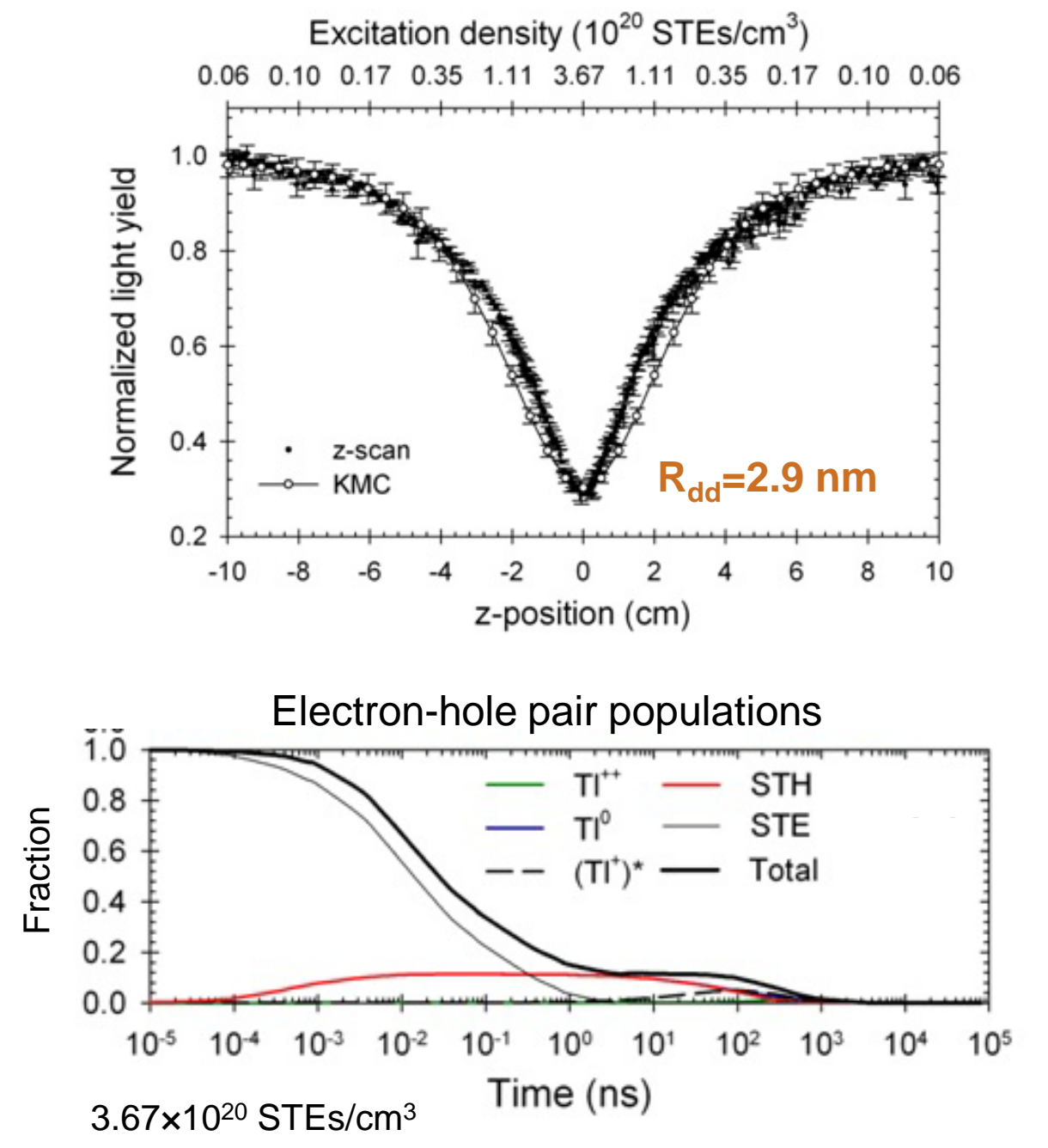

Csl:0.3 mol\% TI - 5.9 eV excitation

Wang et al. Phys. Status Solidi B 250, 1532 (2013)

\section{Excitation density $\left(10^{20} \mathrm{STEs} / \mathrm{cm}^{3}\right)$}
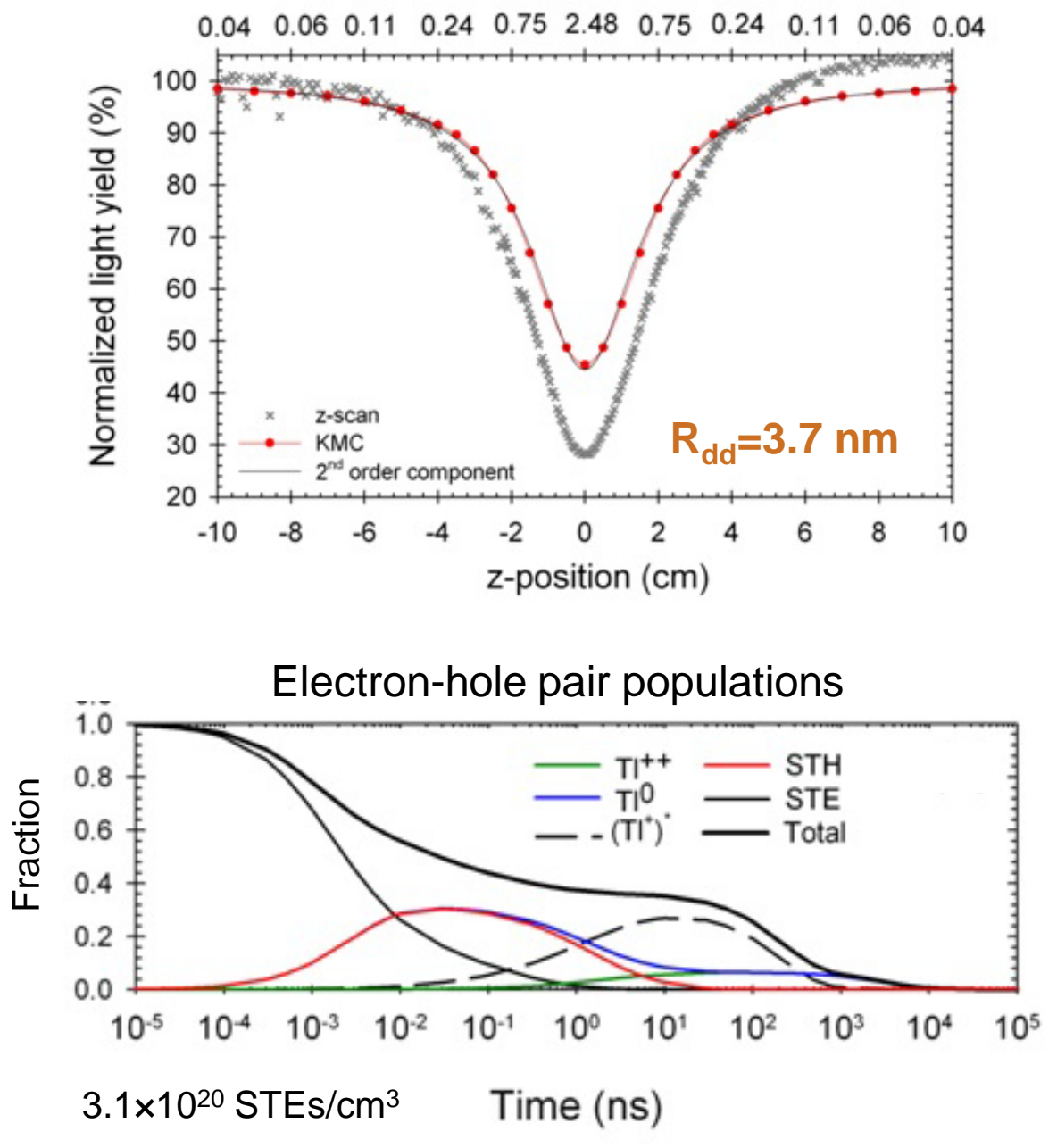

Exp. data from Grim et al. Phys. Rev. B 87, 125117 (2013) 


\section{Scintillation kinetics}

Csl:0.3 mol\% Tl - 5.9 eV excitation

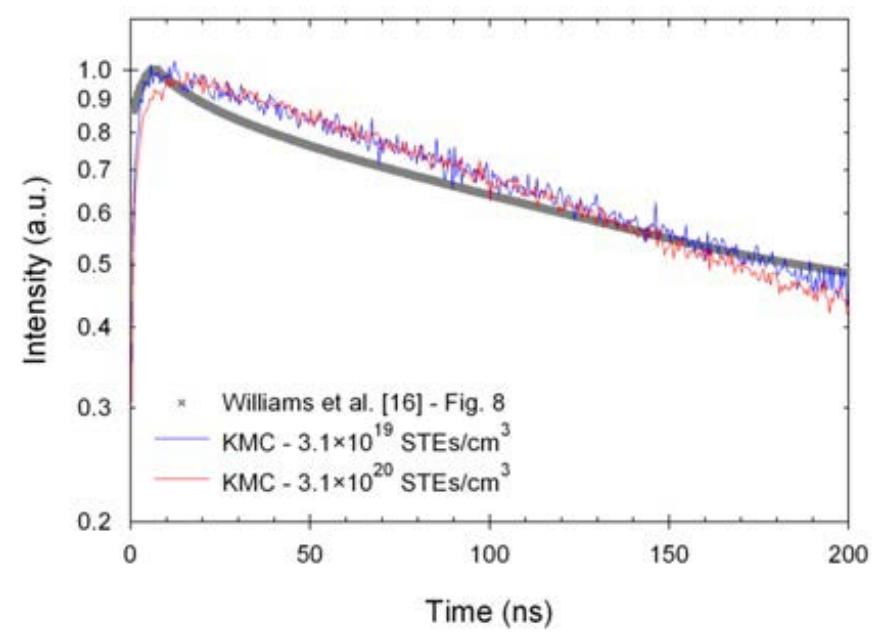

Pure CsI - 5.9 eV excitation

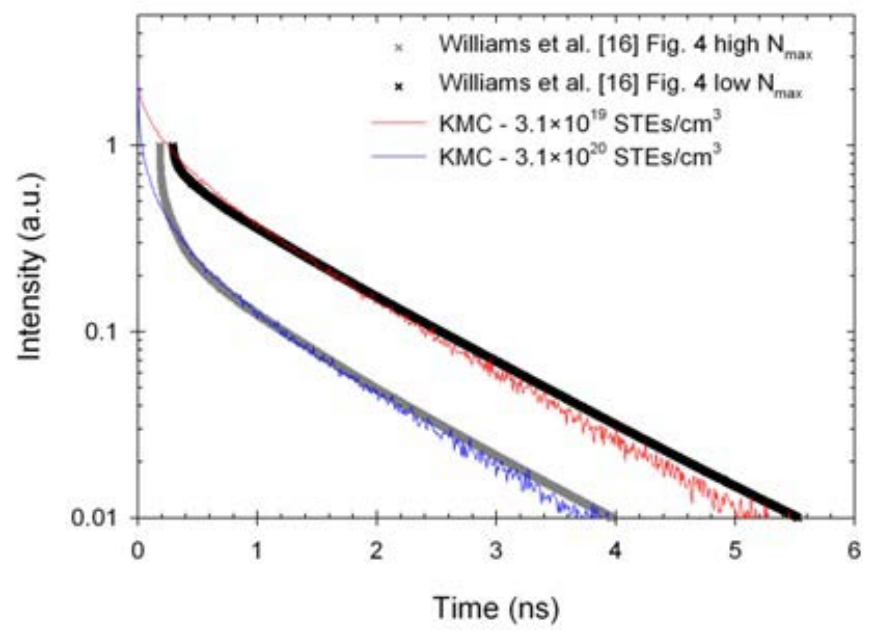

Exp. data from Williams et al. PSSB 248, 426 (2011)
Csl:0.1 mol\% TI - 511 keV y-rays

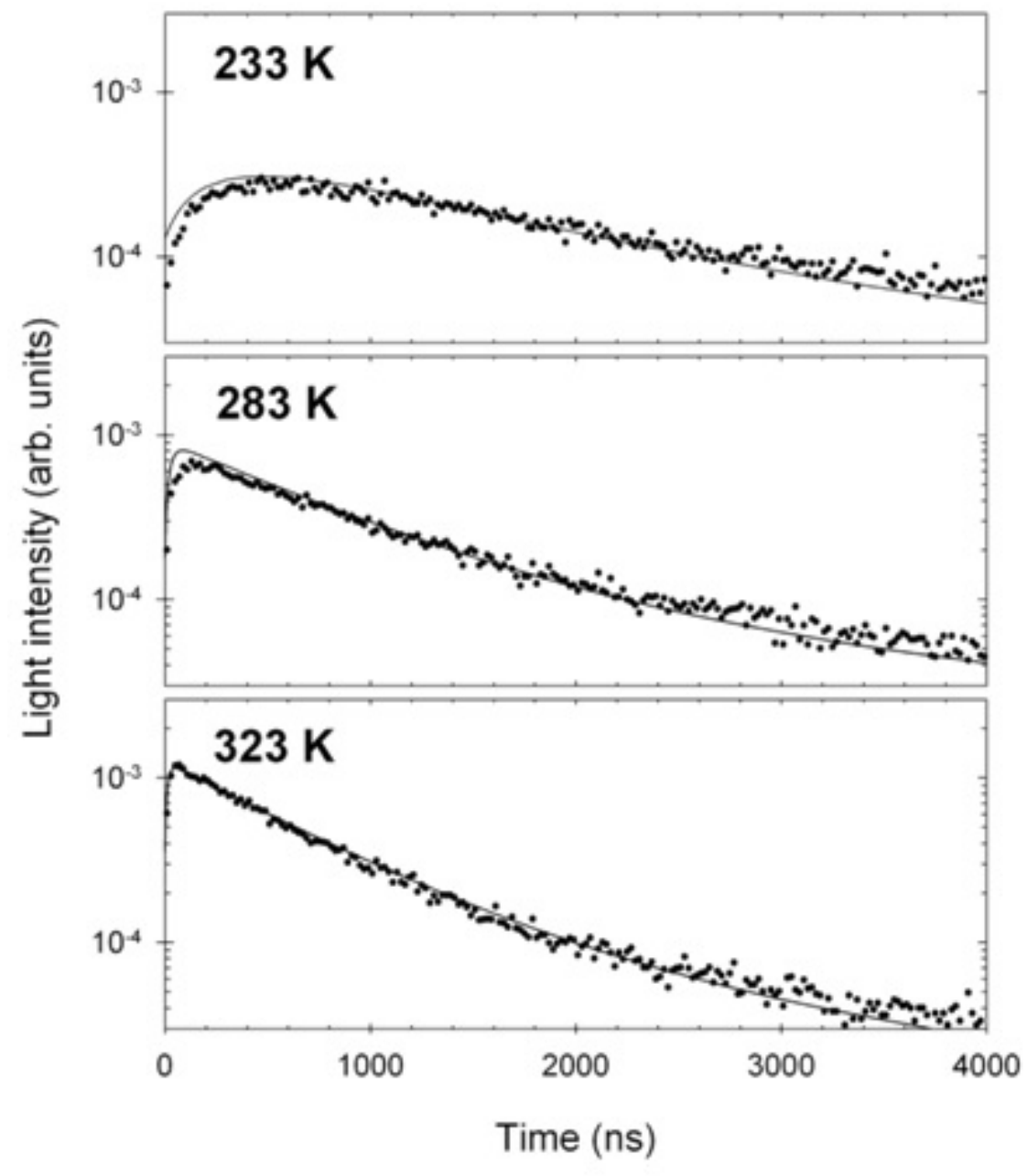

Exp. data from Valentine et al. NIM A 325, 147 (1993) 


\section{Summary \& Outlook}

\section{Where we are}

- ERSP: ab initio electron cross sections

- NWEGRIM: $y$-ray and electron energy cascades

- DefDen: ab initio electron-phonon scattering in model system

- KMC: kinetics and efficiency including $2^{\text {nd }}$-order quenching

- Merge thermalization and KMC code

\section{Remainder of FY14 and FY15}

- ERSP: extend and complete heavy particle work

- NWEGRIM: extend to other particles $\rightarrow$ pulse shape discrimination

- DefDen: extend to $\mathrm{Srl}_{2}$ and other scintillator materials

- KMC: complete $3^{\text {rd }}$-order quenching work + apply to tracks $\rightarrow$ nonproportionality 


\section{Radiation response of inorganic scintillators: Insights from Monte Carlo simulations}

\section{Sebastien Kerisit, Micah P. Prange, Dangxin Wu, Yulong Xie, Luke W. Campbell, and Fei Gao}

Pacific Northwest National Laboratory

This project is funded by the National Nuclear Security Administration, Office of Defense Nuclear Nonproliferation, Office of Nonproliferation and Validation Research \& Development (DNN R\&D/NA-22), U.S. Department of Energy.

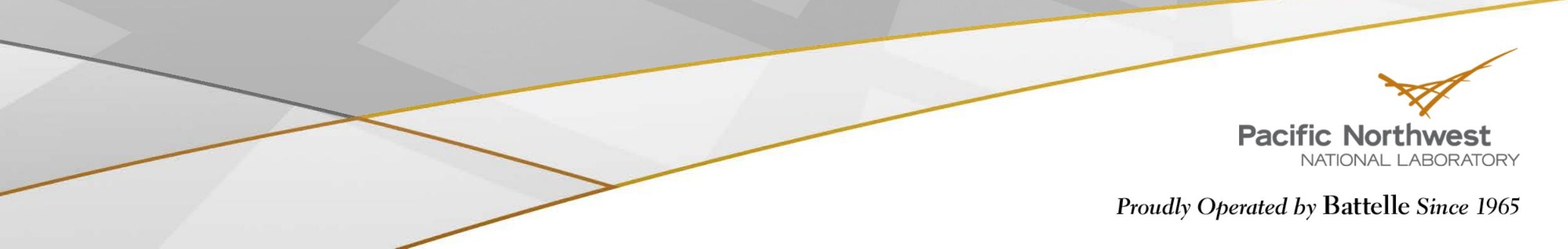




\section{Motivation \& Objective}

\section{Motivation}

- Why some scintillator materials offer better performance than others? (e.g. nonproportionality)

- What are the fundamental performance limits of a given scintillator material?

- Fundamental physical processes that control scintillator performance are not well understood.

- Need for developing comprehensive scintillator physics models/computer codes.

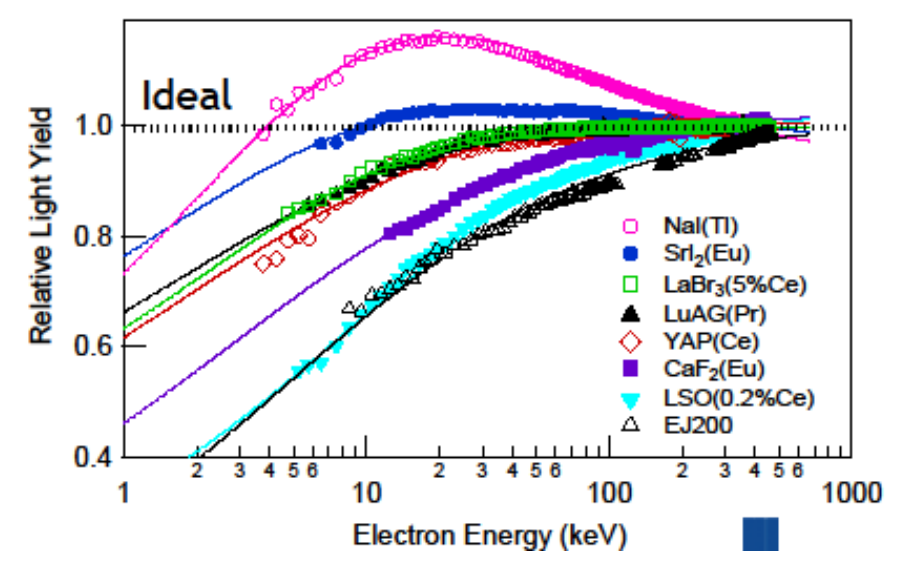

S. Payne - SPIE meeting 2011

\section{Objective}

\section{Develop predictive models to understand radiation response}

Thereby providing a theoretical basis to:

- Improve the performance of current scintillators

- Accelerate the candidate search for new scintillator materials 


\section{Overview of Approach}

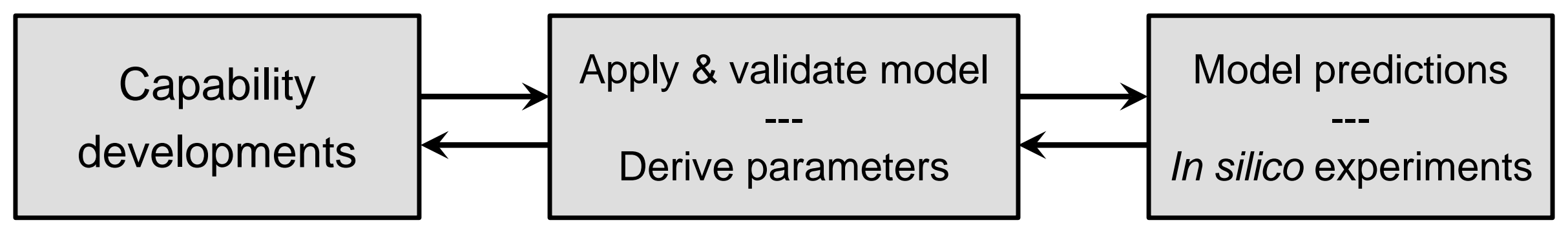

\section{Capability developments}

- Electronic ReSPonse (ERSP)

Low-energy cross sections and plasmon decay spectra

- NorthWest Electron \& Gamma-Ray Interaction with Matter (NWEGRIM)

Energy cascade $\rightarrow$ generation of electron-hole pairs

- Deformable Density (DefDen) model

Electron/hole-phonon interactions

- Kinetic Monte Carlo (KMC) code

Carrier thermalization, transport, recombination, and luminescence

\section{Model applications and predictions}

- Wide range of scintillators (alkali/alkaline-earth/lanthanum halides, oxides, elpasolites)

- Light yields, decay kinetics, nonproportionality, pulse shape discrimination

- Establish rules that dictate scintillator performance (self-consistent analysis)

- Material engineering (co-doping, defect manipulation) 

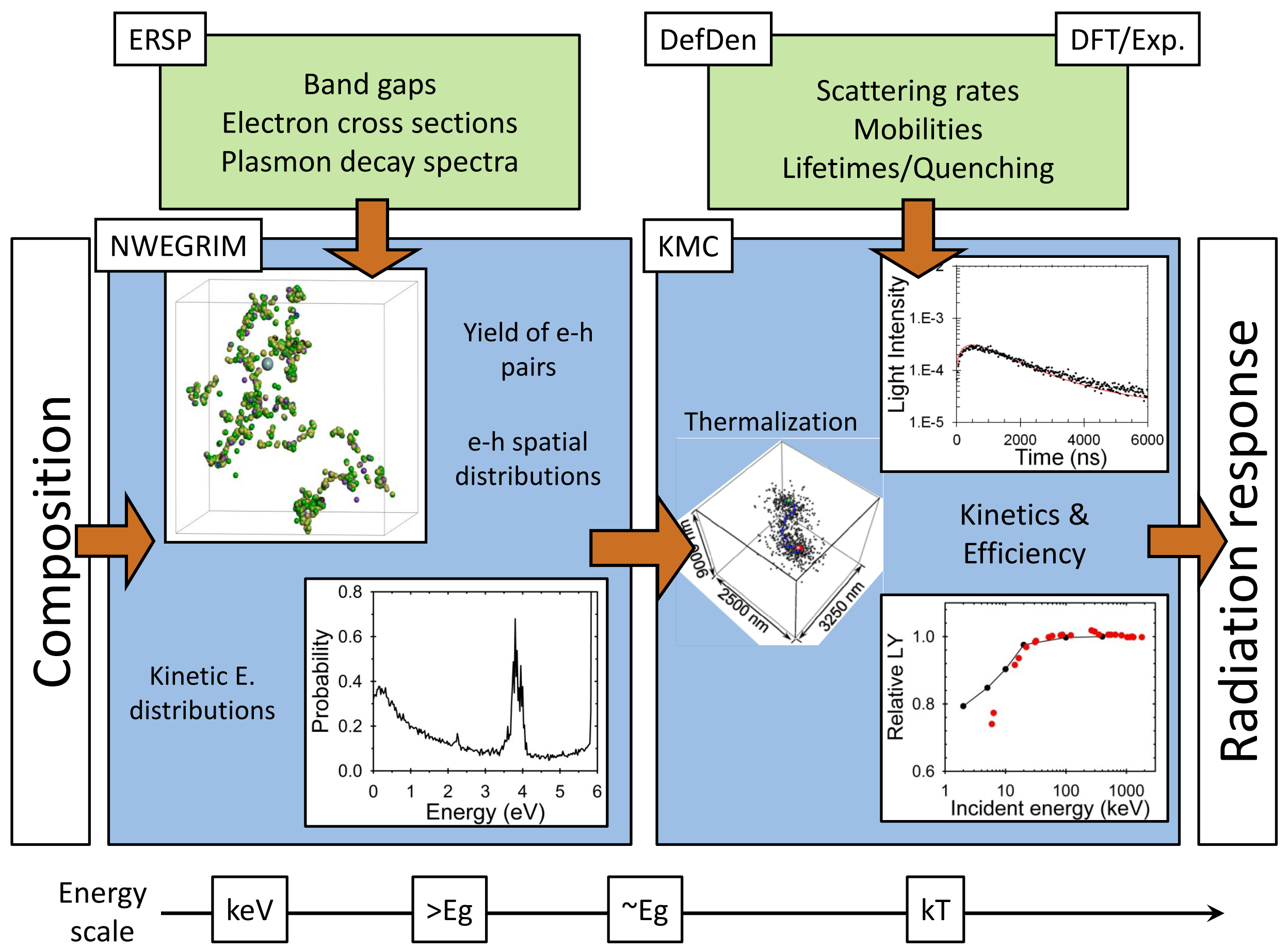


\section{Thermalization: approach}

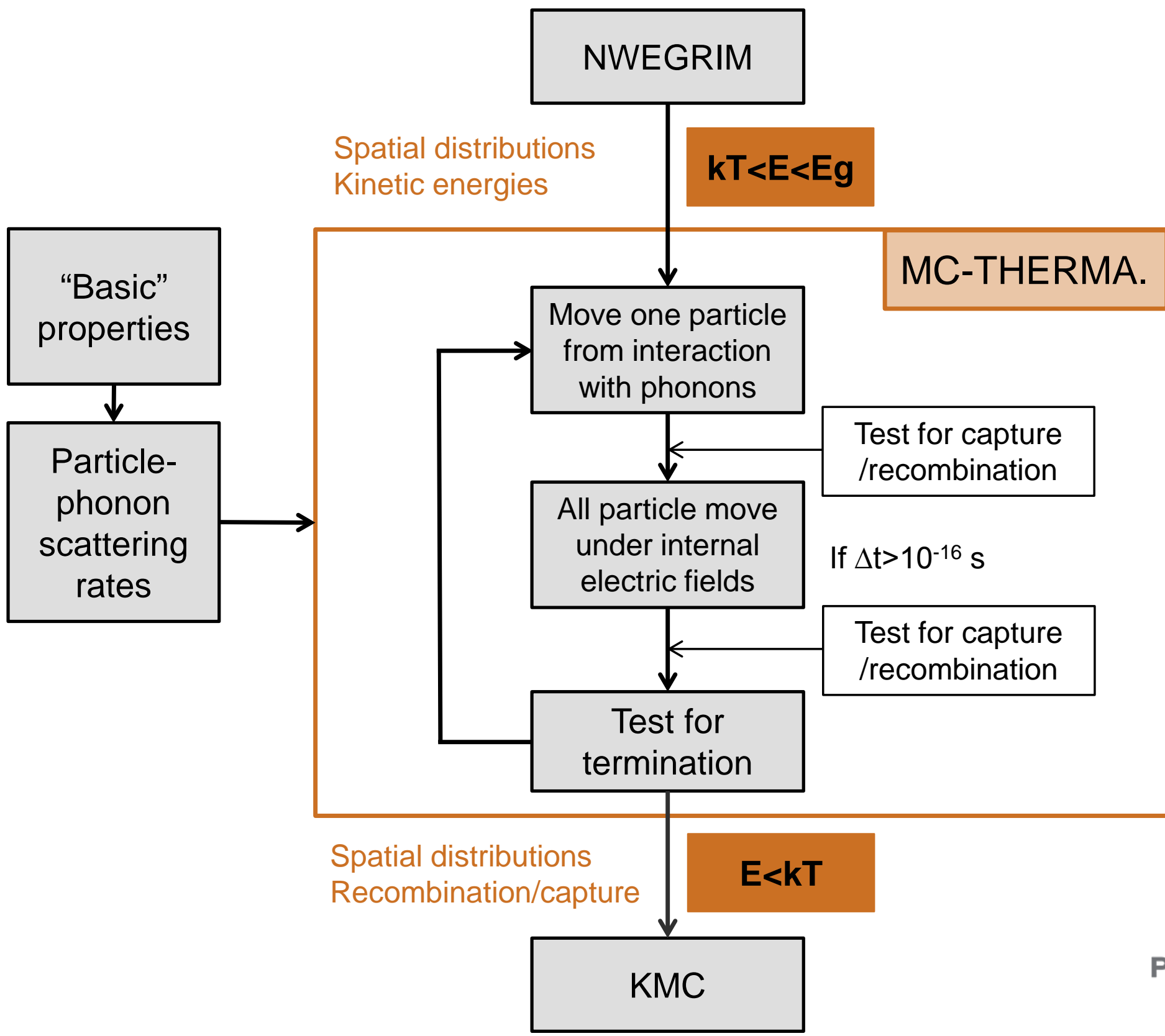




\section{Particle-phonon interactions}

\section{Particle-LO phonon scattering rate}

Scattering rates and angles for emission and absorption of LO phonons are calculated using the formulation of Llacer and Garwin 1 .

$$
\tau_{L O}^{ \pm}=\frac{1}{4 \pi \varepsilon} \frac{e^{2}}{v}\left(n_{q}+\frac{1}{2} \pm \frac{1}{2}\right) \frac{\omega_{o p t}}{\hbar}\left(\frac{1}{\varepsilon_{\infty}}-\frac{1}{\varepsilon_{0}}\right) \ln \left(\frac{\left.1+\sqrt{1 \mp\left(\hbar \omega_{o p t} / E\right.}\right)}{\left.1-\sqrt{1 \mp\left(\hbar \omega_{o p t} / E\right.}\right)}\right)
$$

where $\varepsilon$ is the vacuum permittivity, $e$ is the elementary charge, $v$ is the particle speed, $\hbar$ is the Planck constant, $\omega_{\text {opt }}$ is the LO phonon angular frequency, $E$ is the particle kinetic energy, $n_{q}$ is the phonon occupation number, $\varepsilon_{\infty}$ and $\varepsilon_{0}$ are the optical and static dielectric constants.

\section{Particle-A phonon scattering rate}

Scattering rates and angles for emission and absorption of acoustic phonons are calculated using the formulation of Sparks et al. ${ }^{2}$ together with the correction of Bradford and Woolf ${ }^{3}$.

\section{Primary parameters}

Lattice parameter, dielectric constants, elastic constants

LO characteristic phonon energy, elastic scattering cross section

1J. Llacer and E.L. Garwin (1969) J. Appl. Phys. 402766

${ }^{2}$ M. Sparks et al. (1981) Phys. Rev. B 243519 


\section{Internal electric fields}

As particles thermalize via interaction with lattice phonons, they are also influenced by the electric field due to all the other electrons and holes generated during the energy cascade.

The particle trajectories were calculated using the classical equations of dynamics:

$$
\frac{d}{d t}\left[m^{*} v_{i}\right]=-e E_{i}
$$

where $m^{*}$ is the particle effective mass, $v_{i}$ the velocity of particle $i$, and $\mathrm{E}_{i}$, the electric field at the position of particle $i$, is defined as:

$$
E_{i}=\sum_{\substack{j=1 \\ j \neq i}}^{N} \frac{1}{4 \pi \varepsilon_{0}} \frac{q_{j}}{r_{i j}^{2}} \hat{r}_{i j}
$$

where $N$ is the number of electrons and holes, $\varepsilon_{0}$ the material's dielectric constant, $q_{j}$ the charge of particle $j, r_{i j}$ the distance between particles $i$ and $\mathrm{j}$, and $\hat{r}_{i j}$ the unit vector.

Equation (1) needs to be discretized using a time step $\Delta \mathrm{t}$. 


\section{Models of quasi-particle dispersion}

Two models for describing quasi-particle dispersion are investigated:

(1) Effective mass approximation (EMA) model

Uses band particle mass calculated from band structure

Polaron effects are taken into account using Fröhlich's theory

$$
\begin{aligned}
& v(E)=\sqrt{\frac{2 E}{m^{*}}} \\
& \frac{m^{*}}{m_{b}}=1+\frac{e}{6 \hbar} \frac{1}{4 \pi \varepsilon} \sqrt{\frac{m_{b}}{2 \hbar \omega_{\text {opt. }}}}\left[\frac{1}{\varepsilon_{\infty}}-\frac{1}{\varepsilon_{0}}\right]
\end{aligned}
$$

(2) Group velocity (GV) model

A wave packet of energy $E$ is assumed to move with speed

$$
v(E)=\frac{\sum_{n} \int_{B Z} d^{3} k\left|\frac{d}{d \vec{k}} E_{n}(\vec{k})\right| \delta\left(E-E_{n}(\vec{k})\right)}{\sum_{n} \int_{B Z} d^{3} k \delta\left(E-E_{n}(\vec{k})\right)}
$$

in the direction of its crystal momentum, $\vec{k}$ Includes contributions from all possible quasi-particle states of energy $\mathrm{E}$ 


\section{Models of quasi-particle dispersion}

\section{Csl band structure and density of states}

Local-density approximation exchange-correlation potential

Band masses are $0.31 m_{0}$ and $2.27 m_{0}$ for holes and electrons, respectively.

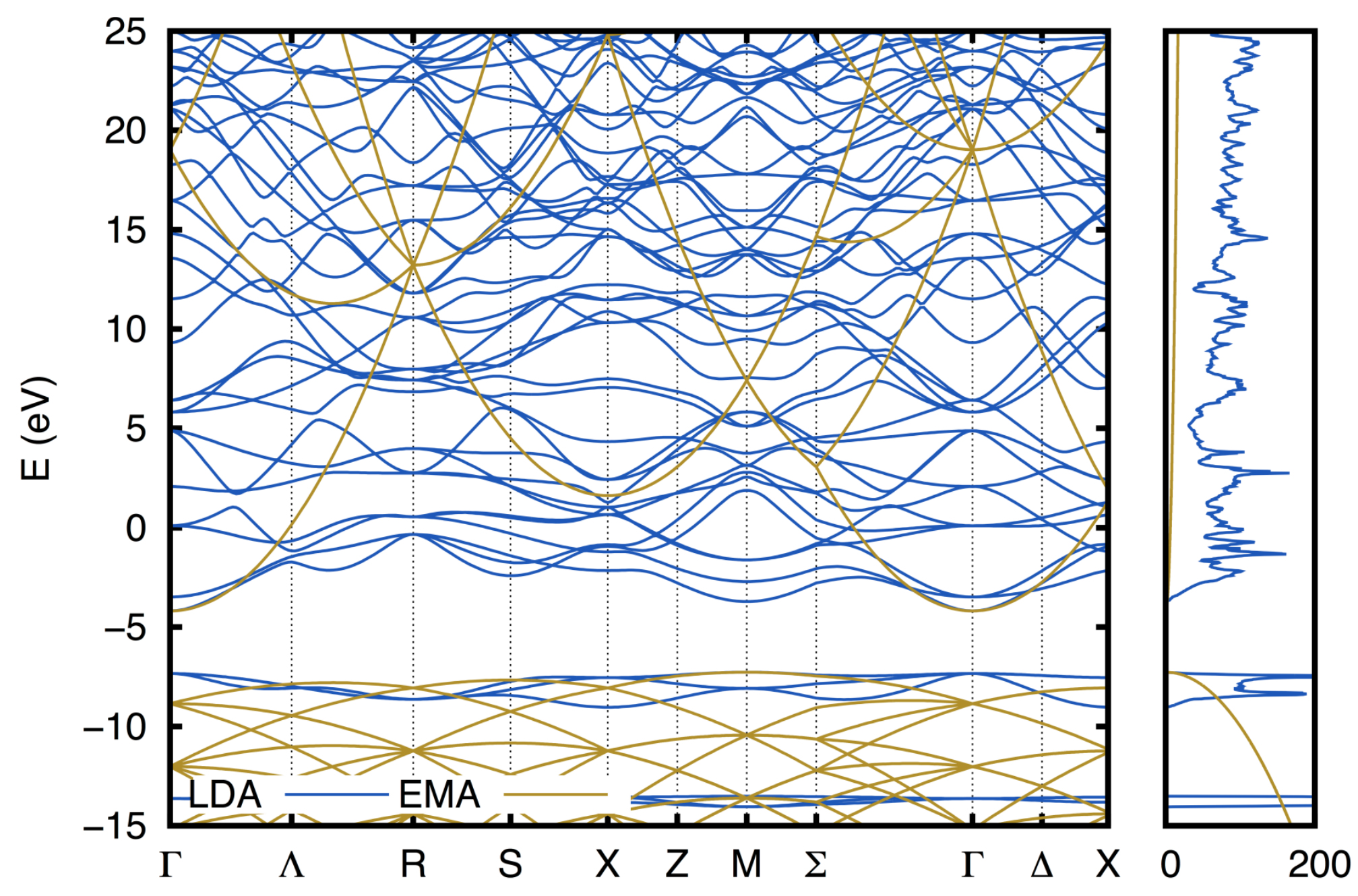




\section{Models of quasi-particle dispersion}

\section{Csl average of the magnitude of the group velocity}

Effective mass approximation gives a good description near edges of the band gap.

But it overestimates speeds at higher energies with respect to group velocities.

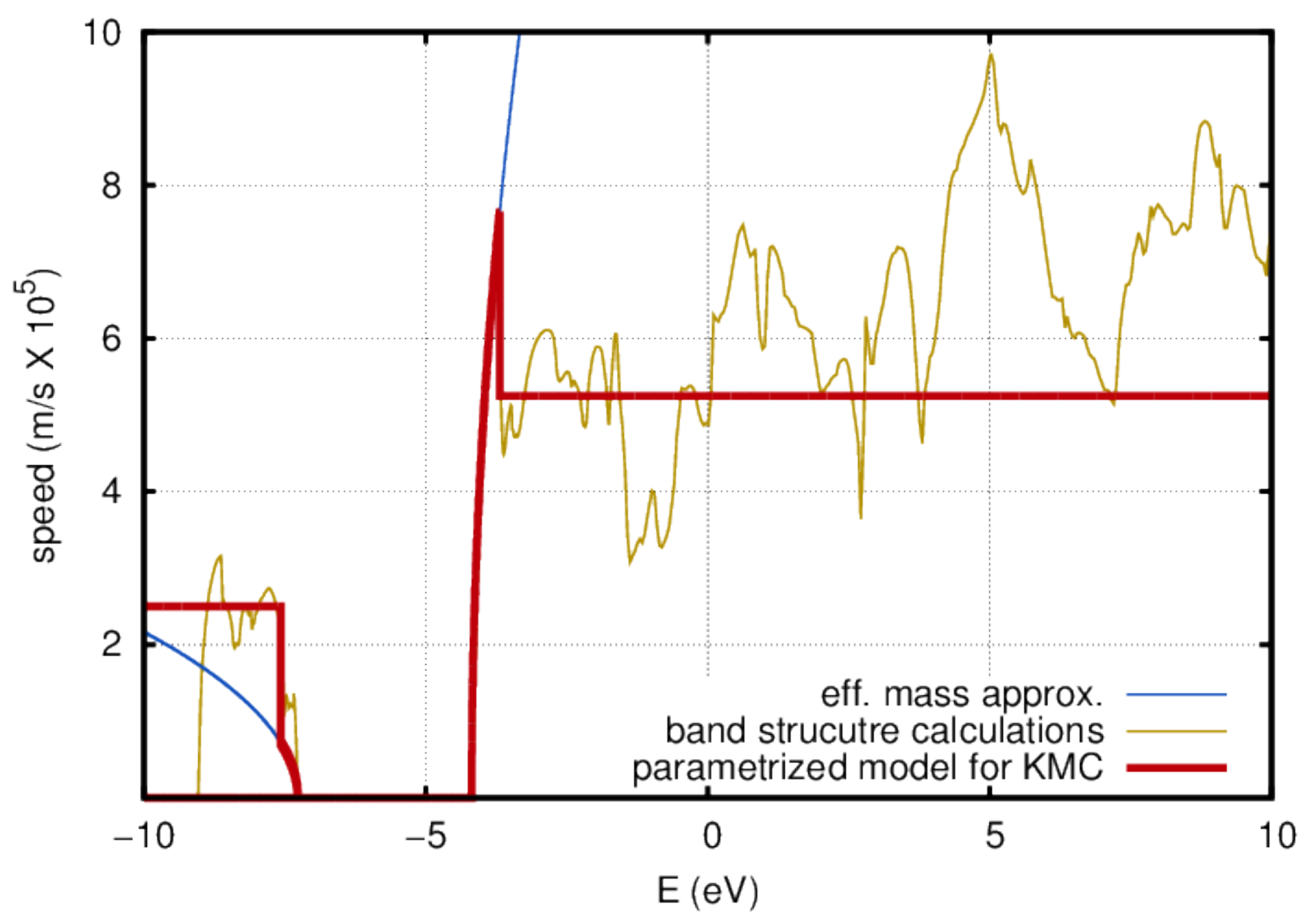




\section{Models of quasi-particle dispersion}

\section{Evaluation of quasi-particle dispersion models}

Picosecond optical absorption study of Williams and co-workers

Pump-probe technique used to excite $\mathrm{Csl}$ by two-photon absorption: 5.9 and $8.86 \mathrm{eV}$

$\mathrm{Csl}$ band gap energy $=5.8-5.9 \mathrm{eV} \rightarrow$ free electrons with $\mathrm{KE}$ of 0.1 and $3.0 \mathrm{eV}$

Time-resolved picosecond spectroscopy of optical absorption:

4 ps delay in onset of self-trapped exciton formation at $3.0 \mathrm{eV}$ vs. $0.1 \mathrm{eV}$

Thermalization time distributions

Two simulations performed for each model with initial KE of 0.1 and $3.0 \mathrm{eV}$ $10^{18}$ electron-hole pairs per $\mathrm{cm}^{3}$

GV model: 3.4 ps delay EMA model: $\sim 9$ ps delay

GV model yields better agreement

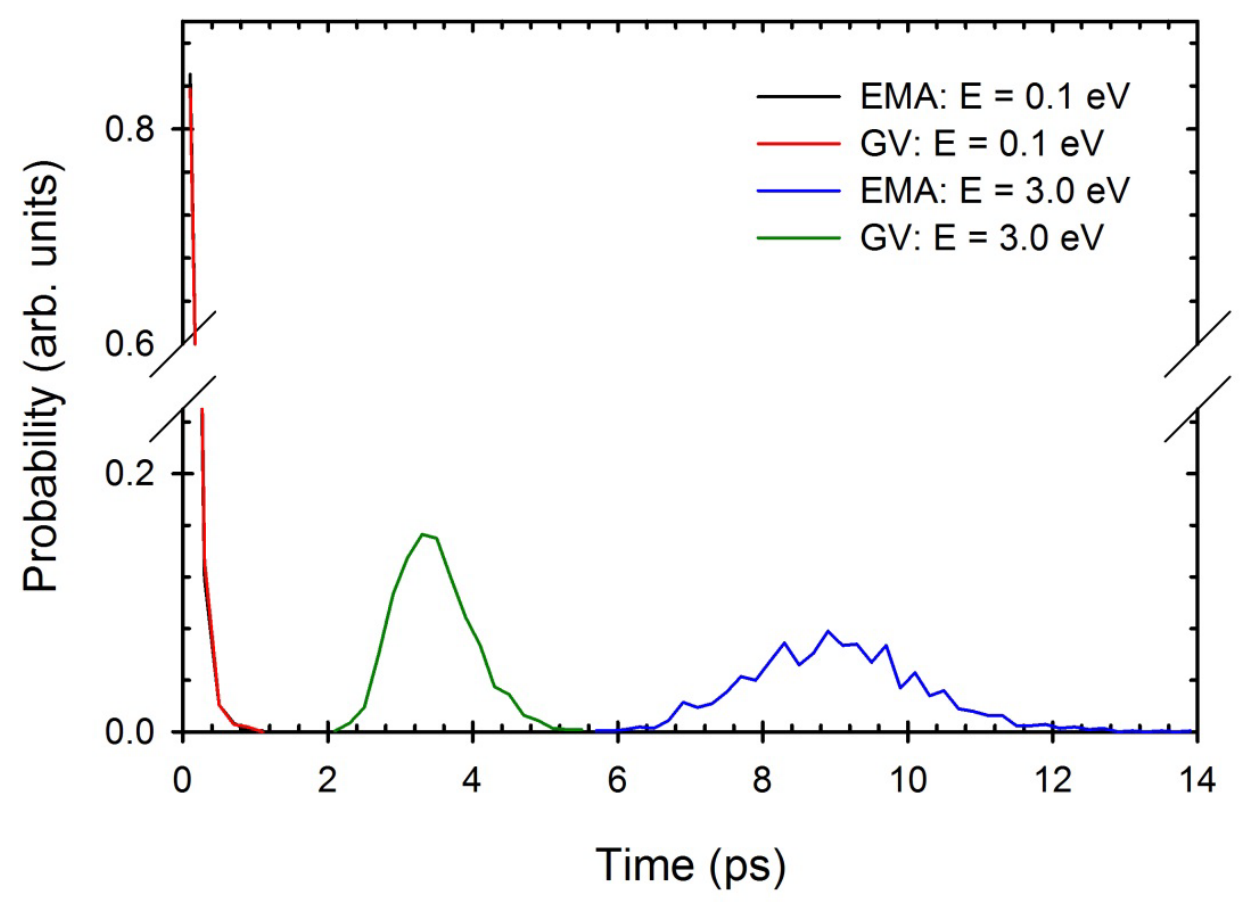




\section{Thermalization distances}

Recombined particles: $2 \mathrm{keV}$ incident y-ray
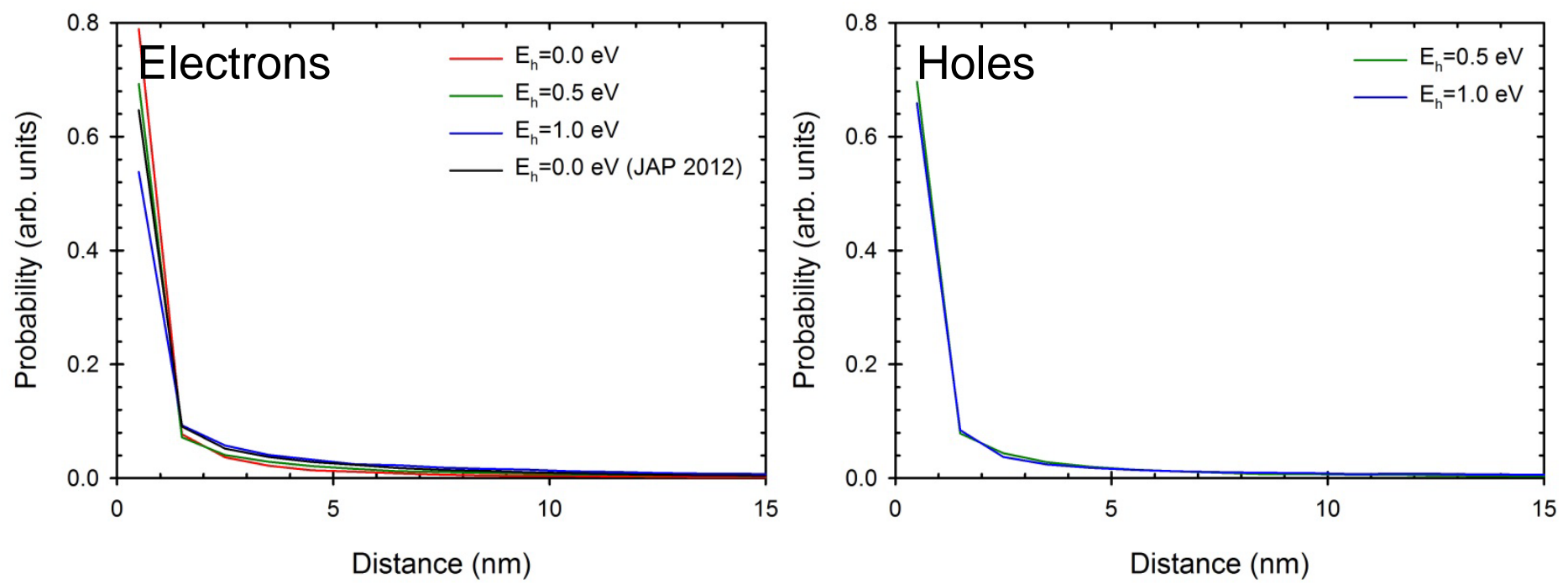

Stopped particles: $2 \mathrm{keV}$ incident $\mathrm{y}$-ray
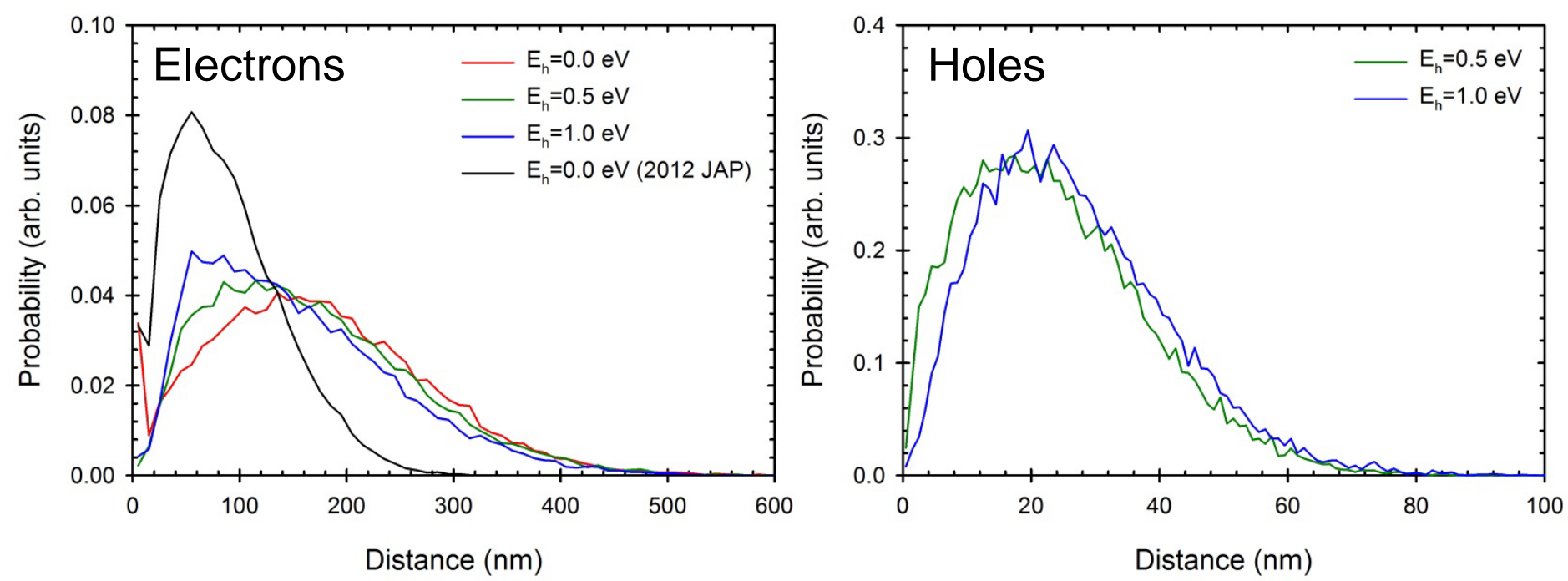


\section{Thermalization times}

Stopped particles: $2 \mathrm{keV}$ incident $\mathrm{y}$-ray
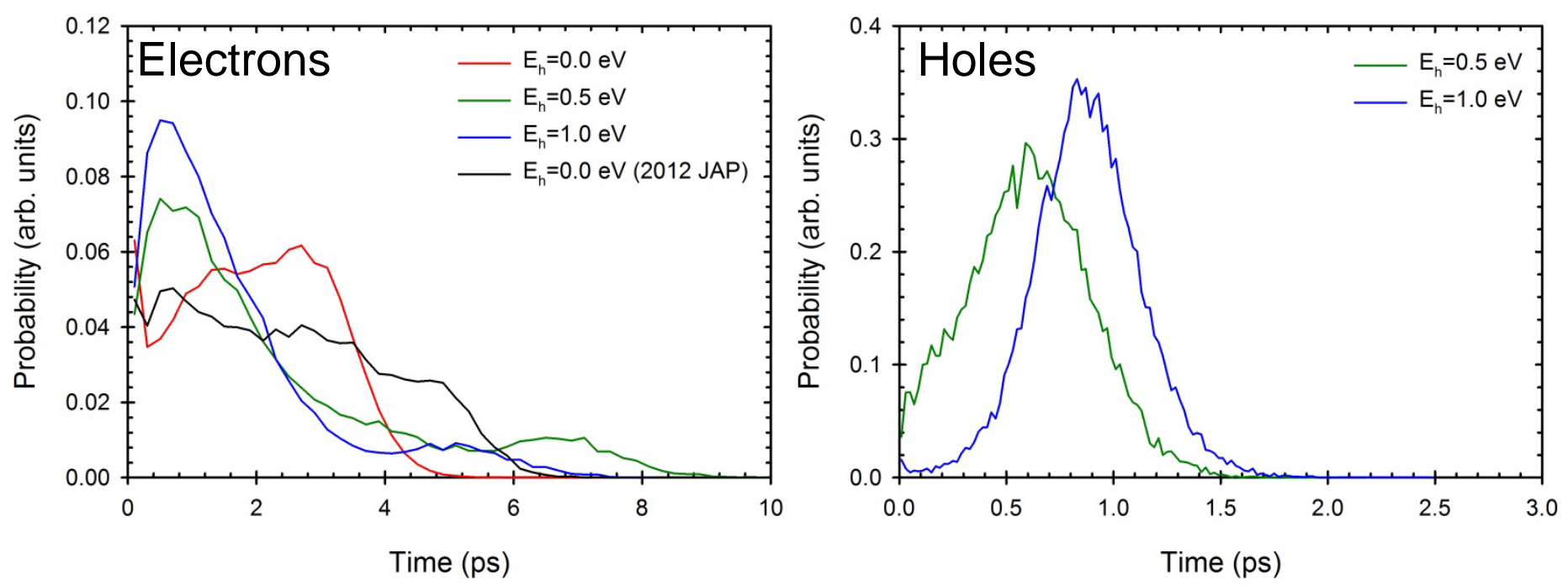

Stopped particles: $E_{h}=0.75 \mathrm{eV}$
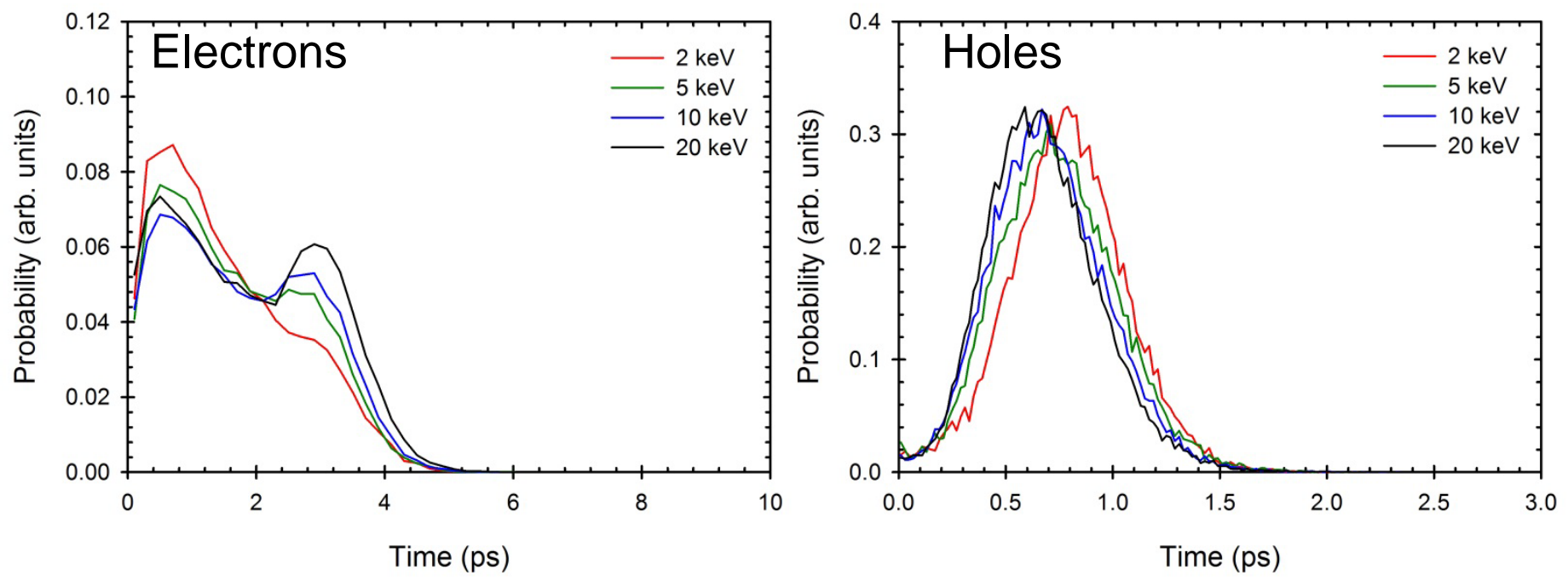


\section{Electron-hole pair recombination}

Increasing incident energy...

...decreasing proportion of high-density regions ...decreasing extent of recombination
Increasing hole energy...

...increasing probability to encounter electron

....increasing dilution of e-h pair track

...variable extent of recombination

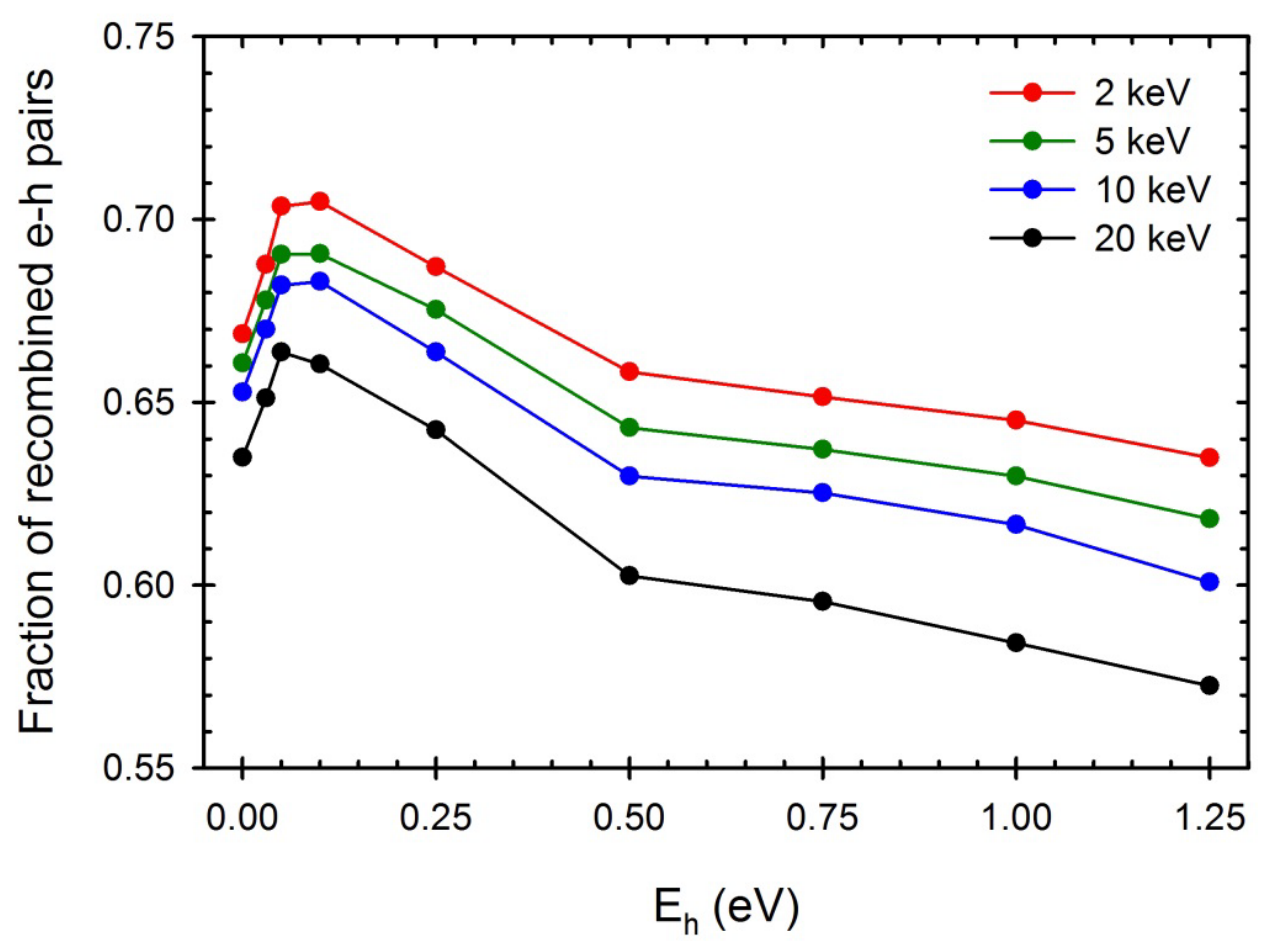


- Group velocity model yields better agreement with exp. data than effective mass approximation

- Electron-hole pair recombination is fast and can be extensive

- Electron-hole pair recombination is dependent on incident energy and initial hole energies

- Thermalization leads to considerable charge separation

- Hole thermalization is faster than electron thermalization $\rightarrow$ implications for nonlinear quenching 


\section{Kinetic Monte Carlo (KMC) model of scintillation processes}

- Atomic-level simulation of the kinetics and efficiency of scintillation processes

- A wide range of mechanisms can be simulated

Pre-exponential factor, $\mathrm{A}$, and activation energy, W, needed to parameterize each process.

$$
k=A \times \exp \left(-W / k_{\mathrm{B}} T\right)
$$

- KMC simulations allow for explicit representation of inherent heterogeneous nature of ionization tracks

- Nonlinear quenching (in collaboration with WFU)

Use data measured under simpler conditions than a $y$ ray track to isolate specific properties

Z-scan/photon response data for UV-excited $\mathrm{Nal}(\mathrm{TI})$ and

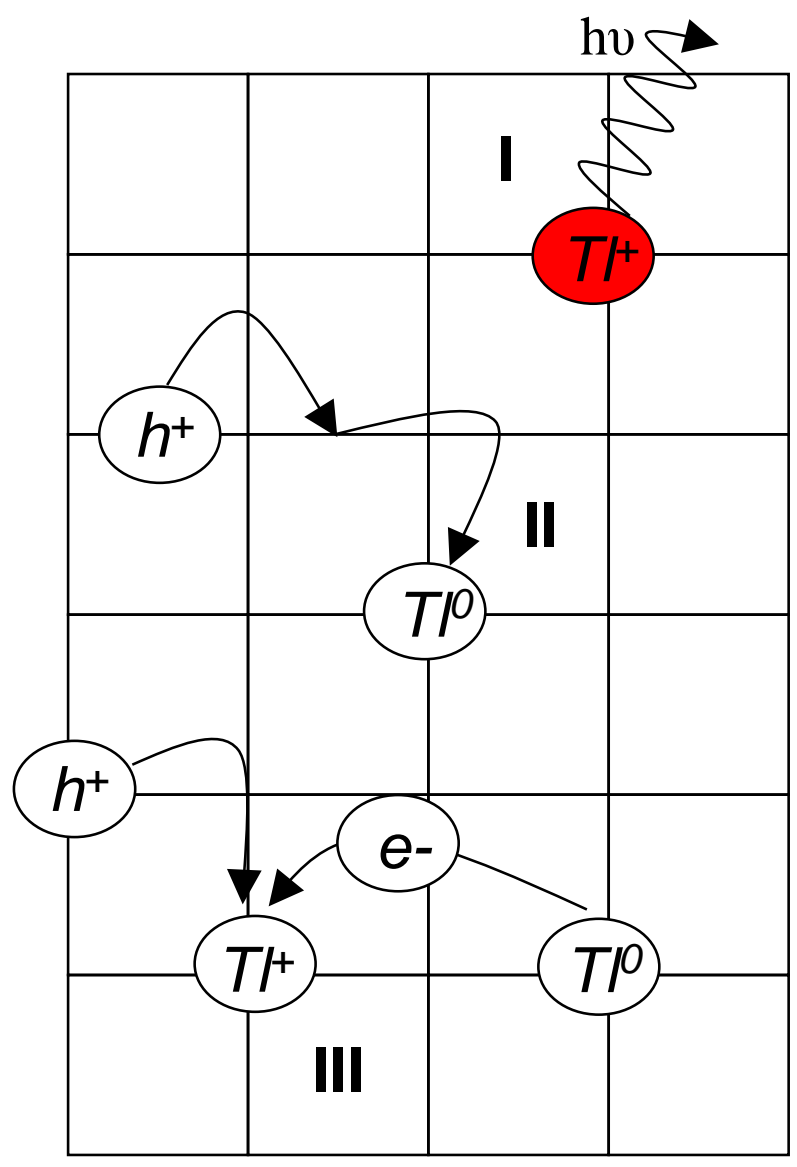

$\mathrm{CsI}(\mathrm{TI})(5.9 \mathrm{eV})$ obtained by Williams and Grim at WFU 


\section{Model parameters}

\section{From first-principles calculations...}

STH/STE diffusion

\section{From experiment...}

STE radiative decay

STE non-radiative decay
Decay rate of pure Nal at $5 \mathrm{~K}$ - Nagata JL 1991

Kinetics of scintillation of TI-doped Nal vs. temperature - 'superfast' component - Moszyński NIM A 2006

STE effective decay (UV) Kinetics of scintillation in pure Nal - Williams PSSb 2011

$\left(\mathrm{Tl}^{+}\right)^{\star}$ radiative decay

Electron thermal release

STE dissociation (UV)

Förster transfer
Energy barriers from DFT calculations - Prange PRB 2013
Kinetics of scintillation of Tl-doped Nal vs. temperature - 'slow' and 'fast' components - Moszyński NIM A 2006

Time-resolved optical absorption data - Williams IEEE TNS 2010

Free parameter to reproduce data on light yield vs. excitation density

- Grim PRB 2013 


\section{Scintillation kinetics vs. temperature}

\section{Nal:0.1 mol\% TI - 662 keV y-rays}

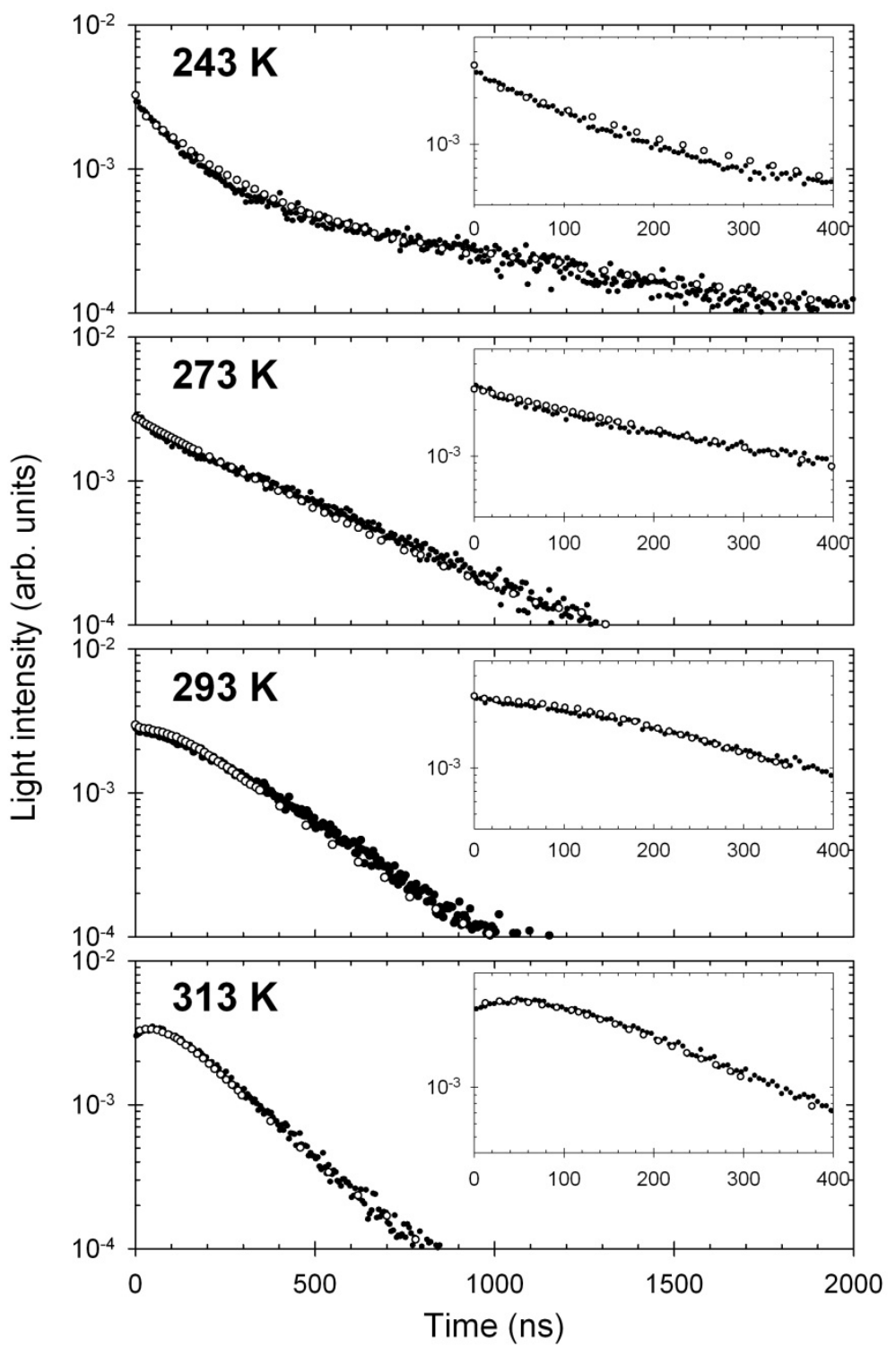

Exp. data: Moszyński NIM A 2006

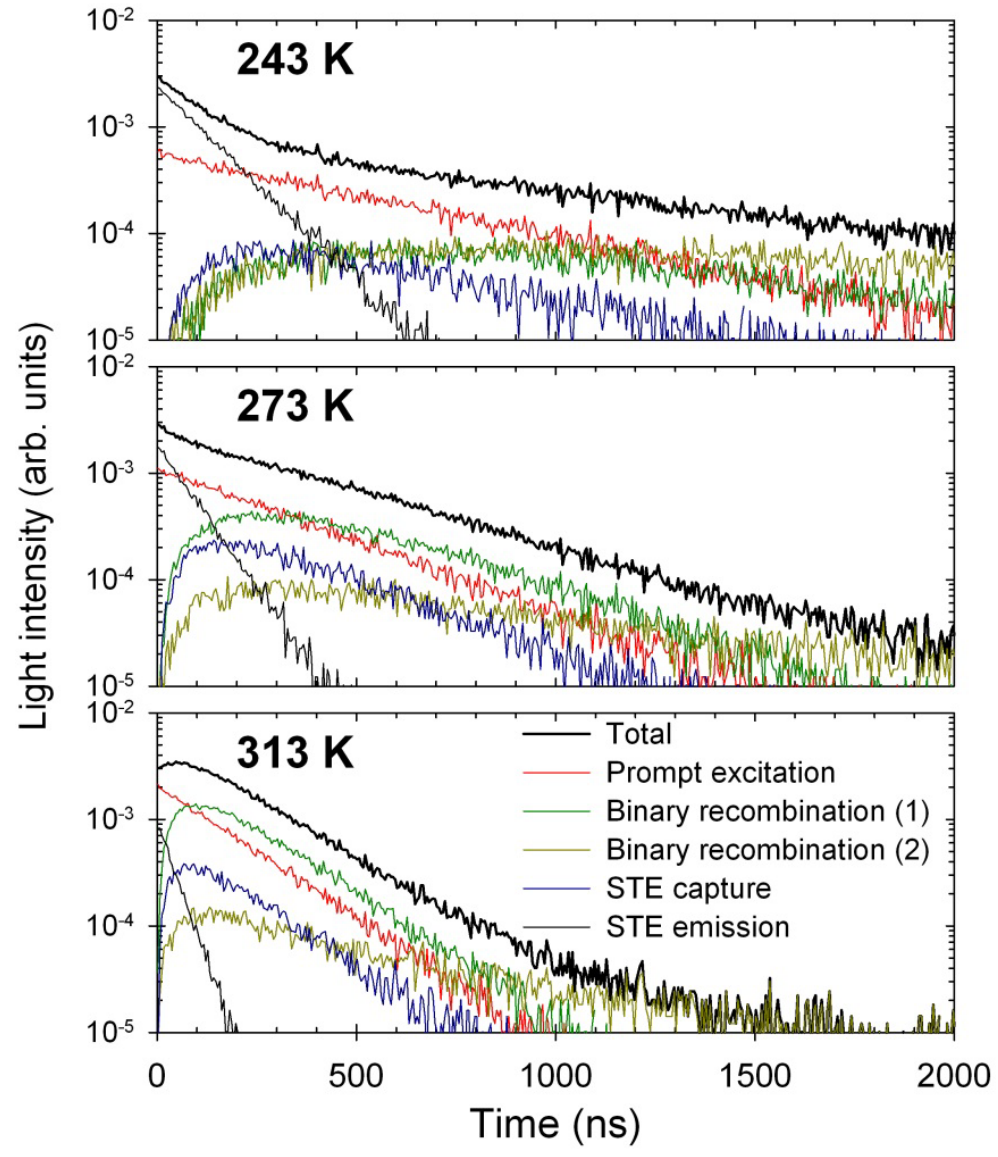

Contributions of different processes in KMC simulations help identify underlying mechanisms 


\section{Scintillation kinetics vs. TJ concentration}

TI-doped Nal - 662 keV y-rays

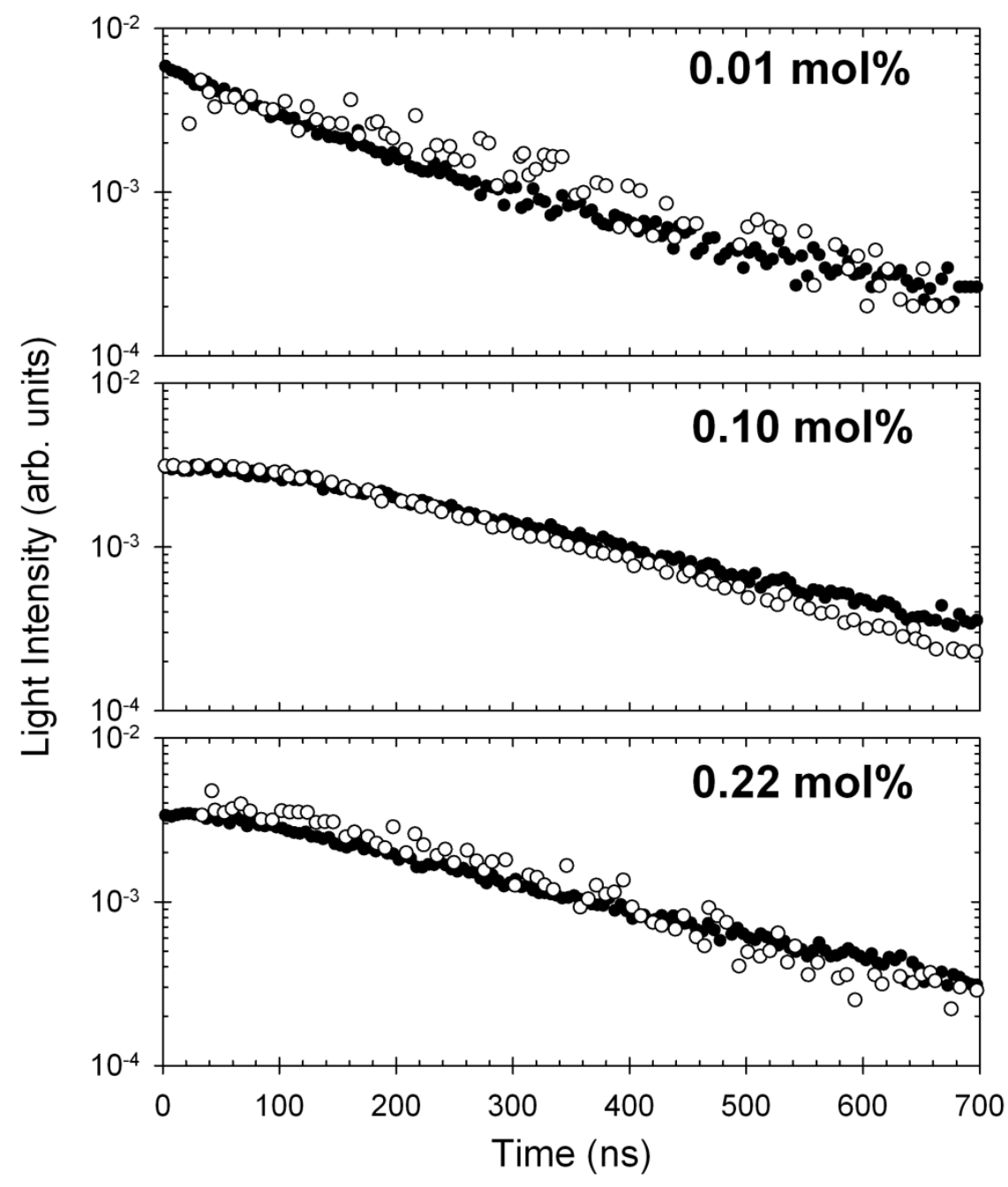

Exp. data: Kubota JPSJ 1998

KMC model also allows for simulating kinetics and efficiency of scintillation at high incident $\mathrm{Y}$ ray energy as a function of $\mathrm{Tl}$ concentration.

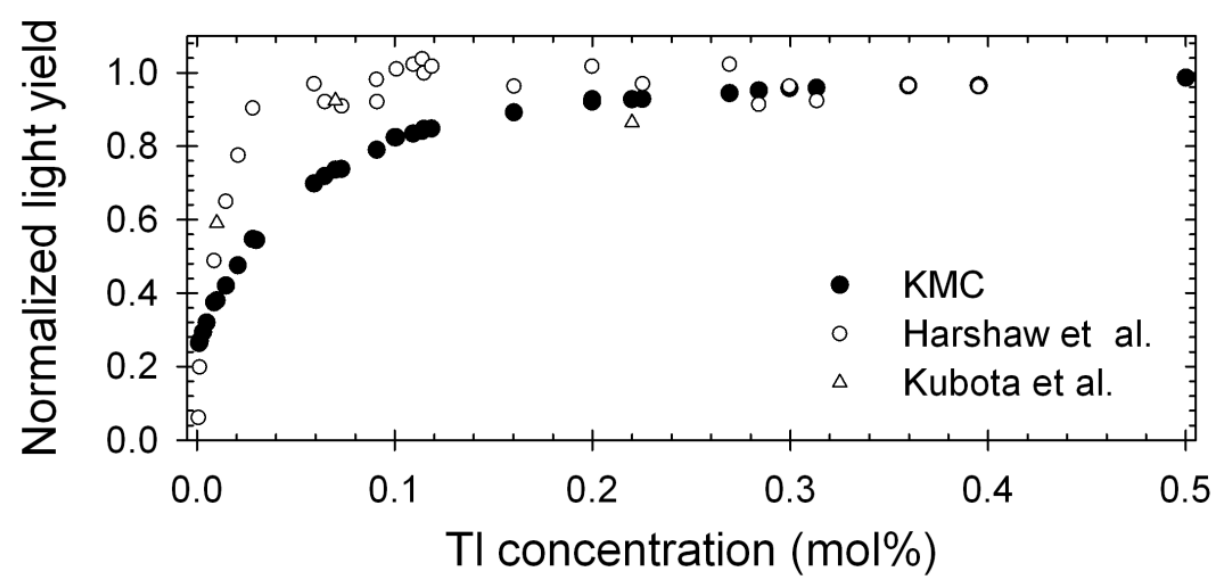

- Increase the proportion of 'stopped' electrons leads to decrease in yield with decreasing $\mathrm{TI}$ concentration. 


\section{KMC modeling of z-scan experiments}

\section{Simulation setup}

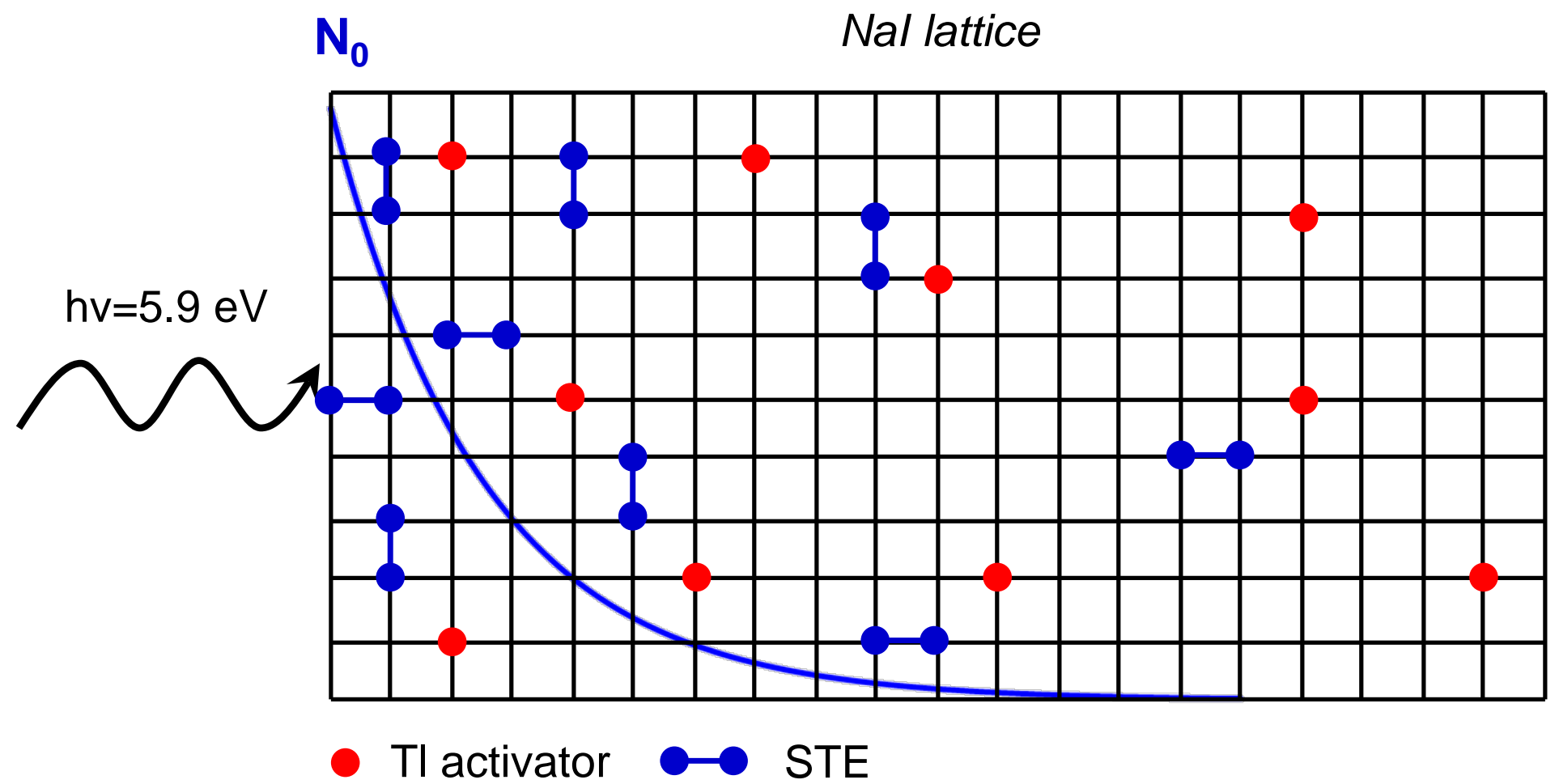

-Typical simulation cell: $32 \times 32 \times 256 / 512=\sim 10-15 \times 10-15 \times 120-170 \mathrm{~nm}^{3}$

- PBC in $x$ and $y$ directions

- $\mathrm{N}_{0}$ calculated from on-axis laser fluence, absorption coefficient, excitation energy, and position of beam waist used in the z-scan experiments 


\section{STE-STE annihilation}

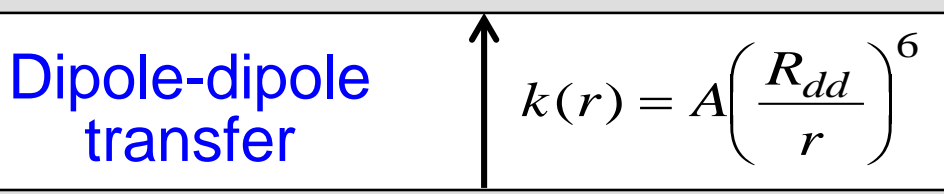

\section{STE luminescence}

\section{Self-trapped Exciton (STE)}

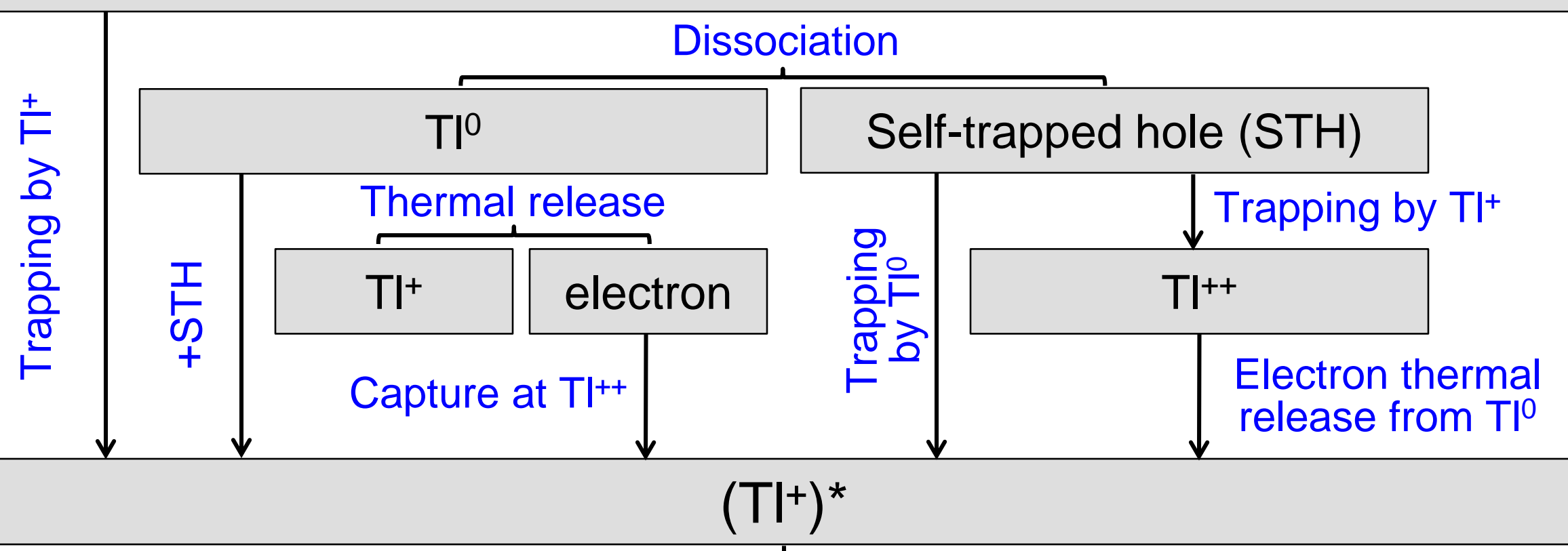

Radiative decay

Thallium luminescence 


\section{Scintillation efficiency vs. excitation density}

Nal:0.1 mol\% TI - 5.9 eV excitation Exp. data: Grim PRB 2013

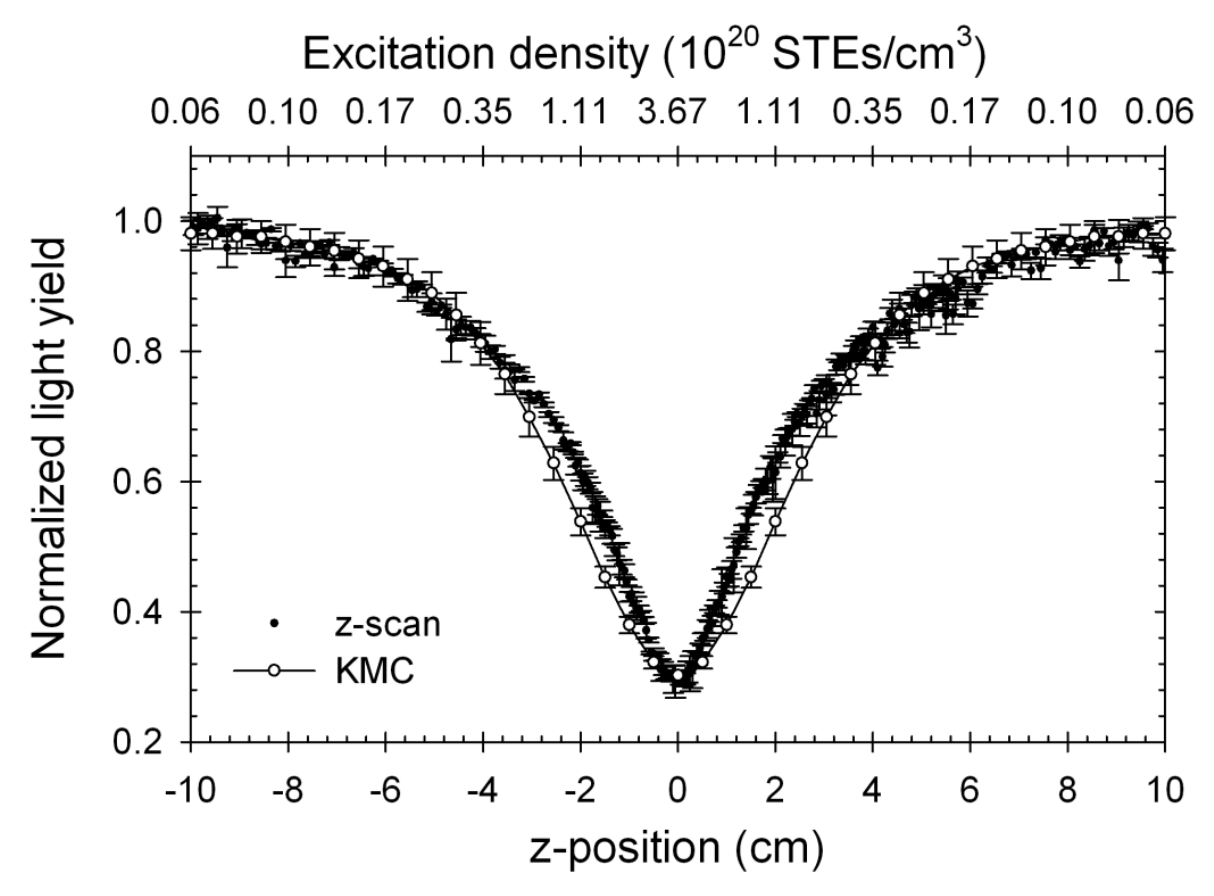

Time evolution of species populations

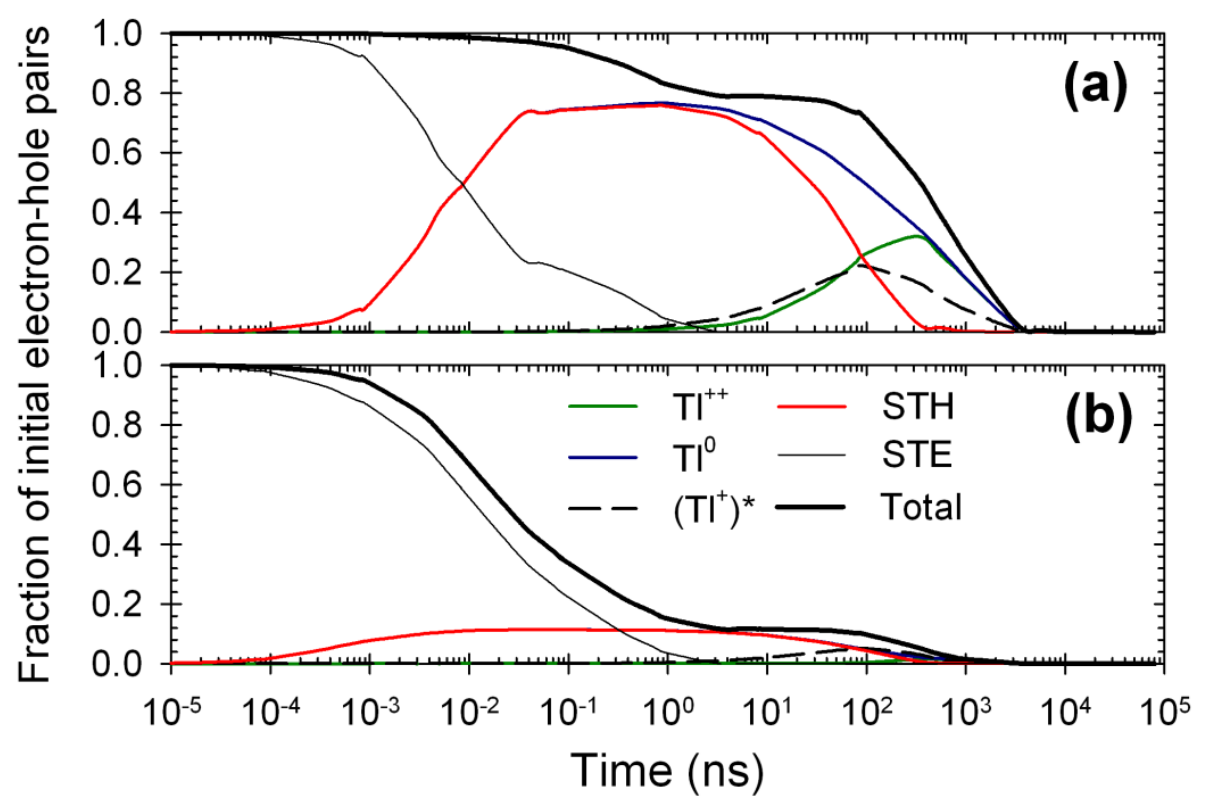

\section{$R_{\text {dd }}$ value $=2.91 \mathrm{~nm}$}

Grim et al. (analytical) $=2.9 \mathrm{~nm}$

- Small differences in light yield likely due to the assumption of flat transverse profile

- Simulations allow for determining time evolution of relevant species $\left(\mathrm{STE}, \mathrm{STH}, \mathrm{Tl}^{0},\left(\mathrm{TI}^{+}\right)^{*}, \ldots\right)$. 
Kerisit et al. IEEE TNS 61 860-869 (2014)

- Kinetic Monte Carlo model was developed to simulate the scintillation response of $\mathrm{Nal}(\mathrm{Tl})$ as a function of temperature, $\mathrm{Tl}$ concentration, and excitation density

- New KMC parameter: Förster transfer radius for describing interactions between excitations. Yields good agreement with photon response data (WFU)

- Will enable improved simulations of nonproportionality once combined with realistic ionization track structures calculated by NWEGRIM 


\section{Science-Driven Candidate Search for New Scintillator Materials}

\section{Sebastien Kerisit, Yulong Xie, Luke W. Campbell, Micah P. Prange, and Dangxin Wu}

Pacific Northwest National Laboratory

\section{Fei Gao}

University of Michigan

This project is funded by the National Nuclear Security Administration, Office of Defense Nuclear Nonproliferation, Office of Nonproliferation and Validation Research \& Development (DNN R\&D/NA-22), U.S. Department of Energy.

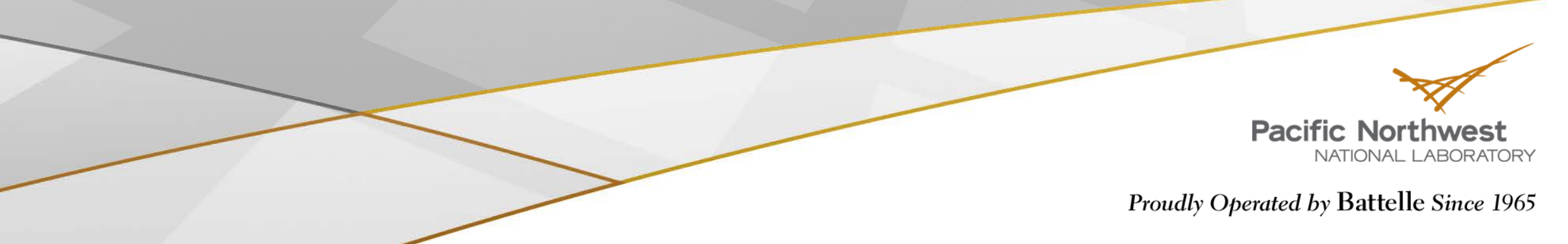


- Motivation and objective

- General approach

- Scientific team

- Project structure

- Progress to date and on-going activities

- Impact

- Plan for FY15

- Vision for future 


\section{Motivation \& Objective}

\section{Motivation}

- Why some scintillator materials offer better performance than others? (e.g. nonproportionality)

-What are the fundamental performance limits of a given scintillator material?

- Fundamental physical processes that control scintillator performance are not well understood.

- Need for developing comprehensive scintillator physics models/computer codes.

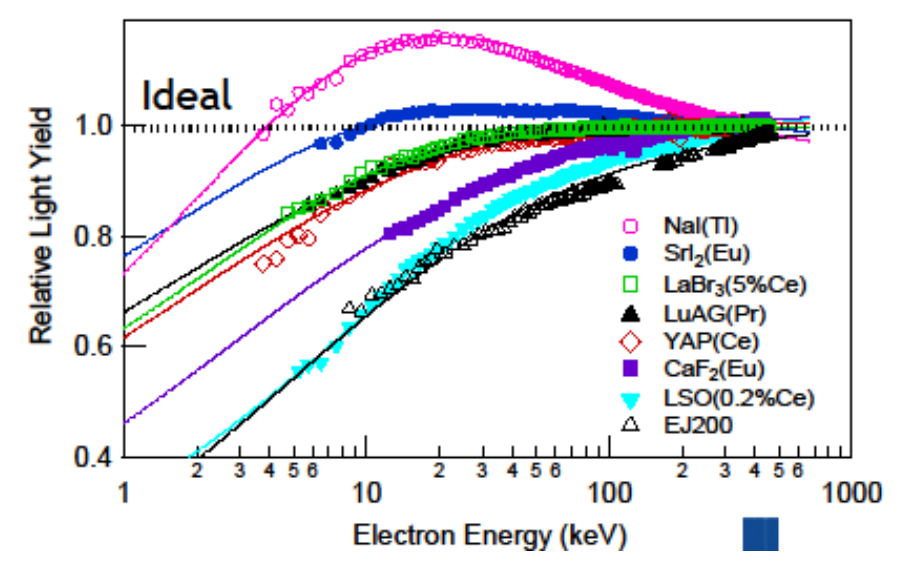

S. Payne - SPIE meeting 2011

\section{Objective}

\section{Develop predictive models to understand radiation response}

Thereby providing a theoretical basis to:

- Improve the performance of current scintillators

- Accelerate the candidate search for new scintillator materials 


\section{Overview of Approach}

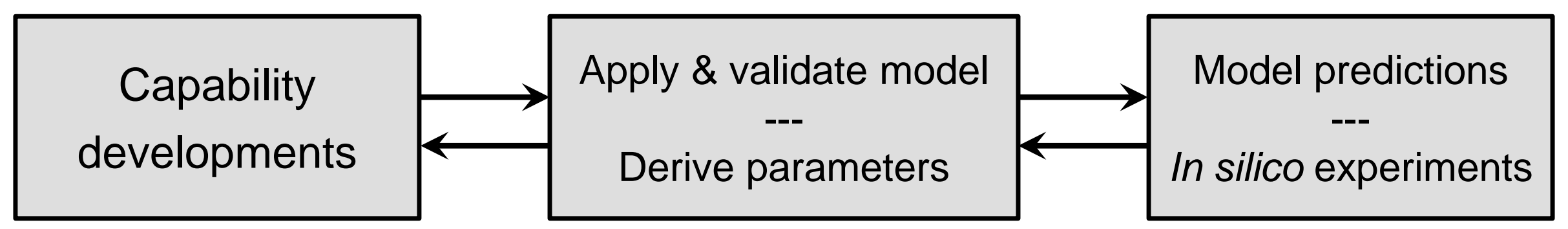

\section{Capability developments}

- Electronic ReSPonse (ERSP)

Low-energy cross sections and plasmon decay spectra

- NorthWest Electron \& Gamma-Ray Interaction with Matter (NWEGRIM)

Energy cascade $\rightarrow$ generation of electron-hole pairs

- Deformable Density (DefDen) model

Electron/hole-phonon interactions

- Kinetic Monte Carlo (KMC) code

Carrier thermalization, transport, recombination, and luminescence

\section{Model applications and predictions}

- Wide range of scintillators (alkali/alkaline-earth/lanthanum halides, oxides, elpasolites)

- Light yields, decay kinetics, nonproportionality, pulse shape discrimination

- Establish rules that dictate scintillator performance (self-consistent analysis)

- Material engineering (co-doping, defect manipulation) 


\section{Scientific team}

\section{Integrated project team with a wide range of fields of expertise...}

- Sebastien Kerisit

Computational solid-state chemistry

KMC developer, scintillation mechanisms

- Fei Gao (now at University of Michigan)

Computational materials science

NWEGRIM developer, radiation interaction/damage modeling

- Yulong Xie

Statistical analysis, data mining and optimization

NWEGRIM developer, data manipulation

- Luke W. Campbell

Computational solid-state physics

ERSP developer, theory of electronic excitations

- Micah P. Prange - postdoctoral associate (10-2011)

Computational solid-state physics

DefDen developer, theory of vibrational excitations

- Dangxin Wu - postdoctoral associate (05-2013)

Computational materials science

Electronic structure and Monte Carlo simulations 


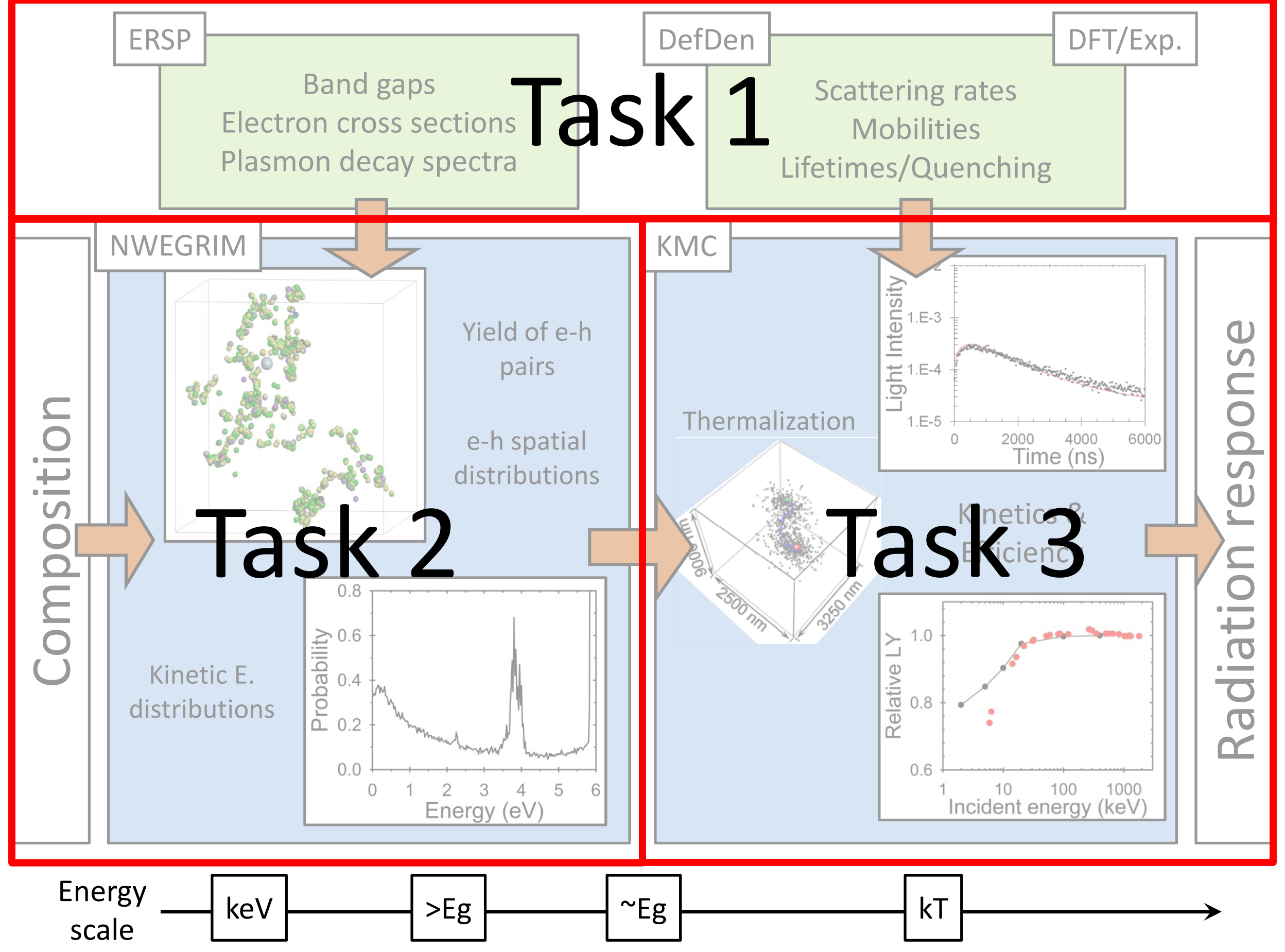




\section{Project structure}

- Task 1: Ab initio calculations of electronic properties, electronic response functions and secondary particle spectra

\section{Luke W. Campbell and Micah P. Prange + collaboration with LLNL}

Provide ab initio-based inputs to Monte Carlo models of energy cascade, thermalization, and scintillation processes. ERSP code will provide cross sections and secondary spectra of electrons and holes in low-energy region. Ab initio calculations of information carrier transport will inform kinetic Monte Carlo models of scintillation.

- Task 2: Intrinsic response properties, theoretical light yield, and microscopic description of ionization tracks

\section{Fei Gao, Yulong Xie, and Dangxin Wu}

Develop and extend NWEGRIM and its algorithms to simulate complete series of scintillator materials and evaluate their intrinsic properties, stopping power and its fluctuations, maximum theoretical light yields and track structures.

- Task 3: Kinetics and efficiency of scintillation: nonlinearity, intrinsic energy resolution, and pulse shape discrimination

\section{Sebastien Kerisit and Dangxin Wu + collaboration with WFU}

Develop and extend kinetic Monte Carlo models of thermalization and scintillation mechanisms with the specific aims to evaluate the rates of thermalization, quantify the kinetics and efficiency of scintillation, and model nonproportionality and pulse shape discrimination. 


\section{Task 1}

\section{Electronic response functions - ab initio data model}

- Sum over possible excitations in the material to determine

- Polarizability

- Rate of creation of excitations

- Interaction cross sections, power loss, and mean free path of low-energy electrons

- Fit low-energy cross section of NWEGRIM

- Spectra of particles created by electron

- Provide spectrum of secondary particles from plasmon decay for NWEGRIM

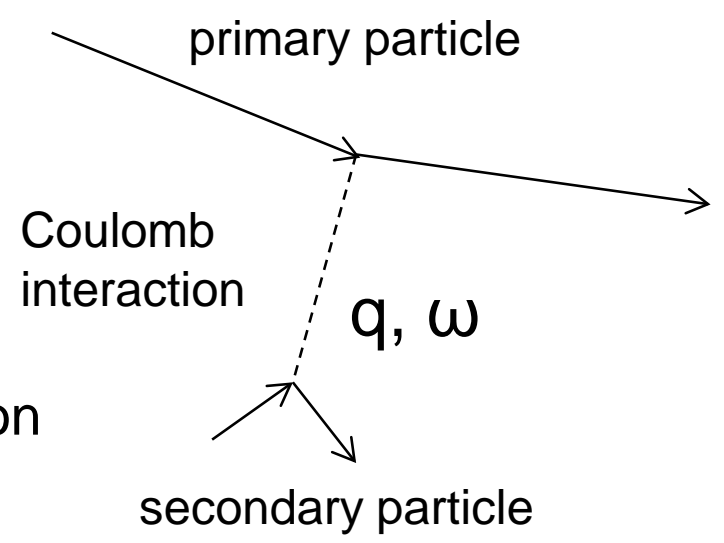

\section{Example: $\mathrm{BaF}_{2}$}

Electron cross sections

Low-energy behavior is critical to capturing fluctuations near the track end
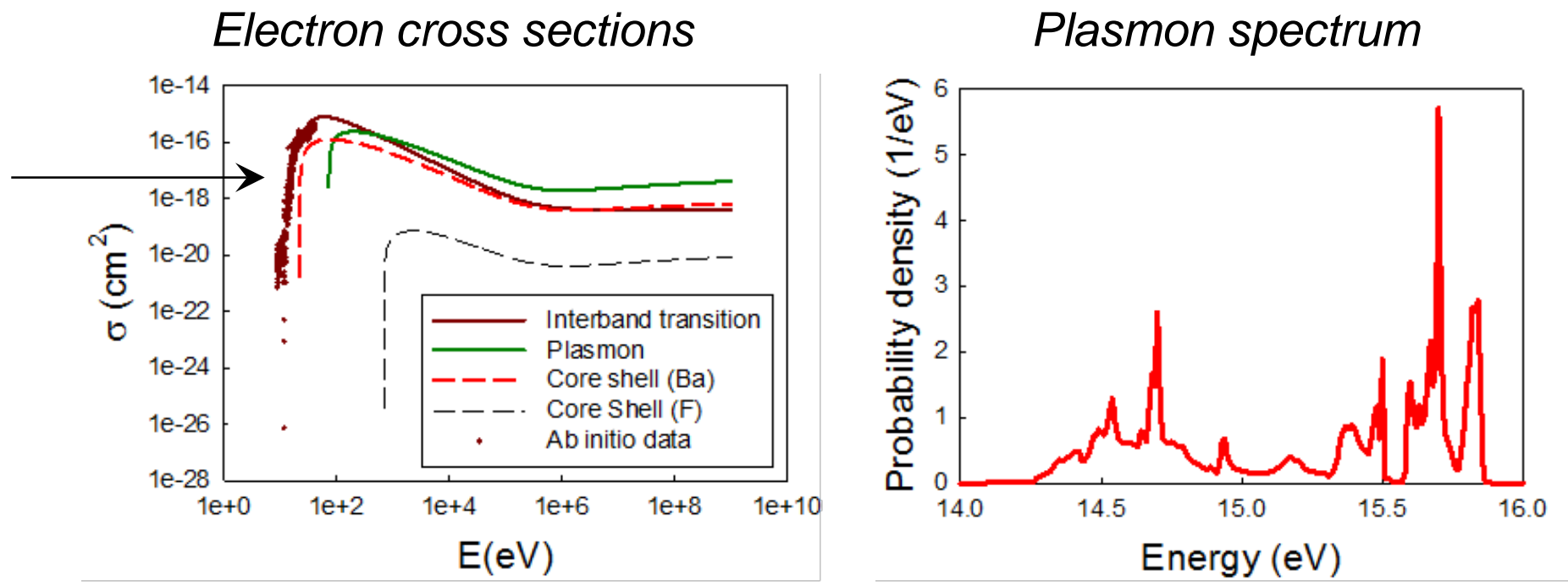


\section{Task 1}

\section{Electronic losses of heavy particles}

- Significant interest in materials that scintillate under neutron radiation

- Fast neutrons detected by nuclear recoil from collisions with neutrons (esp. protons)

- Slow neutrons detected by absorption interactions

$$
\begin{aligned}
& \text { - } \mathrm{n}+{ }^{6} \mathrm{Li} \rightarrow \alpha+\mathrm{T} \\
& \text { - } \mathrm{n}+{ }^{10} \mathrm{~B} \rightarrow \alpha+{ }^{7} \mathrm{Li}
\end{aligned}
$$

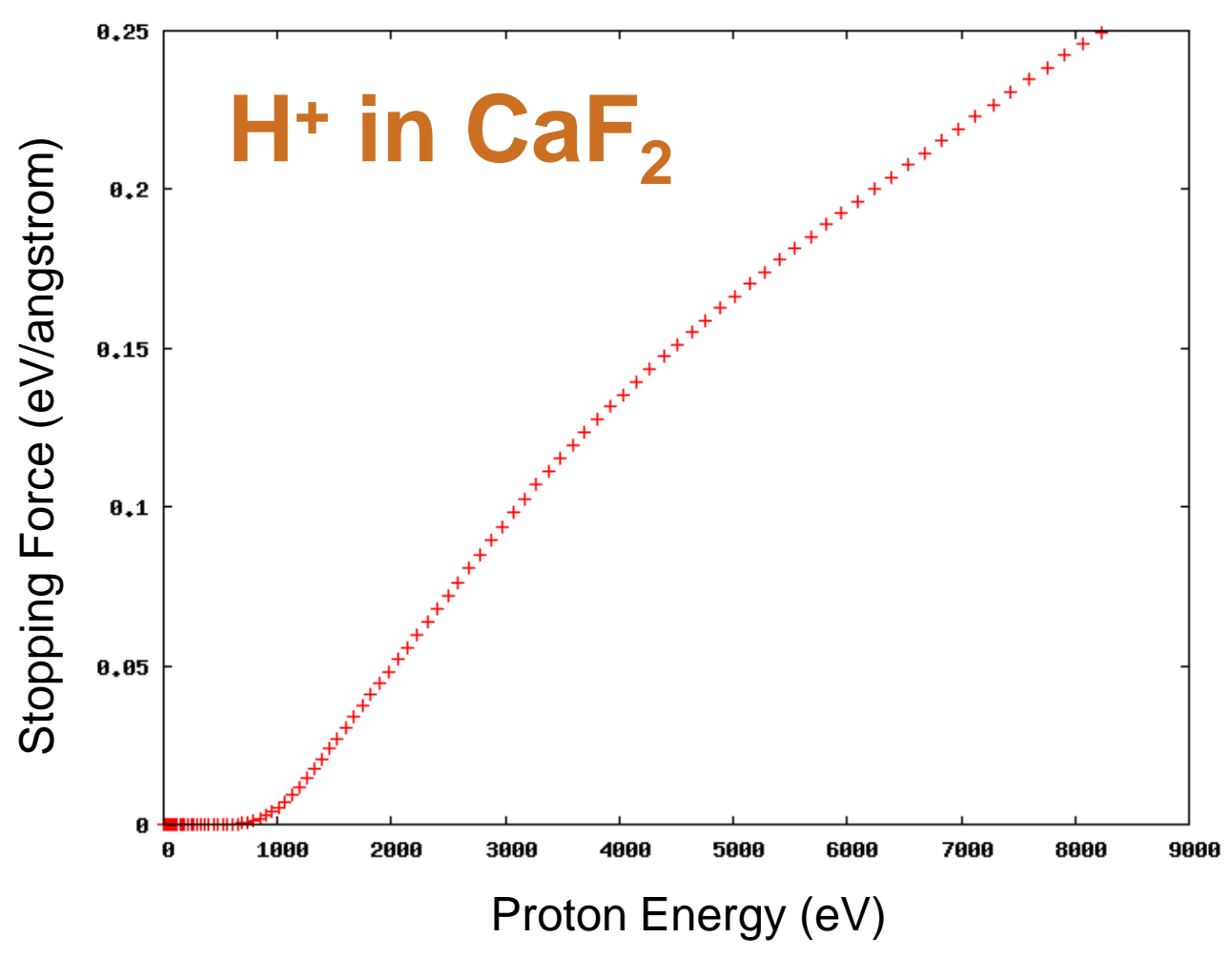

- Need to be able to predict scintillation behavior of energetic nuclei

- Preliminary code completed for heavy ion response in ERSP

- Predict interaction rates and power loss

- First step towards simulating dual detectors (e.g. CLYC - elpasolites) 


\section{Task 1}

\section{Ab initio calculations of information carrier transport}
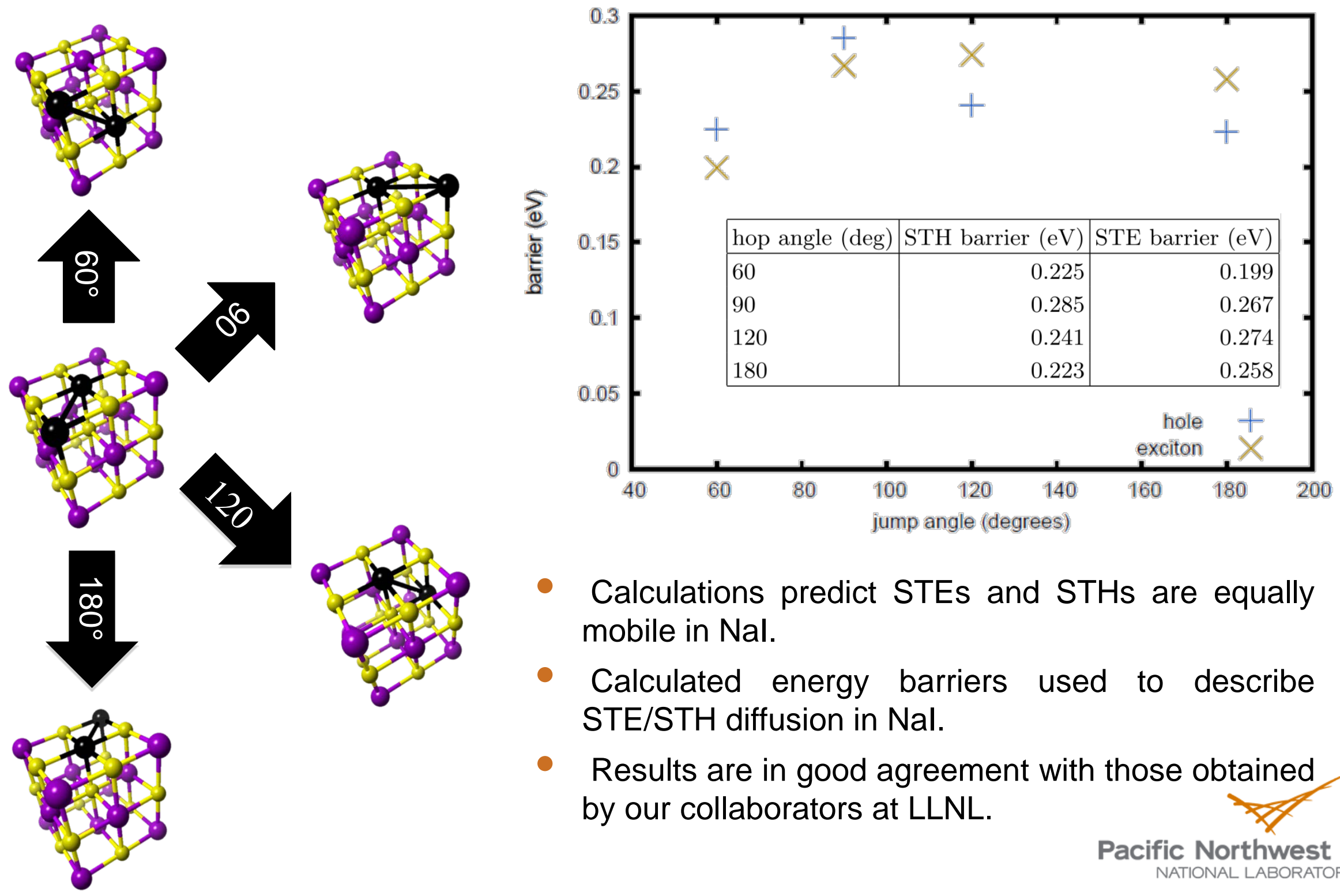

- Calculations predict STEs and STHs are equally mobile in Nal.

- Calculated energy barriers used to describe STE/STH diffusion in Nal.

- Results are in good agreement with those obtained by our collaborators at LLNL. 


\section{Task 1}

\section{Ab initio calculations of vibrational excitations}

- Accurate treatment of thermalization is necessary for quantitative prediction of scintillator performance

- Direct ab initio methods: no empirical inputs or model interaction Hamiltonians

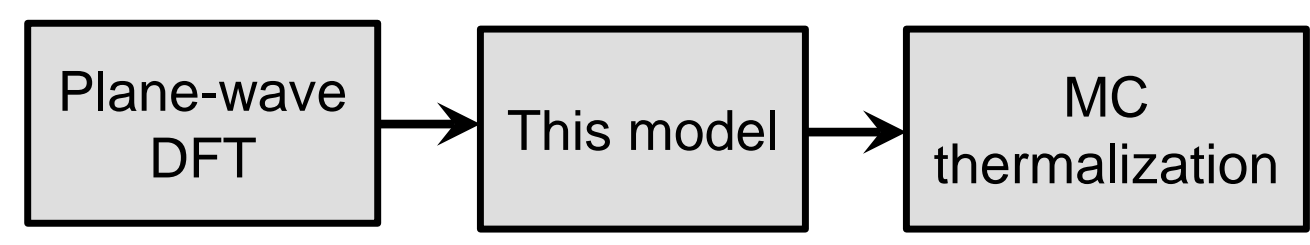

"Golden rule" for energy loss rate

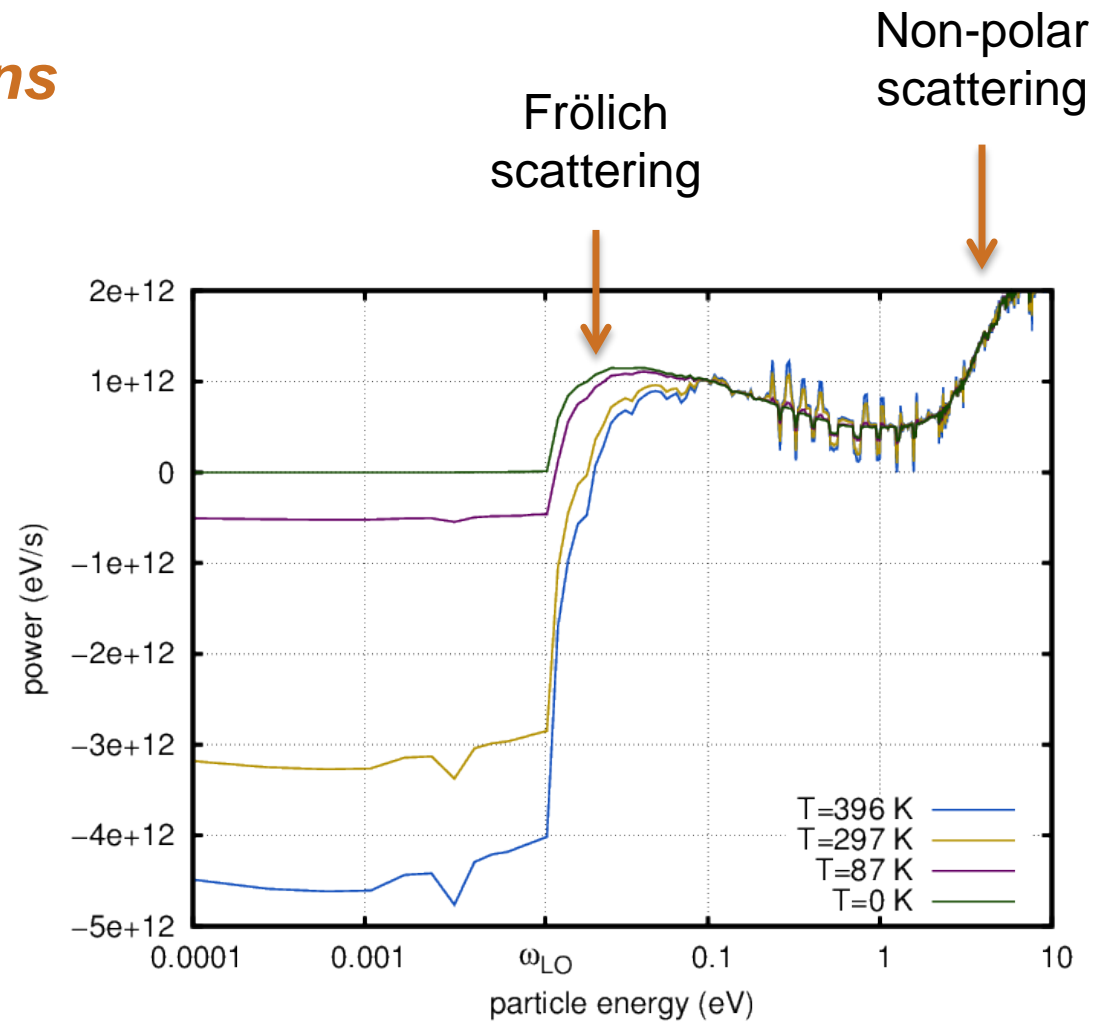

Energy loss rates in Csl

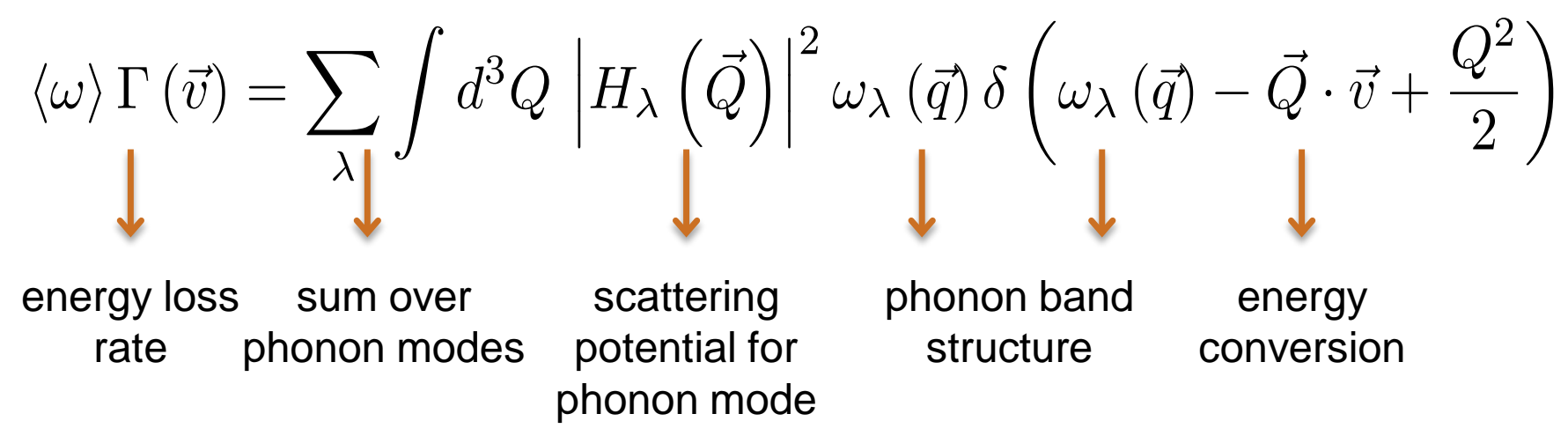

\begin{abstract}
phonon band energy structure conversion
\end{abstract}




\section{Task 2}

\section{Features}

- Low cutoff energy $\left(\sim E_{g}\right) \rightarrow$ explicit account of all ionization events

- Average energy per electron-hole pair is predicted not an input parameter

- Multiple elastic scattering theory

- Explicit calculations of density fluctuations

- Database of various electron cross sections

- Incorporate ab initio data model predictions

\section{Properties}

- Spatial distribution of e-h pairs - track structures

- W vs. particle E, max. theo. LY, and Fano factor

- Energy loss channels and stopping power

\section{Scintillator materials}

- Alkali and alkaline-earth halides (Nal, $\mathrm{Csl}, \mathrm{BaF}_{2}, \mathrm{CaF}_{2}, \mathrm{Srl}_{2}$ )

Mean energy per e-h pair

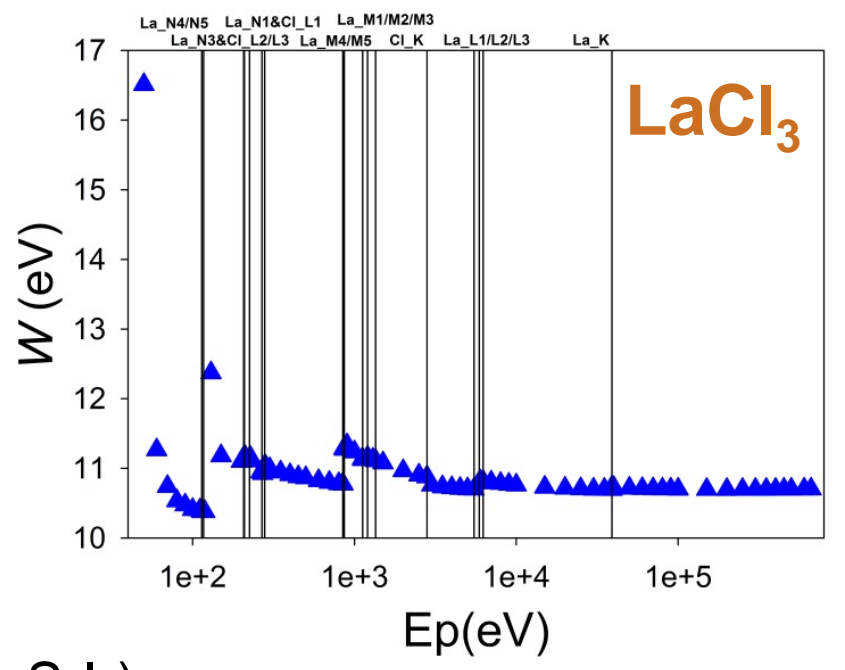

- Lanthanum halide series $\left(\mathrm{LaF}_{3}, \mathrm{LaCl}_{3}, \mathrm{LaBr}_{3}, \mathrm{Lal}_{3}\right)$

- Oxides (YAG, YAP)

- Elpasolites (CLLC, CLLC, CLYB, CLYC) 


\section{Task 2}

Maximum theoretical light yields

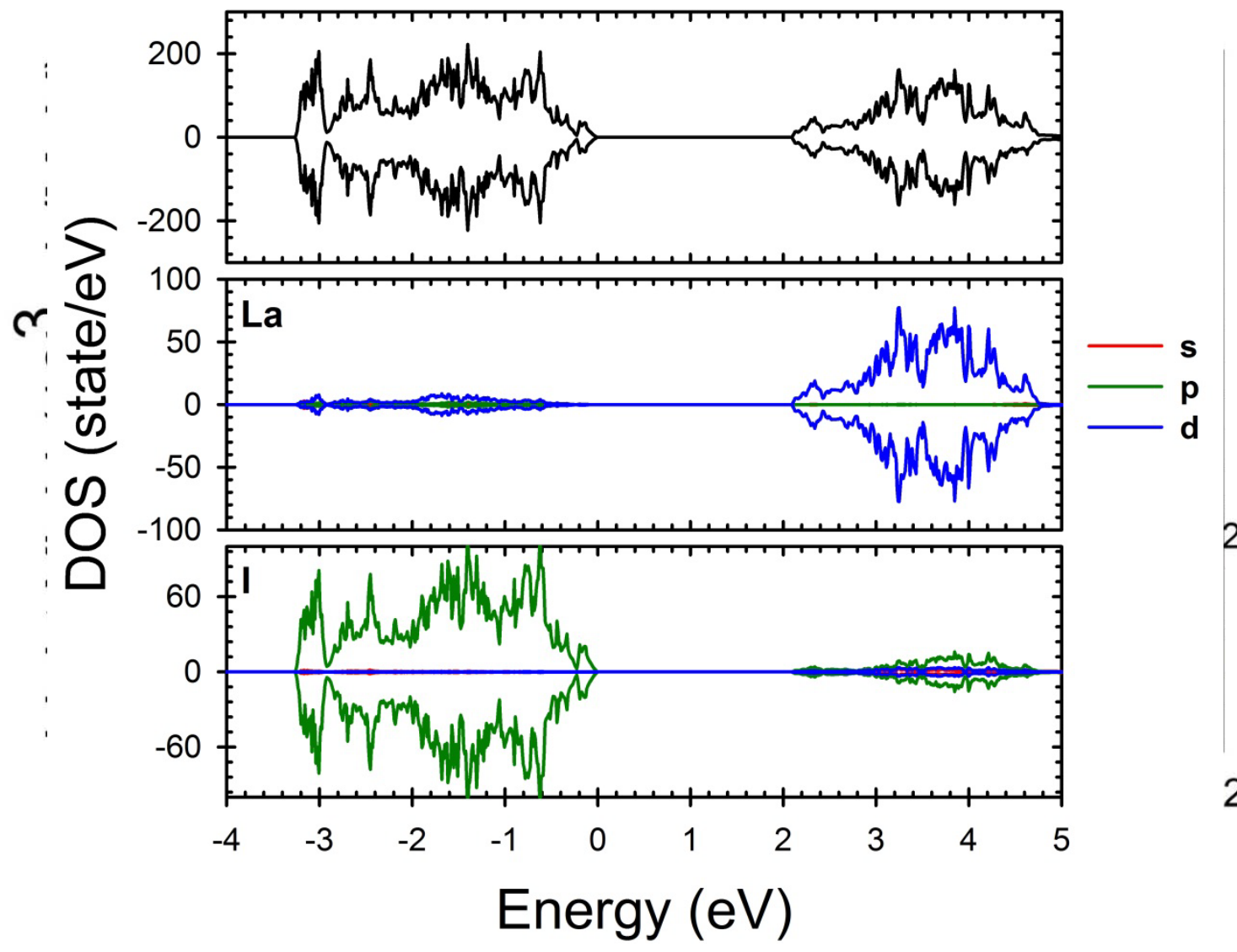

NWEGRIM can be used for rapid evaluation of:

- the potential brightness of a candidate scintillator

- the potential for improvement of current scintillators

- the upper limit for absolute light yields 


\section{Task 2}

\section{0 keV incident y-ray}

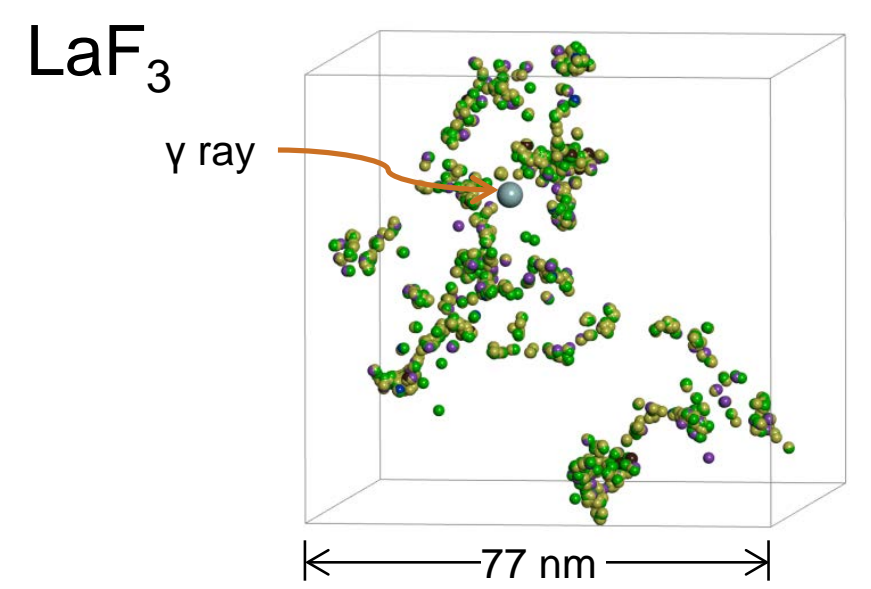

$\mathrm{LaBr}_{3}$

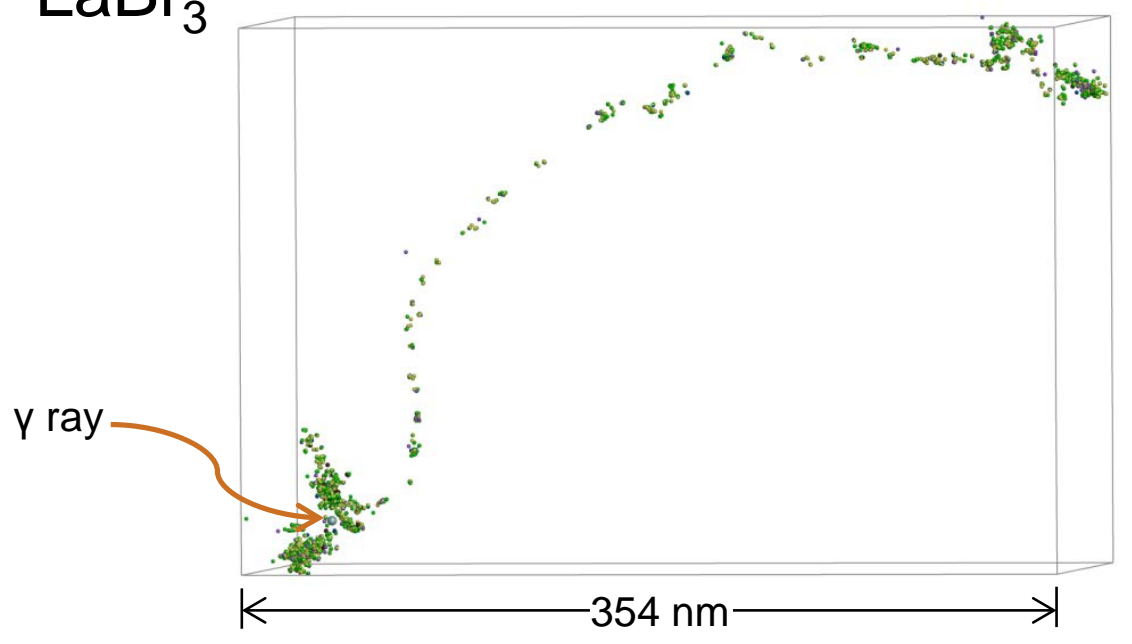

$\mathrm{LaCl}_{3}$

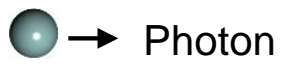

$\odot \rightarrow$ Electron (Interband)

$\odot \rightarrow$ Electron (Plasmon)

$\odot \rightarrow$ Electron (Ionization)

$\odot \rightarrow$ Electron (Relaxation)

$\odot \rightarrow$ Hole

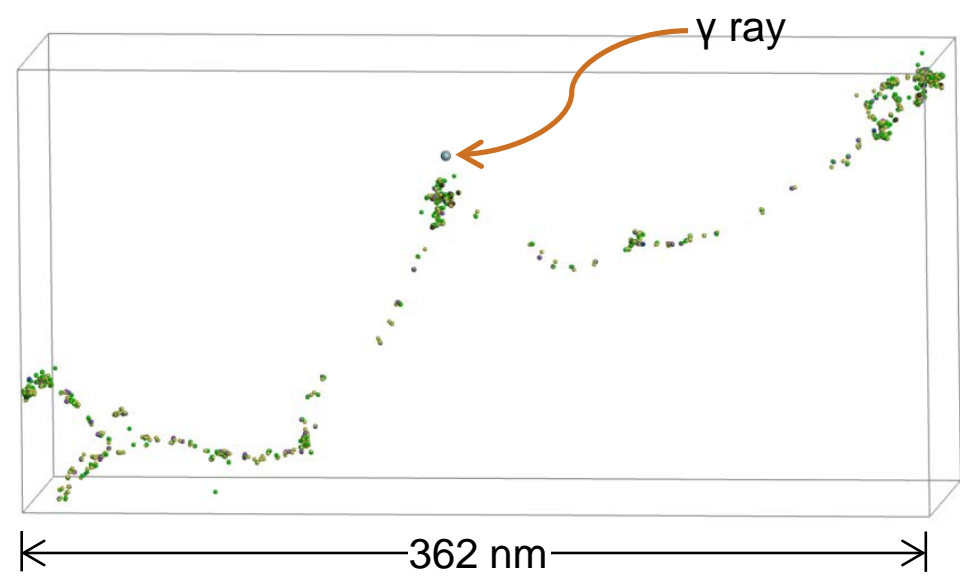

Local principle curve method

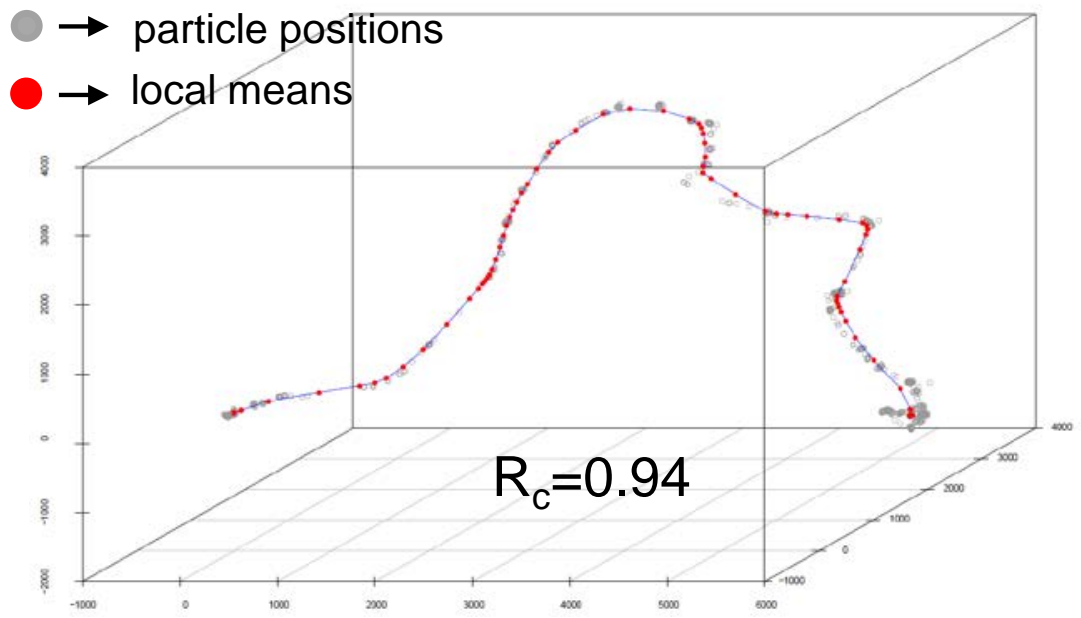

Nano-scale fluctuations in e-h pair density along the track are key to nonproportionality 


\section{Task 2}

Intrinsic properties

\begin{tabular}{|c|c|c|c|c|c|c|}
\hline Property & $\mathrm{CaF}_{2}$ & $\mathrm{BaF}_{2}$ & CsI & Nal & $\mathrm{LaBr}_{3}$ & $\mathrm{Srl}_{2}$ \\
\hline W $(e V)$ & 21.4 & 19.1 & 12.0 & 10.9 & 9.5 & 9.2 \\
\hline$\beta$ & 1.8 & 1.7 & 2.0 & 1.9 & 1.7 & 1.7 \\
\hline Interband & $63.1 \%$ & $63.7 \%$ & $65.2 \%$ & $60.2 \%$ & $72.1 \%$ & $72.2 \%$ \\
\hline Plasmon & $31.6 \%$ & $29.3 \%$ & $18.2 \%$ & $29.1 \%$ & $21.9 \%$ & $19.2 \%$ \\
\hline Ionization & $4.4 \%$ & $4.1 \%$ & $11.1 \%$ & $6.4 \%$ & $3.2 \%$ & $4.3 \%$ \\
\hline Fano factor & 0.23 & 0.19 & 0.30 & 0.28 & 0.21 & 0.23 \\
\hline $\begin{array}{c}\text { Track } \\
\text { structure }\end{array}$ & Clustered & Clustered & $\begin{array}{l}\text { Slightly } \\
\text { clustered }\end{array}$ & $\begin{array}{l}\text { Slightly } \\
\text { clustered }\end{array}$ & Linear & Linear \\
\hline
\end{tabular}

- Small number of energy loss channels (ideally, only interband transitions)

- Linear track structure that decreases nonlinear quenching at low energies 


\section{Task 3}

Kinetics and efficiency of scintillation in alkali halides

Same set of parameters

\section{Nal:0.1 mol\% TI - $662 \mathrm{keV}$ Y-rays}

Exp. data: Moszyński NIM A 2006
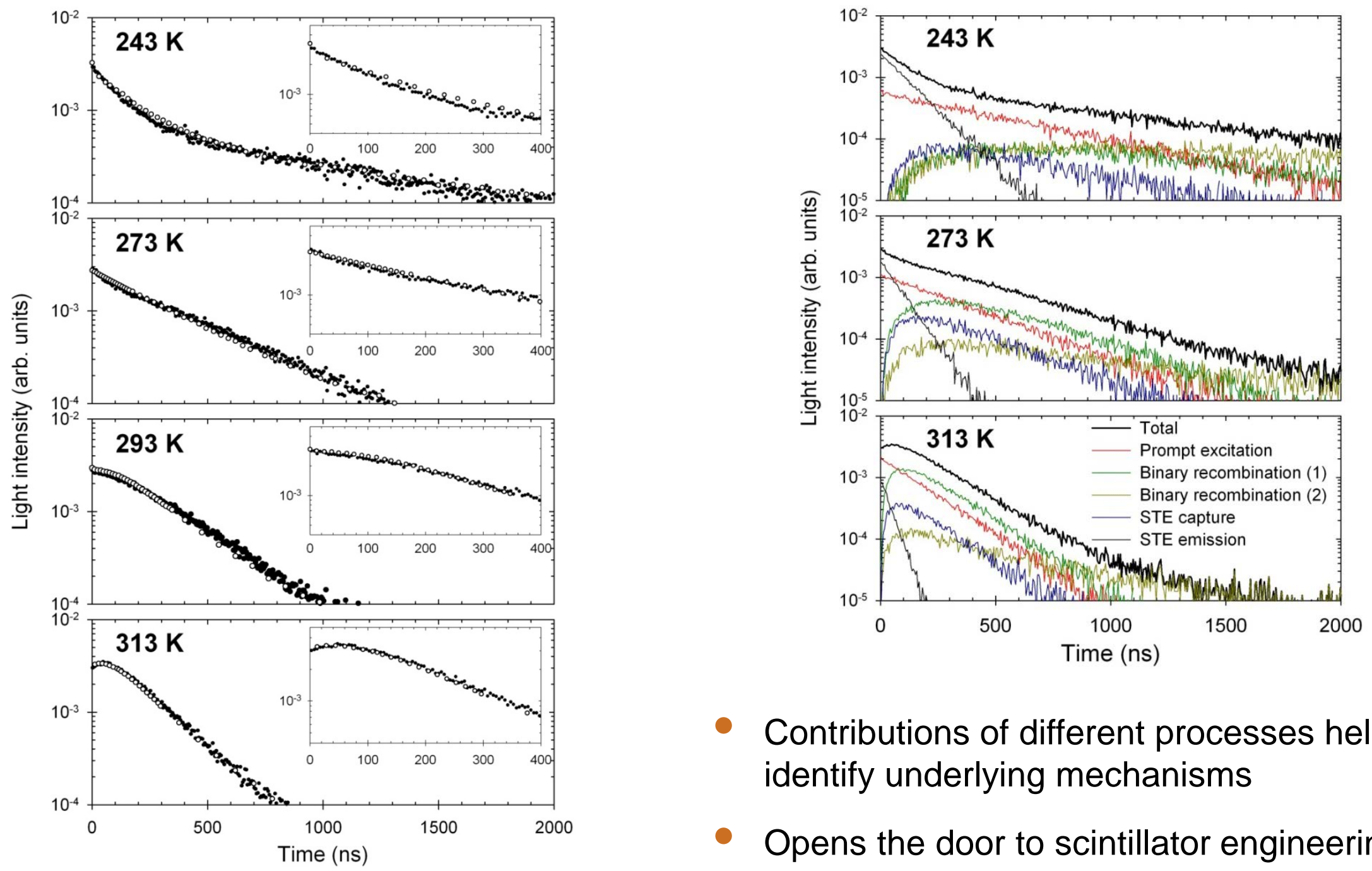

- Contributions of different processes help identify underlying mechanisms

- Opens the door to scintillator engineering 


\section{Impact: new insights}

\section{Thermalization}

Finding: Slow electron cooling affects track density, i.e. thermalization can lead to extensive charge separation.

R. Williams (WFU) worked out implications "Decision Tree" Several publications followed (e.g. A. Vasil'ev MSU)

Focus for current research (e.g. K. Biswas ASU)

\section{Ionization tracks}

Finding: Realistic ionization tracks have complex nano-scale structures and fluctuations.

Track radius at track end enters WFU's model G. Bizarri (LBNL) and S. Payne (LLNL) interested in comparing with their rate-equation and phenomenological models

\section{Scintillator physical "Decision Tree"}

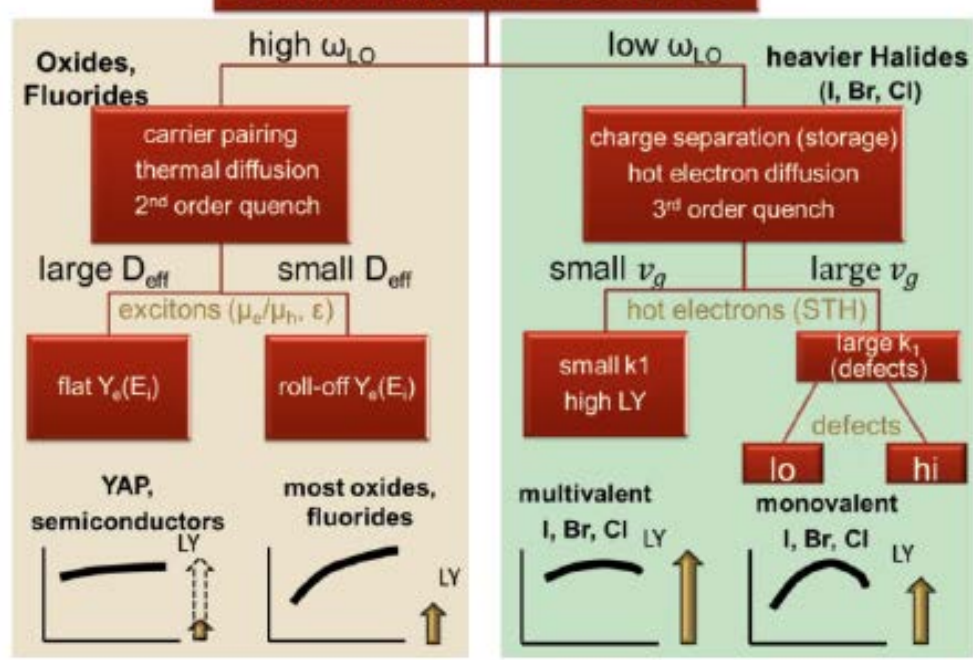

Williams et al. Proc. SPIE 8852, 88520J-22 (2013)

\section{Diffusion of self-trapped holes and excitons in alkali halides}

Finding: STEs/STHs exhibit similar energy barriers for diffusion.

Challenges long-held view of STEs being more mobile than STHs

Informed kinetic Monte Carlo models, yielded good agreement with experimental data 


\section{Main thrust: towards modeling of pulse shape discrimination}

\section{Overview}

Extend modeling capabilities to enable simulations of the response to heavy particles

Will allow for modeling key elementary processes that give rise to pulse shape discrimination

Model system: CsI(TI) (dual alpha/gamma detector - extensive experience modeling Csl)

\section{Breakdown by tasks}

\section{Task 1 (LWC)}

Develop ERSP to derive low-energy cross sections and secondary particle spectra for alphas (to be used as input in NWEGRIM).

\section{Task 2 (YX, FG, DW)}

Modify NWEGRIM to implement low- and high-energy cross sections for alphas and enable code to handle new particle type.

Generate ionization tracks for alpha particles using output from Task 1.

Subcontract to be set up to cover two summer months of FG's time at UM.

Task 3 (SK, DW, WFU and LLNL collaborations)

Complete implementation of third-order nonlinear quenching mechanism in KMC code.

Apply to Csl, Nal, and $\mathrm{Srl}_{2}$ and derive parameters from WFU's photon response data.

Apply KMC code to model nonproportionality and PSD ( $\alpha / Y)$ for CSI(TI) using output from Task 2. 


\section{Plan for FY15}

\section{Quantum-mechanical model of thermalization}

\section{Overview}

Rate and extent of nonlinear quenching strongly dependent on thermalization rates

Development of quantum-mechanical model of thermalization to be finalized in FY14

Apply to common and emerging scintillators (e.g. Csl, Nal, YAP, Srl ${ }_{2}$, etc.) in FY15

\section{Breakdown by tasks}

\section{Task 1 (MPP)}

Apply quantum-mechanical model of thermalization to a series of scintillators. Prioritize $\mathrm{Srl}_{2}$.

Task 3 (SK, DW)

Implement energy losses in KMC code and carry out simulations for a series of scintillators.

Compare against effective mass approximation and group velocity models.

\section{Suitable activator(s) for $\mathrm{Lal}_{3}$}

\section{Overview}

$\mathrm{Lal}_{3}$ identified by NWEGRIM as potentially very bright scintillator

Search for suitable activator: $\mathrm{Pr}, \mathrm{Nd}, \mathrm{Eu}, \mathrm{Tl}, \mathrm{Pb}, \mathrm{Bi}$, etc. $\rightarrow$ Example of in silico experiment

\section{Breakdown by tasks}

\section{Task 1 (DW, SK)}

$\mathrm{Ab}$ initio calculations of a series of activators in $\mathrm{Lal}_{3}$ with $\mathrm{GGA}, \mathrm{GGA}+\mathrm{U}$, and hybrid XC functionals. 


\section{Vision for future}

\section{Comprehensive scintillation physics for material engineering}

Significantly advance the understanding of scintillation mechanisms and provide pathways for engineering better scintillators by intelligent manipulation of these mechanisms

Examples:

Co-doping for temporary energy storage that can improve proportionality of response Effects on scintillation of dopants introduced to improve mechanical toughness Control properties that determine spatial/temporal distributions of electrons and holes Mitigate effects of incidental lattice defects

Collaborative effort:

PNNL, LBNL, LLNL, and WFU

Opportunities:

LBNL Venture proposal (FY15 - DNN R\&D/NA-22)

PNNL LDRD proposal on co-doping in CsI(Na) 


\section{Publications and invited presentations}

\section{FY13}

Formation, Stability and mobility of self-trapped excitations in Nal and $\mathrm{Nal}_{1-\mathrm{x}} \mathrm{TI}_{\mathrm{x}}$ from first principles

M.P. Prange, R.M. Van Ginhoven, N. Govind, F. Gao

Physical Review B 87 (11) 1151012013

Excited state electronic properties of sodium iodide and cesium iodide

L.W. Campbell, F. Gao

Journal of Luminescence 137 121-123 2013

Suppression of nonradiative recombination in ionic insulator defects: Role of fast electron trapping in Tl-doped CsI

J. Bang, Z. Wang, F. Gao, S. Meng, S. Zhang

Physical Review B 87 (20) 2052062013

Kinetic Monte Carlo simulations of excitation density dependent scintillation in CsI and CsI(TI)

Z. Wang, R.T. Williams, J.Q. Grim, F. Gao, S, Kerisit

Physica Status Solidi B 250 (8) 1532-1540 2013

Understanding fundamental mechanisms of nonlinearity in scintillators (invited presentation)

F. Gao - Hard X-Ray, Gamma-Ray, and Neutron Detector Physics XV - August 2013

Experimental and computational results on exciton/free-carrier ratio, hot/thermalized carrier diffusion, and linear/nonlinear rate constants affecting scintillator proportionality

R.T. Williams, J.Q. Grim, Q. Li, K.B. Ucer, G.A. Bizarri, S. Kerisit, F. Gao et al.

Proceedings of SPIE 8852 88520J-22 2013

Mechanisms of scintillator radiation response: Insights from Monte Carlo simulations (invited presentation)

S. Kerisit - International Conference on Advanced Scintillation Materials - September 2013

Microscopic mechanisms of electron-hole generation and their spatial distribution in inorganic scintillator (invited presentation)

F. Gao - International Conference on Advanced Scintillation Materials - September 2013 


\section{Publications and invited presentations}

\section{FY14}

Off-Center TI and Na Dopant Centers in CsI

R.M. Van Ginhoven and P.A. Schultz

Journal of Physics: Condensed Matter 25 (49) 4955042013

Monte Carlo simulation of gamma-ray response of $\mathrm{BaF}_{2}$ and $\mathrm{CaF}_{2}$

F. Gao, Y. Xie, Z. Wang, S. Kerisit, D. Wu, L.W. Campbell, R.M. Ginhoven, M.P. Prange

Journal of Applied Physics 114 (17) 1735122013

Kinetic Monte Carlo Simulations of Scintillation Processes in Nal(TI)

S. Kerisit, Z. Wang, R.T. Williams, J.Q. Grim, and F. Gao

IEEE Transactions on Nuclear Science 61(2) 860-869 2014

Science-driven search for new scintillator materials (invited presentation)

S. Kerisit - Scintillation Mechanisms in Gamma Detectors - April 2014

Radiation response of inorganic scintillators: Insights from Monte Carlo simulations (invited presentation)

S. Kerisit - Hard X-Ray, Gamma-Ray, and Neutron Detector Physics XVI - August 2014

Radiation response of inorganic scintillators: Insights from Monte Carlo simulations

M.P. Prange, D. Wu, L.W. Campbell, Y. Xie, F. Gao, S. Kerisit

Proceedings of SPIE 92132014

Calculation of energy and momentum relaxation rates of fast particles by phonons in crystals

M.P. Prange, L.W. Campbell

to be submitted by the end of FY14

Thermalization of energetic electrons and holes in halide scintillators

D. Wu, M.P. Prange, S. Kerisit

In preparation 


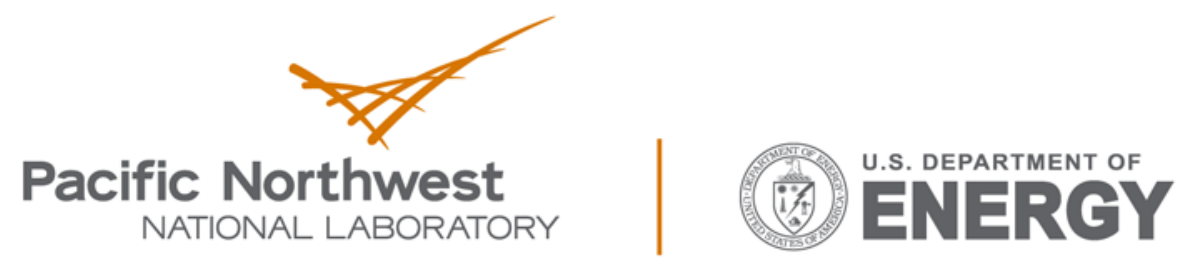

Proudly Operated by Battelle Since 1965

902 Battelle Boulevard

P.O. Box 999

Richland, WA 99352

1-888-375-PNNL (7665)

www.pnnl.gov 